\title{
COMPUTATIONAL FLUID DYNAMIC SIMULATION OF AIRFOILS IN UNSTEADY LOW REYNOLDS NUMBER FLOWS
}

\section{Mohammadreza Amiralaei}

BSc, Sharif University of Technology, Tehran, Iran, 2004 MSc, Sharif University of Technology, Tehran, Iran, 2007

A Dissertation

Presented to the School of Graduate Studies at

Ryerson University

in partial fulfillment of the

requirements for the degree of

Doctor of Philosophy

in the Program of Aerospace Engineering

Toronto, Ontario, Canada, 2012

(C)Mohammadreza Amiralaei, 2012 


\section{Author's Declaration}

I hereby declare that I am the sole author of this dissertation. This is a true copy of the dissertation, including any required final revisions, as accepted by my examiners.

I authorize Ryerson University to lend this dissertation to other institutions or individuals for the purpose of scholarly research.

I further authorize Ryerson University to reproduce this dissertation by photocopying or by other means, in total or in part, at the request of other institutions or individuals for the purpose of scholarly research.

I understand that my dissertation may be made electronically available to the public. 


\title{
Abstract Computational Fluid Dynamic Simulation of Airfoils in Unsteady Low Reynolds Number Flows
}

\author{
(C)Mohammadreza Amiralaei, 2012 \\ Doctor of Philosophy \\ Aerospace Engineering \\ Ryerson University
}

The inherent complexity of low Reynolds number (LRN) flows and their respective viscous vortical patterns demand an accurate solution method to achieve the desired accuracy. This complicated flow field needs even more robust methods when the flow is unsteady. The flow field of unsteady airfoils and wings in LRN regime is challenging to solve and Computational Fluid Dynamics (CFD) simulations stand out as solid solution techniques in this area. This thesis is motivated by an existing rotating-flapping mechanism, whose kinematics components can be broken into pitching, plunging and a novel figure-of-eight-like flapping motion of its blades and each blade's cross section. The focus is on two-dimensional low Reynolds number (LRN) flows using Computational Fluid Dynamics (CFD) and a Finite Volume Method (FVM). As one of the targets is to simulate a pair of blades, and consequently a pair of airfoils, a mesh motion library is developed to perform rotational and translational motions of multi-body configurations. The library and its sub-routines are tested on pairs of pitching, plunging and flapping airfoils, where the moving mesh problem is performed with a significant gain in the computational time compared to other moving mesh techniques such as Laplacian smoothing algorithm. The simulations of a single airfoil under harmonic and the novel figure-of-eight-like flapping motions, respectively, are conducted within $67 \%$ and $80 \%$ time it took to obtain a steady solution using the Laplace smoothing mesh motion algorithm, while the calculated force coefficients were in reasonably close agreement. Flow fields of single unsteady airfoils under pitching, plunging and figure-of-eight flapping motions are also simulated in this thesis accompanied with extensive parametric studies. The 
simulations of the considered figure-of-eight flapping pattern shows that its highly inclined asymmetrical kinematics results in higher vertical lift coefficients than the existing flapping patterns in the literature, useful for stable hovering flight.

The studies over paired-airfoils arrangements under pitching and plunging and the figureof-eight flapping motion show that the airfoil-airfoil interaction affects the fluid forces noticeably. The multi-plunging analysis, for example, reveals that the maximum lift coefficient is higher than that of a single plunging airfoil, while minimum drag coefficient is lower, showing the favorable effect of airfoil-airfoil interaction in the studied multi-plunging cases. 


\section{Acknowledgments}

I would like to extend my gratitude to my supervisors professors H. Alighanbari and S. M. Hashemi and thank them for their guidance, support and constant encouragement during the course of this research.

I would also like to thank Dr. D. Poirel of Royal Military College for serving as my external examiner. The positive feedback, suggestion and advice of my internal committee members professors B. Jubran, F. Sharifi, P. Walsh and J. Yokota from Ryerson University are greatly appreciated.

I would also thank my friends: Noushin, Robin and Masroor for their support, encouragement and advise, and the last but certainly not the least to my mother for her love and support and my father who always believed in me and encouraged me at all the times. 


\section{Contents}

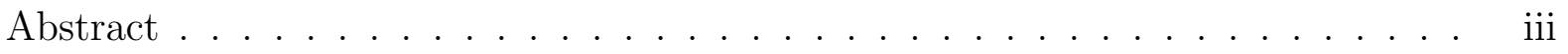

Acknowledgments . . . . . . . . . . . . . . . . v v

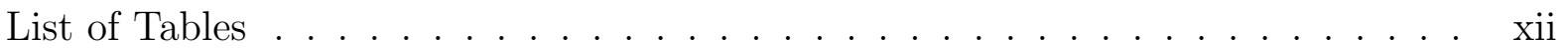

List of Figures . . . . . . . . . . . . . . . . . . xxiv

Nomenclature . . . . . . . . . . . . . . . . . . . . xxv

1 Introduction $\quad 1$

1.1 Overview . . . . . . . . . . . . . . . . . . . 1

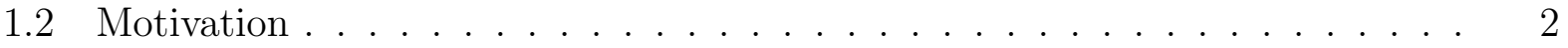

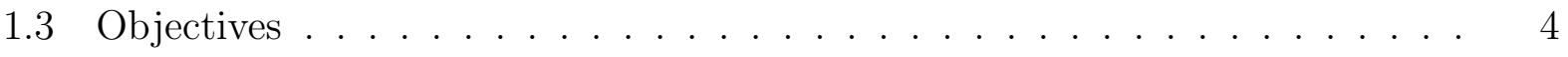

1.3.1 Modeling airfoils under different LRN unsteady conditions . . . . . 4

1.3.2 Extensive parametric studies . . . . . . . . . . . . . . . . . 4

1.3.3 Implementation, validation and modification of the fast and robust

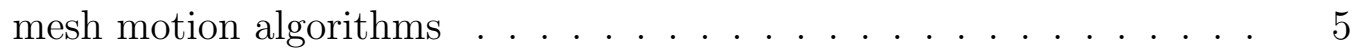

1.4 Thesis Contributions . . . . . . . . . . . . . . . 5

1.4.1 Analysis of flow field characteristics and force signatures of airfoils

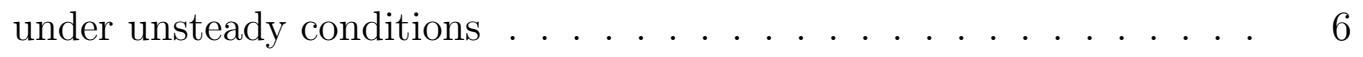

1.4.2 Development of mesh motion methodologies for simulating multi-body problems ...................... 7

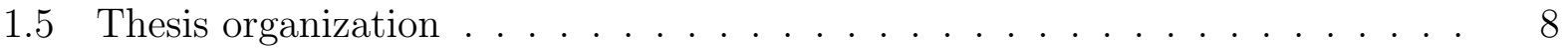

$\begin{array}{lll}2 & \text { Background and literature review } & 9\end{array}$ 


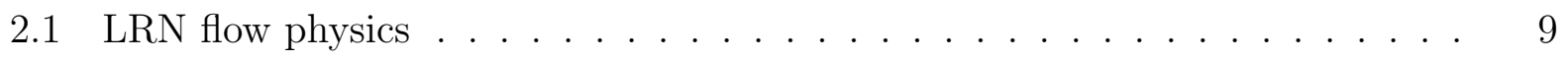

2.1.1 Dynamic stall and leading edge vortex . . . . . . . . . . . . 10

2.1.2 Rotational circulation (rotational forces) . . . . . . . . . . 10

2.1.3 Wake-capturing mechanism (wing-wake interaction) . . . . . . . 11

2.1.4 Weis-Fogh clap and fling mechanism . . . . . . . . . . . . 12

2.2 LRN solution methods . . . . . . . . . . . . . . . . . . . . . . 13

2.2 .1 Analytical methods . . . . . . . . . . . . . . . . . . . . 13

$2.2 .2 \quad$ Experimental methods . . . . . . . . . . . . . . . . . . . . . . 14

2.2 .3 Numerical methods . . . . . . . . . . . . . . . . . . . . . . . . . . 17

3 Numerical Simulation Method $\quad 23$

3.1 Introduction . . . . . . . . . . . . . . . . . . . . . . 23

3.2 Governing equations . . . . . . . . . . . . . . . . . 24

3.3 Discretization of N-S equations . . . . . . . . . . . . . . 27

3.3.1 Discretization of temporal and spatial terms in incompressible momen-

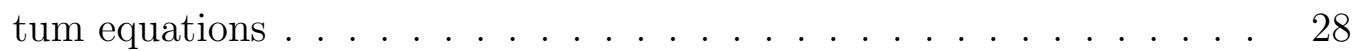

3.4 Boundary and initial conditions $\ldots \ldots \ldots \ldots \ldots \ldots \ldots$

3.5 Solution of the governing equations . . . . . . . . . . . . . . . 32

3.6 N-S equations for moving grid problems . . . . . . . . . . . . . . 34

3.7 Moving mesh algorithms . . . . . . . . . . . . . . . . 36

4 On the LRN flow characteristics of a pitching airfoil 43

4.1 Introduction . . . . . . . . . . . . . . . . . . . 44

4.2 Theory and numerical simulations . . . . . . . . . . . . . . 45

4.2 .1 Governing equations and flow solver . . . . . . . . . . . . 45

4.2 .2 Mesh generation and boundary conditions . . . . . . . . . . . 46

4.2 .3 Validation studies . . . . . . . . . . . . . . . . . . . . 47

4.3 Results and Discussion . . . . . . . . . . . . . . . . . 52 
4.3.1 Effects of amplitude of oscillation . . . . . . . . . . . 53

4.3 .2 Effects of reduced frequency . . . . . . . . . . . . . . 55

4.3 .3 Effects of Reynolds number _. . . . . . . . . . . . . . . . 59

4.4 Summary and conclusion . . . . . . . . . . . . . . . . . . 60

5 Numerical modeling of the flow field characteristics of a LRN plunging airfoil $\quad 63$

5.1 Introduction . . . . . . . . . . . . . . . . . . 64

5.2 Solution method . . . . . . . . . . . . . . . . . . 65

5.3 Reference velocity, force and moment coefficients calculations . . . . . . . . 66

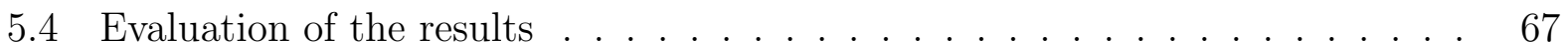

5.4 .1 Mesh and time step sensitivity analyses . . . . . . . . . . 67

5.4 .2 Validation studies . . . . . . . . . . . . . . . . . . 68

5.5 Evaluation of the results $\ldots \ldots \ldots \ldots \ldots$

5.5.1 Effect of the Strouhal number without freestream flow . . . . . . 71

5.5.2 Effect of the Strouhal number with freestream flow . . . . . . . 73

5.5 .3 Effect of the Reynolds number . . . . . . . . . . . . . . 77

5.6 Summary and conclusion . . . . . . . . . . . . . . . . . . . 82

6 Flow field characteristics of a figure-of-eight-like flapping airfoil $\quad 85$

6.1 Introduction . . . . . . . . . . . . . . . . . . 85

6.2 Numerical simulation method . . . . . . . . . . . . . . . . 88

6.3 Kinematics and equations of motion . . . . . . . . . . . . . 90

6.4 Reference velocity, force and moment coefficients calculations . . . . . . . . . 92

6.5 Evaluation of the results $\ldots \ldots \ldots . \ldots \ldots$

6.5.1 Effect of the amplitude of pitching oscillations . . . . . . . . . 97

6.5 .2 Effect of the phase angle . . . . . . . . . . . . . . . . 101

6.5.3 Effect of the mean angle of attack . . . . . . . . . . . 106 
6.5.4 Effect of the Reynolds number . . . . . . . . . . . . . . . . . 108

6.5.5 Effect of Strouhal Number . . . . . . . . . . . . . . . . . . . 109

6.5.6 Effect of the pitching axis location $\ldots \ldots \ldots \ldots$

6.6 Summary and conclusion . . . . . . . . . . . . . . . . . . . . . 114

$\begin{array}{lll}7 & \text { The unsteady aerodynamics of a pair of pitching airfoils } & 115\end{array}$

7.1 Introduction . . . . . . . . . . . . . . . . . 115

7.2 Numerical simulation method . . . . . . . . . . . . . . . . 116

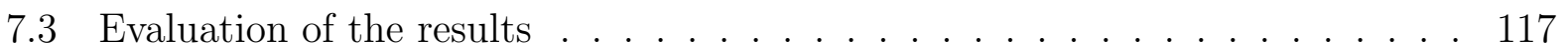

7.4 Results and discussion . . . . . . . . . . . . . . . . . . . . . 119

7.4.1 Effect of amplitude of pitching oscillations . . . . . . . . . 120

7.4 .2 Effect of Reynolds number . . . . . . . . . . . . . . . . 125

7.5 Summary and conclusion . . . . . . . . . . . . . . . . . 128

$\begin{array}{lll}8 & \text { On the force signatures of a pair of plunging airfoils } & 129\end{array}$

8.1 Introduction . . . . . . . . . . . . . . . . . . . . 130

8.2 Numerical simulation method . . . . . . . . . . . . . . . . . . 132

8.3 Evaluation of the numerical approach . . . . . . . . . . . . . 133

8.4 Results and discussion . . . . . . . . . . . . . . . . . 137

8.4.1 Effect of the Reynolds number . . . . . . . . . . . . . . . 137

8.4.2 Effect of the frequency of oscillations . . . . . . . . . . . . 142

8.4.3 Effect of the amplitude of oscillations . . . . . . . . . . . 146

8.4.4 Effect of the airfoil spacing . . . . . . . . . . . . . . . 151

8.5 Summary and conclusion . . . . . . . . . . . . . . . . . . 151

9 Multi figure-of-eight flapping airfoils $\quad 154$

9.1 Introduction . . . . . . . . . . . . . . . . . . . . . . . 154

9.2 Numerical simulation method . . . . . . . . . . . . . . . . 156

9.3 Evaluation of the results $\ldots \ldots \ldots \ldots \ldots$ 
9.4 Results and discussion . . . . . . . . . . . . . . . . . . . 159

9.5 Summary and conclusion . . . . . . . . . . . . . . . . 167

10 Concluding Remarks $\quad 169$

10.1 Future Work . . . . . . . . . . . . . . . . . . . . 170

10.2 Thesis Publications . . . . . . . . . . . . . . . . . . 171

10.2.1 Published Chapters in Books . . . . . . . . . . . . . . 172

10.2.2 Refereed Journal Publications . . . . . . . . . . . . . . . . 172

10.2.3 Submitted Journal Publications . . . . . . . . . . . . . . . . . 172

10.2.4 Refereed Conference Publications . . . . . . . . . . . . . . . 173

A Motion Methodology for Multi Figure-of-Eight Flapping Motions 176

$\begin{array}{ll}\text { Bibliography } & 210\end{array}$ 


\section{List of Tables}

Table 4.1 Spatial and temporal sensitivity analyses, difference is calculated based on the lift coefficient of the base line case $\left(26 \times 10^{3}\right.$ cells and 2000 time steps in a cycle). . . . . . . . . . . . . . . . . . . 49

Table 4.2 Summary of experimental and numerical pitching studies in the literature. 53

Table 5.1 Summary of experimental and numerical plunging studies in the literature. 71

Table 5.2 Mean lift and drag coefficients $\left(\bar{C}_{l}\right.$ and $\left.\bar{C}_{d}\right)$ for the investigated St where $\operatorname{Re}=100, Y_{0}=c$ and $U_{0} \neq 0 \ldots \ldots \ldots \ldots$

Table 5.3 Mean lift and drag coefficients $\left(\bar{C}_{l}\right.$ and $\left.\bar{C}_{d}\right)$ for the investigated Re when

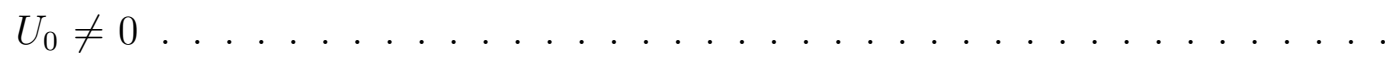

Table 6.1 Mean lift and drag coefficients $\left(\bar{C}_{l}\right.$ and $\left.\bar{C}_{d}\right)$ for the considered amplitudes of pitching oscillations $(\bar{\alpha}) \ldots \ldots \ldots \ldots \ldots$

Table 6.2 Mean lift and drag coefficients $\left(\bar{C}_{l}\right.$ and $\left.\bar{C}_{d}\right)$ for the considered phase angles $(\phi) \ldots \ldots \ldots \ldots \ldots \ldots \ldots$

Table 6.3 Mean lift and drag coefficients $\left(\bar{C}_{l}\right.$ and $\left.\bar{C}_{d}\right)$ for the considered Re. . . 110

Table 6.4 Mean lift and drag coefficients $\left(\bar{C}_{l}\right.$ and $\left.\bar{C}_{d}\right)$ for the considered St. . . . 112

Table 7.1 Mean lift and drag coefficients $\left(\bar{C}_{l}\right.$ and $\left.\bar{C}_{d}\right)$ for the considered amplitudes of pitching oscillations $(\theta)$ when $R e=50 \ldots \ldots \ldots \ldots$

Table 7.2 Mean lift and drag coefficients $\left(\bar{C}_{l}\right.$ and $\left.\bar{C}_{d}\right)$ for the considered amplitudes of pitching oscillations $(\theta)$ when $\mathrm{Re}=75 \ldots \ldots \ldots \ldots$. . . . . . 123 
Table 7.3 Mean lift and drag coefficients $\left(\bar{C}_{l}\right.$ and $\left.\bar{C}_{d}\right)$ for the considered amplitudes of pitching oscillations $(\theta)$ when $\operatorname{Re}=100 \ldots \ldots$. . . . . . . . . .

Table 7.4 Mean lift and drag coefficients $\left(\bar{C}_{l}\right.$ and $\left.\bar{C}_{d}\right)$ for the considered Re when $\theta=30^{\circ} \ldots \ldots \ldots \ldots \ldots \ldots \ldots \ldots$

Table 7.5 Mean lift and drag coefficients $\left(\bar{C}_{l}\right.$ and $\left.\bar{C}_{d}\right)$ for the considered Re when $\theta=45^{\circ} \ldots \ldots \ldots \ldots \ldots \ldots \ldots \ldots$

Table 8.1 Parameter settings for the conducted case-studies . . . . . . . . . . 137

Table 8.2 Mean lift and drag coefficients $\left(\bar{C}_{l}\right.$ and $\left.\bar{C}_{d}\right)$ for the considered Re. . . 142

Table 8.3 Mean lift and drag coefficients $\left(\bar{C}_{l}\right.$ and $\left.\bar{C}_{d}\right)$ for the considered frequencies of oscillations $(f) \ldots \ldots \ldots \ldots \ldots \ldots$

Table 8.4 Mean lift and drag coefficients $\left(\bar{C}_{l}\right.$ and $\left.\bar{C}_{d}\right)$ for the considered amplitude of oscillations $\left(Y_{0}\right)$ when $g=1.5 c \ldots \ldots \ldots$. . . . . . . . . 149

Table 8.5 Mean lift and drag coefficients $\left(\bar{C}_{l}\right.$ and $\left.\bar{C}_{d}\right)$ for the considered amplitude of oscillations $\left(Y_{0}\right)$ when $g=1.5 c \ldots \ldots \ldots$. . . . . . . . 150 


\section{List of Figures}

Figure 1.1 The rotating-flapping mechanism, adopted from Ania [5]. . . . . . . . 2

Figure 2.1 LEV and TEV around a flapping airfoil resulted from the present investigations. . . . . . . . . . . . . . . . . .

Figure 2.2 Wake-capturing mechanism during the upstroke motion of a plunging airfoil; (a) airfoil approaches the vortex; (b) airfoil encounters the vortex, corresponding to the simulations of Chapter $5 \ldots \ldots \ldots . . . . .$.

Figure 3.1 Arbitrary polyhedral cell around point $\mathrm{P}$ in the computational domain, adopted from Jasak and Tuković [107]. . . . . . . . . . . . . . . . . . 35

Figure 4.1 Schematic of the O-type grid around the airfoil. . . . . . . . . . . 46

Figure 4.2 Schematic of the O-type grid around the airfoil. . . . . . . . . . . . . 47

Figure 4.3 Instantaneous peak lift coefficients $\left(C_{l}\right)$ versus nondimensional time $(\tau)$ for different mesh sizes $(k=0.1, x / c=0.25):+, 5 \times 10^{3} ; \diamond, 11 \times 10^{3} ; \square$, $26 \times 10^{3} ; \times, 50 \times 10^{3}$, (a) $d=2^{\circ}$ and $\operatorname{Re}=555 ;(\mathrm{b}): d=10^{\circ}$ and $\operatorname{Re}=5000 . \quad 48$

Figure 4.4 Instantaneous lift coefficient of a pitching cylinder at $\operatorname{Re}=40, U_{w}=$ $0.2 \sin (0.1 \pi t):+$, Mahfouz and Badr [133]; ×, Okajima et al. [134]; *, present

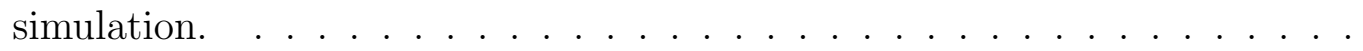

Figure 4.5 Normal force coefficient $\left(C_{n}\right)$ of a pitching NACA0012 at Re $=3000$ $\left(\alpha=15^{\circ}+10^{\circ} \cos (2 \pi f t), k=0.25, x / c=0.25\right):-$, present simulation; o, Akbari and Price [68]. . . . . . . . . . . . . . . . 
Figure 4.6 Instantaneous lift coefficient versus nondimensional time $(\mathrm{Re}=555$, $\left.k=0.1, d=2^{\circ}\right)$ :, viscous NS simulation; $\times$, inviscid N-S simulation; *, Theodorsen.

Figure 4.7 Instantaneous lift coefficient versus nondimensional time $(\operatorname{Re}=555$, $k=0.1, d=10^{\circ}$ ): o, numerical; -, Theodorsen; (Airfoil pitch angle is also shown on the figure as $\alpha / 2)$.

Figure 4.8 Schematic view of the conducted case studies. . . . . . . . . . . 53

Figure 4.9 Instantaneous lift coefficient $\left(C_{l}\right)$ versus nondimensional time $(\tau):(-)$,

$$
d=2^{\circ} ;(+), d=4^{\circ} ;(\diamond), d=6^{\circ} ;(\square), d=8^{\circ} ;(\times), d=10^{\circ} .
$$

Figure 4.10 The generated vortical structures after the peak lift coefficient $C_{l_{\text {max }}}$,

$$
d=10^{\circ}, k=0.1, \operatorname{Re}=555:(\mathrm{a}), \alpha=-9.75^{\circ} ;(\mathrm{b}), \alpha=-7.8^{\circ} ;(\mathrm{c}), \alpha=-5.6^{\circ},
$$

the airfoil is in up-stroke.

Figure 4.11 Lift coefficient $\left(C_{l}\right)$ versus angle of attack $(\alpha), k=0.1, \operatorname{Re}=555:(-)$,

$$
d=2^{\circ} ;(+), d=4^{\circ} ;(\diamond), d=6^{\circ} ;(\square), d=8^{\circ} ;(\times), d=10^{\circ} . \ldots
$$

Figure 4.12 Drag coefficient $\left(C_{d}\right)$ versus angle of attack $(\alpha), k=0.1, \operatorname{Re}=555$ :

$$
(-), d=2^{\circ} ;(+), d=4^{\circ} ;(\diamond), d=6^{\circ} ;(\square), d=8^{\circ} ;(\times), d=10^{\circ} .
$$

Figure 4.13 Lift coefficient $\left(C_{l}\right)$ versus angle of attack $\left.(\alpha), d=4^{\circ}, \operatorname{Re}=555\right)$ : (-),

$$
k=0.1 ;(+), k=0.125 ;(\diamond), k=0.17 ;(\times), k=0.25 .
$$

Figure 4.14 Lift coefficient $\left(C_{l}\right)$ versus angle of attack $(\alpha), d=6^{\circ}, \operatorname{Re}=555:(-)$,

$$
k=0.1 ;(+), k=0.125 ;(\diamond), k=0.17 ;(\times), k=0.25 \ldots \ldots \ldots
$$

Figure 4.15 Lift coefficient $\left(C_{l}\right)$ versus angle of attack $(\alpha), d=10^{\circ}, \operatorname{Re}=555$ : (-),

$$
k=0.1 ;(+), k=0.125 ;(\diamond), k=0.17 ;(\times), k=0.25 \ldots \ldots \ldots
$$

Figure 4.16 Drag coefficient $\left(C_{d}\right)$ versus angle of attack $(\alpha), d=10^{\circ}, \operatorname{Re}=555$ :

$$
(-), k=0.1 ;(+), k=0.125 ;(\diamond), k=0.17 ;(\times), k=0.25 \ldots \ldots \ldots
$$

Figure 4.17 Lift coefficient $\left(C_{l}\right)$ versus angle of attack $(\alpha), d=10^{\circ}, k=0.1$ : $(-)$,

$$
\operatorname{Re}=555 ;(+), \operatorname{Re}=1000 ;(\diamond), \operatorname{Re}=2000 ;(\times), \operatorname{Re}=5000 . \ldots \ldots
$$


Figure 4.18 Vortical patterns around the airfoil when maximum lift coefficient $\left(C_{l_{\max }}\right)$ is obtained $\left(d=10^{\circ}, k=0.1\right) \ldots \ldots \ldots$

Figure 4.19 Lift coefficient $\left(C_{l}\right)$ versus angle of attack $(\alpha), d=2^{\circ}, k=0.1:(-)$, $\operatorname{Re}$ $=555 ;(+), \operatorname{Re}=1000 ;(\diamond), \operatorname{Re}=2000 ;(\times), \operatorname{Re}=5000 \ldots \ldots 1$

Figure 4.20 Drag coefficient $\left(C_{d}\right)$ versus angle of attack $(\alpha), d=10^{\circ}, k=0.1:(-)$, $\operatorname{Re}=555 ;(+), \operatorname{Re}=1000 ;(\diamond), \operatorname{Re}=2000 ;(\times), \operatorname{Re}=5000 . \ldots \ldots 1$

Figure 5.1 Schematic of the airfoil and the computational domain. . . . . . . 65

Figure 5.2 Schematic of the plunging airfoil and force and moment signatures. $\quad$ • $\quad 67$

Figure 5.3 Instantaneous lift coefficient $\left(C_{l}\right)$ versus nondimensional time $(\tau)$ for St $=0.16, \operatorname{Re}=100$, and $U_{0}=0,(\mathrm{a}):(-) 24 \times 10^{3},(+) 48 \times 10^{3},(\times) 96 \times 10^{3}$ cells with 2000 time steps;(b): (-) 1000, (+) 2000, (×) 4000 time steps with $48 \times 10^{3}$ cells. . . . . . . . . . . . . . . . . . . . . 68

Figure 5.4 Instantaneous drag coefficient $\left(C_{d}\right)$ versus time $(t)$ for a static cylinder

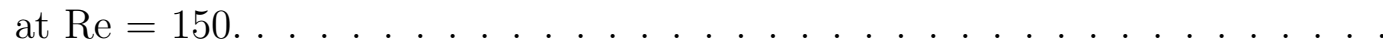

Figure 5.5 Instantaneous lift and drag coefficients $\left(C_{l}\right.$ and $\left.C_{d}\right)$ versus time $(t)$ of a plunging cylinder following Equation 5.4 at $\mathrm{Re}=185, A_{e}=0.2 c$ ( $c$ being the cylinder diameter) and $f_{e}=0.154 \ldots \ldots \ldots \ldots$

Figure 5.6 Instantaneous lift coefficient (left) and drag coefficient (right) versus $\tau$ for a plunging NACA0012 airfoil with $Y_{0} / c=0.0125$ and St $=0.03:(*)$, Lian and Shyy [138]; $(+)$, Young [139]; (×), present simulations. . . . . . . . . 70

Figure 5.7 Instantaneous lift coefficient $\left(C_{l}\right)$ versus nondimensional time $(\tau)$ when $\mathrm{Re}=100$ and $U_{0}=0:(-) \mathrm{St}=0.32,(+) \mathrm{St}=0.16,(\times) \mathrm{St}=0.11 . \ldots$

Figure 5.8 Pressure contours when maximum lift coefficient $\left(C_{l_{\max }}\right)$ is achieved at $\mathrm{Re}=100$ and $U_{0}=0:$ (a) St $=0.32$, (b) St $=0.16$, (c) St $=0.11 \ldots \ldots$

Figure 5.9 Instantaneous lift coefficient $\left(C_{l}\right)$ versus nondimensional time $(\tau)$ for

$$
\operatorname{Re}=100, Y_{0}=c \text { and } U_{0} \neq 0:(-) \text { St }=0.08,(+) \mathrm{St}=0.16,(\times) \text { St }=0.32 .
$$


Figure 5.10 Instantaneous drag coefficient $\left(C_{d}\right)$ versus nondimensional time $(\tau)$ for

$$
\mathrm{Re}=100, Y_{0}=c \text { and } U_{0} \neq 0:(-) \mathrm{St}=0.08,(+) \mathrm{St}=0.16,(\times) \mathrm{St}=0.32 .
$$

Figure 5.11 Vortical patterns around the airfoil when first maximum drag coefficient $\left(C_{d_{\min }}\right)$ is obtained for $Y_{0}=c, \operatorname{Re}=100$ and $U_{0} \neq 0$ : (a), St $=0.08$; (b), St $=0.16 ;(\mathrm{c}), \mathrm{St}=0.32$.

Figure 5.12 Instantaneous pitching moment coefficient $\left(C_{m}\right)$ versus nondimensional time $(\tau)$ for $\operatorname{Re}=100, Y_{0}=c$ and $U_{0} \neq 0:(-)$ St $=0.08,(+)$ St $=0.16,(\times)$ $\mathrm{St}=0.32$.

Figure 5.13 Instantaneous lift coefficient $\left(C_{l}\right)$ versus nondimensional $(\tau)$ when St $=0.16$ and $U_{0}=0:(-), \operatorname{Re}=50 ;(+), \operatorname{Re}=100$.

Figure 5.14 Instantaneous lift coefficient $\left(C_{l}\right)$ versus nondimensional time $(\tau)$ for St $=0.16$ when $U_{0} \neq 0:(-) \operatorname{Re}=100,(+) \operatorname{Re}=200,(\times) \operatorname{Re}=500 \ldots \ldots$ 78

Figure 5.15 Vortical patterns around the airfoil when maximum lift coefficient $\left(C_{l_{\max }}\right)$ is obtained for $\mathrm{St}=0.16$ and $U_{0} \neq 0$ : (a), Re $=100 ;(\mathrm{b}), \operatorname{Re}=$ $200 ;(\mathrm{c}), \operatorname{Re}=500 \ldots \ldots \ldots \ldots \ldots$

Figure 5.16 Instantaneous drag coefficient $\left(C_{d}\right)$ versus nondimensional time $(\tau)$ for St $=0.16$ when $U_{0} \neq 0:(-) \operatorname{Re}=100,(+) \operatorname{Re}=200,(\times) \operatorname{Re}=500 \ldots \ldots$

Figure 5.17 Vortical patterns around the airfoil when maximum drag coefficient $\left(C_{d_{\max }}\right)$ at $\tau=0.52$ is obtained for $\mathrm{St}=0.16$ and $U_{0} \neq 0$ : (a), Re $=100 ;(\mathrm{b})$, $\operatorname{Re}=200 ;(\mathrm{c}), \operatorname{Re}=500$.

Figure 5.18 Instantaneous pitching moment coefficient $\left(C_{m}\right)$ versus nondimensional time $(\tau)$ for St $=0.16$ when $U_{0} \neq 0:(-) \operatorname{Re}=100,(+) \operatorname{Re}=200,(\times) \operatorname{Re}=$ 500.

Figure 5.19 Vortical patterns around the airfoil when minimum moment coefficient $\left(C_{m_{\min }}\right)$ at $\tau=0.6$ is obtained for $\mathrm{St}=0.16$ and $U_{0} \neq 0:(\mathrm{a}), \operatorname{Re}=100 ;(\mathrm{b})$, $\operatorname{Re}=200 ;(\mathrm{c}), \operatorname{Re}=500$, the vertical position is the same for all figures. . .

Figure 6.1 Schematic of the airfoil and the computational domain. . . . . . . . . 
Figure 6.2 Schematic of the O-type grid around the airfoil. . . . . . . . . . . . 89

Figure 6.3 The schematic of the pair of rotating-flapping blade configuration. . . 90

Figure 6.4 The 2-D kinematic pattern obtained from Equations 6.2. . . . . . . . 92

Figure 6.5 Forces and moment on the airfoil. . . . . . . . . . . . . . . . . . 93

Figure 6.6 Instantaneous lift coefficient $\left(C_{l}\right)$ versus nondimensional time $(\tau)$ for

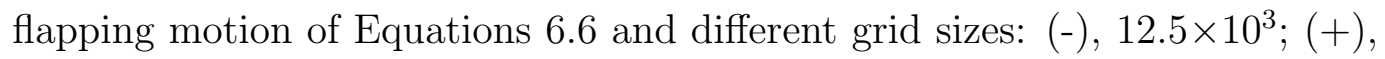
$25 \times 10^{3} ; \times, 50 \times 10^{3} ;($ o $), 100 \times 10^{3}$.

Figure 6.7 Instantaneous lift coefficient $\left(C_{l}\right)$ versus nondimensional time $(\tau)$ for flapping motion of Equations 6.6 and different time steps: $(-), 500 ;(+), 1000$; $(\times), 2000 \ldots \ldots \ldots \ldots \ldots \ldots \ldots$

Figure 6.8 Instantaneous lift coefficient $\left(C_{l}\right)$ versus nondimensional time $(\tau)$ of for the flapping motion of Equations 6.6: (-), present simulation; (+), Bos et al. [88]; $(\times)$, Wang et al. [58]

Figure 6.9 Vortical patterns around the airfoil for $R=1.4 c, d=0.7 c, f=0.25$,

$$
\begin{aligned}
& \alpha_{0}=90^{\circ}, \phi=0, \operatorname{Re}=75, \bar{\alpha}=45^{\circ}: \text { (a) } \tau=0.04 \text {; (b) } \tau=0.1 ; \text { (c) } \tau=0.16 ; \\
& \text { (d) } \tau=0.24 ; \text { (e) } \tau=0.4 ; \text { (f) } \tau=0.54 ; \text { (g) } \tau=0.72 \text {; (h) } \tau=0.86 \text {; (i) } \tau=0.94 .98
\end{aligned}
$$

Figure 6.10 Locations of the airfoil on the investigated flapping pattern corresponding to Fig 6.9. . . . . . . . . . . . . . . . . . .

Figure 6.11 Instantaneous lift coefficient $\left(C_{l}\right)$ versus nondimensional time $(\tau)$ for

$$
\begin{aligned}
& R=1.4 c, d=0.7 c, f=0.25, \alpha_{0}=90^{\circ}, \phi=0, \operatorname{Re}=75:(-), \bar{\alpha}=15^{\circ} ;(+), \bar{\alpha} \\
& =30^{\circ} ;(\times), \bar{\alpha}=45^{\circ} ;(\circ), \bar{\alpha}=60^{\circ} \text {. }
\end{aligned}
$$

Figure 6.12 LEV and TEV around the ellipse when the first major maximum lift coefficient $\left(C_{l_{\max }}\right)$ is obtained: (a) $\bar{\alpha}=15^{\circ}$; (b) $\bar{\alpha}=30^{\circ}$; (c) $\bar{\alpha}=45^{\circ}$; (d) $\bar{\alpha}$ $=60^{\circ}$.

Figure 6.13 LEV and TEV around the ellipse when the second major maximum lift coefficient $\left(C_{l_{\max }}\right)$ is obtained: (a) $\bar{\alpha}=15^{\circ}$; (b) $\bar{\alpha}=30^{\circ} ;$ (c) $\bar{\alpha}=45^{\circ}$; (d) $\bar{\alpha}=60^{\circ}$. 
Figure 6.14 Instantaneous lift coefficient $\left(C_{l}\right)$ versus nondimensional time $(\tau)$ for

$$
\begin{aligned}
& R=1.4 c, d=0.7 c, f=0.25, \alpha_{0}=90^{\circ}, \phi=0, \operatorname{Re}=75:(-), \bar{\alpha}=15^{\circ} ;(+), \bar{\alpha} \\
& =30^{\circ} ;(\times), \bar{\alpha}=45^{\circ} ;(\circ), \bar{\alpha}=60^{\circ} \ldots \ldots \ldots \ldots
\end{aligned}
$$

Figure 6.15 Instantaneous pitching moment coefficient $\left(C_{m}\right)$ versus nondimensional time $(\tau)$ for $R=1.4 c, d=0.7 c, f=0.25, \alpha_{0}=90^{\circ}, \phi=0, \operatorname{Re}=75:(-), \bar{\alpha}$ $=15^{\circ} ;(+), \bar{\alpha}=30^{\circ} ;(\times), \bar{\alpha}=45^{\circ} ;(\circ), \bar{\alpha}=60^{\circ}$.

Figure 6.16 Instantaneous lift coefficient $\left(C_{l}\right)$ versus nondimensional time $(\tau)$ for $R=1.4 c, d=0.7 c, f=0.25, \alpha_{0}=90^{\circ}, \bar{\alpha}=45^{\circ}$ and $\operatorname{Re}=75:(-), \phi=-30^{\circ} ;$ $(+), \phi=0 ;(\times), \phi=30^{\circ}$.

Figure 6.17 Vorticity contours at the first maximum lift coefficient $\left(C_{l_{\max }}\right)$ for $R=$ $1.4 c, d=0.7 c, f=0.25, \alpha_{0}=90^{\circ}, \bar{\alpha}=45^{\circ}$ and $\operatorname{Re}=75:$ (a) $\phi=-30^{\circ} ;$ (b) $\phi$ $=0 ;(\mathrm{c}) \phi=30^{\circ}$.

Figure 6.18 Vorticity contours at the second maximum lift coefficient $\left(C_{l_{\max }}\right)$ for $R=1.4 c, d=0.7 c, f=0.25, \alpha_{0}=90^{\circ}, \bar{\alpha}=45^{\circ}$ and $\operatorname{Re}=75:$ (a) $\phi=-30^{\circ} ;$ (b) $\phi=0 ;$ (c) $\phi=30^{\circ}$. 104

Figure 6.19 Instantaneous drag coefficient $\left(C_{d}\right)$ versus nondimensional time $(\tau)$ for $R=1.4 c, d=0.7 c, f=0.25, \alpha_{0}=90^{\circ}, \bar{\alpha}=45^{\circ}$ and $\operatorname{Re}=75:(-), \phi=-30^{\circ} ;$ $(+), \phi=0 ;(\times), \phi=30^{\circ}$.

Figure 6.20 Instantaneous pitching moment coefficient $\left(C_{m}\right)$ versus nondimensional time $(\tau)$ for $R=1.4 c, d=0.7 c, f=0.25, \alpha_{0}=90^{\circ}, \bar{\alpha}=45^{\circ}$ and $\operatorname{Re}=75$ : $(-), \phi=-30^{\circ} ;(+), \phi=0 ;(\times), \phi=30^{\circ} \ldots \ldots \ldots$ 106

Figure 6.21 Instantaneous lift coefficient $\left(C_{l}\right)$ versus nondimensional time $(\tau)$ for $R=1.4 c, d=0.7 c, f=0.25, \bar{\alpha}=45^{\circ}, \phi=0$ and $\operatorname{Re}=75:(-), \alpha_{0}=45^{\circ} ;$ $(+), \alpha_{0}=60^{\circ} ;(\times), \alpha_{0}=90^{\circ} \ldots \ldots \ldots \ldots \ldots$

Figure 6.22 Vorticity contours at the first maximum lift coefficient $\left(C_{l_{\max }}\right)$ for $R=$ $1.4 c, d=0.7 c, f=0.25, \bar{\alpha}=45^{\circ}, \phi=0$ and $\operatorname{Re}=75:$ (a) $\alpha_{0}=45^{\circ}$; (b) $\alpha_{0}$

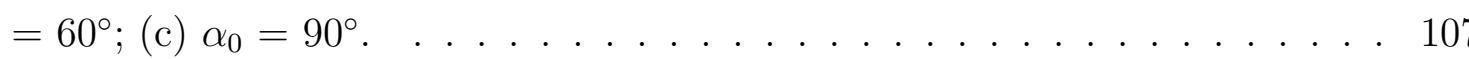


Figure 6.23 Vorticity contours at the second maximum lift coefficient $\left(C_{l_{\max }}\right)$ for

$$
\begin{aligned}
& R=1.4 c, d=0.7 c, f=0.25, \bar{\alpha}=45^{\circ}, \phi=0 \text { and } \operatorname{Re}=75: \text { (a) } \alpha_{0}=45^{\circ} ;(\mathrm{b}) \\
& \alpha_{0}=60^{\circ} ; \text { (c) } \alpha_{0}=90^{\circ} \text {. }
\end{aligned}
$$

Figure 6.24 Instantaneous lift coefficient $\left(C_{l}\right)$ versus nondimensional time $(\tau)$ for

$$
\begin{aligned}
& R=1.4 c, d=0.7 c, f=0.25, \alpha_{0}=90^{\circ}, \bar{\alpha}=45^{\circ}, \phi=0:(-), \operatorname{Re}=37.5 ;(+), \\
& \operatorname{Re}=75 ;(\times), \operatorname{Re}=150 \ldots \ldots \ldots \ldots \ldots
\end{aligned}
$$

Figure 6.25 Vorticity contours for $R=1.4 c, d=0.7 c, f=0.25, \alpha_{0}=90^{\circ}, \bar{\alpha}=$ $45^{\circ}, \phi=0$ : (a) first $C_{l_{\max }}$ at $\operatorname{Re}=37.5$; (b) first $C_{l_{\max }}$ at $\operatorname{Re}=75$; (c) second $C_{l_{\max }}$ at $\operatorname{Re}=37.5 ;(\mathrm{d})$ second $C_{l_{\max }}$ at $\mathrm{Re}=75$.

Figure 6.26 Instantaneous pitching moment coefficient $\left(C_{m}\right)$ versus nondimensional time $(\tau)$ for $R=1.4 c, d=0.7 c, f=0.25, \alpha_{0}=90^{\circ}, \bar{\alpha}=45^{\circ}, \phi=0:(-)$, Re $=37.5 ;(+), \operatorname{Re}=75 ;(\times), \operatorname{Re}=150 \ldots \ldots \ldots \ldots$

Figure 6.27 Instantaneous lift coefficient $\left(C_{l}\right)$ versus nondimensional time $(\tau)$ for $R=1.4 c, f=0.25, \alpha_{0}=90^{\circ}, \bar{\alpha}=45^{\circ}, \phi=0$ and $\operatorname{Re}=75:(-)$, St $=0.116 ;$ $(+)$, St $=0.113 ;(\times)$, St $=0.110$.

Figure 6.28 Instantaneous drag coefficient $\left(C_{d}\right)$ versus nondimensional time $(\tau)$ for

$$
\begin{aligned}
& R=1.4 c, f=0.25, \alpha_{0}=90^{\circ}, \bar{\alpha}=45^{\circ}, \phi=0 \text { and } \operatorname{Re}=75:(-), \text { St }=0.116 ; \\
& (+), \mathrm{St}=0.113 ;(\times), \mathrm{St}=0.110 \text {. }
\end{aligned}
$$

Figure 6.29 Instantaneous lift coefficient $\left(C_{l}\right)$ versus nondimensional time $(\tau)$ for $d=0.7 c, f=0.25, \alpha_{0}=90^{\circ}, \bar{\alpha}=45^{\circ}, \phi=0$ and $\operatorname{Re}=75:(-)$, St $=0.153 ;$ $(+), \mathrm{St}=0.113 \ldots \ldots \ldots \ldots \ldots \ldots \ldots \ldots$

Figure 6.30 Instantaneous lift coefficient $\left(C_{l}\right)$ versus nondimensional time $(\tau)$ for $R=1.4 c, d=0.7 c, f=0.25, \alpha_{0}=90^{\circ}, \bar{\alpha}=45^{\circ}, \phi=0$ and $\operatorname{Re}=75:(-), x / c$ $=0.25 ;(+), x / c=0.5 \ldots \ldots \ldots \ldots \ldots$

Figure 6.31 Instantaneous pitching moment coefficient $\left(C_{m}\right)$ versus nondimensional time $(\tau)$ for $R=1.4 c, d=0.7 c, f=0.25, \alpha_{0}=90^{\circ}, \bar{\alpha}=45^{\circ}, \phi=0$ and $\operatorname{Re}$ $=75:(-), x / c=0.25 ;(+), x / c=0.5$. 
Figure 7.1 Schematic of the computational domain and the pitching airfoils. . . . 117

Figure 7.2 Instantaneous lift coefficient $\left(C_{l}\right)$ versus nondimensional time $(\tau)$ for

$$
\operatorname{Re}=50 \text { and } \theta=15^{\circ}:(+), 23 \times 10^{3} ;(\mathrm{o}), 47 \times 10^{3},(\times), 94 \times 10^{3} .
$$

Figure 7.3 Instantaneous drag coefficient $\left(C_{d}\right)$ versus nondimensional time $(\tau)$ for Re $=3000$ following Equation 7.2, (+): present simulation, $(\times)$ : Akbari and Price $[68] \ldots \ldots \ldots \ldots \ldots \ldots \ldots \ldots \ldots$

Figure 7.4 Instantaneous lift and drag coefficients $\left(C_{l}\right.$ and $\left.C_{d}\right)$ versus nondimensional time $(\tau)$ for $\operatorname{Re}=100$ and $\theta=15^{\circ},(+$ and $\times)$, left airfoil; $(\mathrm{o}$ and $\diamond)$, right airfoil.

Figure 7.5 Instantaneous lift and drag coefficients $\left(C_{l}\right.$ and $\left.C_{d}\right)$ versus nondimensional time $(\tau)$ for $\operatorname{Re}=100$ and $\theta=30^{\circ},(+$ and $\times)$, left airfoil; (o and $\left.\diamond\right)$, right airfoil.

Figure 7.6 Instantaneous lift and drag coefficients $\left(C_{l}\right.$ and $\left.C_{d}\right)$ versus nondimensional time $(\tau)$ for $\operatorname{Re}=100$ and $\theta=45^{\circ},(+$ and $\times)$, left airfoil; (o and $\left.\diamond\right)$, right airfoil.

Figure 7.7 Instantaneous lift coefficient $\left(C_{l}\right)$ versus nondimensional time $(\tau)$ for right airfoil, $\operatorname{Re}=50,(+), \theta=15^{\circ} ;(\mathrm{o}), \theta=30^{\circ} ;(\times), \theta=45^{\circ}$.

Figure 7.8 Instantaneous lift coefficient $\left(C_{l}\right)$ versus nondimensional time $(\tau)$ for right airfoil, $\operatorname{Re}=75:(+), \theta=15^{\circ} ;(\mathrm{o}), \theta=30^{\circ} ;(\times), \theta=45^{\circ}$.

Figure 7.9 Instantaneous drag coefficient $\left(C_{d}\right)$ versus nondimensional time $(\tau)$ for right airfoil, $\operatorname{Re}=75:(+), \theta=15^{\circ} ;(\mathrm{o}), \theta=30^{\circ} ;(\times), \theta=45^{\circ}$.

Figure 7.10 Instantaneous lift coefficient $\left(C_{l}\right)$ versus nondimensional time $(\tau)$ for right airfoil, $\operatorname{Re}=100:(+), \theta=15^{\circ} ;(\mathrm{o}), \theta=30^{\circ} ;(\times), \theta=45^{\circ}$.

Figure 7.11 Instantaneous drag coefficient $\left(C_{d}\right)$ versus nondimensional time $(\tau)$ for right airfoil, $\operatorname{Re}=100:(+), \theta=15^{\circ} ;(\mathrm{o}), \theta=30^{\circ} ;(\times), \theta=45^{\circ}$.

Figure 7.12 Instantaneous pitching moment coefficient $\left(C_{m}\right)$ versus nondimensional time $(\tau)$ for right airfoil, $\operatorname{Re}=100:(+), \theta=15^{\circ} ;(\mathrm{o}), \theta=30^{\circ} ;(\times), \theta=45^{\circ}$. 
Figure 7.13 Instantaneous lift coefficient $\left(C_{l}\right)$ versus nondimensional time $(\tau)$ for right airfoil when $\theta=30^{\circ}:(+), \operatorname{Re}=50 ;(\mathrm{o}), \operatorname{Re}=75 ;(\times), \operatorname{Re}=100 . \quad \ldots 126$

Figure 7.14 Instantaneous drag coefficient $\left(C_{d}\right)$ versus nondimensional time $(\tau)$ for right airfoil when $\theta=30^{\circ}:(+), \operatorname{Re}=50 ;($ o), $\operatorname{Re}=75 ;(\times), \operatorname{Re}=100 . \quad$. 126

Figure 7.15 Instantaneous lift coefficient $\left(C_{l}\right)$ versus nondimensional time $(\tau)$ for right airfoil when $\theta=45^{\circ}:(+), \operatorname{Re}=50 ;(\mathrm{o}), \operatorname{Re}=75 ;(\times), \operatorname{Re}=100$. . .

Figure 7.16 Instantaneous drag coefficient $\left(C_{d}\right)$ versus nondimensional time $(\tau)$ for right airfoil when $\theta=45^{\circ}:(+), \operatorname{Re}=50 ;(\mathrm{o}), \operatorname{Re}=75 ;(\times), \operatorname{Re}=100$. . .

Figure 8.1 Schematic of the plunging airfoils and the computational domain. . . 132

Figure 8.2 Instantaneous lift coefficient $\left(C_{l}\right)$ versus nondimensional time $(\tau)$ for $g=1.5, f=0.25, Y_{0}=0.5$ and $\operatorname{Re}=75,(\mathrm{a}):(*) 25 \times 10^{3},(+) 50 \times 10^{3},(\times)$ $75 \times 10^{3}$ cell; (b): (*) 1000, (+) 2000, (× 4000 time steps within a cycle. . . 134

Figure 8.3 Instantaneous lift coefficient $\left(C_{l}\right)$ versus nondimensional time $(\tau)$ for a $2 \%$ thickness ellipsoidal airfoil plunging with $y(t)=Y_{0} \sin (2 \pi f t)$ with $Y_{0}=$ $0.5 c$ and $f=1 ;(-)$, dynamic layering; $(+)$, Laplace smoothing. . . . . . . . . 135

Figure 8.4 Schematic of cylinders and the computational domain. . . . . . . . 136

Figure 8.5 Vortical pattern in the downstream wake of the side-by-side cylinders plunging according to Equations 8.3 at $\tau \approx 0.5 \ldots \ldots \ldots$. . . . . . . . . 136

Figure 8.6 Instantaneous lift and drag coefficients $\left(C_{l}\right.$ and $\left.C_{d}\right)$ versus time for a pair of cylinders plunging according to Equations 8.3 with $g_{0}=1.8, f=$ $0.154, D=1$, and $A_{e}=0.2 ;(-)$, upper cylinder; (..), lower cylinder. . . . . 136

Figure 8.7 Instantaneous lift coefficient $\left(C_{l}\right)$ versus nondimensional time $(\tau)$ for $\operatorname{Re}=75 ;(+), C_{l}$ of the upper airfoil; $(\times), C_{l}$ of the lower airfoil; (-), $C_{l}$ of a single plunging airfoil. . . . . . . . . . . . . . . . .

Figure 8.8 Instantaneous drag coefficient $\left(C_{d}\right)$ versus nondimensional time $(\tau)$ for $\operatorname{Re}=75 ;(+), C_{d}$ of the upper and lower airfoils; (-), $C_{d}$ of a single plunging

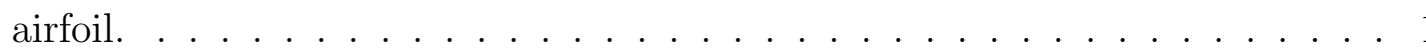


Figure 8.9 Instantaneous lift coefficient $\left(C_{l}\right)$ of the upper airfoil versus nondimensional time $(\tau) ;(*), \operatorname{Re}=75 ;(+), \operatorname{Re}=150 ;(\times), \operatorname{Re}=300 ;(\diamond), \operatorname{Re}=$ 600.

Figure 8.10 Instantaneous drag coefficient $\left(C_{d}\right)$ of the upper airfoil versus nondimensional time $(\tau) ;\left(^{*}\right), \operatorname{Re}=75 ;(+), \operatorname{Re}=150 ;(\times), \operatorname{Re}=300 ;(\diamond), \operatorname{Re}=$ 600.

Figure 8.11 Vortical patterns around the airfoils when the first $C_{d_{\max }}$ is obtained;

$$
\text { (a), } \operatorname{Re}=75 ;(\mathrm{b}) \operatorname{Re}=150 ;(\mathrm{c}), \operatorname{Re}=300 ;(\mathrm{d}), \operatorname{Re}=600 \text {. }
$$

Figure 8.12 Vortical patterns around the airfoils when the first $C_{d_{\min }}$ is obtained;

(a), $\operatorname{Re}=75 ;(b) \operatorname{Re}=150 ;(c), \operatorname{Re}=300 ;(d), \operatorname{Re}=600$.

Figure 8.13 Instantaneous lift coefficient $\left(C_{l}\right)$ versus nondimensional time $(\tau)$ for the upper airfoil: $\left(^{*}\right) f=0.25,(+) f=0.5,(\times) f=1,(\diamond) f=2 \ldots \ldots$

Figure 8.14 Instantaneous drag coefficient $\left(C_{d}\right)$ versus nondimensional time $(\tau)$ for the upper airfoil: $(*) f=0.25,(+) f=0.5,(\times) f=1,(\diamond) f=2 \ldots \ldots$

Figure 8.15 Vortical patterns around the airfoils when the first $C_{d_{\min }}$ is obtained;

(a), $f=0.25 ;(\mathrm{b}), f=0.5 ;(\mathrm{c}), f=1 ;(\mathrm{d}), f=2 \ldots \ldots \ldots$

Figure 8.16 Instantaneous lift coefficient $\left(C_{l}\right)$ versus nondimensional time $(\tau)$ for the upper airfoil: $(+) Y_{0}=0.3 c,(\times) Y_{0}=0.5 c \ldots \ldots . \ldots . . . . . . .147$

Figure 8.17 Flow field around the airfoils; (a), $C_{l_{\min }}$ at $Y_{0}=0.3 c$; (b), $C_{l_{\min }}$ at $Y_{0}$ $=0.5 c ;(\mathrm{c}), C_{l_{\max }}$ at $Y_{0}=0.3 c ;(\mathrm{d}), C_{l_{\max }}$ at $Y_{0}=0.5 c \ldots \ldots$

Figure 8.18 Instantaneous drag coefficient $\left(C_{d}\right)$ versus nondimensional time $(\tau)$ for the upper airfoil: $(+) Y_{0}=0.3 c,(\times) Y_{0}=0.5 c \ldots \ldots \ldots$

Figure 8.19 Flow field around the airfoils; (a), $C_{d_{\text {min }}}$ at $Y_{0}=0.3 c$; (b), $C_{d_{\text {min }}}$ at $Y_{0}$ $=0.5 c ;(\mathrm{c})$, second $C_{d_{\max }}$ at $Y_{0}=0.3 c ;(\mathrm{d})$, second $C_{d_{\max }}$ at $Y_{0}=0.5 c \ldots$.

Figure 8.20 Instantaneous lift coefficient $\left(C_{l}\right)$ versus nondimensional time $(\tau)$ for the upper airfoil at $g=3 c:(*) Y_{0}=0.5 c,(+) Y_{0}=0.7 c,(\times) Y_{0}=0.9 c \ldots$ 
Figure 8.21 Instantaneous drag coefficient $\left(C_{d}\right)$ versus nondimensional time $(\tau)$ for the upper airfoil at $g=3 c:(*) Y_{0}=0.5 c,(+) Y_{0}=0.7 c,(\times) Y_{0}=0.9 c \ldots$.

Figure 8.22 Instantaneous lift coefficient $\left(C_{l}\right)$ versus nondimensional time $(\tau)$ for the upper airfoil: $(+) g=1.5 c,(\times) g=3 c . \ldots \ldots \ldots 1$

Figure 8.23 Instantaneous drag coefficient $\left(C_{d}\right)$ versus nondimensional time $(\tau)$ for the upper airfoil: $(+) g=1.5 c,(\times) g=3 c \ldots \ldots \ldots$

Figure 9.1 Airfoils in the computational domain and their kinematics. . . . . . . 156

Figure 9.2 Instantaneous lift coefficient $\left(C_{l}\right)$ versus nondimensional time $(\tau)$ of for the flapping motion following Equations 6.6: (-), present simulation with the developed library; (+), Bos et al. [88]; (×), Wang et al. [58]; (o), Amiralaei et al. [6] using Laplace mesh motion algorithm. . . . . . . . . . . . . . . . .

Figure 9.3 Instantaneous lift coefficient $\left(C_{l}\right)$ versus nondimensional time $(\tau)$ for $R=1.4 c, d=0.7 c, f=0.25, \alpha_{0}=90^{\circ}, \bar{\alpha}=45^{\circ}, \phi=0$ and $\operatorname{Re}=75$ following Equations 6.2: (-), present simulations with the developed motion library; $(+)$, Amiralaei et al. [6]. . . . . . . . . . . . . . . . . .

Figure 9.4 Schematic of the airfoils and the computational domain. . . . . . . . 160

Figure 9.5 Instantaneous lift coefficient $\left(C_{l}\right)$ versus nondimensional time $(\tau)$ for the upper airfoil at $\bar{\alpha}=15^{\circ}$.

Figure 9.6 Instantaneous drag coefficient $\left(C_{d}\right)$ versus nondimensional time $(\tau)$ for the upper airfoil at $\bar{\alpha}=15^{\circ}$.

Figure 9.7 Instantaneous lift coefficient $\left(C_{l}\right)$ versus nondimensional time $(\tau)$ for the lower airfoil at $\bar{\alpha}=15^{\circ} \ldots \ldots \ldots \ldots$. . . . . . . . . . . . . . . .

Figure 9.8 Instantaneous drag coefficient $\left(C_{d}\right)$ versus nondimensional time $(\tau)$ for the lower airfoil at $\bar{\alpha}=15^{\circ} \ldots \ldots \ldots \ldots \ldots$. . . . . . . . . . . . . . . . .

Figure 9.9 Instantaneous lift coefficient $\left(C_{l}\right)$ versus nondimensional time $(\tau)$ for the upper and lower airfoils at $\bar{\alpha}=15^{\circ}$, the lower airfoil $C_{l}$ are shifted 4 units downward. 
Figure 9.10 Instantaneous drag coefficient $\left(C_{d}\right)$ versus nondimensional time $(\tau)$ for the upper and lower airfoils at $\bar{\alpha}=15^{\circ}$, the lower airfoil $C_{d}$ are shifted 10 units downward. . . . . . . . . . . . . . . . . . 164

Figure 9.11 Instantaneous lift coefficient $\left(C_{l}\right)$ versus nondimensional time $(\tau)$ for the lower airfoil at $\bar{\alpha}=30^{\circ} \ldots \ldots \ldots$. . . . . . . . . . . . . . . 165

Figure 9.12 Instantaneous drag coefficient $\left(C_{d}\right)$ versus nondimensional time $(\tau)$ for the lower airfoil at $\bar{\alpha}=30^{\circ} \ldots \ldots \ldots$. . . . . . . . . . . . . . . . . 166

Figure 9.13 Instantaneous lift coefficient $\left(C_{l}\right)$ versus nondimensional time $(\tau)$ for the lower airfoil at $\bar{\alpha}=45^{\circ} \ldots \ldots \ldots$. . . . . . . . . . . . . . . . 166

Figure 9.14 Instantaneous drag coefficient $\left(C_{d}\right)$ versus nondimensional $(\tau)$ for lower airfoil at $\bar{\alpha}=45^{\circ} \ldots \ldots \ldots \ldots$. . . . . . . . . . . . . . . 167

Figure A.1 The schematic of the airfoils and entries in the dynamicMeshDict file. 178 


\section{Nomenclature}

$\begin{array}{ll}\text { ALE } & \text { Arbitrary Lagrangian-Eulerian } \\ \text { CDS } & \text { Central differencing scheme } \\ \text { CFD } & \text { Computational fluid dynamics } \\ \text { DPIV } & \text { Digital particle image velocimetry } \\ \text { FEM } & \text { Finite element method } \\ \text { FSI } & \text { Fluid structure interaction } \\ \text { FVM } & \text { Finite volume method } \\ \text { GGI } & \text { Generalized grid interface } \\ \text { IB } & \text { Immersed boundary } \\ \text { IC } & \text { Incomplete Cholesky } \\ \text { KC } & \text { Keulegan-Carpenter number } \\ \text { LDV } & \text { Laser doppler velocimetry } \\ \text { LE } & \text { Leading edge } \\ \text { LEV } & \text { Leading edge vortex } \\ \text { LRN } & \text { Low Reynolds number } \\ \text { LU } & \text { Lower-upper } \\ \text { MAV } & \text { Micro aerial vehicle } \\ \text { N-S } & \text { Navier-Stokes } \\ \text { PBiCG } & \text { Preconditioned Biconjugate gradient } \\ \text { PCG } & \text { Preconditioned conjugate gradient } \\ \text { PDE } & \text { Partial differential equation } \\ \text { PIV } & \text { Particle image velocimetry } \\ \text { PImplicit and splitting of operators } \\ \text { PE }\end{array}$




$\begin{array}{ll}\text { RBF } & \text { Radial basis function } \\ \text { SBR } & \text { Solid body rotation } \\ \text { SCL } & \text { Space conservation law } \\ \text { SIMPLE } & \text { Semi implicit method for pressure linked equations } \\ \text { TE } & \text { Trailing edge } \\ \text { TEV } & \text { Trailing edge vortex } \\ b & \text { half-chord length }(b=c / 2) \\ c & \text { airfoil chord-length } \\ C(k) & \text { Theodorsen's function } \\ C_{d} & \text { Drag coefficient } \\ C_{d_{\max }} / C_{d_{\min }} & \text { Maximum/minimum drag coefficient } \\ \bar{C}_{d} & \text { Mean drag coefficient } \\ C_{l} & \text { Lift coefficient } \\ C_{l_{\max }} / C_{l_{\min }} & \text { Maximum/minimum lift coefficient } \\ \bar{C}_{l} & \text { Mean lift coefficient } \\ C_{m} & \text { Pitching moment coefficient } \\ C_{m_{\max }} / C_{m_{\min }} & \text { Maximum/minimum pitching moment coefficient } \\ C_{n} & \text { Normal force coefficient } \\ \bar{C}_{l} & \text { Aerodynamic performance } \\ \bar{C}_{d} & \text { Courant number } \\ d & \text { Amplitude of pitching oscillations } \\ d, R & \text { Franslational amplitudes of oscillations } \\ f & \end{array}$




\begin{tabular}{ll}
$F(k)$ & Real part of Theodorsen's function \\
$g$ & Airfoil centre-to-centre distance (airfoil spacing) \\
$G(k)$ & Imaginary part of Theodorsen's function \\
$k$ & Reduced frequency $\left(k=\frac{\pi f c}{U_{\infty}}\right)$ \\
$L_{r e f}$ & Reference length \\
$p$ & Static pressure \\
$\bar{q}$ & Mean dynamic pressure \\
Re & Reynolds number \\
St & Strouhal number \\
$S_{M}$ & Vector of source term \\
$t$ & Physical time \\
$t_{r e f}$ & Reference time \\
$t / c$ & Airfoil thickness ratio \\
$T$ & Period of oscillations \\
$U$ & Velocity vector ( $u, v, w)$ \\
$U_{r e f}$ & Reference velocity \\
$U_{0} / U_{\infty}$ & Freestream velocity \\
$x / c$ & Pitching axis location \\
$X_{M C}$ & Mid-chord position vector \\
$Y_{0}$ & Amplitude of plunging oscillations \\
$\alpha$ & Angle of attack \\
$\alpha_{0}$ & Mean angle of attack \\
$\bar{\alpha}$ & Amplitude of pitching oscillations \\
$\gamma$ & Diffusion coefficient in Laplace equation \\
& \\
\hline &
\end{tabular}


$\theta \quad$ Pitching amplitude of oscillations

$\theta_{w} / \theta_{g} \quad$ Angular variations of wing/shaft

$\lambda \quad$ Second dynamic viscosity coefficient

$\mu \quad$ First dynamic viscosity coefficient

$\nu \quad$ Kinematic viscosity coefficient

$\rho \quad$ Density

$\tau \quad$ Nondimensional time

$\phi \quad$ Phase angle between pitching and plunging motions

$\omega \quad$ Angular velocity 


\section{Chapter 1}

\section{Introduction}

\section{$1.1 \quad$ Overview}

Ever-increasing efficiency and budget constraints highlight the necessity of highly accurate solution methods for applications such as low Reynolds number (LRN) flight, fish-like robot locomotion, offshore structures and the respective fluid-structure interaction (FSI) problems. The nature of the LRN fluid phenomena differs widely from those seen in conventional intermediate and high Reynolds number cases. Often, the operation of these applications encounters complicated unsteady conditions, which could be a combination of harmonic pitching, plunging and flapping oscillations as well as aperiodic fluctuations and revolving motions. Efficient design of unsteady LRN applications, usually referred to Reynolds numbers of $\mathcal{O}\left(10-10^{4}\right)$, needs a thorough understanding of their flow physics. However, these applications mostly face or generate vortical patterns and viscous structures, adding to the inherent complexity of the flow field. More studies have been recently conducted to investigate the details of these flows. Consequently, the mechanism of the respective fluid phenomena are understood and documented in the literature [1-4], but there are still open questions about, for instance, the stability of LEVs in the LRN regime. The required in-

sight about the corresponding flow characteristics under different operating conditions and 
their dependence on the governing flow and system parameters need more investigations, especially when it comes to the analysis of instantaneous force and moment signatures. Therefore, further studies into these flows are required to contribute to the required knowledge. These investigations are beneficial for improving the existing designs and assisting in the development of new methodologies in the LRN discipline.

\subsection{Motivation}

The present research was originally initiated from an existing blade configuration employing simultaneous rotating and flapping motions to provide sustainable hovering flight [5]. This state-of-the-art mechanism is shown in Fig. 1.1.

This mechanism's two-dimensional (2-D) counterpart kinematics is a novel figure-of-

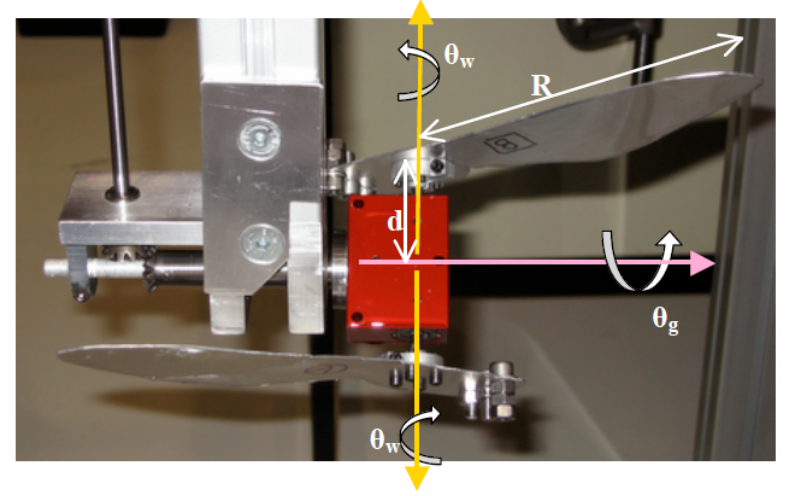

Figure 1.1: The rotating-flapping mechanism, adopted from Ania [5].

eight-like flapping pattern of each cross section [6], for which different types of unsteady conditions namely pitching, plunging and flapping of single and paired airfoils are present. The current study was then motivated by the fact that there is a wide gap between the required insight and existing knowledge in LRN airfoils/wings under unsteady motions. This gap is even more noticeable when two or more airfoils are in relative unsteady motion, where the airfoil-airfoil interactions alter the flow physics.

Forced-induced oscillations in MAVs and fish-like robots and flow-induced excitations in 
offshore structures and similar applications play a dominant role in exploiting LRN highly viscous phenomena, which lead to significant force and moment signatures. The successful design and modeling of such applications strongly depend on the accurate knowledge of these mechanisms, their dependence on the governing parameters and the variation of fluid forces and moments under different conditions, which unfortunately are not studied well.

LRN flows diverge from conventional applications in terms of velocity, characteristic dimensions, surrounding viscosity and density and the respective fluid phenomena. As most of the LRN applications run into rapid unsteady motions, the flow field is highly complex, vastly unsteady and mostly viscous. Also, the occurrence of the respective aerodynamic mechanisms and their strength/significance differ, depending on numerous factors such as kinematics and governing conditions, further increasing the degree of complexity of the problem.

Recent LRN unsteady studies have mostly utilized experimental and numerical techniques. The capability of analytical methods is restricted to special cases of extremely simplified flow conditions; hence, they lack LRN applications' desired accuracy and are not well-suited to solve these flows [7]. Quasi-steady solution, panel and Wagner's methods or Theodorsen's method for two dimensional (2-D) harmonic pitching and plunging oscillations [8] are amongst the mostly utilized analytical methods. Experimental studies provide reliable results, but they are rather expensive in terms of the required precision and testing equipment. Also, through the existing experimental techniques, the flow field cannot be visualized in great details and usually only a limited number of flow characteristics can be measured. Advanced visualization techniques such as PIV (Particle Image Velocimetry) and LDV (Laser Doppler Velocimetry) [9] are commonly used for these studies. Computational Fluid Dynamics (CFD) is another solution technique which has attracted a great attention in LRN unsteady studies. The CFD-based methods can yield accurate solutions and the flow structure can be explored in detail through numerical simulations [10].

The present study numerically investigates the LRN flow field of single/paired airfoil con- 
figurations under forced-induced oscillations. To do so, different mesh motion algorithms are utilized and developed to investigate the respective motions, aimed to improve the understanding of unsteady LRN flow fields and phenomena through extensive parametric studies. These investigations contribute to the required knowledge in LRN flows and are useful in having more efficient LRN applications from a preliminary design step perspective.

\section{$1.3 \quad$ Objectives}

The objectives of the present study are divided into three main categories as the following:

\subsubsection{Modeling airfoils under different LRN unsteady conditions}

Thorough numerical investigations of airfoils at LRN regime under prevalent unsteady motions are conducted in this thesis. The conditions include pitching, plunging and a novel figure-of-eight-like flapping motion for single and pairs of the considered airfoils. These detailed computational studies contribute to the required insight in LRN flows by showing how the kinematics affect the flow, while analytical methods have limitations and experimental means are expensive.

\subsubsection{Extensive parametric studies}

In this thesis, extensive two-dimensional (2-D) parametric studies on the effect of the governing flow and system parameters on the force and moment signatures are conducted in each investigated unsteady motion. These 2-D investigations, more appropriate for parametric sensitivity analyses than 3-D studies, will help in understanding the significance of the complex viscous phenomena under different governing flow and system parameters. The dependence of the flow physics and forces and moments on the governing conditions will also be revealed, helping LRN application designer by providing a conceptual overview of all the fluid phenomena in the application as well as the effect of these parameters on the 
fluid force generation. Moreover, the conducted 2-D parametric studies are useful if they are compared with the respective 3-D results, revealing the significance of $3-\mathrm{D}$ effects such as spanwise vortices and downwash effects.

\subsubsection{Implementation, validation and modification of the fast and robust mesh motion algorithms}

In the present research, both single and multi airfoil arrangements are studied. In the case of multi airfoils, the basic topological mesh motion algorithms in OpenFOAM ${ }^{\circledR}$ are adapted and modified to investigate the motion of multi airfoils under pitching, plunging and the novel figure-of-eight-like flapping motions. The LRN unsteady aerodynamics of moving boundary problems, in both single and multi airfoil arrangements, needs accurate and robust mesh motion algorithms. The moving mesh methods have to be capable of preserving an initially valid mesh and maintain the mesh quality (orthogonality and skewness) throughout the simulations. In the case of a pair of airfoils interacting in an unsteady motion, the existing motion algorithms are modified and new motion libraries are created to perform the motion.

\subsection{Thesis Contributions}

In this thesis, numerical simulations of single- and paired-airfoil arrangements under unsteady LRN flow conditions are performed. Extensive parametric studies in each case are also conducted. The paired-airfoil studies are simulated by developing relatively new mesh motion methodologies. The contributions of this research are categorized into the following two topics: 


\subsubsection{Analysis of flow field characteristics and force signatures of airfoils under unsteady conditions}

The 2-D simulations throughout this thesis are accompanied by extensive 2-D parametric studies, which may provide valuable information about the respective 3 -D unsteady wings/blades [58]. The conducted parametric studies reveal important information about influential flow and system parameters and their effects on the performance characteristics, namely lift, drag and moment signatures of the airfoils under unsteady motion. The present results may also be used in the respective 3-D studies, when the 2-D and 3-D results will be compared and the importance of 3-D effects and vortical interactions can be investigated. The flow structures are also explored at the points of interest, e.g. maximum lift coefficient, showing how the viscous phenomena behave and affect the flow field; hence contributing to unsteady LRN flows sought insight. In Chapter 4, for example, it is shown how reduced frequency affects the flow by delaying the initiation and separation of vortices at the trailing edge of a pitching airfoil, and that a higher reduced frequency weakens the strength of these vortices and reduces the number of the generated ones. In Chapter 5 , it is shown that despite having a symmetrical plunging kinematics the calculated lift and drag can have an asymmetrical pattern, based on the governing Reynolds (Re) and Strouhal (St) numbers. An initially drag-producing plunging airfoil can produce thrust depending on the governing Re ans st. LEVs, TEVs and very complex interaction between the plunging airfoil's surrounding vortices could explain these changes.

In Chapter 6, a novel figure-of-eight-like flapping kinematics (motivated by RotaFlap mechanism [5]) is thoroughly investigated and the corresponding parametric study reveals important information about its force and moment coefficients as well as flow structures. Most of the hovering kinematic patterns found in the literature are elliptical, non-crossing parabolic-like or almost horizontal figure-of-eight patterns [5], while a very inclined asymmetrical figure-of-eight flapping kinematics is used in this study. In the horizontally flat patterns, the vertical motion is negligible compared to the horizontal portion; hence, it does 
not contribute to the force generation significantly. However, in this figure-of-eight the vertical motion plays a substantial role in the generation of the unsteady forces. This feature of the model allows for the contribution of lift and drag forces in vertical lift resulting in more efficient upstrokes [5]. Another aspect of this kinematics is the simultaneous rotation and significant vertical translation at the end of each stroke, which could improve fluid dynamic efficiency. It is shown, for example, that this novel kinematics generates higher lift through its flapping cycle compared to the existing harmonic flapping patterns in the literature.

The multi-airfoil studies in this thesis show the significance of airfoil-airfoil interaction to the instantaneous fluid forces. The force signatures of a single plunging airfoil are compared with those of the corresponding paired-airfoils arrangement, and it is observed that the interaction changes the magnitude of the instantaneous force coefficients. Detailed examination shows that these changes are accompanied with complex flow patterns and vortical interactions. The effect of the airfoil-airfoil interaction is not always only quantitative, and it can vary the transient trend of the fluid forces, depending on the kinematic pattern. The simulations of a pair of figure-of-eight-like flapping airfoils show that the instantaneous forces of each of the simulated airfoils differ both quantitatively and qualitatively from those of a single figure-of-eight flapping airfoil.

\subsubsection{Development of mesh motion methodologies for simulating multi-body problems}

Relatively new methodologies are developed based on layer addition/removal and Generalized Grid Interface (GGI) algorithms to perform pitching, plunging and figure-of-eight flapping motions of a pair of airfoils. Although the developed motion libraries are applied towards paired-airfoil arrangements, they can also be applied to more than two moving objects, and any kinematics different than those studied in this research may also be investigated. Moreover, the developed motion methodologies can be used for the simulation of single moving geometries with significant gain in the computational time. For example, 
the harmonic and figure-of-eight-like flapping motions of a thin airfoil are simulated using the developed methodology in Chapter 9. The calculated lift coefficients are compared with those obtained from Laplace smoothing mesh motion algorithm. The results are within reasonably close agreement (5\% of difference in the mean values), while the computational time is almost $125 \%$ to $150 \%$ (depending on the kinematics and governing parameters) times bigger in the Laplacian method compared to that of the developed methodology.

\subsection{Thesis organization}

The present thesis is divided into ten chapters. The present chapter is an overview of the problem in hand, motivation, contribution and the objectives of the study. In chapter 2, the physics of LRN flows, the research endeavors in this area and different existing solution methods are presented. Chapter 3 is designated to provide detailed information on the numerical solution method, governing equations, FVM discretization, solution and mesh motion algorithms available and those used in this study. Chapter 4 presents the results of the LRN pitching study conducted on a 2-D airfoil and the investigation on the effects of the governing flow and system parameters on its instantaneous force coefficients. Chapter 5 stages the study on a plunging airfoil and the conducted parametric study. Chapter 6 presents the numerical study of the novel figure-of-eight flapping mechanism and the motivation behind such a kinematics. An extensive parametric study is conducted and details of the generated vortical patterns and their effects on the flow field are also discussed in detail. Chapters 7, 8 and 9 are dedicated to paired airfoils in pitching, plunging and figure-of-eightlike flapping motions, respectively, using mesh topological changes for the motion, followed by the conclusions and suggested future work in Chapter 10 . 


\section{Chapter 2}

\section{Background and literature review}

In the present chapter an overview of the most prevalent LRN phenomena and the respective flow physics in the operation of unsteady LRN airfoils and wings is presented. Moreover, a review of the recent research endeavors and findings available in the literature are categorized based on the three main solution approaches: analytical, experimental and numerical techniques.

\subsection{LRN flow physics}

The flow field of unsteady airfoils/wings in LRN flow regime is associated with complex nonlinear vortex shedding and viscous phenomena. These force generation mechanisms are briefly addressed in this section. The LRN respective phenomena are mostly viscous, except the added mass effect [11], and can be classified as dynamic stall and leading edge vortices [1], rotational circulation (forces), wake-capturing mechanism [3] and clap and fling mechanism [4]. In the following, a brief overview of some of the most important LRN viscous phenomena are presented. More details about these phenomena and the ones that are not discussed here are provided in [12]. 


\subsubsection{Dynamic stall and leading edge vortex}

LRN Dynamic stall [1] is referred to the generation of a leading edge vortex (LEV), also shown in Fig. 2.1, which remains attached to the airfoil surface in translational motion, and is very similar to what is observed for unsteady moving bodies at high angles of attack $[8,13]$. This usually occurs when the body translates with a high angle of attack. The flow reaches the translating body and separates from the airfoil surface at its leading edge, but reattaches at a further point downstream. Consequently, a LEV is generated which provides a low pressure zone on the airfoil upper portion, enhancing lift noticeably [2]. The LEV remains attached to the airfoil surface for some chord-lengths of travel, and sheds into the downstream wake in the following strokes. The repetition of LEV generation and shedding process may lead to the formation of periodic downstream vortical patterns such as Von Karman Vortex street [14] or chaotic vortical patterns, the type of which depends on the kinematics and governing conditions.

The stability of three-dimensional (3-D) LEVs is still not fully understood and seems to strongly depend on the governing conditions. It appears that a 3-D LEV is more stable around the unsteady wings compared to the 2-D counterpart around airfoils [15,16]. Some experimental investigations have shown that this higher stability could be related to the wing spanwise flow or downwash effects $[15,17]$, which constraint the growth of the LEV, resulting in lift augmentation [15].

\subsubsection{Rotational circulation (rotational forces)}

Direct measurements have shown that the maximum (peak) forces are generated during a wing rotation and not during the translational motion [18]. One possible source for this lift augmentation, which could make up to $35 \%$ of the total lift along with wake-capturing mechanism [3], could be the wing rotation and the respective induced circulation, changing the force signatures significantly [12]. The generated rotational circulation and its strength directly depend on the rotational axis [19] as well as the phasing between the rotational 


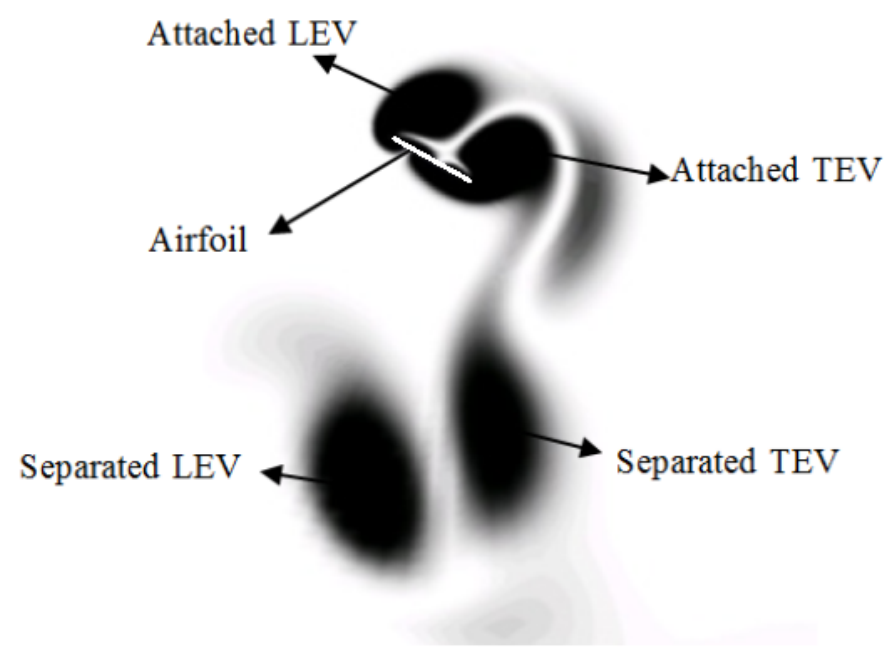

Figure 2.1: LEV and TEV around a flapping airfoil resulted from the present investigations. and translational motions in a LRN flapping/wing [3]. It is shown that advanced rotations, where the rotation is started and finished before the stroke reversal, results in higher mean lift forces than a delayed rotational case [3]. The rotational forces differ from the Kramer effect [12], which is referred to the ability of a wing to delay stall during its translational motion. 3-D numerical investigations in [20] also confirmed the significance of the rotational forces along with dynamic stall and rotational accelerations.

\subsubsection{Wake-capturing mechanism (wing-wake interaction)}

An airfoil subjected to unsteady motions such as flapping oscillations may encounter its own wake or previously separated vortices. The interaction causes the momentum exchange between the surrounding fluid and the unsteady airfoil, changing the instantaneous forces and stabilizing vortical structures around the model [12]. Dickinson et al. [3] termed this phenomenon as wake-capturing mechanism in LRN flows. However, the contribution of wakecapturing mechanism depends on the rotational forces and timing, and it could increase or even decrease the lift [21] based on the rotational phase [3]. Force augmentation during the wake-capturing mechanism is also observed in the Digital Particle Image Velocimetry (DPIV) 
robotic experiments in [22]. Figure 2.2 shows the pressure contours around a plunging airfoil during its upstroke at two consecutive time steps. Figure 2.2(a) shows a vortex on the airfoil's upper surface, previously separated during downstroke, and Fig. 2.2(b) shows that the airfoil faces this vortex, an example of wake-capturing mechanism.

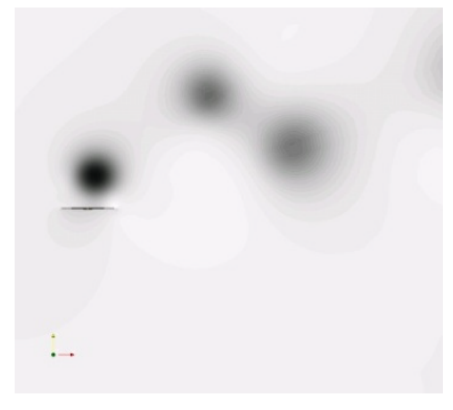

(a)

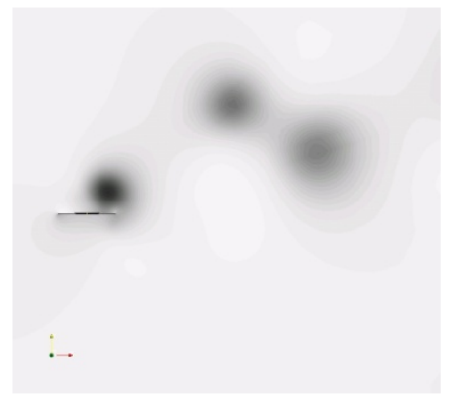

(b)

Figure 2.2: Wake-capturing mechanism during the upstroke motion of a plunging airfoil; (a) airfoil approaches the vortex; (b) airfoil encounters the vortex, corresponding to the simulations of Chapter 5 .

\subsubsection{Weis-Fogh clap and fling mechanism}

Weis-Fogh proposed a mechanism for lift enhancement in tiny wasps [4], named clap and fling mechanism or wing-wing interaction. Although this mechanism does not occur frequently in nature, it can change the force signatures significantly. The clap is referred to the instant at which two wings approach each other and their leading edges (LE) get closer, hence vortices are shed and downward wake is generated. In the fling phase [23], the wings take apart from each other having a rotation axis around their trailing edges (TE). Based on previous studies, it is suggested that the clap and fling mechanism augments the unsteady forces in flapping kinematics [12]. However, the benefit of this mechanism is still not well understood and some data [24] show that the effect of clap and fling mechanism vanishes after some angular separation between the two wings during their fling phase. 


\subsection{LRN solution methods}

Different techniques and solution methods are employed to analyze the inherently viscous flows of unsteady airfoils/wings at LRN regime. The applied methods can be categorized as analytical, experimental and numerical approaches. The available methods and studies are briefly addressed in the following subsections.

\subsubsection{Analytical methods}

Several researchers attempted to construct appropriate models for LRN unsteady flows [25, 26], however, the inherently complex and viscous LRN flows have limited the application of analytical methods. The quasi-steady approach which is founded on the basis of linear classical aerodynamic theories [27] is one of the theoretical methods in LRN unsteady flows. The force signatures are assumed to be time-invariant and the effect of the shed wake on the airfoil is not considered. Ellington [7] was one of the first researchers who applied quasisteady approach towards flapping flight and found that the method yields lower forces than those obtained from the respective experiments [7,28]. Sane and Dickinson [19] modified the quasi-steady model by adding rotational forces and added mass effects and obtained closer results to the experimental findings. However, as the LRN unsteady aerodynamic mechanisms and phenomena, e.g. dynamic stall, LEVs and wake-capturing, are not considered in the quasi-steady approach, usually the quasi-steady predictions underestimate the force measurements in experimental studies [4,29]. Therefore, quasi-steady model is not a sufficient and reliable tool for LRN unsteady studies.

The potential flow panel method [30], which is based on the inviscid flow assumption, is also used in some investigations $[31,32]$. These solutions yield reasonably good accuracy in predicting the trailing wake, whereas the close proximity of airfoil/wing flow is not resolved well due to the inviscid assumption, causing the failure to capture LRN viscous force generation mechanisms such as LEVs [33]. Unsteady aerodynamic thin airfoil theory with 
linear assumption [8] provides a foundation for some methods such as those seen in $[8,34,35]$. These methods provide the force-induced oscillation solution of 2-D, incompressible fully attached flows, with some extensions to subsonic compressible and three-dimensional rotating flows [8], having reasonable accuracy for small disturbance assumption and small and moderate reduced frequencies [36]. For example, Theodorsen's method [34] presents frequency domain solutions of pitching and plunging flat plates by modeling circulatory terms (shed wake) and non-circulatory terms (apparent mass effect), or Garrick's equations yield the thrust and peak lift coefficients [37]. But, extensive simplifications and the the lack of considering the LRN highly viscous phenomena $[1,3,4]$ encountered in LRN flows cause their break down at high amplitudes of oscillation and/or highly viscous flows [38].

\subsubsection{Experimental methods}

Experiments are performed in order to shed light on the LRN unsteady flows and their corresponding mechanisms as well as the significance of the governing flow and system parameters to the aerodynamic performance. The generated vortical patterns in LRN pitching airfoils strongly depend on the governing reduced frequency [39,40]; however Fuchiwaki and Tanaka [40] observed that the airfoil configuration and mean angle of attack do not affect these vortical patterns noticeably. Fuchiwaki et al. [41] showed the significance of the reduced frequency $(k)$ with regard to the instantaneous aerodynamic forces. The inherently complex behavior of viscous LRN unsteady flows does not merely depend on a single parameter, e.g. $k$, but a collection of governing parameters like frequency and amplitude of the oscillations. Koochesfahani's series of LRN pitching experimentations [42] demonstrated the effect of the frequency and amplitude of oscillations on the wake structures, thrust coefficients and vortex-vortex interactions, but the 3-D counterpart flow structure and other force characteristics were not investigated. Pitching-up airfoil's generated force signatures are also affected by vortex-vortex interactions, where the interactions also affect separation and dynamic stall [1]. This was explored by Shih et al. [43] PIV measurements, where they 
only considered different Reynolds numbers. Other governing parameters were not investigated.

Plunging oscillations is another prevalent unsteady condition in LRN flows. More studies are conducted on plunging (heaving) cylinders than on the plunging airfoils/wings, e.g. Bishop and Hassan [44] and Williamson and Roshko [45]. PIV investigations of Lam et al. [46] and Digital Particle Image Velocimetry visualizations of Techet et al. [47] showed that the generated vortices around heaving cylinders differ based on the imposed frequency and amplitude of oscillations as well as the Re. The flow around 2-D airfoils and their wake structures are affected by leading/trailing edge vortices (LEVs/TEVs) shown by the PIV experiments of Lua et al. [48]. These vortical patterns could be deflected with an angle with respect to the airfoil's chord-length, depending on the frequency and amplitude of the plunging oscillations [9]. TEVs [49] play a significant role in the determination of aerodynamic characteristics of 2-D plunging airfoils [50]. Freymuth [51] and Lai and Platzer [52] showed that the governing flow and system parameters such as $k$ and amplitude of oscillations change the downstream wake of 2-D plunging airfoil aerodynamic characteristics, similar to heaving cylinders' observations. Triantafyllou et al. [53] also showed that the wake could be in the form of drag producing, thrust producing or neutral patterns. Interestingly, heaving airfoils can produce thrust, developed through the formation of a reverse Von Karman vortex street [35]. In fact, the wake dynamics and its vortex topologies play a dominant role in the plunging airfoil fluid dynamics performance and efficiency.

Flapping, the most common means of force generation in LRN applications, has gained a considerable attention in the recent few years. Indeed, the exploitation of both pitching and plunging motions leads to the well-known unique unsteady and viscous mechanisms [54]. 2-D flapping airfoil experiments of Freymuth [55] showed the importance of dynamic stall in increasing thrust coefficients. 3-D studies conducted by Nagai et al. [56] also showed the significance of dynamic stall [1], rotational forces [18] and wing-wake interaction [3] in a flapping kinematics, and showed that they act differently during strokes. Flapping kinemat- 
ics parameters play a dominant role in the hovering performance [57]. Instantaneous force coefficients are some of the performance benchmarks that the knowledge of their dependence on the governing conditions yields a better insight about the flow. 3-D flapping experiments carried out by Wang et al. [58] and Singh and Chopra [59] showed the relationship between force signatures and pitching/plunging phasing, amplitude and frequency of oscillations.

In some applications, multi-moving bodies exist where the body-body interactions change the aerodynamics and vortical interactions, compared to the characteristic of a single moving body, and adds to the complexity of the corresponding fluid flow. The tube bundle of a heat exchanger, counter-rotating propellers, wings of a MAV or blades of a wind turbine are some examples in which relative oscillations could occur. To the best of the author's knowledge, the existing studies on moving bodies interactions are very limited and they are mostly on tandem and staggered cylinder arrangements. Mahir and Rockwell [61,62] experimented on the wakes of tandem/staggered cylinder arrangements at $R e=160$. In their study, the cylinders' arrangement was subjected to forced-excitation for different frequencies and different phase angles between the oscillating cylinders. They observed that the generated vortical patterns and their mechanisms vary with respect to the excitation frequency significantly. Assi et al. [63] also carried out experiments of two tandem cylinders under flow-induced oscillations. In one of the most recent experimental and numerical studies, Rival et al. [64], the aerodynamic interactions between tandem pitching and plunging airfoils was investigated using PIV for the experiments and Reynolds-averaged N-S equations for the simulations. They showed the significant effect of airfoils' vortical interactions and investigated the effects of the phase angle on the fluid forces. The tandem arrangement of two flapping wings was experimentally investigated by Warkentin and Delaurier [65]. They discovered that the tandem arrangement can lead to increased thrust and efficiency compared to a single set of flapping wings for certain relative phase angles and longitudinal spacing between the wing sets.

Experimental studies are believed to yield the most accurate results between all the avail- 
able solution methods. However, the discrete nature of testing, meaning the limited number of allowable tests for different points, makes it difficult to generalize the LRN experimental findings. Moreover, as the LRN fluid phenomena and their occurrence depend on the governing conditions, the details of these mechanisms, their strength and effectiveness still remain uninvestigated. Therefore, the other solution approach, CFD, can be an excellent candidate for investigating LRN unsteady flows in more details.

\subsubsection{Numerical methods}

Computational Fluid Dynamics (CFD) is also widely used in LRN unsteady flows to shed light on the corresponding flow characteristics. Most of the conducted numerical studies in the literature focus on three main unsteady conditions namely pitching, plunging and flapping of cylinders, airfoils and wings (objects under unsteady conditions in general), presented as the following.

\subsubsection{Pitching studies}

Highly viscous flows of LRN pitching motions and the respective phenomena cannot be resolved accurately by inviscid numerical simulations and ignoring diffusive terms [66,67]. Therefore, Navier-Stokes (N-S) equations represent the actual physics of these flows satisfying the desired accuracy. Most of the investigations on LRN 2-D and 3-D pitching motions correspond to Re order of magnitude $\mathcal{O}\left(10^{4}\right)$ and $\mathcal{O}\left(10^{5}\right)$, while lower Re studies relevant to the proposed study, $\mathcal{O}\left(10^{2}\right)$ and $\mathcal{O}\left(10^{3}\right)$, are scarce. Akbari and Price [68] utilized a vortex method to simulate the flow field around a pitching airfoil utilizing N-S equations. They examined the effects of some parameters on the instantaneous forces, and found that $k$ and Re have the maximum and minimum effects, respectively, amongst the investigated parameters. In other studies, the effects of the governing parameters on the airfoil surrounding flow field are investigated. For example, Okong' o and Knight's [69] 2-D pitching airfoil simulations showed that the main influence of Re is to reduce the angle of attack at which the primary 
recirculation region starts to develop on the leeward side of the airfoil, and higher Re causes the location of these recirculation regions to be closer to the LE. Hamdani and Sun [70] attributed large unsteady forces in their simulations to extensive generated vortical patterns around unsteady pitching airfoils. The importance of the governing flow and system parameters such as the frequency and amplitude of oscillations, pitching axis location and pitching rate on the generated forces and vortical patterns were shown by Young and Lai [71] and Visbal and Shang [72]. Visbal and Shang [72] also showed that the highly unsteady flow structures are accompanied by reverse flow in the boundary layer, shedding of TEVs into downstream and formation of LEV with a shear layer on the leeward section of the airfoil.

\subsubsection{Plunging studies}

Plunging motion is another prevalent unsteady condition observed in LRN flows. Plunging cylinders have gained more attention than plunging airfoils due to their higher number of applications. For example, Leontini et al. [73], Marzouk [74] and Yang and Zheng [75] numerically investigated plunging cylinders downstream wakes and their dependence on the governing conditions. Andro and Jacquin [76] simulated the flow field of a heaving airfoil, and analyzed the significance of LEVs, added mass [11] and wake-capturing [3] mechanisms to the flow structure. They studied the effect of the frequency of oscillations on the strength and effectiveness of these mechanisms, and classified their findings into three categories: quasi steady, transitional and added mass regimes at which LEV, wake-capturing and added mass effects, respectively, are the most significant fluid phenomena. The importance of some governing parameters such as thickness ratio (thickness to chord ratio), Re and Strouhal number (St) to the flow characteristics $[77,78]$, TE geometry and ground effect to the aerodynamic forces [79] and different airfoil shapes to thrust generation and performance [80] are also shown by performing 2-D numerical studies. Viscous unsteady mechanisms also play a significant role in the determination of LRN plunging fluid forces and flow structures. Lewin and Haj-Hariri [81] explored flow characteristics of a heaving airfoil numerically, calculated 
power and thrust coefficients and examined the effects of LEV/TEV and their interaction on the aerodynamic efficiency. They also showed that the optimal efficiency is obtained for an intermediate Strouhal number (St), and that the LEV separation leads to decreased thrust and efficiency. Pederzani and Haj-Hariri [82] simulated the flow field of a chordwise flexible heaving airfoil and found that it is more aerodynamically efficient than a rigid airfoil. 2-D simulations of Young and Lai [71] showed that the wake structure and lift and thrust of a plunging airfoil strongly depend on reduced frequency and St, and displayed the remarkable influence of leading edge separation and trailing edge effects on the flow field. Young and Lai [83] conducted simulations of a 2-D transversally oscillating airfoil and observed asymmetric vortex lock-in phenomenon, which is prevalent in oscillating cylinders.

\subsubsection{Flapping studies}

More studies have investigated LRN flapping motions than LRN pitching and plunging oscillations. Vortical patterns affect the instantaneous forces in a flapping flight. Lee et al. [84] investigated the role of LEVs/TEVs in force generation and concluded that LEVs affect thrust beside their known effect on increasing lift. Force generation mechanisms act differently during flapping up and down strokes [3]. Mittal et al. [85] numerically simulated a flapping airfoil flow field and concluded that the formation of an inverse Karman vortex street is accompanied with the highest thrust efficiency. The 2-D figure-of-eight-like motion simulations of Lee et al. [86] showed that lift is mainly produced during downstrokes, while thrust is mostly achieved at the end of upstrokes, where they attributed the generated thrust to vortex pairing, which is different from the conventional inverse Karman vortex street found in sinusoidal flapping motions [85]. The significance of delayed stall and wakecapturing mechanism in flapping airfoils as well as the effects of Re and reduced frequency were investigated by Tang et al. [87]. The influence of flapping kinematics on the aerodynamic performance of flapping airfoils are also studied by Tang et al. [87] and Bos et al. [88]. System parameters such as plunging amplitude and frequency of oscillations were shown 
by Kinsey and Dumas [89] to have the highest impact on the instantaneous fluid forces. They noticed that higher lift is produced during advanced rotations, where the pitching oscillation starts before the end of each plunging stroke. Kaya and Tuncer [90] studied a nonsinusoidal flapping pattern and performed an optimization study on the kinematics to obtain maximum thrust and/or propulsive efficiency. In their 2-D numerical simulation, they observed that the maximum thrust coefficient can be obtained if the airfoil stays at almost constant angle of attack during up and down strokes, and the pitching occurs at the end of each stroke. However this is achieved at very high acceleration rates in both pitch and plunge, and at the expense of a lower propulsive efficiency. Sun and Tang [91] simulated a 3-D hovering wing using N-S equations and examined advanced, symmetrical and delayed rotation cases. Young et al. [92] conducted simulations of a 3-D flapping wing and performed a parametric study. They concluded that both lift and thrust are influenced by the frequency of oscillations. In another study, Dong et al. [93] explored the wake topology of a finite aspect ratio wing performing a harmonic flapping motion and reconfirmed that the topology is different from those of infinitely long wings. They also studied the effects of Re and St on the wing's performance and investigated their importance to the unsteady forces. Ramamurti and Sandberg [94] performed 3-D Finite Element Method (FEM) based simulations of flapping motion and studied the effect of phase angle between rotational and translational motions on the instantaneous forces. They observed that advanced rotations yield higher thrust coefficients than symmetrical and delayed cases.

Ramamurti and Sandberg [95] also investigated 3-D flapping motions under different conditions such as gust loading and flexibility and compared lift and thrust coefficients as the performance benchmarks. Chern et al. [96] simulated flow patterns of a pair of side-by-side square cylinders. They prescribed harmonic oscillatory motion to cylinders and investigated the generated flow patterns. They evaluated the effects of Keulegan-Carpenter (KC) number (a nondimensional number defined as the ratio of drag to inertia forces), Reynolds number and cylinder gap spacing on the surrounding flow, and concluded that Re has fewer effects 
on the flow physics than KC and gap spacing. Lee et al. [97] used an immersed boundary method to simulate the flow around two side-by-side oscillating cylinders at Re $=185$. They investigated the effect of the gap between two cylinders and the frequency of oscillations on the wake topology and instantaneous force coefficients of the cylinders. They concluded that two oscillating cylinders have similar flow characteristics to two stationary cylinders as well as a single oscillating cylinder. Mittal and Kumar [98] used a Finite Element formulation to study the flow-induced oscillations between two tandem and staggered cylinders at LRN regime. They used cylinder spacing such that the flow-induced oscillations occurred in wakeinference regime, where the cylinders are within close distance with respect to each other and the wake of one cylinder affects the other cylinder flow patterns. They observed that the downstream cylinder undergoes very large amplitude oscillations. The numerical simulations of Papaioannou et al. [99] showed the effect of spacing in a tandem arrangement under vortex-induced oscillations. They observed that the response region of the upstream cylinder is widened when spacing decreases. They also showed that the amplitude of vortex-induced oscillations depends on the spacing. Prasanth and Mittal [100] used FEM to study the 2-D flow-induced oscillations of two tandem/staggered cylinders at low Reynolds numbers. They allowed the cylinders to move in both in-flow and cross-flow directions. Singha and Sinhamahapatra [101] simulated the flow field of two stationary tandem cylinders at LRN regime using FVM. They studied the effect of Reynolds number and gap spacing and found that these two parameters affect the flow field significantly. Jester and Kallinderis [102] numerically investigated the incompressible flow about transversally oscillating cylinder pairs, in both tandem and side-by-side arrangements, and explored the effect of phase angle on the wake structures of the cylinder pairs. Akhtar et al. [103] simulated the effect of an upstream flapping foil on the thrust generation of a similar downstream flapping foil and found that this vortical interaction is capable of increasing the thrust generation of the downstream flapping foil. They also concluded that the thrust augmentation depends on the phase angle between the two flapping motions. 
This chapter presented different existing techniques to investigate LRN flows along with some of the most important findings in LRN flow literature. The methods span the analytical, experimental and numerical means, where each of these methods have their own pros and cons. As the focus of the present thesis is on CFD with a finite volume approach, the next chapter is devoted to the details of the existing CFD methods for discretization of the N-S equations, solving discretized governing equations and mesh motion algorithms necessary to study unsteady motions. 


\section{Chapter 3}

\section{Numerical Simulation Method}

Different solution techniques in LRN flow regime were presented in Chapter 2 along with several studies available in literature. The focus of this chapter is on the numerical techniques, Computational Fluid Dynamics (CFD) with a Finite Volume Method. In what follows, governing (N-S) equations are presented, followed by the discretization schemes, boundary and initial condition treatment and matrix solution methods. Different mesh motion algorithms for investigating moving mesh problems in addition to those of the present thesis are also presented in this chapter.

\subsection{Introduction}

The starting point of the Finite Volume Method (FVM) is to write the N-S equations on an infinitesimally small control volume surrounding a fluid element, where the N-S equations are integrated over the control volume and discretized so that a system of algebraic equations for all the computational domain's nodes are obtained. Boundary and initial conditions are then applied and the obtained matrix system is solved using matrix solution methods. In the present chapter, the governing N-S equations and their discretization, boundary conditions, matrix solution methods and the pressure-velocity coupling algorithms for incompressible N-S equations are presented followed by the mesh motion algorithms for the investigated 
moving airfoils' problems. The open source code OpenFOAM ${ }^{\circledR}$ is used and the existing mesh motion methods are modified to handle the desired motions.

\subsection{Governing equations}

The governing equations of fluid flow represent the mathematical statement of conservativeness in terms of mass, momentum and energy. These conservation equations can be written for an infinitesimally small fluid particle (control volume). Versteeg and Malalasekera [105], for example, presented the details of obtaining these equations. The continuity equation (mass conservation equation) is obtained by equating the rate of increase of mass within the fluid control volume to the net rate of mass flow into the control volume, resulting in:

$$
\frac{\partial \rho}{\partial t}+\frac{\partial(\rho u)}{\partial x}+\frac{\partial(\rho v)}{\partial y}+\frac{\partial(\rho w)}{\partial z}=0
$$

where $\rho$ is the fluid density, $t$ is the physical time and $u, v$ and $w$ stand for the $x, y$ and $z$ directions velocity components. Equation 3.1 can be written in the following compact form:

$$
\frac{\partial \rho}{\partial t}+\nabla \cdot(\rho U)=0
$$

with $\nabla=(\partial / \partial x, \partial / \partial y, \partial / \partial z)$ and $U=(u, v, w)$. Equation 3.2 is the general continuity equation for compressible flows and can be simplified into the following equation for incompressible ( $\rho=$ constant) flows:

$$
\nabla \cdot(U)=\frac{\partial u}{\partial x}+\frac{\partial v}{\partial y}+\frac{\partial w}{\partial z}=0
$$

The momentum equations are derived based on the Newton's second law, stating that the rate of increase of fluid momentum within an infinitesimally small control volume is equal to the summation of the exerted forces on the control volume. The forces are generally 
divided into surface and body forces [104]. Surface forces include pressure (normal) and shear stresses, surface tension, etc., while the body forces contain gravitational, centrifugal, Coriolis, etc [104]. The application of Newton's law leads to the following 3-D momentum equations (on a space-fixed coordinate system):

$$
\begin{aligned}
\rho \frac{D u}{D t} & =\frac{\partial\left(-p+\tau_{x x}\right)}{\partial x}+\frac{\partial \tau_{y x}}{\partial y}+\frac{\partial \tau_{z x}}{\partial z}+S_{M x} \\
\rho \frac{D v}{D t} & =\frac{\partial \tau_{x y}}{\partial x}+\frac{\partial\left(-p+\tau_{y y}\right)}{\partial y}+\frac{\partial \tau_{z y}}{\partial z}+S_{M y} \\
\rho \frac{D w}{D t} & =\frac{\partial \tau_{x z}}{\partial x}+\frac{\partial \tau_{y z}}{\partial y}+\frac{\partial\left(-p+\tau_{z z}\right)}{\partial z}+S_{M z}
\end{aligned}
$$

where $p$ is static pressure, $\tau_{i j}(i$ and $j=x, y, z)$ represents viscous stresses, $S_{M}$ is the vector of source terms equal to $S_{M}=\left(S_{M_{x}}, S_{M_{y}}, S_{M_{z}}\right)=(0,0,-\rho g)$ and $D / D t$ is the substantive derivative defined as $D / D t=\partial / \partial t+U \cdot \nabla$. The viscous stresses can be modeled as a function of deformation or strain rate as the following:

$$
\begin{array}{rlrl}
\tau_{i j}=2 \mu \frac{\partial U_{i}}{\partial x_{i}}+\lambda \nabla \cdot U & i=j, \\
\tau_{i j}=\mu\left(\frac{\partial U_{i}}{\partial x_{j}}+\frac{\partial U_{j}}{\partial x_{j}}\right) & i \neq j,
\end{array}
$$

where $\mu$ and $\lambda$ are the first and second dynamic viscosity coefficients, respectively, correlated by $\lambda=-(2 / 3) \mu$ according to Stokes hypothesis [106]. Finally, by implementing the viscous stress models (Equations 3.5), the 3-D momentum equations are:

$$
\frac{\partial\left(\rho u_{i} U\right)}{\partial t}+\nabla \cdot\left(\rho u_{i} U\right)=-\frac{\partial p}{\partial x_{i}}+\nabla \cdot\left(\mu \nabla u_{i}\right)+S_{M i}
$$

where $i=1,2$ and 3 represents $x, y$ and $z$ directions.

The governing fluid flow equations also include the energy equation if the flow is compressible [104]. However, the current study is conducted for 2-D unsteady, incompressible flow (with laminar assumption). The continuity and momentum (N-S) equations under these 
assumptions are simplified into the following equations:

$$
\begin{aligned}
& \frac{\partial u_{i}}{\partial x_{i}}=0 \\
& \frac{\partial u_{i}}{\partial t}+\frac{\partial u_{i} u_{j}}{\partial x_{j}}=-\frac{1}{\rho} \frac{\partial p}{\partial x_{i}}+\nu \frac{\partial^{2} u_{i}}{\partial x_{j} \partial x_{j}}
\end{aligned}
$$

where $i=1$ and 2 represents the $x$ and $y$ coordinate for 2 -D flow, respectively, $u_{i}$ is the velocity in $i$ direction and $\nu=\frac{\mu}{\rho}$ symbolizes the kinematic viscosity. It is a common practice to nondimensionalize the governing equations in fluid dynamics studies so that the important nondimensional numbers are known and highlighted, the comparison and validation purposes can be better performed and scaling to real applications is made feasible [104]. Equations 3.7 are nondimensionalized by defining appropriate dimensionless variables as the following:

$$
u_{i}^{*}=\frac{u_{i}}{U_{r e f}} ; \quad x_{i}^{*}=\frac{x_{i}}{L_{r e f}} ; \quad t^{*}=\frac{t}{t_{r e f}} ; \quad \rho^{*}=\frac{\rho}{\rho_{\text {ref }}} ; \quad p^{*}=\frac{p}{\rho_{\text {ref }} U_{r e f}^{2}},
$$

where the variables with asterisk are the dimensionless variables. $U_{\text {ref }}$ is defined based on the problem specifications, e.g. mean airfoil velocity in a period of flapping oscillations in a quiescent environment, $L_{r e f}$ is mostly the airfoil/wing chord-length, $t_{r e f}$ is the period of oscillations and $\rho_{\text {ref }}=\rho$, hence $\rho^{*}=1$.

Substituting the taken nondimensional variables in Equations 3.8 into Equations 3.7 yields:

$$
\begin{aligned}
& \frac{\partial u_{i}^{*}}{\partial x_{i}^{*}}=0, \\
& \mathrm{St} \frac{\partial u_{i}^{*}}{\partial t^{*}}+\frac{\partial u_{i}^{*} u_{j}^{*}}{\partial x_{j}^{*}}=-\frac{\partial p^{*}}{\partial x_{i}^{*}}+\frac{1}{\operatorname{Re}} \frac{\partial^{2} u_{i}^{*}}{\partial x_{j}^{* 2}},
\end{aligned}
$$

where St and Re stand for the Strouhal and Reynolds numbers, respectively, given by: 


$$
\mathrm{St}=\frac{L_{r e f}}{U_{r e f} t_{r e f}}=\frac{f_{r e f} L_{r e f}}{U_{r e f}} ; \quad \operatorname{Re}=\frac{\rho_{r e f} U_{r e f} L_{r e f}}{\mu}=\frac{U_{r e f} L_{r e f}}{\nu}
$$

From a fluid dynamicist perspective, the effect of the governing nondimensional parameters on the instantaneous force generation and the respective flow physics of an unsteady moving airfoil is important as it reveals the possible ways of improving the performance of an existing design as well as designing a new application. Therefore, parametric studies of each investigated case in this thesis are presented to show these effects.

\subsection{Discretization of N-S equations}

The form of Equations 3.6, 3.7 and 3.9 are not appropriate for FVM as the approach discretizes the fluid equations in their integral form. Continuity, momentum and energy conservation equations can be written in the so-called general transport equation [105]:

$$
\frac{\partial(\rho \phi)}{\partial t}+\nabla \cdot(\rho \phi U)=\nabla \cdot(\Gamma \nabla \phi)+S_{\phi}
$$

where $\phi$ is 1, u,v,w and $i$ (fluid internal energy) and $\Gamma$ and $S_{\phi}$ are diffusion coefficient and source term, respectively, corresponding to the utilized $\phi$ in Equation 3.11. The key step of FVM is the integration of Equation 3.11 over an infinitesimally small control volume resulting in:

$$
\int_{C V} \frac{\partial(\rho \phi)}{\partial t} d V+\int_{C V} \nabla \cdot(\rho \phi U) d V=\int_{C V} \nabla \cdot(\Gamma \nabla \phi) d V+\int_{C V} S_{\phi} d V
$$

Applying Gauss's divergence theorem [105] is another important step in FVM discretization, converting the convective and diffusive terms' (the second and third terms in Equation 3.12 from left) volume integrals into control volume bounding surface integrals as the following: 


$$
\frac{\partial}{\partial t} \int_{C V} \rho \phi d V+\oint_{A} n \cdot(\rho \phi U) d A=\oint_{A} n \cdot(\Gamma \nabla \phi) d A+\int_{C V} S_{\phi} d V
$$

where $n$ represents unit normal vector to the control volume faces/edges.

Finally, as unsteady motions are investigated in this study, integration of Equation 3.13 with respect to time has to be taken, resulting in:

$$
\int_{\Delta t} \frac{\partial}{\partial t} \int_{C V} \rho \phi d V d t+\int_{\Delta t} \oint_{A} n \cdot(\rho \phi U) d A d t=\int_{\Delta t} \oint_{A} n \cdot(\Gamma \nabla \phi) d A d t+\int_{\Delta t} \int_{C V} S_{\phi} d V d t
$$

Using Equation 3.13, incompressible 2-D momentum equations are obtained by taking $\phi$ $=U=(u, v), \Gamma=\mu$ and $S_{\phi}=0$ (assuming no source term), as the following [105]:

$$
\int_{\Delta t} \int_{C V} \frac{\partial U}{\partial t} d V d t+\int_{\Delta t} \oint_{A} n \cdot(U U) d A d t=\int_{\Delta t} \int_{C V}\left(\frac{-\nabla p}{\rho}\right) d V d t+\int_{\Delta t} \oint_{A} n \cdot(\nu \nabla U) d A d t
$$

\subsubsection{Discretization of temporal and spatial terms in incompress- ible momentum equations}

The first terms in Equations 3.13 and 3.14 represent the rate of change of the fluid property $(\phi)$ with respect to time for unsteady flows, where a proper temporal discretization scheme has to be employed. In the case of incompressible flow, where Equation 3.14 is divided by constant density, this term can be rewritten as:

$$
\int_{\Delta t} \frac{\partial}{\partial t}\left(\int_{C V} \phi d V\right) d t=\int_{C V} \int_{\Delta t} \frac{\partial \phi}{\partial t} d t d V=\left(\int_{\Delta t} \frac{\partial \phi}{\partial t} d t\right) V_{C V}
$$

where the volume integral is approximated by the multiplication of the fluid property in the control volume center $\left(\phi_{P}\right)$ and the volume of the fluid element $\left(\int_{C V} \phi d V \approx \phi_{p} V_{C V}\right)$. The rate of change of $\phi\left(\frac{\partial \phi}{\partial t}\right)$ is discretized based on: 


$$
\begin{aligned}
& \frac{\partial \phi}{\partial t}=f(t, \phi(t)) ; \quad \phi\left(t_{0}\right)=\phi^{*} \\
& \int_{t_{n}}^{t_{n+1}} \frac{\partial \phi}{\partial t} d t=\phi^{n+1}-\phi^{n}=\int_{t_{n}}^{t_{n+1}} f(t, \phi(t)) d t=\bar{f}(t, \phi(t)) \Delta t,
\end{aligned}
$$

where $\phi^{0}$ is the initial condition [104], $\phi^{n}$ and $\phi^{n+1}$ are the old and new variables at $t_{n}$ and $t_{n+1}$ respectively and $f(t, \phi(t))$ is the discretization scheme applied to the time gradient. In the present study first and second order implicit schemes are used for temporal discretization, shown in Equations 3.18. The second order implicit method (asymmetric) in Equations 3.18 is less prone to producing oscillatory solutions than the symmetric second order CrankNicolson scheme [104], if the time step is small enough. This scheme is also unconditionally stable.

$$
\begin{aligned}
& f(t, \phi(t))=\frac{\partial \phi}{\partial t} \cong \frac{\phi^{n+1}-\phi^{n}}{\Delta t} \\
& f(t, \phi(t))=\frac{\partial \phi}{\partial t} \cong \frac{\frac{3}{2} \phi^{n+1}-2 \phi^{n}+\frac{1}{2} \phi^{n-1}}{\Delta t}
\end{aligned}
$$

The implicit schemes are preferred when explicit schemes fail to result in stable solutions [104]. However, the higher stability is achieved at the expense of the higher computational resources and longer calculation times needed for implicit schemes. Maximum Courant number is used to monitor the stability of the simulations. The relation between time step $(\Delta t)$ and a prescribed Courant number is:

$$
C o=\frac{U \Delta t}{\Delta x}
$$

where $\Delta t$ is the time step and $\frac{\Delta x}{U}$ is the time required for a disturbance to be convected a 
distance $\Delta x[104]$.

The second term in the left hand side of Equation 3.13 (divided by density for incompressible flows) is called the convective term:

$$
\int_{\Delta t} \oint_{A} n \cdot(\phi U) d A d t=\left(\oint_{A} n \cdot(\phi U) d A\right) \Delta t
$$

where $\phi=(1, u, v)$ for incompressible 2-D flow and $U=(u, v)$ is the velocity vector. The surface integral using FVM discretization is converted into the summation of normal fluxes $\left(\phi_{f} U_{f} A_{f}\right)$ over the control volume faces (denoted by $\left.f\right)[105]$ :

$$
\oint_{A} n \cdot(\phi U) d A=\sum_{f} A_{f}(U \phi)_{f}=\sum_{f} F \phi_{f}
$$

where $F=A_{f} U_{f}$ (face area times the velocity vector at the respective face) and $\phi_{f}$ is the fluid property at the face. However, in FVM, the fluid properties such as pressure and velocity components are not restored at the faces but at the control volumes' centers. Hence, $\phi_{f}$ has to be obtained using an appropriate discretization scheme. Central Differencing Scheme (CDS), [105], is used in this study for interpolating the fluid property at the faces.

Diffusive term is the first term in the right hand side of Equation 3.13. The diffusion coefficient for incompressible momentum equations (divided by density) is $\Gamma=\nu$. The diffusive term in Equation 3.13 is discretized as the following:

$$
\oint_{A} n \cdot(\Gamma \nabla \phi) d A=\oint_{A} n \cdot(\nu \nabla \phi) d A \cong \sum_{f} A_{f}(\nu \nabla \phi)_{f}=\sum_{f} \nu_{f} A_{f} \nabla \phi_{f}
$$

where $\nu_{f}$ stands for the kinematic viscosity at the cell face and is obtained by interpolating from neighbor cells' centers to the faces. The diffusive terms in this study are also discretized based on CDS [105]. 


\subsection{Boundary and initial conditions}

The present study investigates the flow field of unsteady moving airfoils in single and paired arrangements. The solution of the respective governing (N-S) equations needs the flow field properties (velocity and pressure) to be specified on the computational domain boundaries. Four different boundary conditions are used in this study.

- At the inflow boundary, the velocity is specified (velocity Dirichlet boundary condition [104]) based on the desired Re, and the pressure is restricted to the zero-gradient condition (Neumann boundary condition [104]).

- At the outflow boundary, the pressure is set to the freestream value (Dirichlet boundary condition), while the velocity is set to the zero-gradient condition (Neumann boundary condition).

- The far-field boundary is set to symmetry boundary condition [104], and is placed at an appropriate distance from the moving body surface to minimize its undesired effects on the airfoil's surrounding flow field.

- The stationary airfoils/cylinders are set to no-slip boundary condition with fixed velocity $(U=0)$ and zero gradient pressure boundary condition.

- The unsteady moving airfoils are set to moving wall velocity boundary condition in OpenFOAM ${ }^{\circledR},[107]$. This boundary condition guarantees the no-slip boundary condition by introducing an extra velocity to keep the flux through the moving boundary equal to zero.

The solution of N-S equations also needs the initial fluid properties (velocity pressure, kinematic viscosity) to be specified at the start of the simulations. The steady state solutions are used as the initial conditions for the time-marching unsteady calculations with freestream velocity. However, the unsteady solutions without freestream velocity are started from zero velocity and freestream pressure over the computational domain. 


\subsection{Solution of the governing equations}

Equations 3.7 present the governing N-S equations of the unsteady, incompressible flow around airfoils, and can be written in their extended form as:

$$
\begin{aligned}
& \frac{\partial u}{\partial x}+\frac{\partial v}{\partial y}=0 \\
& \frac{\partial u}{\partial t}+u \frac{\partial u}{\partial x}+v \frac{\partial u}{\partial y}=-\frac{1}{\rho} \frac{\partial p}{\partial x}+\nu\left(\frac{\partial^{2} u}{\partial x^{2}}+\frac{\partial^{2} u}{\partial y^{2}}\right) \\
& \frac{\partial v}{\partial t}+u \frac{\partial v}{\partial x}+v \frac{\partial v}{\partial y}=-\frac{1}{\rho} \frac{\partial p}{\partial y}+\nu\left(\frac{\partial^{2} v}{\partial x^{2}}+\frac{\partial^{2} v}{\partial y^{2}}\right)
\end{aligned}
$$

The solution of Equations 3.23 faces two challenges. First, the convective terms such as $u \partial u / \partial x$ are non-linear quantities. Second, all the equations are coupled as the velocity components appear in continuity as well as $x$ and $y$ momentum equations. Both of these problems can be resolved by adopting iterative solution strategies such as the Semi Implicit Method for Pressure Linked Equations (SIMPLE) of Patankar and Spalding [108] or the Pressure Implicit with Splitting of Operators (PISO) algorithm of Issa [109]. Both SIMPLE and PISO algorithms are founded on a similar concept. Convective fluxes $(F)$ in Equation 3.21 are evaluated using a guessed initial velocity field. A guessed pressure field is also used to solve the momentum equations. Moreover, the continuity equation is converted into a pressure correction equation [105], used for modifying the guessed pressure field. This pressure correction is used to update the velocity and pressure consequently. Both SIMPLE and PISO algorithms are used in this study, where the main difference between them is that PISO has one more corrector step than SIMPLE. SIMPLE algorithm is originally put forward by Patankar and Spalding [108] having a guess and correct procedure on staggered grids, where scalar variables such as pressure and viscosity are evaluated at ordinary nodal points and velocity components are evaluated on staggered grids centered around the cell faces [110]. However, OpenFOAM ${ }^{\circledR}$ uses a collocated grid arrangement, where all the fluid 
properties are restored as the cell centers, and the required modifications are taken into account using Rhie and Chow method [111]. The steps involved in the SIMPLE calculation, for example, are as the following:

- Discretized momentum equations are solved with a guessed value for pressure field; so, guessed values for velocity components are obtained.

- The discretized momentum equations are solved using the initially guessed values for pressure and velocity.

- The new values are used in the discretized continuity equation (pressure correction equation) to obtain pressure corrections.

- The new velocity and pressure values are evaluated and convergence is checked.

- The procedure is repeated until convergence is achieved [112].

The governing (N-S) equations are in the form of Partial Differential Equations (PDEs). After the computational domain is discretized, the N-S equations, having linear and nonlinear algebraic expressions, are written for all the mesh points, resulting in a sparse matrix [104]. The obtained algebraic equations are then arranged into a form similar to Equation 3.24, where appropriate iterative matrix methods have to be adopted to solve for theses nonlinear equations. Moreover, a convergence criterion, usually based on the residual, is specified and the iterations are conducted until the convergence criterion is met. The matrix form of the governing equations is:

$$
A \phi=Q,
$$

where $A$ is the sparse matrix obtained from the discretized N-S equations, $\phi=(1, u, v)$ in 2-D flows and $Q$ is the vector obtained applying initial and boundary conditions. Preconditioned Conjugate Gradient (PCG) solvers for pressure and mesh motion equation and Preconditioned Bistab Conjugate Gradient (PBiCG) solver [104] and [112] for pressure veloc- 
ity coupling are used in this study, where preconditioning varied from Incomplete Cholesky (IC) to LU decomposition [104].

\subsection{N-S equations for moving grid problems}

In many fluid engineering applications, boundaries move, which accordingly makes the solution domain change in time. The source of the motion can be forced oscillations such as MAVs' wing flapping or flow-induced oscillations such as wing flutter. The integrated general transport equation (Equation 3.13) for a scalar variable $\phi$ in an arbitrarily moving control volume bounded by closed surface $A_{s}$ can be rewritten as:

$$
\frac{\partial}{\partial t} \int_{V_{C V}} \rho \phi d V+\oint_{A_{s}} n \cdot\left(\rho \phi\left(U-U_{s}\right)\right) d A=\oint_{A_{s}} n \cdot(\Gamma \nabla \phi) d A+\int_{V_{C V}} S_{\phi} d V,
$$

where $U_{s}=\left(u_{s}, v_{s}, w_{s}\right)$ is the velocity vector of the control volume bounding surface and $V_{C V}$ and $A_{s}$ are the volume of the fluid element and the area of the bounding surface, respectively, correlated by the so-called Space Conservation Law (SCL), [104] and [107], as the following:

$$
\frac{\partial}{\partial t} \int_{V_{C V}} d V-\oint_{A_{s}} n \cdot U_{s} d A=0
$$

Equation 3.26 can be physically interpreted as the mass conservation equation for zero fluid velocity, describing the conservation of space when the control volume changes its shape and/or position in an arbitrarily moving problem [104].

After applying the FVM reasoning to discretize the temporal and spatial terms in Equation 3.25, the computational domain is split into a finite number of convex polyhedral cells (Fig. 3.1) and a time marching problem. For example, discretization of Equation 3.25 for a cell around point $\mathrm{P}$ (Figure 3.1) results: 


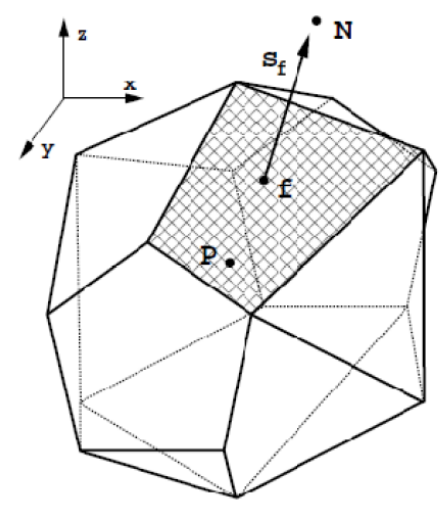

Figure 3.1: Arbitrary polyhedral cell around point $\mathrm{P}$ in the computational domain, adopted from Jasak and Tuković [107].

$$
\frac{\left(\rho_{P} \phi_{P} V_{C V}\right)^{n}-\left(\rho_{P} \phi_{P} V_{C V}\right)^{n-1}}{\Delta t}+\sum_{f} \rho_{f} \phi_{f} A_{f}\left(U-U_{s}\right)=\sum_{f} \Gamma_{f} A_{f} \nabla \phi_{f}+S_{\phi} V_{P}
$$

where $P$ and $f$ show the cell center and face in Fig. 3.1, respectively, $V_{C V}$ is the cell volume and $n$ and $n-1$ are the new and old times. The fluid flux $\left(A_{f} U\right)$ is obtained during the solution and satisfies the mass conservation in N-S equations 3.23. The mesh motion flux $\left(A_{f} U_{s}\right)$, however, has to satisfy the Space Conservation Law (SCL) presented in Equation 3.26, which is always satisfied in its integral form [107], however it also needs to be preserved in its discrete form as the following:

$$
\frac{V_{P}^{n}-V_{P}^{n-1}}{\Delta t}-\sum_{f} A_{f} U_{s}=0
$$

Therefore, the mesh motion flux is calculated based on Equation 3.28, where the volume swept [104] by the mesh face $f$ is calculated based on the geometric specifications [107]. This constraint was successfully considered and implemented in OpenFOAM ${ }^{\circledR}$ [107]. 


\subsection{Moving mesh algorithms}

There are numerous examples in fluid engineering applications, in which the boundaries move and cause the interior medium to deform, e.g. fluid-structure interaction and unsteady aerodynamics of pitching/plunging/flapping and unharmonically moving lifting surface devices. Investigation of unsteady airfoils/wings fluid dynamics needs an accurate mesh motion algorithm to account for the displacement and deformation of the computational domain interior points and cells. The mesh motion algorithm has to be capable of preserving a high quality and initially valid mesh [107], owing to the fact that the simulation accuracy strongly depends on the mesh motion solution, where the internal point motion influences the solution through mesh discretization errors [107]. This may lead to the wrong answers or even failure of the simulation if not treated properly. The most important mesh quality benchmarks are the cell orthogonality and skewness [107]. Other criteria such as non-negative cell volumes, mesh convexness, geometrically closed cells and boundaries are assumed to be satisfied by having an initially valid mesh and a motion algorithm preserving these criteria. Other measures such as the efficiency, computational cost and robustness (defined as the level of the required user input) are other benchmarks for selecting the appropriate mesh motion algorithm.

There exist different algorithms to handle the motion of the mesh interior points due to the boundaries' movement. Harlow and Welch [110] used the marker and cell method to tackle the problem of moving incompressible flows using N-S equations. Lai and Peskin [113] used immersed boundary (IB) method to investigate moving cylinder simulations. In this method, a region is considered around the moving boundary, moving on a stationary Cartesian grid. The implementation of this method is not trivial and difficulty arises in capturing boundary layer and meeting the mass and momentum conservation [114]. Chesshire and Henshaw [115] developed a composite overlapping grid, consisting of a set of curvilinear component grids, where the continuity condition was imposed at the overlapping boundaries, for the simulation of moving problems. 
In the mesh deformation methods, a mesh motion solver is used to relocate the computational domain internal points according to the prescribed boundary motion. The main challenge in the deforming mesh methods is the preservation of the mesh validity. Indeed, the initial computational mesh has to be valid for the simulations. The motion solver has to maintain the initial mesh validity and the quality measures, mostly referred to the mesh face and cell convexness, orthogonality and skewness [107] during simulations. Therefore the choice of an appropriate mesh motion solver is critical in the moving mesh problems.

The mesh deformation algorithms can be point-based, face-based and/or cell-based. One of the most utilized methods is the point-based spring analogy [116]. In this method, the point-to-point connections, edges, are modeled as elastic linear springs [107] and the point motion is calculated based on the spring response to a moving boundary. The analogy is applied to both structured and unstructured moving mesh applications; yet, it lacks robustness specifically for unstructured finite volume moving mesh simulations [117]. For example, the use of the spring analogy on polyhedral cells may result in the failure of cell topology and quality. In the case of a triangular cell, the corner points on one edge may coincide or move such that cell flipping occurs [107]. These problems could be remedied using nonlinear and torsional springs [118] and [119]. Consequently, having nonlinear and torsional springs add to the degree of complexity of the obtained system of equations, also demanding higher computational time and resources.

The moving solid/flexible boundaries in a mesh cause the deformation of the interior points. The whole deforming computational domain may be modelled as a solid geometry with internal stresses [120]; however, the solution of the obtained stress-strain matrix using existing numerical methods is computationally expensive, due to its highly nonlinear nature.

Therefore, appropriate and fairly inexpensive mesh motion solvers are needed, while keeping the mesh validity and quality [107]. Laplacian smoothing with constant or variable diffusivity is one of the techniques used in Arbitrary Lagrangian-Eulerian (ALE) codes [107]. For example, Littlefield [121] used Laplacian smoothing mesh motion algorithm to determine 
the position of the nodes in hypervelocity impact and penetration, and obtained very accurate results with high mesh quality. Löhner and Yang [122] used Laplacian smoothing on mesh velocities along with variable diffusivity for the mesh points. The variable diffusivity has the advantage of enforcing a more uniform mesh velocity in the near vicinity of the moving boundary, where the most important fluid phenomena and the highest changes in the fluid properties occur. They could reduce the mesh distortion in the close proximity of the moving object, the region that contains small elements. The Laplace smoothing is implemented in OpenFOAM ${ }^{\circledR}[107]$ and is governed by the following equation:

$$
\nabla \cdot(\gamma \nabla u)=0
$$

where $\gamma$ is the diffusion coefficient, which can be constant or variable, and $u$ is the point motion velocity field used to update the point positions based on:

$$
x_{n e w}=x_{\text {old }}+u \Delta t
$$

where $x_{n e w}$ and $x_{\text {old }}$ are the new and old point positions and $\Delta t$ is the time step. In OpenFOAM ${ }^{\circledR}$, Equation 3.29 is discretized based on a second order FEM, where arbitrary polyhedral cells are converted into tetrahedral cells, producing a diagonally equal matrix [107]. The obtained matrix, after applying the boundary conditions, can be treated with PCCG solvers with IC preconditioning [104].

Use of biharmonic equations as the mesh motion solver is another technique used in the moving problems. The advantage of this method is that two conditions on the moving boundary, mesh position and normal mesh spacing, can be specified using a fourth order PDE developed from the second order Laplacian equation [123]. This method is highly useful in free surface and interfacial flow simulations with the main disadvantage of the increased computational time. The biharmonic method is also useful in resolving the boundary layer, but the required accuracy can be obtained in the computationally cheaper Laplacian smoothing 
method using a variable diffusivity [123]. Moreover, according to the Helenbrook [123] the biharmonic method is not optimal for all moving problems, e.g. using mutligrid in solving the system of equations, and Laplacian method is almost computationally cheaper than the biharmonic technique. Johnson and Tezduyar [124] developed a method in which a computational nodes' motion is governed by the linear elasticity equations, the so-called pseudo-solid equations. Indeed, they used the Jacobian matrix obtained from the conversion of physical domain into ALE FEM computational domain, giving the smaller elements a higher rigidity; hence lower element deformation occurs in the close proximity of the moving boundary. However, the solution of the added linear elasticity equations adds to the computational expenses. The mesh deformation is continued until a specified tolerance for linear elasticity equations is achieved and then remeshing is done. It should be mentioned that remeshing projection errors from the old mesh to the new one, the cost of calling an automatic mesh generator at each time, and the added problems in parallel simulations can be counted as the drawbacks of this method, despite the fact that Johnson and Tezduyar [124] proposed some remedies to lower these penalties. Other researchers such as Chiandussi et al. [125] minimized the deformation and distortion of the elements in the pseudo-solid approach and reduced the number of remeshing steps in fluid-structure interaction problems. Dwight [126] modified the pseudo-solid method to account for large boundary translational and rotational motions, the so-called solid body rotation (SBR) stress equation.

Other schemes such as the Transfinite Interpolation between the boundary motion and mesh interior points [127] have also been used in moving structured grid simulations, but they are are unsuitable for unstructured grids [128]. In the case of severe large translational and rotational displacements of unstructured meshes, other mesh deformation methods such as the Radial Basis Function (RBF) interpolation schemes may also be utilized. In this method, the displacements of the boundary nodes are interpolated to the whole computational domain nodes using RBFs [128]. The information about grid connectivity is not needed in this approach and only the system of equations on the boundary nodes is solved; however, 
the performance is strongly dependent on the utilized RBF. Potsdam and Guruswamy [129] used RBFs in multi-block simulations and concluded that the application of this method to all the mesh points in the computational domain is computationally expensive. Other researchers [130] and [114] have done some modifications to increase the efficiency of RBF method.

The number and connectivity of the domain cells are not changed in the deforming mesh algorithms; spring analogy [116], Laplace smoothing [122], Pseudo-solid [124], biharmonic equations [123] and RBF [128] methods. For problems in which large mesh deformations occur, topological changes are useful, where the mesh size and point/face/cell connectivity is varied during the simulation. The rotations of an axial compressor rotor stage is one example for which mesh deformation methods would fail due to the high discretization errors. All possible topological changes are obtained using nine fundamental actions: addition, removal and modification of points, faces and cells at each time step [107].

In the present study, some mesh modifiers, which are obtained by combining the mentioned fundamental topological actions are used, combined and developed to obtain the desired motion. The basic mesh modifiers used are implemented in OpenFOAM ${ }^{\circledR}[131]$ : cell layer addition/removal, sliding interfaces and Generalized Grid Interfaces [132]. Cell layer addition/removal is governed by its triggering mechanism, the specified maximum and minimum layer thicknesses. If the maximum thickness is achieved, a layer is added to the back of the moving object defined bounding box, and when the minimum thickness is achieved layer removal occurs at the front [131]. Sliding interfaces are defined as two detached surfaces which are in relative rotational motion, and are attached at the overlapping region. When the two interfaces rotate and the position of the interfacial faces are changed, the method replaces the faces with new facets to re-achieve one-by-one facet connectivity [131]. Beudoin and Jasak [132] have developed another method, Generalized Grid Interfaces (GGI), to eliminate the need for topological actions performed in Sliding Interface method. Instead, a set of weighting factors is evaluated to balance the flux at the interface [132]. GGI method 
provides a robust tool for large rotational motions such as airfoils' high angle pitching oscillations and turbomachinery applications such as pumps and compressors. GGI is based on the FVM discretization reasoning where the flow properties are transmitted between a pair of conformal/nonconformal patches using weighted interpolation. The so-called master/shadow patches are used for each of the interfaces. A flow variable $\phi$ is obtained using [132]:

$$
\begin{aligned}
\phi_{S i} & =\sum_{n} W_{M n-t o-S i} \phi_{M n}, \\
\phi_{M j} & =\sum_{m} W_{S m-t o-M j} \phi_{S m},
\end{aligned}
$$

where $\phi_{S}$ and $\phi_{M}$ are the flow variables at the shadow and master patches, $i$ and $j$ are the $i$ th and $j$ th shadow and master patch faces, respectively, $n / m$ is the number of master/shadow face neighbors for shadow patch $i / j$, respectively, and $W_{M-t o-S}$ and $W_{S-t o-M}$ show the master facet to shadow facets/shadow facet to master facets weighting factors, respectively.

The calculation of the weighting factors is associated with three constraints as the following:

$$
\begin{aligned}
& \sum_{n} W_{M n-t o-S i}=1 ; \sum_{m} W_{S m-t o-M j}=1, \\
& W_{M n-t o-S i} *\left|S_{M n}\right|=W_{S m-t o-M j} *\left|S_{S m}\right|=\left|S_{\cap_{M-t o-S}}\right|,
\end{aligned}
$$

where $\left|S_{M_{n}}\right|$ is the surface area of master facet, $\left|S_{S_{m}}\right|$ is the surface area of shadow facet and $\left|S_{\cap_{M-t o-S}}\right|$ is the intersection surface area between shadow and master facets. GGI uses several algorithms, such as Sutherland-Hodgman [132] to calculate the weighting factors or Axis Aligned Bounding Box (AABB) to quickly reject the non-overlapping facets. The de- 
scription of these algorithms is beyond the scope of this document and the interested reader is referred to Beudoin and Jasak [132] and the references cited therein.

Different mesh motion algorithms are used and modified in this study to study the unsteady motion of airfoils in single and paired arrangements. Laplacian smoothing [107] is used where the mesh topology is fixed, and dynamic mesh layering and GGI are used, adapted and modified for the paired airfoils simulations. The adopted and developed mesh motion methodologies are applied towards simulations of single and multi airfoil arrangements under different unsteady conditions. The respective results and conducted parametric studies are presented in the subsequent chapters. 


\section{Chapter 4}

\section{On the LRN flow characteristics of a pitching airfoil}

In this chapter, the flow field of a NACA0012 airfoil under harmonic pitching oscillations is investigated using FVM based on 2-D time-dependent, incompressible N-S equations (Equations 3.7) at LRN regime. NACA0012 is chosen due to the availability of relevant studies in the literature for validation purposes. The influence of different unsteady flow and system parameters, namely amplitude of oscillations, reduced frequency and Reynolds number on the lift and drag signatures and the vortical patterns surrounding the airfoil are investigated. The resulting forces are also compared with Theodorsen's method and the numerical database available in the literature. The simulation results reveal that the investigated parameters are of great importance in the aerodynamic performance of the system, as they affect the maximum lift coefficients, hysteresis loops, strength and number of the generated vortices within the harmonic motion, and the extent of the so-called figure-of-eight phenomenon region. Thus, achieving the optimum lift coefficients demands a careful selection of these parameters. 


\subsection{Introduction}

2-D pitching oscillation is one of the prevalent unsteady conditions in LRN flows for which each of the LRN phenomena, such as dynamic stall [1], could occur and affect the flow field noticeably. The aerodynamic performance of LRN pitching airfoils is a function of their instantaneous forces and moments, where these performance benchmarks significantly vary with respect to governing conditions. 2-D LRN studies shed light on the respective flow characteristics and reveal the dependence of the aerodynamic performance on different parameters such as the amplitude of oscillation, reduced frequency, Re and kinematic patterns. These investigations are also of great importance in revealing the effectiveness of the 3-D aspects, such as tip vortices, spanwise flow and vortex interactions. Several studies are conducted to explore the effects of the governing flow and system parameters on the pitching airfoils and wings. The experiments of Jung and Park [39] and Fuchiwaki and Tanaka [40] showed that reduced frequency $(k)$ affects a pitching airfoils' vortex shedding and scaling significantly, with consequent changes in the unsteady dynamic lift [41], drag and moments. Koochesfahani [42] conducted a series of LRN experiments and showed the strong dependence of the resulting wake structures and thrust coefficients of a pitching airfoil on the governing frequency and amplitude of oscillations.

The Euler equation-based simulations of Jameson [66] showed the importance of amplitude of oscillation and effect of ignoring viscous terms in LRN pitching airfoils. Akbari and Price [68] simulated the LRN flow field around a pitching airfoil using N-S equations. They examined the effects of Re, $k$, mean angle of attack $\left(\alpha_{0}\right)$ and pitching axis location $(x / c)$ on the predicted aerodynamic forces and concluded that $k$ and Re have the highest and lowest effects, respectively. Yang et al. [67] examined the impact of $\alpha_{0}$ on the flow field by simulating compressible Euler equation based on FVM. Okongo and Knight [69] simulated 2-D, laminar, compressible flow using N-S equations with FVM and concluded that the main influence of increasing Re is to reduce the angle of attack at which the primary recirculation region starts to develop on the leeward side of the airfoil. They also showed that increasing 
Re causes the location of these recirculation regions to become closer to the LE of the model. Young and Lai [71] investigated the effects of frequency and amplitude of oscillation on the 2-D flow field of a pitching airfoil, where Young and Lai [71] showed that thrust coefficients are dependent on the product $k \alpha$ rather than $k$ or $\alpha$ individually. The 2-D simulations of Visbal and Shang [72] over a pitching airfoil showed that the forces and induced vortical structures depend on the pitch rate and $x / c$. They also showed that the highly unsteady flow structures are accompanied by reverse flow in the boundary layer, shedding of TEV into downstream, and formation of LE vortex with a shear layer on the leeward section of the airfoil.

In this chapter, the flow field of a NACA0012 airfoil is simulated and the effects of different unsteady flow and system parameters on the dynamic forces and vortical patterns are explored in detail, hence a better understanding of the effects of these parameters on corresponding aerodynamic behavior is obtained. These parameters are amplitude of pitching oscillation $(d), k$ and Reynolds number and the study is conducted for the Re range of $555 \leq \mathrm{Re} \leq 5000$. 2-D N-S equations discretized based on FVM using OpenFOAM ${ }^{\circledR}$ are the governing equations and the flow is assumed to be incompressible and laminar. This section will be followed by the numerical simulation method in Section 4.2. Next, results and discussion will be presented including the validation and the conducted parametric studies. Finally, the summary and main conclusions of the chapter will be presented in Section 4.4.

\subsection{Theory and numerical simulations}

\subsubsection{Governing equations and flow solver}

The governing equations for the laminar incompressible flow of a harmonically pitching NACA0012 airfoil are the 2-D N-S equations. These equations are discretized based on FVM in OpenFOAM ${ }^{\circledR}$ and the desired pitching motion is implemented within the code. Diffusive terms are considered due to their importance in LRN flows. The flow is assumed 
to be laminar and incompressible. The governing equations, which consist of continuity and momentum, are solved in a fixed reference frame, and Laplace smoothing mesh motion algorithm [107] is used to perform the mesh motion. In the simulations presented throughout this thesis, convective and diffusive terms are discretized based on a second order central differencing scheme, and the resulting linear system of equations is treated with a Preconditioned Conjugate Gradient (PCG) solver. The pressure and the pressure-velocity coupling was handled using a Pressure Implicit and Splitting of Operators (PISO) algorithm. , and the first order Euler implicit scheme is used for the transient terms

\subsubsection{Mesh generation and boundary conditions}

An O-type mesh is generated to model the airfoil and the surrounding flow. The selection of the O-type mesh over a C-type is based on an earlier study by Yang et al. [67]. They showed that O-type mesh yields almost the same aerodynamic forces as those resulting from C-type, but needs much less computer time. The schematic of the O-type grid used in the present simulations is shown in Fig. 4.1.

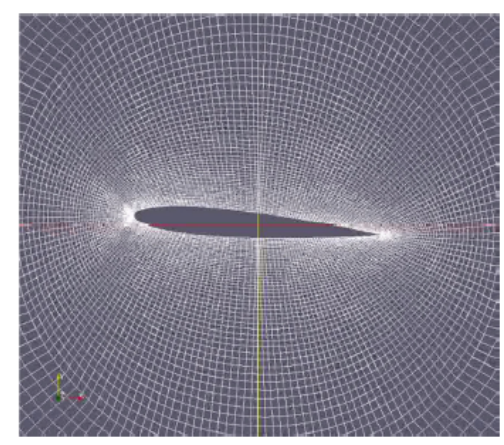

Figure 4.1: Schematic of the O-type grid around the airfoil.

The continuity equation demands initial conditions on $p$, while momentum equations need the initial velocity components on $u$ and $v$. The steady state solutions are used as the initial conditions for the time-marching calculations. Along the airfoil surface a noslip boundary condition is applied. The far-field boundary is set to $30 \mathrm{c}$ from the airfoil to minimize its undesired effects on the flow surrounding the moving surface $[80,88]$ and is set 
to a slip boundary condition, as illustrated in Fig. 4.2. At the inflow boundary, the velocity is specified (velocity Dirichlet boundary condition) based on the desired Re, and the pressure is restricted to the zero-gradient condition. At the outflow boundary, the pressure is set to the freestream value, while the velocity is set to the zero-gradient condition. The present simulations are conducted for a mesh size of $26 \times 10^{3}$ and temporal resolution of 2000 time steps within one oscillation cycle.

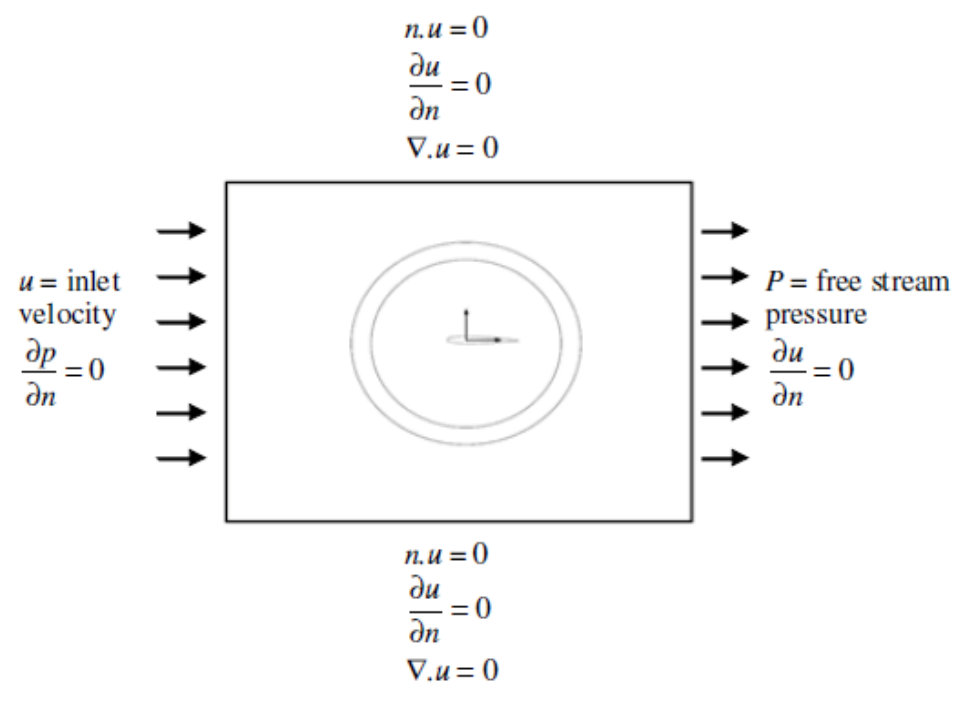

Figure 4.2: Schematic of the O-type grid around the airfoil.

\subsubsection{Validation studies}

In order to assess the accuracy of the results, extensive numerical tests are performed to address the issues concerning grid sensitivity and time step resolution. Also, several simulations are conducted and the results are compared with the database available in the literature ( [68], [133] and [134]). The lift coefficients are also compared with those obtained from Theodorsen's method. This is done to show that Theodorsen's method [8] only yields reasonable results when the effect of viscosity is minimal, and that the numerical simulations greatly differ from Theodorsen's results both qualitatively and quantitatively, when 
significant viscosity and vortical structures exist. Since the quality of the simulation results depends on the mesh and temporal resolution, a convergence study is first carried out to find the appropriate mesh size and time step for the present study. The pitching motion equation for the mesh dependence analysis is $\alpha=\alpha_{0}+d \sin (2 \pi f t)$. This grid analysis is performed for several cases; however, only the results for $d=2^{\circ}, \operatorname{Re}=555$ and $d=10^{\circ}$, $\mathrm{Re}=5000$ are presented here for the sake of brevity. Four different mesh sizes were considered: $5 \times 10^{3}, 11 \times 10^{3}, 26 \times 10^{3}$ and $50 \times 10^{3}$, corresponding to $100,140,176$, and 200 points on the airfoil surface, respectively, and each simulation emerged from its fully converged stationary solution. The instantaneous lift coefficients $\left(C_{l}\right)$ versus time were obtained for each case, and since they are small, the close view of their peak values is depicted in Figs. 4.3(a) and 4.3(b). The difference between the $C_{l}$ values computed from $26 \times 10^{3}$-cell and $50 \times 10^{3}$-cell models was found to be negligible. Thus, the mesh of $26 \times 10^{3}$ cells was selected as the baseline mesh for further analyses.

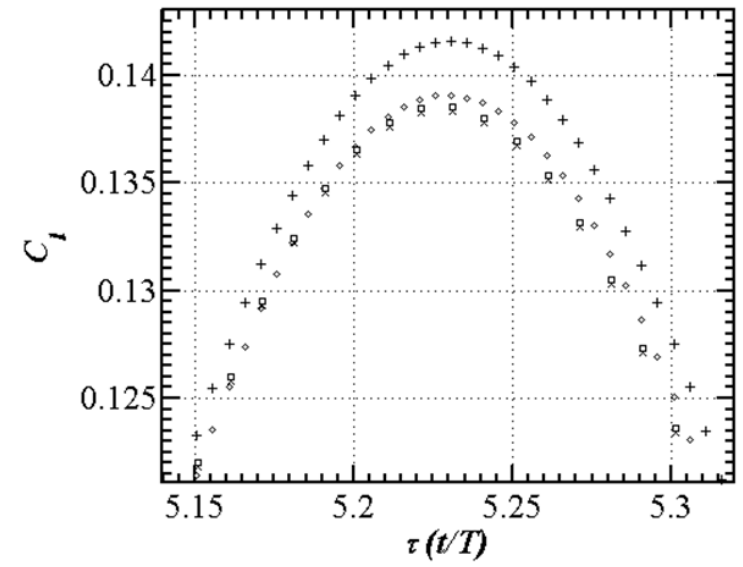

(a)

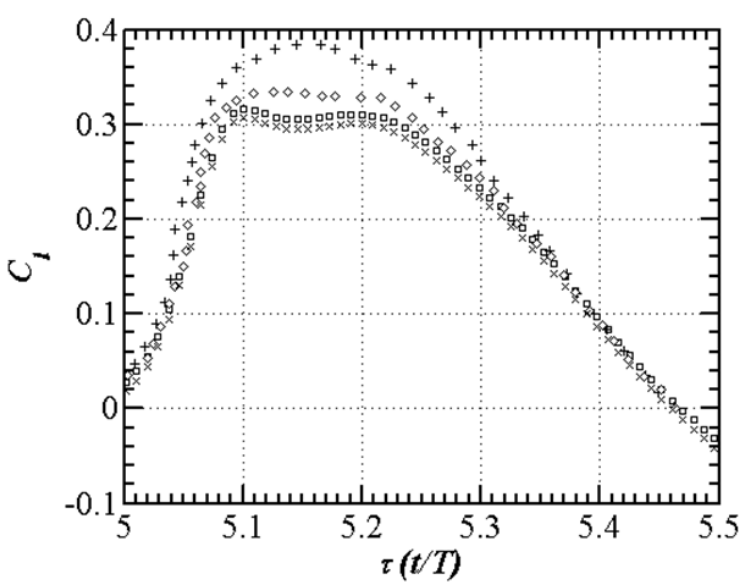

(b)

Figure 4.3: Instantaneous peak lift coefficients $\left(C_{l}\right)$ versus nondimensional time $(\tau)$ for different mesh sizes $(k=0.1, x / c=0.25):+, 5 \times 10^{3} ; \diamond, 11 \times 10^{3} ; \square, 26 \times 10^{3} ; \times, 50 \times 10^{3}$, (a) $d$ $=2^{\circ}$ and $\operatorname{Re}=555 ;(\mathrm{b}): d=10^{\circ}$ and $\operatorname{Re}=5000$.

Similar observations are also made for the temporal resolution analysis. The baseline grid $\left(26 \times 10^{3}\right)$ was examined for several time steps: 500, 1000, 2000 and 4000, within one excitation period. As expected [104], the results are less sensitive to the temporal resolution 
than the spatial refinement. The lift coefficients for 2000 and 4000 time steps had negligible differences, Table 4.1. Therefore, with the present solver settings, $26 \times 10^{3}$ cells and 2000 time steps in a pitching cycle are sufficient and yield accurate results for the dynamic pitching simulations.

Table 4.1: Spatial and temporal sensitivity analyses, difference is calculated based on the lift coefficient of the base line case $\left(26 \times 10^{3}\right.$ cells and 2000 time steps in a cycle).

\begin{tabular}{ccc}
\hline \hline $\mathrm{T} / \Delta t$ & Grid Size $\times 10^{3}$ & Difference $(\%)$ \\
\hline 500 & 26 & 1.47 \\
1000 & 26 & 1 \\
4000 & 26 & 0.2 \\
2000 & 5 & 2.6 \\
2000 & 11 & 1.2 \\
2000 & 50 & 0.4 \\
\hline
\end{tabular}

The simulations are further validated against the existing literature. First, the flow field of a pitching circular cylinder is simulated following the numerical studies by Mahfouz and Badr [133] and Okajima et al. [134]. The nondimensional surface velocity of the cylinder is given by $U_{w}=0.2 \sin (0.1 \pi t)$ and $\operatorname{Re}=40$. The calculated $C_{l}$ is in good agreement with that of Mahfouz and Badr [133] and Okajima et al. [134], as shown in Fig. 4.4.

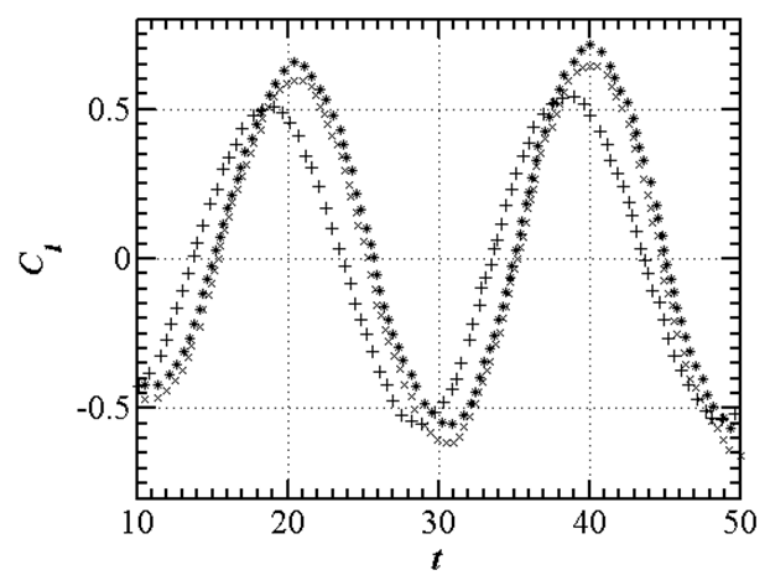

Figure 4.4: Instantaneous lift coefficient of a pitching cylinder at $\operatorname{Re}=40, U_{w}=0.2$ $\sin (0.1 \pi t)$ : +, Mahfouz and Badr [133]; × Okajima et al. [134]; *, present simulation.

Moreover, the pitching oscillation of a NACA0012 airfoil studied by Akbari and Price [68] 
is investigated. The pitching oscillation is governed by $\alpha=15^{\circ}+10^{\circ} \cos (2 \pi f t)$, and $k=$ $0.25\left(k=\frac{\pi f c}{U_{\infty}}\right)$ and $\operatorname{Re}=3000$. Fig. 4.5 shows the normal force coefficient $\left(C_{n}\right)$ versus $\alpha$, which is in close agreement with that of Akbari and Price [68]. The observed discrepancy could be related to the different LE/TE shapes considered for the NACA0012 models (see Akbari and Price [68]), or it could be due to the different utilized time and grid resolutions between the present study and that of Akbari and Price [68].

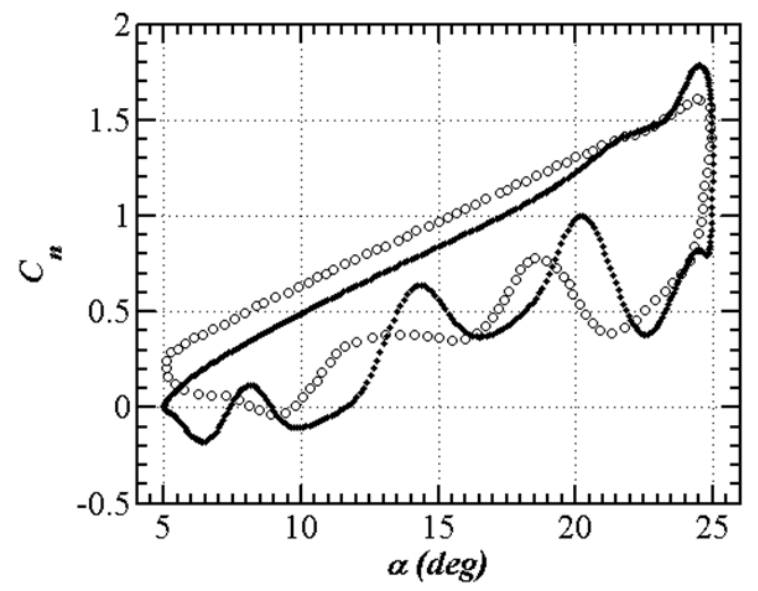

Figure 4.5: Normal force coefficient $\left(C_{n}\right)$ of a pitching NACA0012 at $\operatorname{Re}=3000\left(\alpha=15^{\circ}\right.$ $\left.+10^{\circ} \cos (2 \pi f t), k=0.25, x / c=0.25\right)$ : -, present simulation; o, Akbari and Price [68].

Finally, the lift coefficients obtained from this numerical study are compared with those calculated from Theodorsen's method. The details of Theodorsen's method are well documented (see, e.g., Leishman [8] and the references cited therein); however, for the sake of completeness, a brief explanation is given here.

Theodorsen's method for pitching airfoils is based on inviscid, incompressible and small disturbance assumptions and yields the aerodynamic lift by the following equation:

$$
L=\pi \rho V^{2} b\left[\frac{b}{V} \dot{\alpha}-\frac{b^{2}}{V^{2}} a \ddot{\alpha}\right]+2 \pi \rho V^{2} b\left[\alpha+\frac{b \dot{\alpha}}{V}\left(\frac{1}{2}-a\right)\right] C(k),
$$

where $\rho, b, V, \alpha, a$ and $C(k)$ are respectively the density, airfoil half-chord length, flow velocity, instantaneous angle of attack, pitching axis location and Theodorsen's function. 
For pure pitching oscillations $\left(\alpha=\bar{\alpha} e^{i \omega t}\right)$ the lift coefficient $\left(C_{l}\right)$ is obtained by the following equation:

$$
C_{l}=2 \pi(F[1+i k]+G[i-k]) \bar{\alpha} e^{i \omega t}+\pi k\left(i-\frac{k}{2}\right) \bar{\alpha} e^{i \omega t},
$$

where $k$ is the reduced frequency, and $F$ and $G$ are the real and imaginary parts of Theodorsen's function $(\mathrm{C}(k)=\mathrm{F}(k)+\mathrm{iG}(k))$, respectively. In the present study, the pitching motion is governed by the following equation:

$$
\alpha=\alpha_{0}+d \sin (2 \pi f t)
$$

The simulation is conducted for both viscous and inviscid flows where $\operatorname{Re}=555, k=0.1$ and $d=2^{\circ}$. According to Leishman [8], Theodorsen's method gives accurate results for small amplitudes of oscillation. Figure 4.6 shows that our inviscid simulation matches the results of Theodorsen's method; however, for the viscous flow case, the numerically computed lift coefficients are lower than those calculated from Theodorsen's method as the simulations are viscous and the theory is inviscid, showing the contribution of viscous terms in the generation of the lift coefficient, Fig. 4.6.

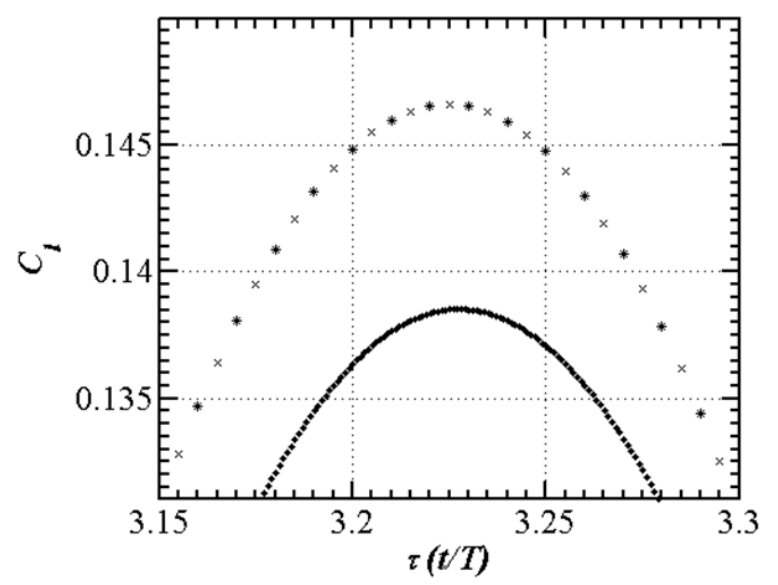

Figure 4.6: Instantaneous lift coefficient versus nondimensional time $(\operatorname{Re}=555, k=0.1, d$ $\left.=2^{\circ}\right)$ : - , viscous NS simulation; $\times$, inviscid N-S simulation; ${ }^{*}$, Theodorsen .

Figure 4.7 shows the lift coefficients versus nondimensional time for $d=10^{\circ}$. In this 
figure, the lift coefficient computed by the numerical solution of the viscous N-S equations is compared with that calculated via Theodorsen's method. Figure 4.7 shows that the difference between the analytical and numerical results is increased for $d=10^{\circ}$, which can be related to the breakdown of Kutta condition [see Leishman [8]] and the stronger effects of vortices around TE, which cause inaccuracies in the predictions of the analytical method.

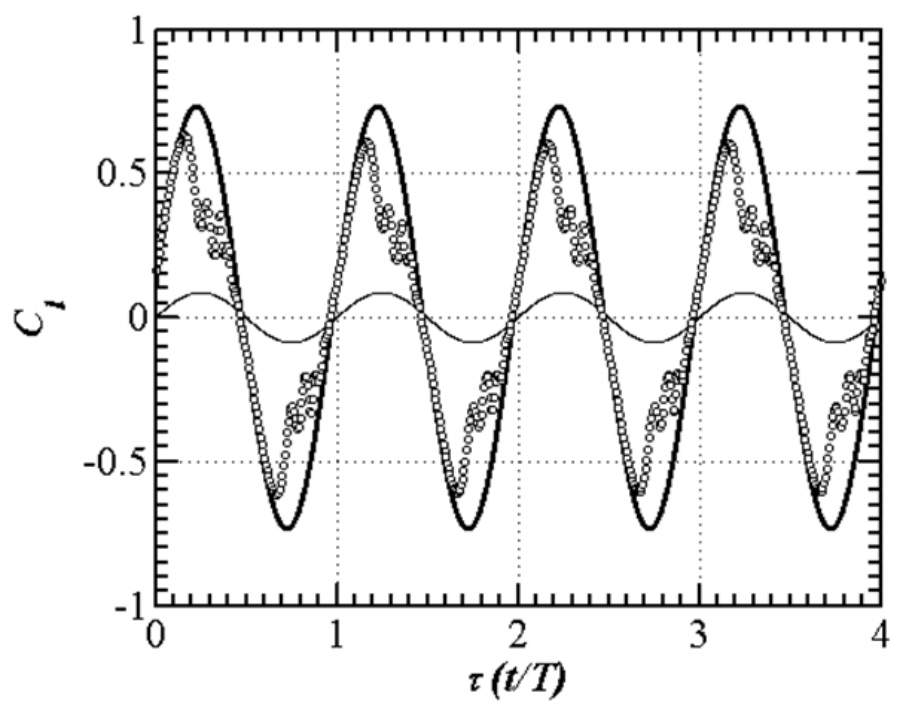

Figure 4.7: Instantaneous lift coefficient versus nondimensional time $(\operatorname{Re}=555, k=0.1, d$ $=10^{\circ}$ ): o, numerical; -, Theodorsen; (Airfoil pitch angle is also shown on the figure as $\alpha / 2$ ).

\subsection{Results and Discussion}

The present parametric study is carried out to investigate the influence of $d, k$ and Re on the aerodynamics of a NACA0012 when $x / c=0.25$ and $\alpha_{0}=0$. A summary of the cases studied is shown in Fig. 4.8. The parametric space corresponds to LRN flows where the taken governing parameters and their combination are not paid a due attention in the literature. Table 4.2 shows the available data in the literature with their studied parametric space.

The emphasis in this work is put on the influence of the above-mentioned parameters on the generated lift and drag coefficients, and wherever necessary the vortical patterns around 
Table 4.2: Summary of experimental and numerical pitching studies in the literature.

\begin{tabular}{|l|l|}
\hline Author & Performed investigation \\
\hline Akbari and Price [68] & 2-D, Numerical (N-S), Re $=3000,10^{4}$, high $\alpha, \alpha_{0}=15^{\circ}, 20^{\circ}$, \\
& effect of Re, $k, \alpha_{0}, x / c$ \\
Hamdani and Sun [70] & 2-D, Numerical (N-S), Re $=100$, high $\alpha$, effect of $k$ \\
Jameson [66] & 2-D, Numerical (Euler), $d=5^{\circ}$ \\
Koochesfahani [42] & 2-D, Experimental, Re $=12000, f=0.5-6 \mathrm{~Hz}, d=2^{\circ}, 4^{\circ}$, \\
& effect of $f$ and $d$ on the wake structures \\
Okongo and Knight [69] & 2-D, Numerical (N-S), Re $=10^{4}, 2 \times 10^{4}$, effect of Re \\
Shih et al. [43] & 2-D, Experimental (PIV), Re $=5000-25000,10^{\circ}<d$ \\
Yang et al. [67] & 2-D, Numerical (Euler), $d=5^{\circ}$, effect of $\alpha_{0}$ \\
Young and Lai [71] & 2-D, Numerical (N-S), Re $=12 \times 10^{4}$ \\
\hline
\end{tabular}

the airfoil are discussed in detail. It should be mentioned that all the presented instantaneous force coefficients are presented for one pitching cycle when the numerical transient terms fade and the results steadily change in each cycle.

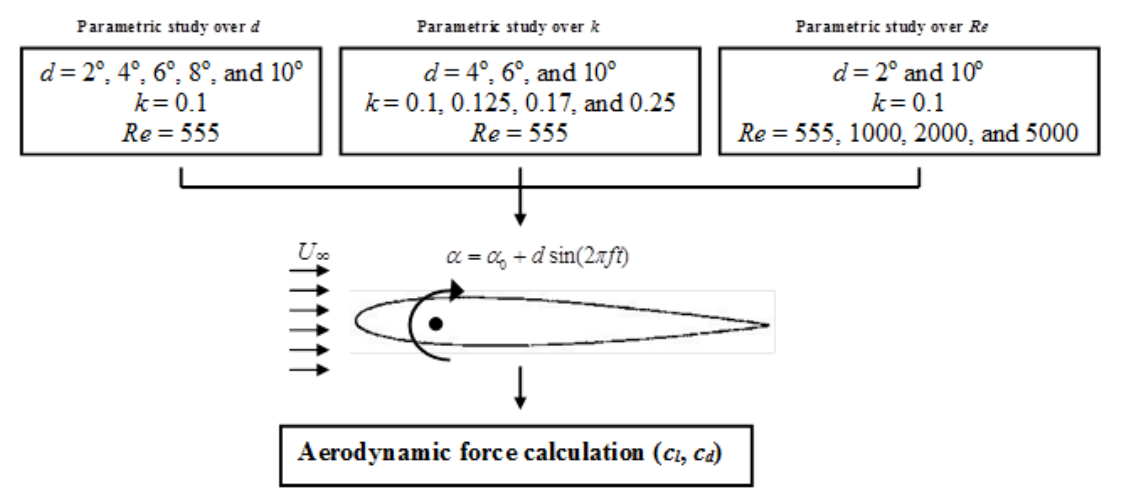

Figure 4.8: Schematic view of the conducted case studies.

\subsubsection{Effects of amplitude of oscillation}

The effect of the airfoil amplitude of oscillation on the simulated lift coefficients is first explored. The instantaneous $C_{l}$ versus $\tau$ is depicted in Fig. 4.9 for $d=2^{\circ}, 4^{\circ}, 6^{\circ}, 8^{\circ}$ and $10^{\circ}$, where $k=0.1$ and $\operatorname{Re}=555$. As illustrated, the maximum lift coefficient $\left(C_{l_{\max }}\right)$ increases at higher amplitudes of oscillation. The calculated lift coefficients are periodic and resemble harmonic-like patterns; however, for $d=8^{\circ}$ there is a deviation from sinusoidal behavior 
after the peak lift coefficient. This deviation is more noticeable in the $d=10^{\circ}$ case, which can be explained by the generation of three consecutive vortices and flow separation at the TE of the airfoil. These counter-rotating vortical structures are shown in Fig. 4.10.

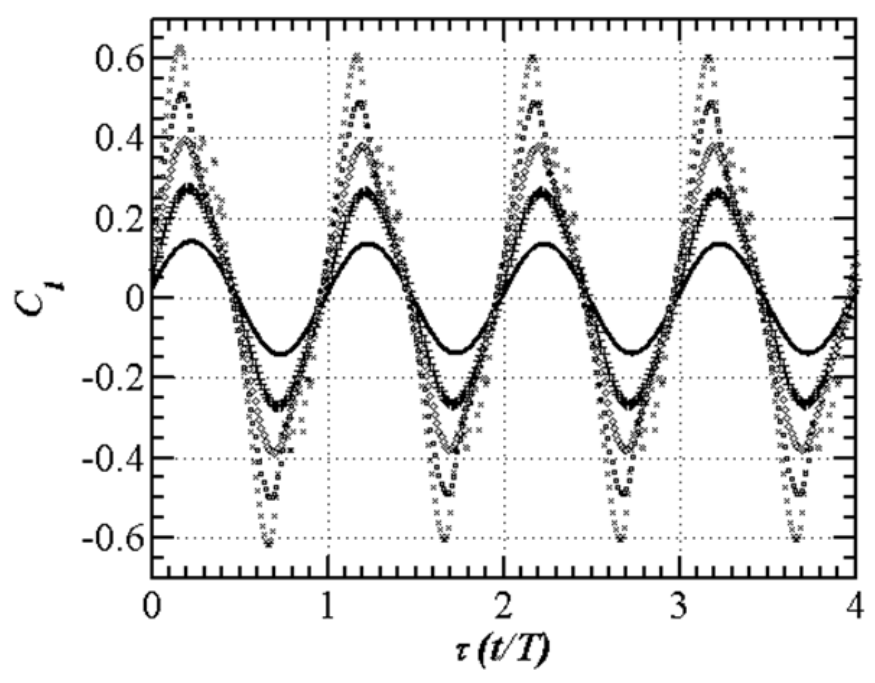

Figure 4.9: Instantaneous lift coefficient $\left(C_{l}\right)$ versus nondimensional time $(\tau):(-), d=2^{\circ}$; $(+), d=4^{\circ} ;(\diamond), d=6^{\circ} ;(\square), d=8^{\circ} ;(\times), d=10^{\circ}$.

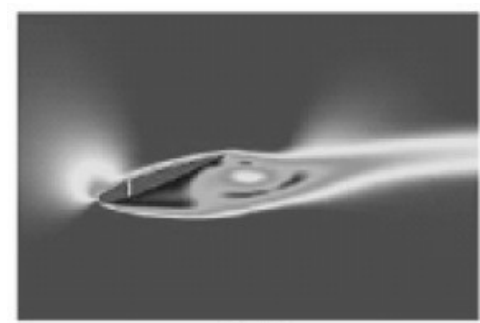

(a)

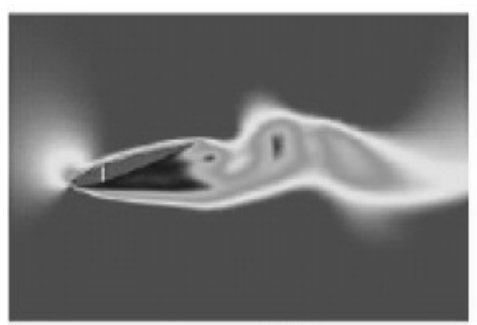

(b)

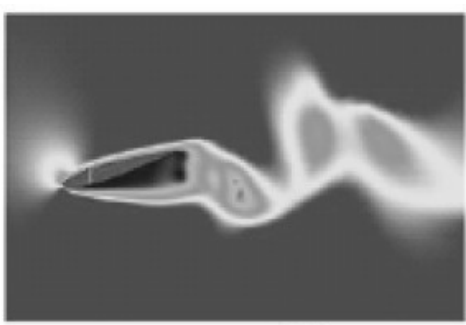

(c)

Figure 4.10: The generated vortical structures after the peak lift coefficient $C_{l_{\max }}, d=10^{\circ}, k$ $=0.1, \operatorname{Re}=555$ : (a), $\alpha=-9.75^{\circ}$; (b), $\alpha=-7.8^{\circ}$; (c), $\alpha=-5.6^{\circ}$, the airfoil is in up-stroke.

Furthermore, increasing $d$ induces significant lead in the $C_{l}$ results meaning that $C_{l_{\max }}$ is obtained at a lower $\tau$ (Fig. 4.9). This can be attributed to the stronger effects of the shed wake and vortical structures on the surrounding fluid at the higher amplitudes. Figure 4.11 shows $C_{l}$ versus $\alpha$ for the conducted simulations. As it can be seen, the hysteresis loops are broadened by increasing the amplitudes. It means that at larger amplitudes, for the same angle of attack, the airfoil produces higher lift during downstroke than the upstroke. 
The existence of these loops is the result of induced velocities, which result in different lift coefficients between the up and downstrokes.

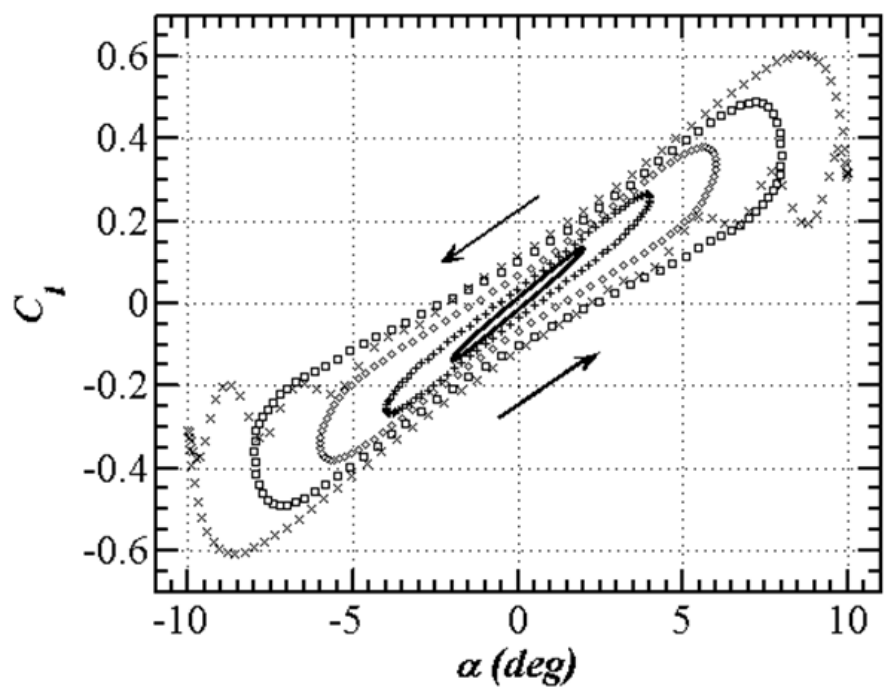

Figure 4.11: Lift coefficient $\left(C_{l}\right)$ versus angle of attack $(\alpha), k=0.1, \operatorname{Re}=555:(-), d=2^{\circ}$; $(+), d=4^{\circ} ;(\diamond), d=6^{\circ} ;(\square), d=8^{\circ} ;(\times), d=10^{\circ}$.

The predicted drag coefficients versus angle of attack are illustrated in Fig. 4.12 showing a figure-of-eight-like pattern. Likewise the $C_{l}$ curves (Fig. 4.11$)$, the maximum value $\left(C_{d_{\max }}\right)$ as well as hysteresis-loop width are increased at the higher amplitudes of oscillation. The upstroke $C_{d}$ is higher than the downstroke one, which is contrary to the trend seen in Fig. 4.11. Also, $C_{d_{\min }}$ is almost constant and does not change with $d$ noticeably.

\subsubsection{Effects of reduced frequency}

The effect of reduced frequency on the unsteady pitching motion is investigated by plotting the $C_{l}$ versus $\alpha$ curves for different reduced frequencies: $k=0.1,0.125,0.17$ and 0.25 at $\operatorname{Re}$ $=555$ and $d=4^{\circ}, 6^{\circ}$ and $10^{\circ}$. As shown in Fig. $4.13\left(d=4^{\circ}\right), C_{l_{\max }}$ increases with reduced frequency; from $C_{l_{\max }}=0.265$ at $k=0.1$ to $C_{l_{\max }}=0.27$ at $k=0.125$ and from $C_{l_{\max }}=$ 0.275 at $k=0.17$ to $C_{l_{\max }}=0.28$ at $k=0.25$. Moreover, a higher $k$ broadens the hysteresis loops, but does not have any noticeable effect on the lift curve slope. 


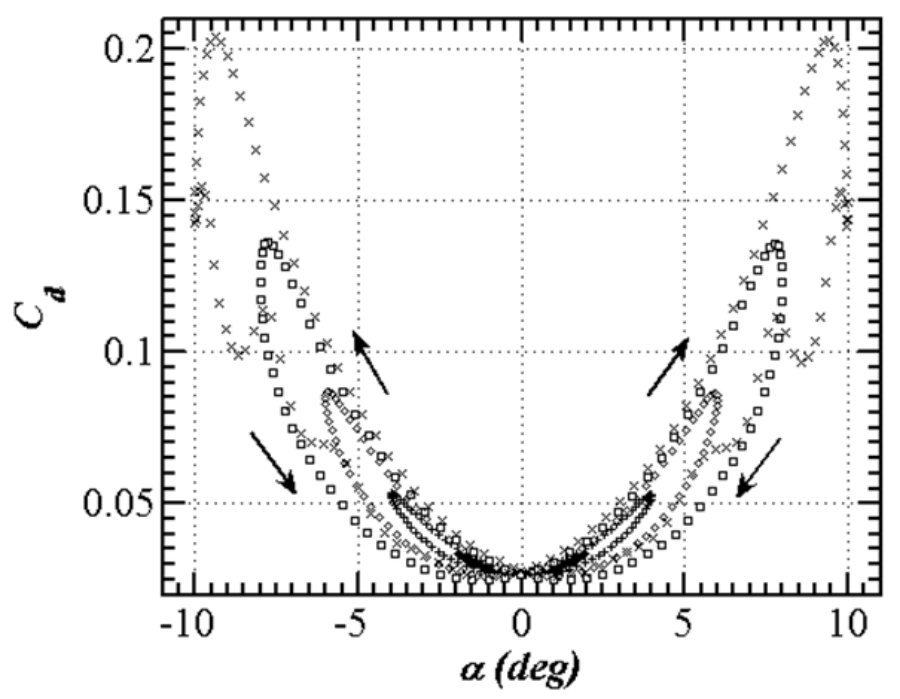

Figure 4.12: Drag coefficient $\left(C_{d}\right)$ versus angle of attack $(\alpha), k=0.1, \operatorname{Re}=555:(-), d=2^{\circ}$; $(+), d=4^{\circ} ;(\diamond), d=6^{\circ} ;(\square), d=8^{\circ} ;(\times), d=10^{\circ}$.

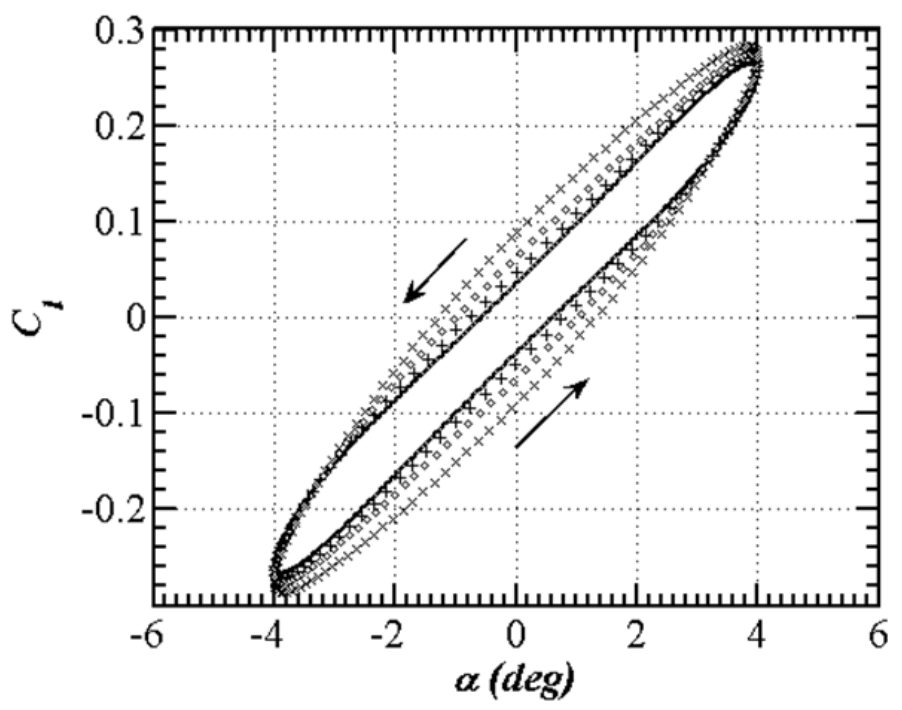

Figure 4.13: Lift coefficient $\left(C_{l}\right)$ versus angle of attack $\left.(\alpha), d=4^{\circ}, \operatorname{Re}=555\right):(-), k=0.1$; $(+), k=0.125 ;(\diamond), k=0.17 ;(\times), k=0.25$.

A similar behavior is observed for $d=6^{\circ}$ (Fig. 4.14); however, in this case, $k$ has a stronger effect on the lift coefficients and a more noticeable effect on $C_{l_{\max }}$. That is, increasing $k$ changes $C_{l_{\max }}$ from 0.38 at $k=0.1$ to $C_{l_{\max }}=0.4$ at $k=0.125$ and from $C_{l_{\max }}$ $=0.41$ at $k=0.17$ to $C_{l_{\max }}=0.43$ at $k=0.25$.

Figure 4.15 reveals that increasing $k$ at $d=10^{\circ}$, where there are consecutive vortical 


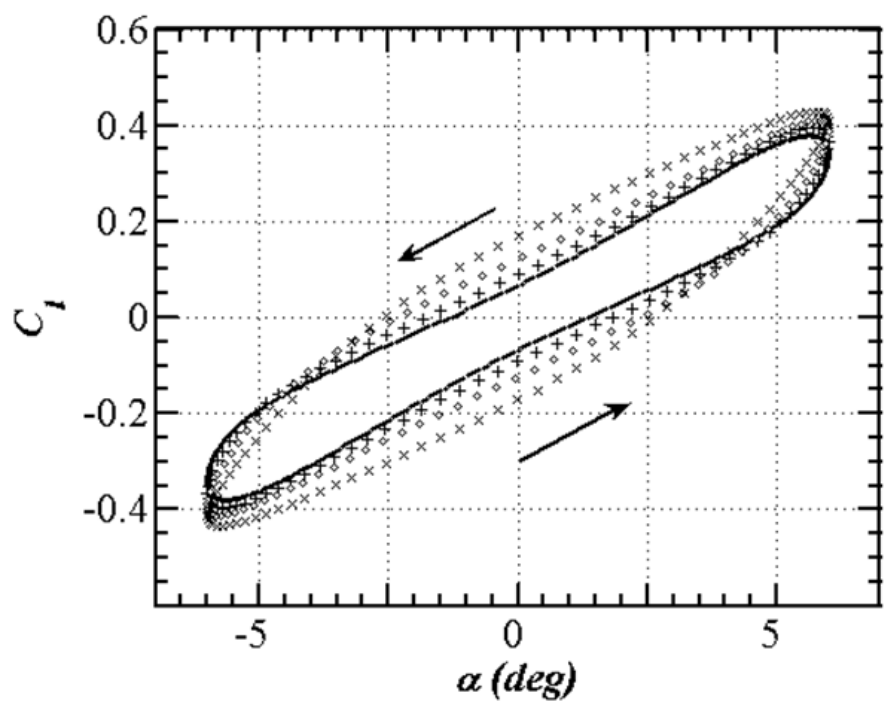

Figure 4.14: Lift coefficient $\left(C_{l}\right)$ versus angle of attack $(\alpha), d=6^{\circ}, \operatorname{Re}=555:(-), k=0.1$; $(+), k=0.125 ;(\diamond), k=0.17 ;(\times), k=0.25$.

patterns in the $C_{l}$ curves, has considerable influence on the strength of these vortices and their initiation location during the harmonic motion. As described in the previous section, three consecutive vortices are generated at TE after the peak value of lift for $k=0.1$ and $d=$ $10^{\circ}$. These vortices amplify the lift force from the instant they are generated to the instant of their separation and shedding into the downstream wake. As $k$ is increased to 0.125 , the pitch angle at which these vortices form is changed from $\alpha=10^{\circ}$ at $k=0.1$ to $\alpha=9.5^{\circ}$ at $k=0.125$. The effect of these two vortices is observed at $k=0.125$ (Fig. 4.15) in the lift coefficient curves, the second one occurring at $\alpha=7^{\circ}$. At $k=0.17$, the first vortex starts to develop and sheds at $\alpha \approx 8^{\circ}$. Moreover, as shown in Fig. 4.15, the number of effective vortices in the lift generation is reduced from three at $k=0.1$ to two at $k=0.125$ and to one at $k=0.17$. The pitch angle of the first vortical structure is further changed to $\alpha \approx 4^{\circ}$ for $k=0.25$, and this vortex is the only effective one in the simulated lift coefficients.

Figure 4.16 shows the respective $C_{d}$ versus $\alpha$ curve of Fig. 4.15. As shown, increasing the reduced frequency increases $C_{d_{\max }}$ and broadens the hysteresis-loops width. The effect of $k$ during the downstroke is greater than during the upstroke. The minimum drag coefficient remains constant for $k=0.1,0.125$ and 0.17 ; however, it is reduced for $k=0.25$, where the 


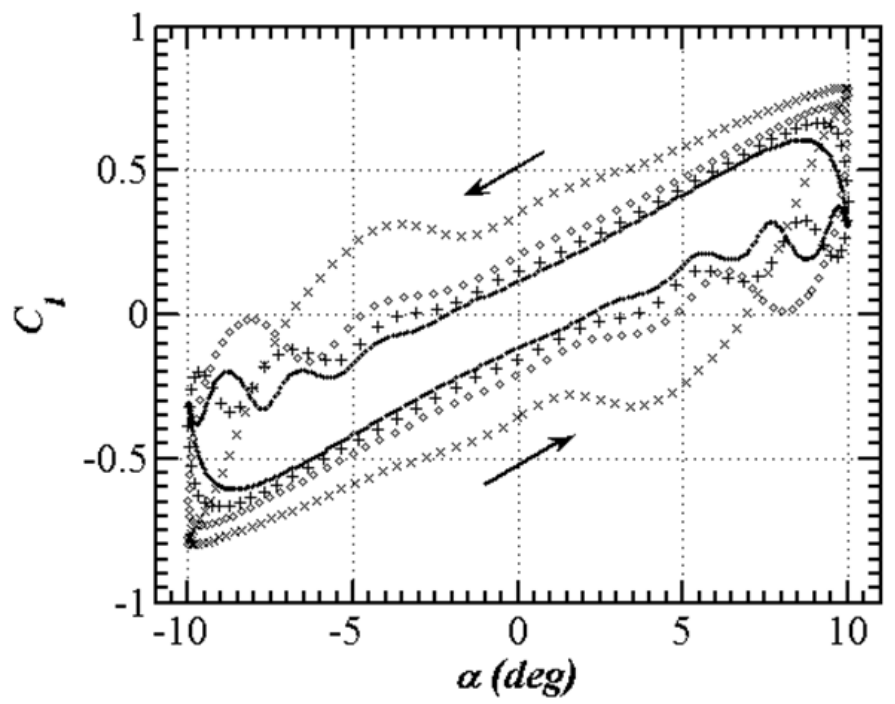

Figure 4.15: Lift coefficient $\left(C_{l}\right)$ versus angle of attack $(\alpha), d=10^{\circ}, \operatorname{Re}=555:(-), k=0.1$; $(+), k=0.125 ;(\diamond), k=0.17 ;(\times), k=0.25$.

number of the vortical structures is reduced to one and occurs at $\alpha \approx 4^{\circ}$.

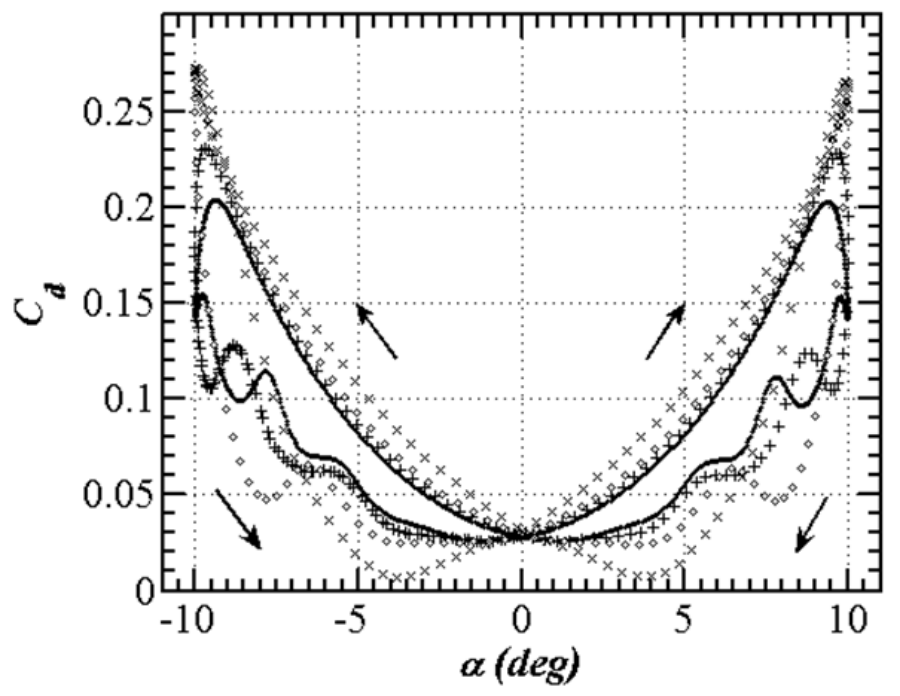

Figure 4.16: Drag coefficient $\left(C_{d}\right)$ versus angle of attack $(\alpha), d=10^{\circ}, \operatorname{Re}=555:(-), k=$ $0.1 ;(+), k=0.125 ;(\diamond), k=0.17 ;(\times), k=0.25$. 


\subsubsection{Effects of Reynolds number}

The influence of Re on the simulated lift coefficients is studied for $\operatorname{Re}=555,1000,2000$ and 5000. This investigation is conducted for $k=0.1$ and $d=2^{\circ}$ and $10^{\circ}$, which are the lowest and highest considered amplitudes of oscillation in this chapter. As Re is increased from Re $=555$ to $\operatorname{Re}=1000$ for $d=10^{\circ}, C_{l_{\max }}$ increases from $C_{l_{\max }}=0.61$ to $C_{l_{\max }}=0.67$ (see Fig. 4.17); however, the pitch angle at which $C_{l_{\max }}$ occurs remains the same, $\alpha \approx 9^{\circ}$ for both $\operatorname{Re}$.

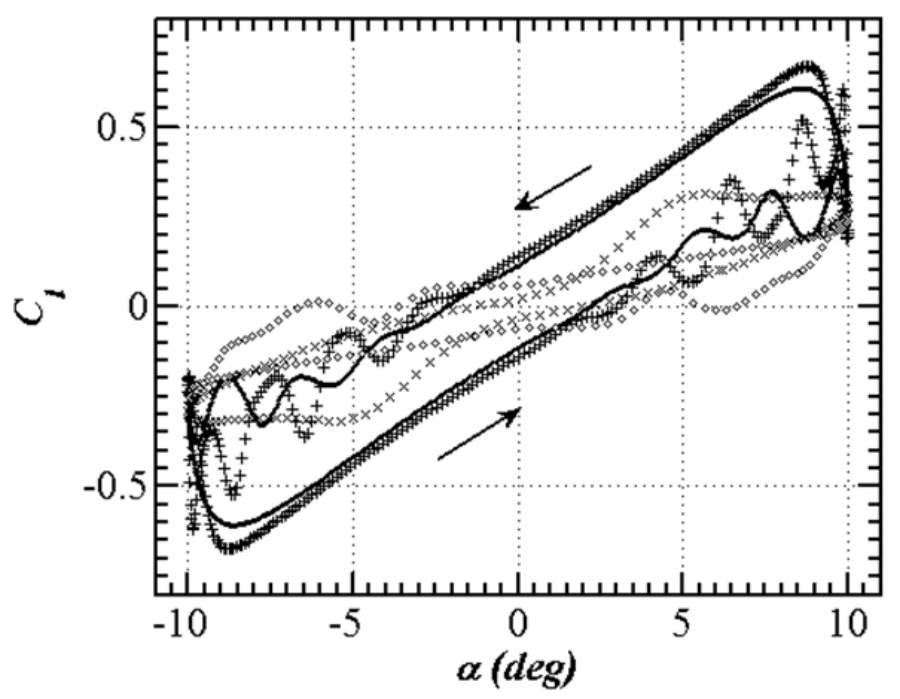

Figure 4.17: Lift coefficient $\left(C_{l}\right)$ versus angle of attack $(\alpha), d=10^{\circ}, k=0.1:(-), \operatorname{Re}=555$; $(+), \operatorname{Re}=1000 ;(\diamond), \operatorname{Re}=2000 ;(\times), \operatorname{Re}=5000$.

The increase in $C_{l_{\max }}$ at $\mathrm{Re}=1000$ is accompanied by a figure-of-eight-like phenomenon at $\alpha \approx 9.5^{\circ}$. After this point, the upstroke lift coefficients are higher than those at the downstroke at the same angle of attack. Both $\mathrm{Re}=555$ and 1000 include three sudden riseand-falls after the peak lift coefficients. This behaviour can be attributed to the generation of extensive vortical patterns after the maximum angle of attack. Further increase in Re, Re $=2000$, results in decrease in $C_{l_{\max }}$ and decrease in the figure-of-eight-like phenomenon region. However, further increase of Re to 5000 slightly increases $C_{l_{\max }}$ and eliminates the figure-of-eight-like phenomenon. Figs. 4.18(a)-(d) show the vortical patterns around the airfoil when $C_{l_{\max }}$ is achieved. 


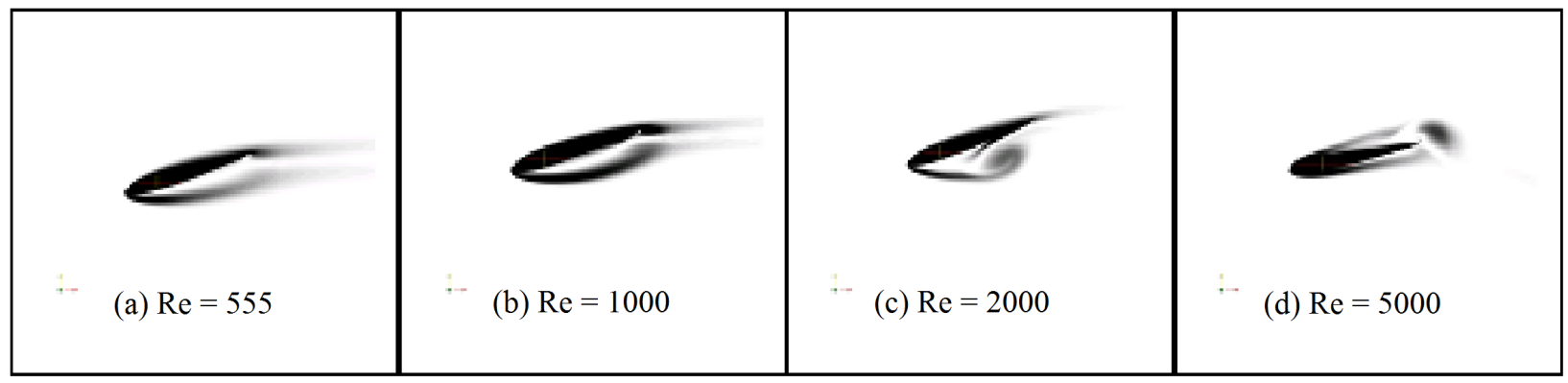

Figure 4.18: Vortical patterns around the airfoil when maximum lift coefficient $\left(C_{l_{\max }}\right)$ is obtained $\left(d=10^{\circ}, k=0.1\right)$.

As can be seen, increasing Re from 555 to 1000 slightly increases the size of the TE vortex and the strength of the lower surface vortex. Further increase of Re from 1000 to 2000 causes the lower surface vortex to separate, which could be the reason for the decrease of $C_{l_{\max }}$ previously observed in Fig. 4.17. The lower and upper surface vortices are not separated from the airfoil at $\mathrm{Re}=5000$, but the TEV is separated and convected into the downstream wake. This could be the reason for higher $C_{l_{\max }}$ at $\mathrm{Re}=5000$ than that $\operatorname{Re}=$ 2000, and the reason for the lower $C_{l_{\max }}$ than those at $\operatorname{Re}=555$ and 1000 . The effect of increasing Re is also investigated at the lowest explored amplitude of oscillation, $d=2^{\circ}$, and the same trends as those effects of Re at $d=10^{\circ}$ are observed (see Fig. 4.19). Figure 4.20 shows the $C_{d}$ versus $\alpha$ curves at $d=10^{\circ}$ for different Reynolds numbers. As can be seen, $C_{d_{\min }}$ is reduced by increasing Re from 555 to 2000 , but increases for $\operatorname{Re}=5000$.

\subsection{Summary and conclusion}

A numerical aerodynamics study was carried out for a 2-D flow around a NACA0012 airfoil performing dynamic pitching motion in LRN regime. The aerodynamic characteristics of the model were explored and the effect of some unsteady flow and system parameters $(d$, $k$ and $\mathrm{Re}$ ) on the instantaneous force coefficients and flow patterns were investigated. The analysis was conducted by means of N-S equations discretized based on FVM. The results show the substantial influence of the aforementioned unsteady parameters on the maximum 


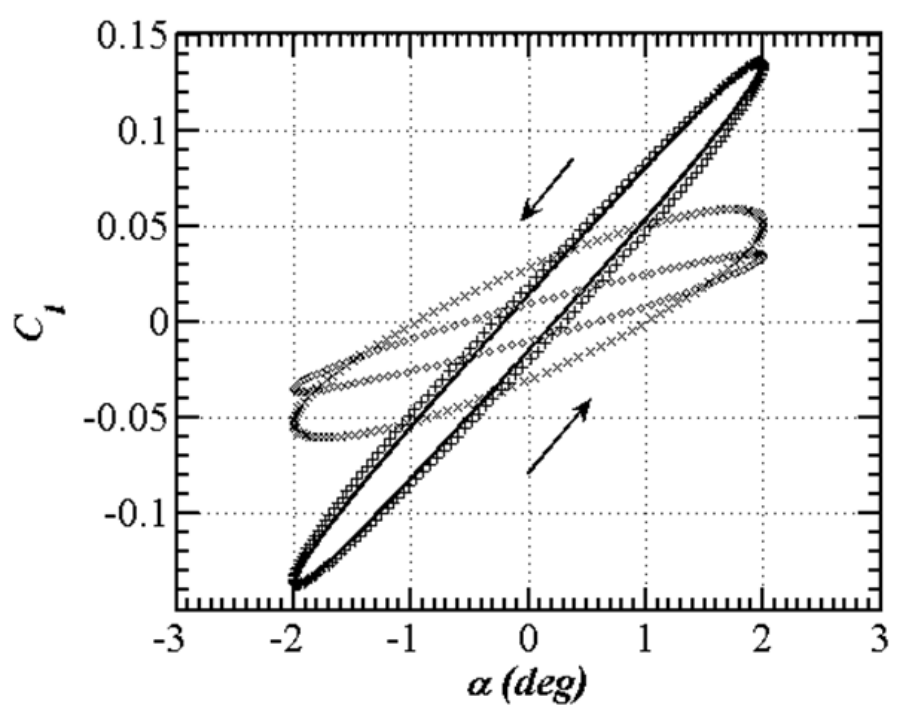

Figure 4.19: Lift coefficient $\left(C_{l}\right)$ versus angle of attack $(\alpha), d=2^{\circ}, k=0.1:(-), \operatorname{Re}=555$; $(+), \operatorname{Re}=1000 ;(\diamond), \operatorname{Re}=2000 ;(\times), \operatorname{Re}=5000$.

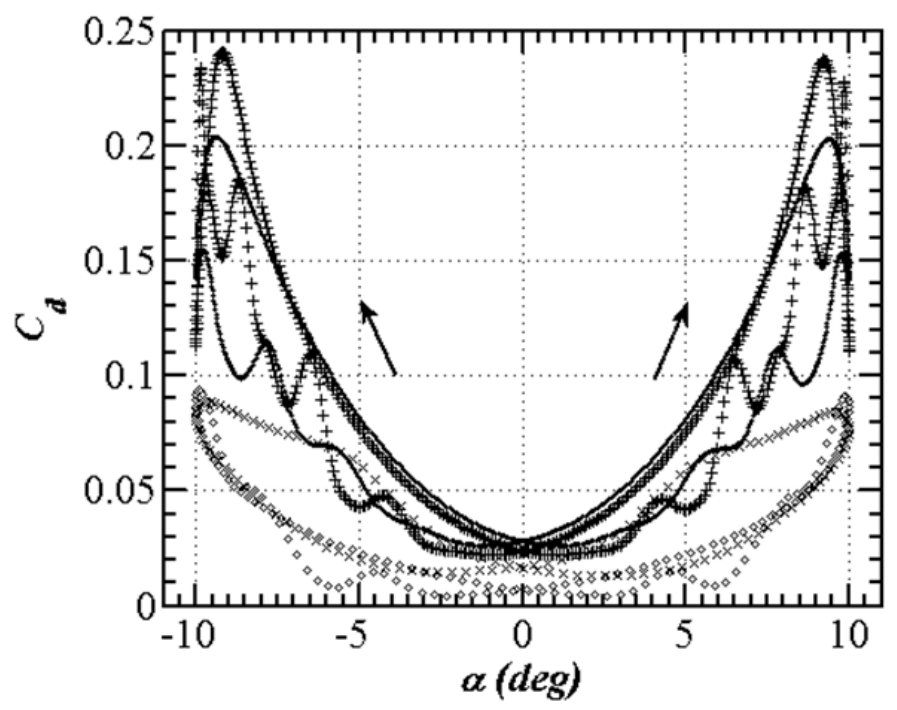

Figure 4.20: Drag coefficient $\left(C_{d}\right)$ versus angle of attack $(\alpha), d=10^{\circ}, k=0.1:(-), \operatorname{Re}=$ $555 ;(+), \operatorname{Re}=1000 ;(\diamond), \operatorname{Re}=2000 ;(\times), \operatorname{Re}=5000$.

lift and drag coefficients. The parameters increase or decrease the aerodynamic force peak values depending on the surrounding flow structure. Hysteresis loops are also broadened or narrowed due to similar reasons. It is also observed that $d, k$ and Re are effective in changing the number, strength, and even the development angle of the generated vortical patterns. 
Moreover, a pattern called figure-of-eight phenomenon is observed in the predicted force coefficients at $\operatorname{Re}=2000$, which is eliminated at $\operatorname{Re}=5000$. Similar effects are noticed in the drag coefficient results, but the minimum drag coefficient is not affected substantially by the investigated parameters except at the high amplitudes of oscillation and high Reynolds numbers. Numerical modeling of the flow field characteristics of a LRN plunging airfoil is investigated and will be presented in the following chapter. 


\section{Chapter 5}

\section{Numerical modeling of the flow field characteristics of a LRN plunging airfoil}

In what follows, the flow field and vortical patterns around a thin ellipsoidal plunging airfoil are examined in detail with and without freestream velocity, and the effects of Reynolds (Re) and Strouhal (St) numbers on the flow characteristics are explored. It is shown that both Re and St increase the aerodynamic performance in non-zero freestream velocity simulations. Increasing Re and St causes the airfoil to generate thrust for some time intervals of the plunging period. This thrust generation is penalized with higher peaks of drag coefficient when St increases. However, the same penalty in the Re effect simulations is negligible compared to that of the St effects. Increasing St causes the airfoil to experience negative pitching moment with higher peak values for longer time intervals, however, Re does not change the time at which negative pitching moment is exerted on the airfoil, but the peaks of pitching moment depend on the governing Re. The lift coefficient changes noticeably versus St, where there is significant lead/lag at the peak lift coefficient for zero-freestream velocity simulations. Reynolds number effects on the lift coefficients mostly occur around the time 
at which the peak lift coefficient is obtained for both zero and non-zero freestream velocity cases. All of these effects are caused by the complex vortical patterns around the airfoil, described throughout the present chapter.

\subsection{Introduction}

Plunging motion is another prevalent unsteady condition observed in applications such as MAVs. Unfortunately most of conducted LRN studies are focused on the harmonic flapping motions and less attention is paid to the LRN plunging and pitching motions. As the occurring fluid phenomena are affected by the kinematic pattern, the plunging studies are needed to reveal the dependence. The phenomena, which are mostly viscous, such as LEVs and dynamic stall, are the major mechanisms of force and moment generation in LRN unsteady flows. The importance of LEVs and TEVs in LRN plunging motions are shown in the studies of Lewin and Haj-Hariri [81] and Prangemeier et al. [50]. The downstream wake of a plunging airfoil also plays a significant role in the flow behavior and force coefficients. Triantafyllou et al. [53], Freymuth [51], Lai and Platzer [52], Heathcote and Gursul [9] and Young and Lai [71] investigated plunging airfoils' downstream wake and showed its dependence on the plunging motion unsteady parameters. Andro and Jacquin [76] studied the flow field of a heaving airfoil numerically, and analyzed the significance of LEVs, added mass and the wake-capturing mechanisms. They also showed how these phenomena change when subjected to different frequencies of oscillations. The objective of the present chapter is to improve the existing understanding of LRN plunging airfoil flow characteristics and its dependency on the governing flow and system parameters. To this end, the flow field of an elliptic airfoil under harmonically heaving motion is simulated using CFD based on FVM and incompressible N-S equations. In the present 2-D study, the effects of Reynolds and Strouhal numbers on the instantaneous lift, drag and moment coefficients at both zero and nonzero freestream velocities are investigated (Section 5.5). These parameters have been shown to 
be of great importance to the fluid dynamics characteristics of pitching [10], plunging [135], and flapping [6] airfoils.

\subsection{Solution method}

Equations 3.9 govern the flow field of a LRN plunging airfoil. In this chapter, the effects of St and Re (Equations 3.10) on the force and moment coefficients of an ellipsoidal airfoil under heaving oscillations are investigated. The computational domain is discretized using an O-type mesh. The far-field boundary with symmetry condition is set to $30 c$ in order to minimize its undesired effects on the airfoil's surrounding flow field. The simulations in the present chapter are conducted for both zero and nonzero freestream velocities. The inlet and outlet are set to symmetry condition in zero freestream velocity simulations. For nonzero freestream simulations, the inlet is set to a velocity Dirichlet and zero-gradient pressure condition, and the outlet is set to freestream pressure and zero-gradient velocity. The airfoil surface is set to a no-slip boundary condition. The computations utilize $48 \times 10^{3}$ cells and 2000 time steps per period. This choice was made after extensive grid and time step independence analyses. Fig. 5.1 shows the schematic of the airfoil and the computational domain.

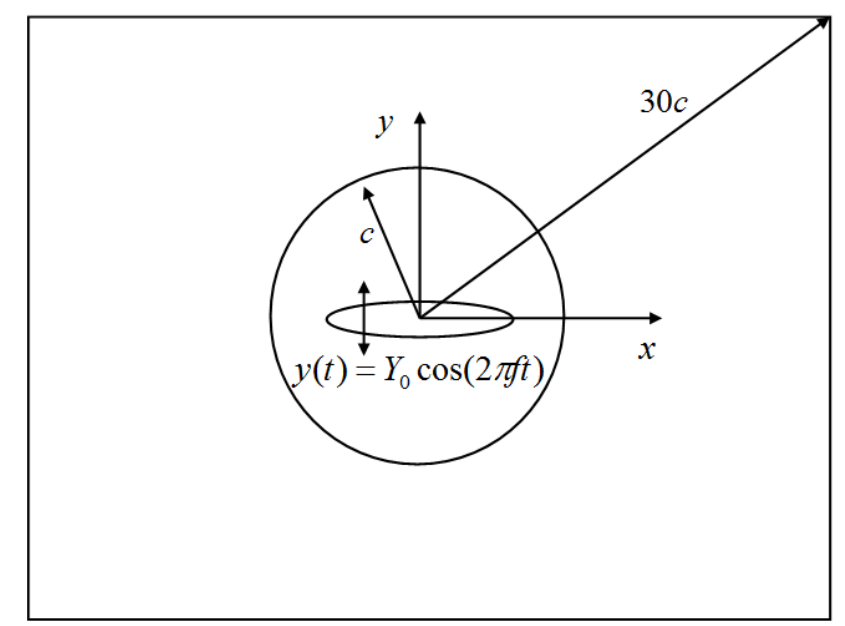

Figure 5.1: Schematic of the airfoil and the computational domain. 
FVM is used to investigate the aerodynamic characteristics of an ellipse with $2 \%$ thickness. Thin airfoils are the commonly used lifting surfaces in Micro Aerial Vehicle (MAV) applications and LRN flows. The computations utilize a second order central differencing scheme (CDS) for the convective and diffusive terms and a second order Euler implicit scheme for temporal discretizations. The resulting linear system of equations is treated with a Preconditioned Conjugate Gradient (PCG) solver, and the Semi-Implicit Method for Pressure Linked Equations (SIMPLE) algorithm is used for the pressure-velocity coupling. The mesh motion is based on the Laplace smoothing mesh motion algorithm [107]. The airfoil plunging equation of motion is given by:

$$
y(t)=Y_{0} \cos (2 \pi f t),
$$

where $y(t)$ is the vertical position vector, $Y_{0}$ is the amplitude of plunging oscillations, and $f$ is the frequency of oscillations.

\subsection{Reference velocity, force and moment coefficients calculations}

The flow field of an airfoil under heaving oscillations $\left(y(t)=Y_{0} \cos (2 \pi f t)\right)$ is simulated with and without freestream velocity $\left(U_{0}\right.$ in Fig. 5.2). The reference velocity $\left(U_{\text {ref }}\right)$ in the calculation of Re and St (Equations 3.10) is either the maximum plunging velocity ( $U_{r e f}=$

$\left.\dot{y}_{\max }\right)$ when there is no freestream velocity or the freestream velocity $\left(U_{r e f}=U_{0}\right)[70]$ when there is oncoming flow velocity.

The unsteady force and moment coefficients also need a reference velocity. The instantaneous lift $\left(C_{l}\right)$, drag $\left(C_{d}\right)$, and moment $\left(C_{m}\right)$ coefficients are obtained using the following equations:

$$
C_{l}(t)=\frac{F_{y}(t)}{\frac{1}{2} \rho c U^{2}} ; \quad C_{d}(t)=\frac{F_{x}(t)}{\frac{1}{2} \rho c U^{2}} ; \quad C_{m}(t)=\frac{M(t)}{\frac{1}{2} \rho c^{2} U^{2}},
$$

where $F_{y}(t)$ and $F_{x}(t)$ are the instantaneous forces in $y$ (lift) and $x$ (drag) directions, re- 


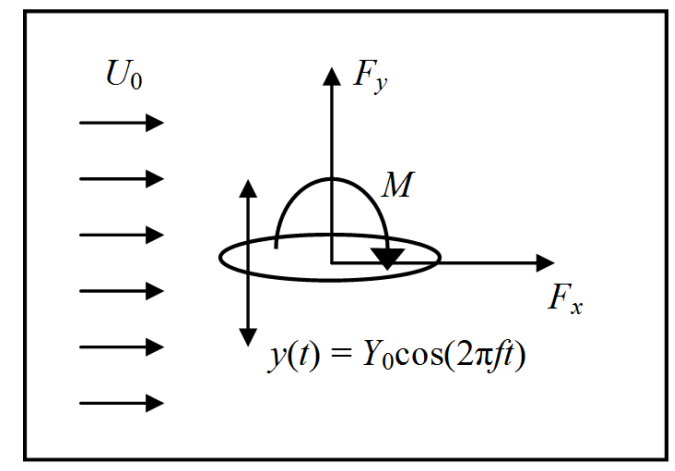

Figure 5.2: Schematic of the plunging airfoil and force and moment signatures.

spectively, $M(t)$ is the instantaneous pitching moment calculated at $x / c=0.5, c$ is the chord-length and $\rho$ is the density. $U$ is calculated using the following equation:

$$
U=\sqrt{U_{0}^{2}+\dot{y}_{\max }^{2}}
$$

\subsection{Evaluation of the results}

In what follows (Subsection 5.4.1), several simulations are first performed to investigate the grid and time step resolutions and to validate the results using the available data in the literature. In the following Subsection (5.4.2), three case studies (also reported by Williamson [136], Guilmineau and Queutey [137] and Lian and Shyy [138] and Young [139]) are then carried out to further validate the proposed numerical approach and settings. The main numerical results and discussions are presented in the next Section (Section 5.5).

\subsubsection{Mesh and time step sensitivity analyses}

In order to obtain grid and time independent solutions, several cases with different grid size and time step resolutions are examined. The results of these analyses are shown in Figs. 5.3(a) and 5.3(b) for the plunging ellipsoidal airfoil at $\mathrm{St}=0.16$ and $\mathrm{Re}=100$, when $U_{0}=0$. First, the grid sensitivity is investigated using 24, 48, and $96\left(\times 10^{3}\right)$ cells and 2000 time steps per plunging cycle. Figure 5.3(a) shows the obtained $C_{l}$ versus $\tau$ for a half stroke 
of the airfoil motion, where the second half stroke has been omitted because it has the same profile as that of the first half but with the opposite values. As shown, the difference between 48 and $96\left(\times 10^{3}\right)$ cell cases is negligible. Hence, $48 \times 10^{3}$ is sufficiently refined to yield grid independent solutions. The time step is also varied for 1000, 2000 and 4000 time steps within a cycle having $48 \times 10^{3}$ cells. The results show that the difference between the lift coefficients of 2000 and 4000 time step cases is negligible, Fig. 5.3(b). Therefore, the computations are performed using $48 \times 10^{3}$ cells and 2000 time steps.

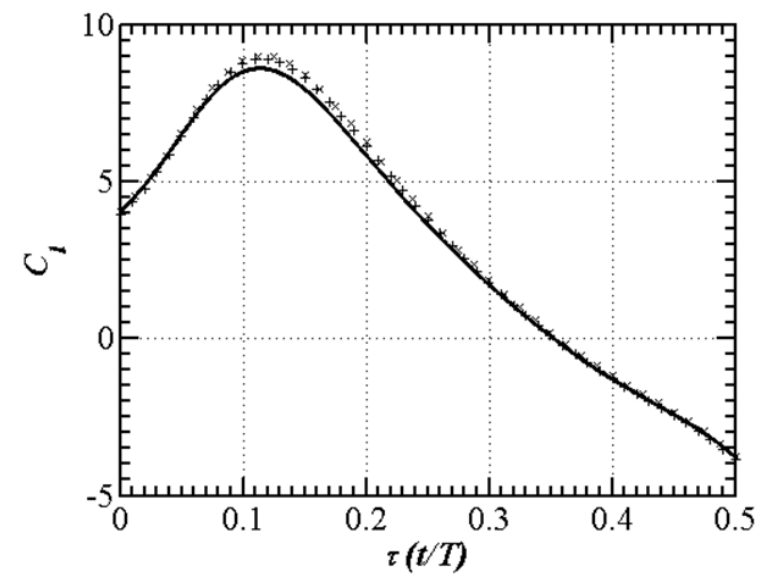

(a)

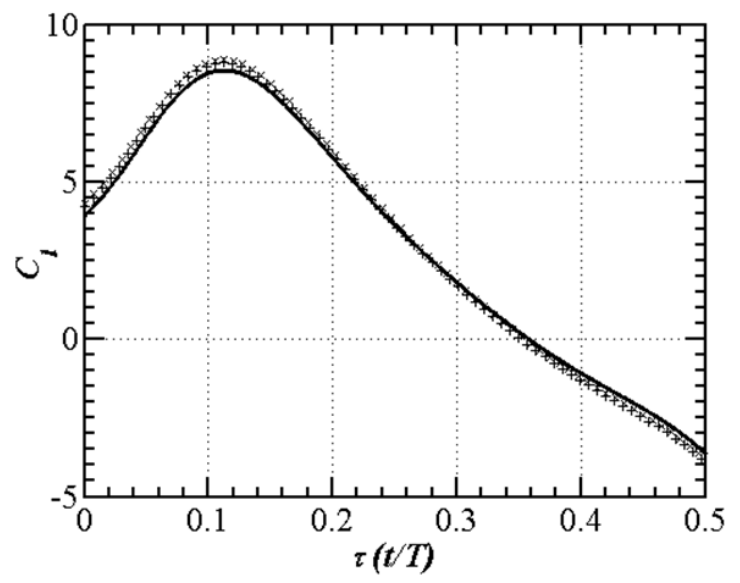

(b)

Figure 5.3: Instantaneous lift coefficient $\left(C_{l}\right)$ versus nondimensional time $(\tau)$ for $\mathrm{St}=0.16$, Re $=100$, and $U_{0}=0,(\mathrm{a}):(-) 24 \times 10^{3},(+) 48 \times 10^{3},(\times) 96 \times 10^{3}$ cells with 2000 time steps;(b): (-) 1000, (+) 2000, (×) 4000 time steps with $48 \times 10^{3}$ cells.

\subsubsection{Validation studies}

The first validation case, also studied by Williamson [136], is the flow field of a static cylinder at $\operatorname{Re}=150$. The mean drag coefficient $\left(\bar{C}_{d}\right)$ is the benchmark for the comparison. The present simulation gives $\bar{C}_{d}=1.375$ which is in very close agreement (3\% difference) with $\bar{C}_{d}=1.334$, reported by Williamson [136]. Figure 5.4 shows the numerically computed instantaneous $C_{d}$ versus time $(t)$ in the present simulations.

The second validation case is a plunging cylinder at $\mathrm{Re}=185$, reported by Guilmineau 


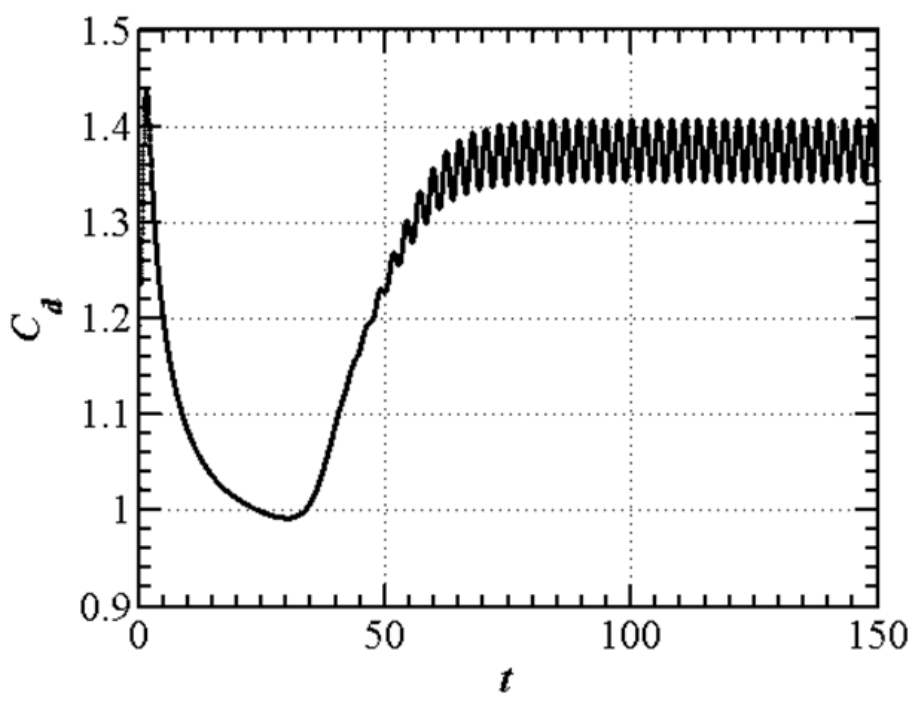

Figure 5.4: Instantaneous drag coefficient $\left(C_{d}\right)$ versus time $(t)$ for a static cylinder at $\mathrm{Re}=$ 150.

and Queutey [137]. The motion is governed by the following equation:

$$
y(t)=-A_{e} \sin \left(2 \pi f_{e} t\right)
$$

with $A_{e}=0.2 c$ ( $c$ being the cylinder diameter) and $f_{e}=0.154$. The resulting $\bar{C}_{d}$ is 1.25 which is in close agreement (4\% difference) with that reported by Guilmineau and Queutey [137], $\bar{C}_{d}=1.2$. Figure 5.5 shows the obtained instantaneous $C_{d}$ and $C_{l}$ versus $t$.

Finally the plunging oscillations of a NACA0012 airfoil is simulated, also studied by Lian and Shyy [138] and Young [139]. The nondimensional amplitude of oscillations $\left(Y_{0} / c\right)$ is 0.0125 and Strouhal number, defined as $\mathrm{St}=\frac{2 f c Y_{0}}{U_{\infty}}$ by Lian and Shyy [138], is St $=$ 0.03. Figs. 5.6 shows the comparison between the calculated $C_{l}$ and $C_{d}$, respectively, of the present simulations and those of the considered references. As can be seen, the lift and drag coefficients are in very good agreement with those of Lian and Shyy [138] and Young [139]. 


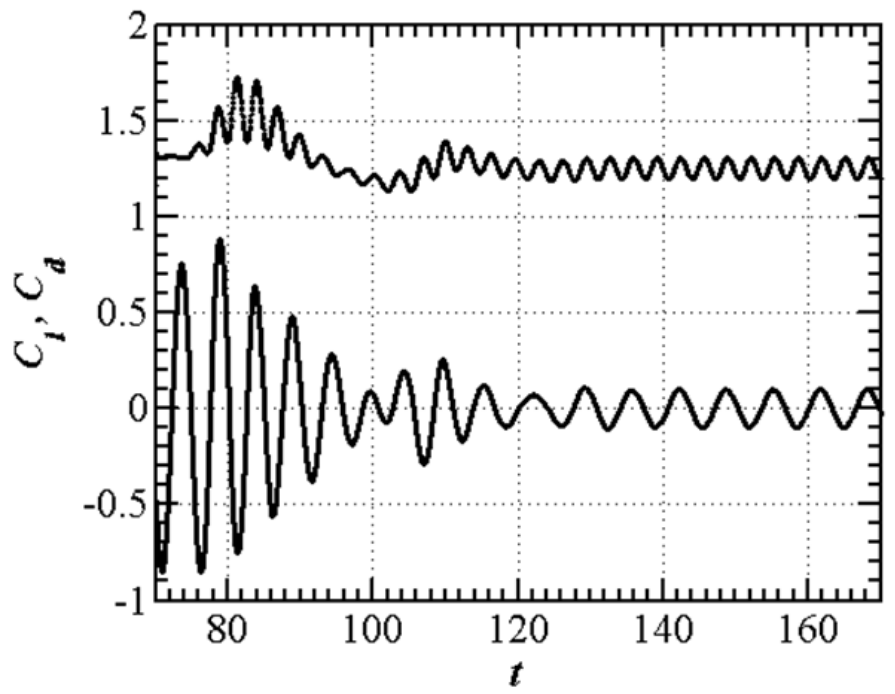

Figure 5.5: Instantaneous lift and drag coefficients $\left(C_{l}\right.$ and $\left.C_{d}\right)$ versus time $(t)$ of a plunging cylinder following Equation 5.4 at $\operatorname{Re}=185, A_{e}=0.2 c$ ( $c$ being the cylinder diameter $)$ and $f_{e}=0.154$.
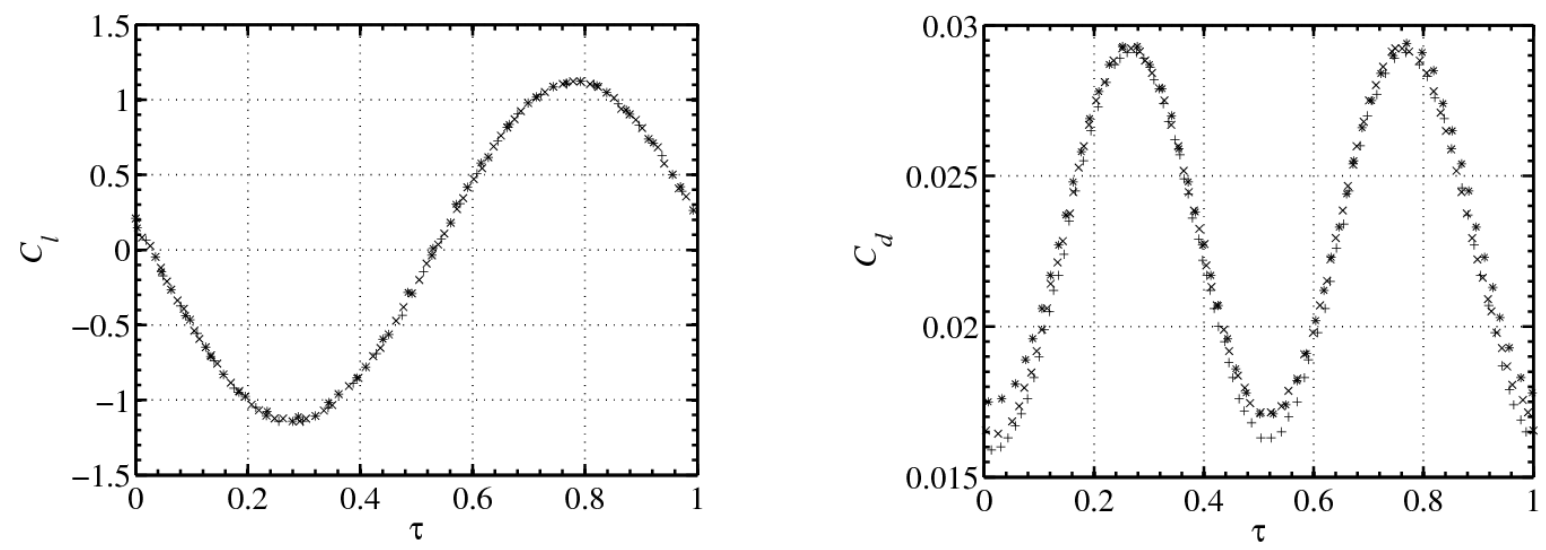

Figure 5.6: Instantaneous lift coefficient (left) and drag coefficient (right) versus $\tau$ for a plunging NACA0012 airfoil with $Y_{0} / c=0.0125$ and St $=0.03:\left({ }^{*}\right)$, Lian and Shyy [138]; $(+)$, Young [139]; $(\times)$, present simulations.

\subsection{Evaluation of the results}

In this section, numerical simulations of a thin plunging ellipse $(t / c=0.02)$ are conducted. The effects of St and Re (based on airfoil's chord-length, St $=\frac{f c}{U_{r e f}}$ ) on the force and moment signatures $\left(C_{l}, C_{d}\right.$ and $\left.C_{m}\right)$ and on the vortical patterns surrounding the airfoil, for both 
Table 5.1: Summary of experimental and numerical plunging studies in the literature.

\begin{tabular}{|c|c|}
\hline Author & Performed investigation \\
\hline Andro and Jacquin [76] & $\begin{array}{l}\text { 2-D, numerical }(\mathrm{N}-\mathrm{S}), \mathrm{NACA} 0012, \mathrm{Re}=1000 \text {, effect of } \\
\text { St }\end{array}$ \\
\hline Chandar and Damodaran [79] & $\begin{array}{l}\text { 2-D, numerical (N-S), NACA0012, Re }=10^{4}, 4.5 \times 10^{4} \text {, } \\
\text { effect of airfoil TE }\end{array}$ \\
\hline Hamdani and Sun [70] & $\begin{array}{l}2-\mathrm{D} \text {, numerical, } \mathrm{Re}=100 \text {, effect of acceleration/decel- } \\
\text { eration }\end{array}$ \\
\hline Heathcote and Gursul [9] & $\begin{array}{l}\text { 2-D, 3-D, experimental, } \mathrm{Re}=10^{4}, 2 \times 10^{4}, 3 \times 10^{4}, k= \\
0-14\end{array}$ \\
\hline Lai and Platzer [52] & $\begin{array}{l}2-\mathrm{D}, \text { experimental (LDV), NACA0012, } \mathrm{Re}=500- \\
2.1 \times 10^{4}\end{array}$ \\
\hline Lentink and Gerritsma [80] & $\begin{array}{l}\text { 2-D, numerical }(\mathrm{N}-\mathrm{S}), \mathrm{Re}=150,192 \text {, effect of different } \\
\text { airfoil shapes }\end{array}$ \\
\hline Lewin and Haj-Hariri [81] & $\begin{array}{l}2-\mathrm{D} \text {, numerical }(\mathrm{N}-\mathrm{S}) \text {, elliptic airfoil, } 0.25<\mathrm{St}<0.48 \\
\operatorname{Re}=500\end{array}$ \\
\hline Lua et al. [48] & $\begin{array}{l}2-\mathrm{D}, \text { experimental (DPIV), elliptic airfoil, } \mathrm{Re}=1000, k \\
=1-2\end{array}$ \\
\hline Pederzani and Haj-Hariri [82] & $\begin{array}{l}\text { 2-D, numerical (N-S), NACA0012, Re }=500, k h=0.8 \text {, } \\
1\end{array}$ \\
\hline Prangemeier et al. [50] & 2-D, experimental (PIV), Re $=3 \times 10^{4}, k=0.25$ \\
\hline Triantafyllou et al. [53] & $2-\mathrm{D}$, experimental, $\mathrm{Re}=3000, \mathrm{St}=0.25-0.35$ \\
\hline Young and Lai [71] & 2-D, numerical (N-S), Re $=2 \times 10^{4}$, effect of $k$, St \\
\hline
\end{tabular}

zero and nonzero freestream velocities, are investigated. The parametric study corresponds to LRN regime, which is not studied well in the literature. Table 5.1 shows a summary of some of the existing studies in the literature.

\subsubsection{Effect of the Strouhal number without freestream flow}

Here, the effect of St on the airfoil lift coefficients at Re $=100$ is investigated for St $=0.32$, 0.16 and 0.11 , corresponding to $Y_{0}=0.5 c, c$ and $1.5 c$, respectively. Figure 5.7 shows $C_{l}$ versus $\tau$ for one plunging cycle. As can be seen, increasing $Y_{0}$ (decreasing St) decreases the maximum lift coefficient $\left(C_{l_{\max }}\right)$. That is, $C_{l_{\max }}=13.2,8.8$ and 6.6 , at $\mathrm{St}=0.32,0.16$ and 0.11 , respectively. This decreasing effect is associated with a lead in the generation of $C_{l_{\max }}$. The maximum lift coefficient is obtained at $\tau=0.2,0.12$ and 0.1 at $\mathrm{St}=0.32,0.16$ and 0.11 , respectively. Similarly, the magnitude of the minimum lift coefficient $\left(C_{l_{\min }}\right)$ is decreased, 
and $C_{l_{\min }}$ is obtained at $\tau=0.7,0.62$, and 0.6 for $\mathrm{St}=0.32,0.16$, and 0.11 . Examining the magnitudes of $C_{l_{\max }}$ in Fig. 5.7 also confirms that the lift coefficients are dependent on St rather than the amplitude of oscillations. That is, examining the calculated $C_{l_{\max }}$ values shows that their percentage of decrease is proportional to the St values rather than $Y_{0}$. Figures 5.8(a-c) show the pressure field around the airfoil when $C_{l_{\max }}$ is obtained. In these figures, the black color shows low pressure, while the white-colored areas indicate high pressure. As can be seen, the pressure field at $\mathrm{St}=0.32$ has two symmetric low-pressure zones at the LE and TE of the airfoil. These regions show LE and TE vortices, which enhance the lift coefficient. The vortices are also stretched and deflected towards the lower surface of the airfoil and surround the high-pressure zone. Decreasing Strouhal number from St $=0.32$ to 0.16 generates smaller low-pressure zones with lower strength (brighter color), which results in the decreased $C_{l_{\max }}$. The LE and TE vortices are further deflected into the downstream of the airfoil when St is changed from 0.16 to 0.11 , which is the reason for the decreased $C_{l_{\max }}$. It should be mentioned that despite having a symmetrical plunging motion, Fig.5.7 shows that the lift values do not have a symmetrical pattern. The reason for this behavior should be studied in more detail as a future work.

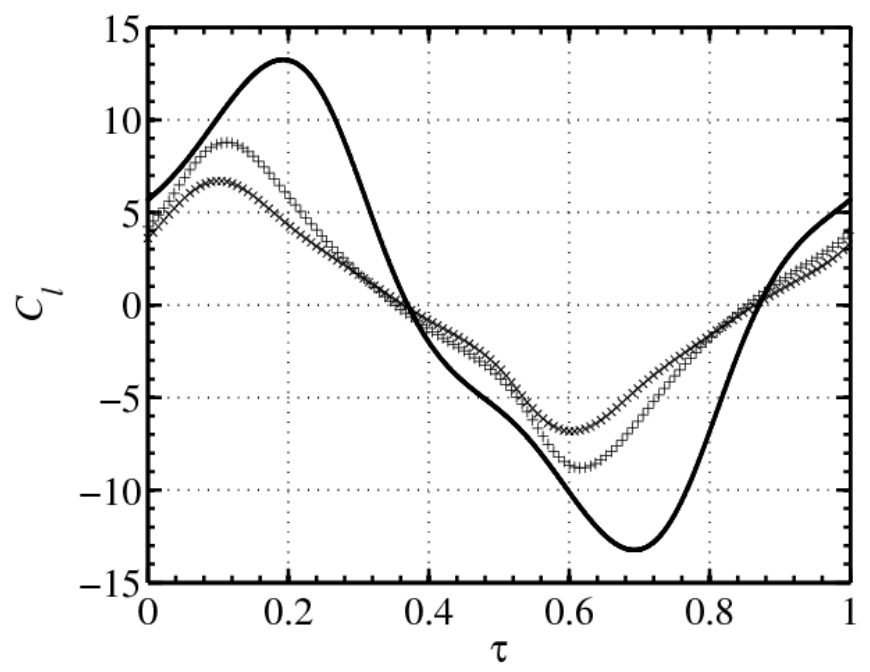

Figure 5.7: Instantaneous lift coefficient $\left(C_{l}\right)$ versus nondimensional time $(\tau)$ when $\operatorname{Re}=$ 100 and $U_{0}=0:(-) \mathrm{St}=0.32,(+) \mathrm{St}=0.16,(\times) \mathrm{St}=0.11$. 


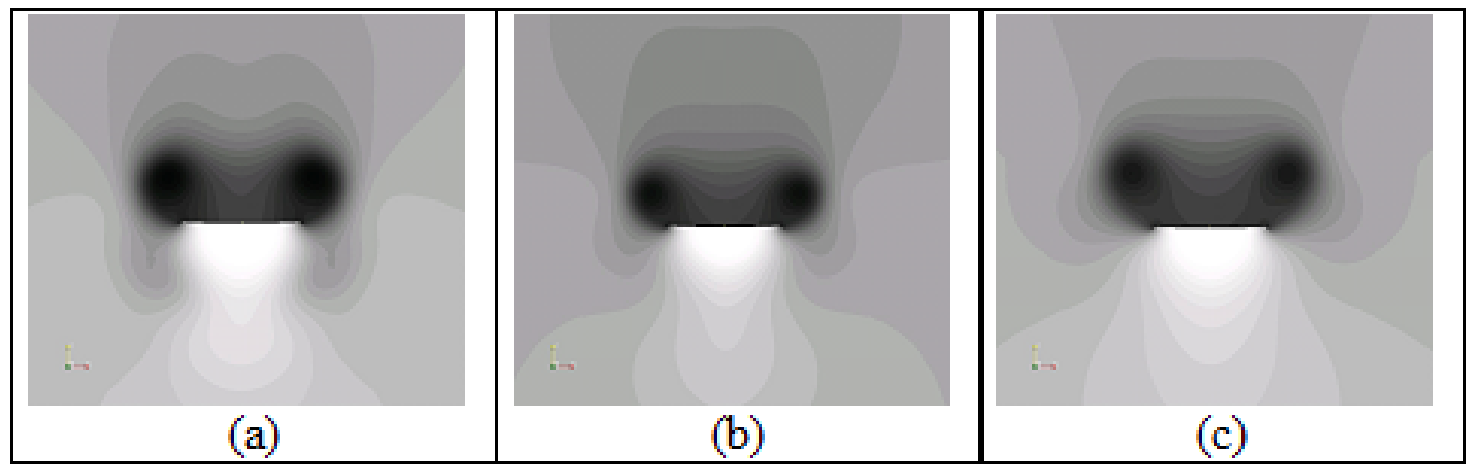

Figure 5.8: Pressure contours when maximum lift coefficient $\left(C_{l_{\max }}\right)$ is achieved at $\operatorname{Re}=100$ and $U_{0}=0$ : (a) St $=0.32$, (b) St $=0.16$, (c) St $=0.11$.

\subsubsection{Effect of the Strouhal number with freestream flow}

In this subsection, the effect of St on $C_{l}, C_{d}$ and $C_{m}$ of the plunging airfoil is investigated for St $=0.08,0.16$ and 0.32 , where $U_{\text {ref }}$ is the freestream velocity. According to the following discussions, it is found that increasing St increases peak lift coefficients significantly. The magnitudes of the peak lift coefficients are almost equal in up- and down-strokes. However, at the highest investigated St, secondary peaks are induced into the instantaneous lift followed by smaller peaks in down-stroke compared to that in the up-stroke. Moreover, increasing St decreases drag coefficients such that at the highest considered St, thrust is generated by the airfoil, penalized by the increased maximum drag coefficients. The peaks of the pitching moment coefficient also strongly depend on the governing St, and the time interval at which the airfoil is subjected to negative or positive moment is changed with respect to

St. Moreover, calculation of the aerodynamic performance $\left(\frac{\bar{C}_{l}}{\bar{C}_{d}}\right)$ for up- and down-strokes shows that St increases $\frac{\bar{C}_{l}}{\bar{C}_{d}}$.

Fig. 5.9 shows $C_{l}$ versus $\tau$. It can be observed that increasing St increases $C_{l_{\max }}$ in both up- and down-strokes. The instantaneous $C_{l}$ at $\mathrm{St}=0.32$ shows that there is a secondary peak $C_{l}$ at $\tau \approx 0.6$. Moreover, the close examination of the peaks of $C_{l}$ at $\mathrm{St}=0.32$ shows that $\left|C_{l_{\text {min }}}\right|$ is less than $\left|C_{l_{\max }}\right|$, which is accompanied by the generation of the observed secondary peak. Moreover, the influence of St on $C_{l_{\max }}$ is more than that on the $C_{l_{\min }}$, which could also be related to the generation of the inflection point at $\tau \approx 0.6$. 


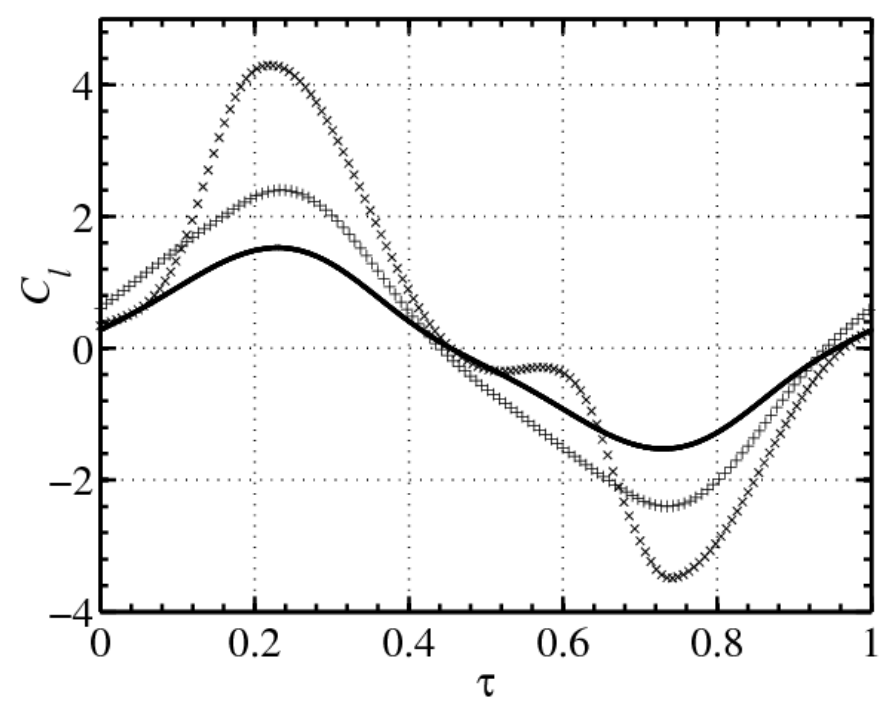

Figure 5.9: Instantaneous lift coefficient $\left(C_{l}\right)$ versus nondimensional time $(\tau)$ for $\operatorname{Re}=100$, $Y_{0}=c$ and $U_{0} \neq 0:(-)$ St $=0.08,(+)$ St $=0.16,(\times)$ St $=0.32$.

Figure 5.10 shows $C_{d}$ versus $\tau$. Increasing St from 0.08 to 0.16 is beneficial to $C_{d}$ as the drag coefficient is reduced. The changes resemble a down-shift of $C_{d}$ with $C_{d_{\max }}=0.26$ and 0.13 and $C_{l_{\min }}=0.127$ and 0.01 for St $=0.08$ and 0.16 , respectively. Further increase of St from 0.16 to 0.32 shows an interesting behavior. The drag producing airfoil at St $=0.08$ and 0.16 is capable of producing thrust at $\mathrm{St}=0.32$ between $0.2 \leq \tau \leq 0.57$ and $0.76 \leq \tau \leq 1$. The generated thrust could be of high importance to applications such as forward flight and fish-like locomotion; however, this benefit is gained with the expense of increased $C_{d}$ around the second $C_{d_{\max }}$, between $\mathrm{St}=0.16$ and 0.32 . It should also be mentioned that $C_{d_{\max }}$ and $C_{d_{\text {min }}}$ are obtained with negligible lead and lag, respectively, between $\mathrm{St}=0.08$ and 0.16. However, all the peak drag coefficients $\left(C_{d_{\max }}\right.$ and $\left.C_{d_{\min }}\right)$ are obtained with significant lag at $\mathrm{St}=0.32$. The effect of St on $C_{d_{\min }}$ (drag coefficient at $\mathrm{St}=0.08$ and 0.16 and thrust coefficient at $\mathrm{St}=0.32$ ) is investigated in more details on the airfoil's vortical patterns in Figs. 5.11(a-c). It is seen from these figures that by increasing St, the LEV is strengthened and leans towards the airfoil surface. The TEV is also strengthened, where these changes cause the first $C_{d_{\min }}$ to decrease versus St and eventually converts into a thrust coefficient $\left(C_{d_{\min }} \leq 0\right)$ at $\mathrm{St}=0.32$ 


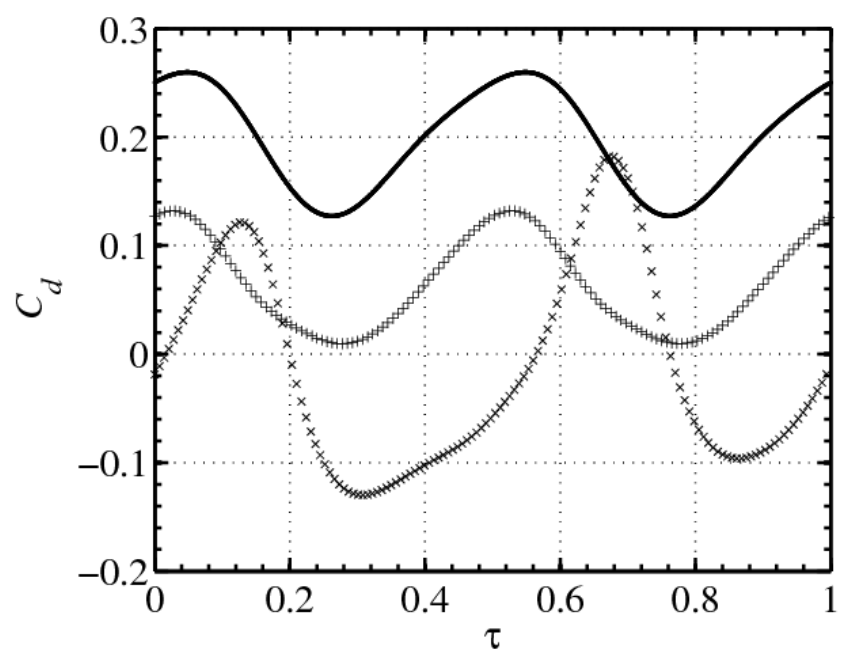

Figure 5.10: Instantaneous drag coefficient $\left(C_{d}\right)$ versus nondimensional time $(\tau)$ for $\operatorname{Re}=$ $100, Y_{0}=c$ and $U_{0} \neq 0:(-) \mathrm{St}=0.08,(+) \mathrm{St}=0.16,(\times) \mathrm{St}=0.32$.

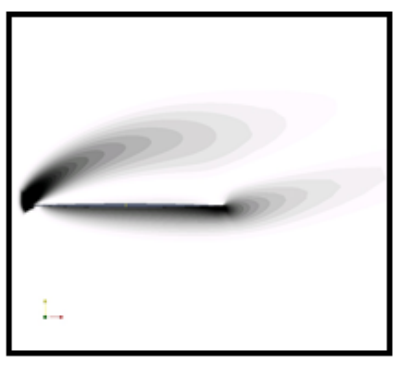

(a)

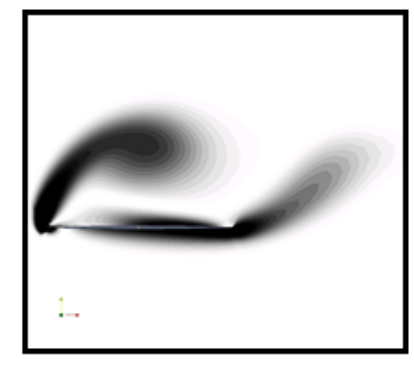

(b)

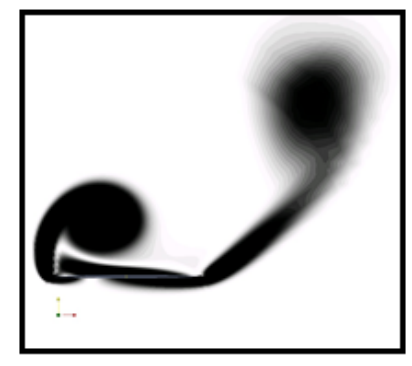

(c)

Figure 5.11: Vortical patterns around the airfoil when first maximum drag coefficient $\left(C_{d_{\min }}\right)$ is obtained for $Y_{0}=c, \mathrm{Re}=100$ and $U_{0} \neq 0$ : (a), St $=0.08$; (b), St $=0.16$; (c), St $=0.32$.

Figure 5.12 shows $C_{m}$ versus $\tau$. As it is observed, St has significant effects on both $C_{m_{\text {min }}}$ and $C_{m_{\max }}$, where $\left|C_{m_{\min }}\right|$ is consistently increased, which could be a negative trend from a stability perspective. However, the effect of St on $\left|C_{m_{\max }}\right|$ does not follow a constant trend, meaning that $\left|C_{m_{\max }}\right|$ initially decreases between $\mathrm{St}=0.08$ and 0.16 , and then increases between $\mathrm{St}=0.16$ and 0.32 . Moreover, the time intervals in which the airfoil is subjected to negative and positive pitching moment coefficients, changes with respect to the governing St. The airfoil encounters negative instantaneous $C_{m}$ between $0<\tau<0.32,0<\tau<0.38$ and $0<\tau<0.54$ for St $=0.08,0.16$ and 0.32 , respectively, and it undergoes positive $C_{m}$ 
for almost the rest of plunging cycle at the three investigated St values, with the exception of a small period of negative $C_{m}$ at the end of $\mathrm{St}=0.32$. Hence, by increasing $\mathrm{St}$, the surrounding fluid flow applies nose-down pitching moment on the airfoil for a longer time interval.

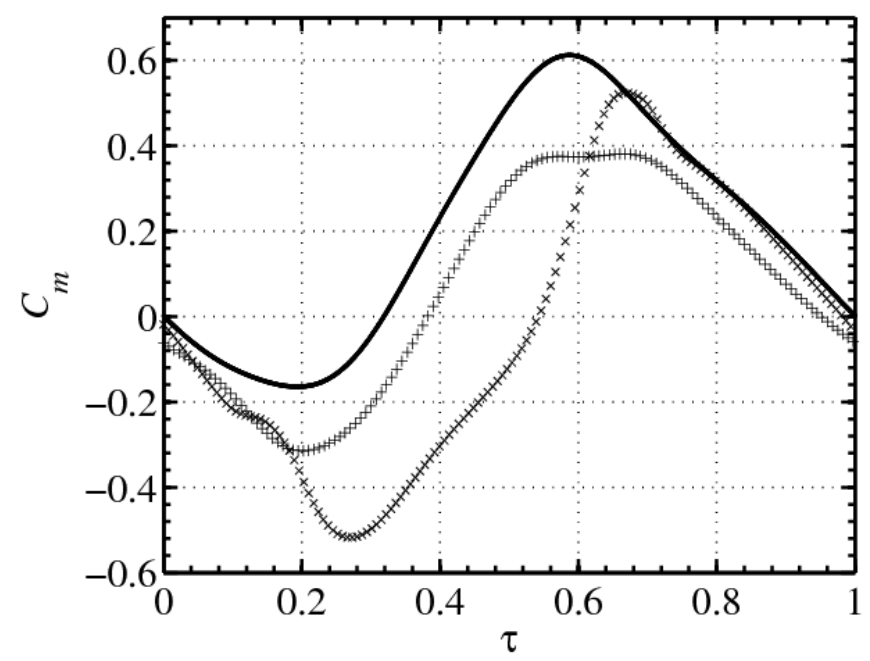

Figure 5.12: Instantaneous pitching moment coefficient $\left(C_{m}\right)$ versus nondimensional time $(\tau)$ for $\mathrm{Re}=100, Y_{0}=c$ and $U_{0} \neq 0:(-) \mathrm{St}=0.08,(+) \mathrm{St}=0.16,(\times) \mathrm{St}=0.32$.

The average lift and drag coefficients $\left(\bar{C}_{l}\right.$ and $\left.\bar{C}_{d}\right)$ are studied and listed in Table 5.2. The mean force coefficients are calculated for the up- and down-strokes separately. As it can be seen, increasing St increases $\bar{C}_{l}$, but decreases $\bar{C}_{d}$, resulting in a higher aerodynamic performance $\left(\frac{\bar{C}_{l}}{\bar{C}_{d}}\right)$ in each half stroke.

Table 5.2: Mean lift and drag coefficients $\left(\bar{C}_{l}\right.$ and $\left.\bar{C}_{d}\right)$ for the investigated St where Re $=$ $100, Y_{0}=c$ and $U_{0} \neq 0$

\begin{tabular}{ccc}
\hline \hline St & Upstroke & Downstroke \\
\hline 0.08 & $\bar{C}_{l}=0.838$ and $C_{d}=0.197$ & $\bar{C}_{l}=0.837$ and $\bar{C}_{d}=0.197$ \\
0.16 & $\bar{C}_{l}=1.304$ and $\bar{C}_{d}=0.065$ & $\bar{C}_{l}=1.303$ and $\bar{C}_{d}=0.065$ \\
0.32 & $\bar{C}_{l}=1.977$ and $\bar{C}_{d}=0.032$ & $\bar{C}_{l}=1.447$ and $\bar{C}_{d}=0.005$
\end{tabular}




\subsubsection{Effect of the Reynolds number}

In what follows, the effect of Reynolds number $(\mathrm{Re})$ on $C_{l}, C_{d}$ and $C_{m}$ of the plunging airfoil is investigated for $\operatorname{Re}=100,200$ and 500, with freestream velocity. The Re effect on the $C_{l}$ for $\operatorname{Re}=50$ and 100 without freestream velocity is also studied. The vortical patterns around the airfoil are also investigated at the points of interest. It is found, according to the following discussions, that based on the governing $\operatorname{Re}\left(U_{0} \neq 0\right)$, the airfoil could generate drag and thrust during a plunging cycle, and increasing Re can cause the airfoil to produce thrust for a longer period of the oscillations with the expense of a slightly higher peak of the drag coefficient, i.e., lower thrust. Moreover, increasing Re could increase or decrease the pitching moment coefficient, depending on the position of the airfoil during its plunging motion. However, the time interval at which the airfoil is subjected to negative or positive moment is not a function of Re. Although Re effects on the lift coefficient are minimal compared to those on drag and moment coefficients, these effects occur at different time intervals of the plunging motion. The corresponding vortical patterns show very complex LEV and TEV structures and their interactions. Moreover, it is found that increasing

Re increases the aerodynamic performance $\left(\frac{\bar{C}_{l}}{C_{d}}\right)$, where $\bar{C}_{l}$ and $\bar{C}_{d}$ are the average lift and drag coefficients within a plunging cycle and are calculated for the up- and down-strokes separately. In addition, the simulations without freestream velocity also show that Re only affects $C_{l}$ in the regions around the peak lift coefficient.

Figure 5.13 shows $C_{l}$ versus $\tau$ for $\operatorname{Re}=50$ and 100, where Re has negligible effects on $C_{l}$ in most part of the cycle except for a narrow range of $C_{l}$ near the $C_{l_{\max }}$.

Figure 5.14 shows $C_{l}$ versus $\tau$ when $U_{0} \neq 0$. Increasing Re from 100 to 200 increases the magnitude of peak lift coefficients. For example, $C_{l_{\max }} \approx 2.4$ and 2.7 at $\mathrm{Re}=100$ and 200 , respectively. In addition to the increased $C_{l_{\max }}$, the instantaneous $C_{l}$ is higher at Re $=200$ for. However, it is observed that Re does not increase $C_{l_{\max }}$ from $\mathrm{Re}=200$ to 500 , and the difference between the real time $C_{l}$ at $\operatorname{Re}=200$ and 500 mostly occurs when $0<$ $\tau<0.17$ and $0.5<\tau<0.67$. This behavior could be interpreted as if the lowered viscous 


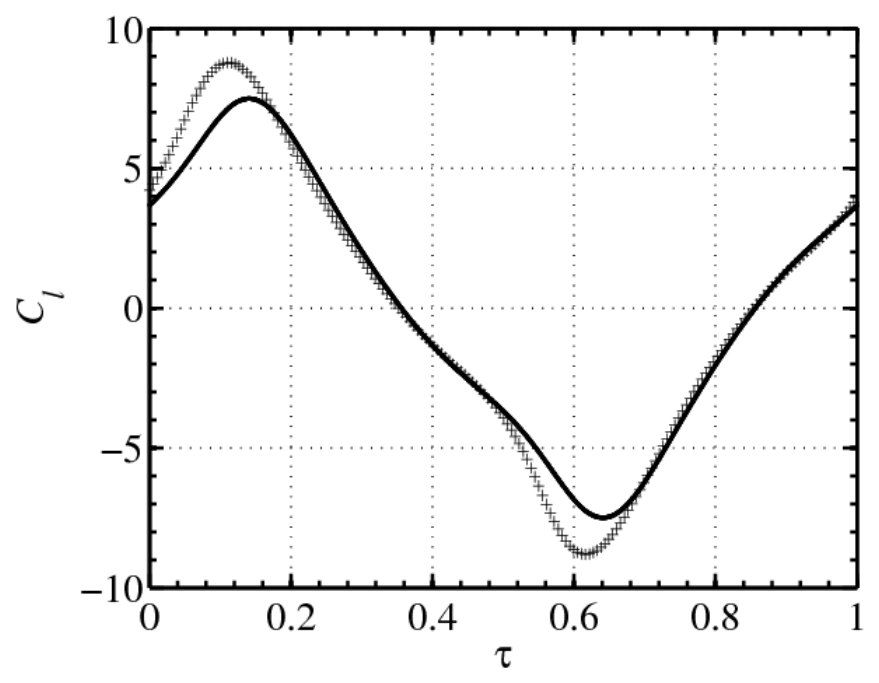

Figure 5.13: Instantaneous lift coefficient $\left(C_{l}\right)$ versus nondimensional $(\tau)$ when $\mathrm{St}=0.16$ and $U_{0}=0:(-), \operatorname{Re}=50 ;(+), \operatorname{Re}=100$.

forces (increased Reynolds number) affect the lift coefficients more in specific intervals of the plunging motion, based on the governing Re. Moreover, comparing the difference between $C_{l}$ of $\operatorname{Re}=200$ and 500 at $\tau=0.15$ and 0.35 shows that although the airfoil is at the same location at these two nondimensional times, the discrepancy is much higher at $\tau=0.15$.

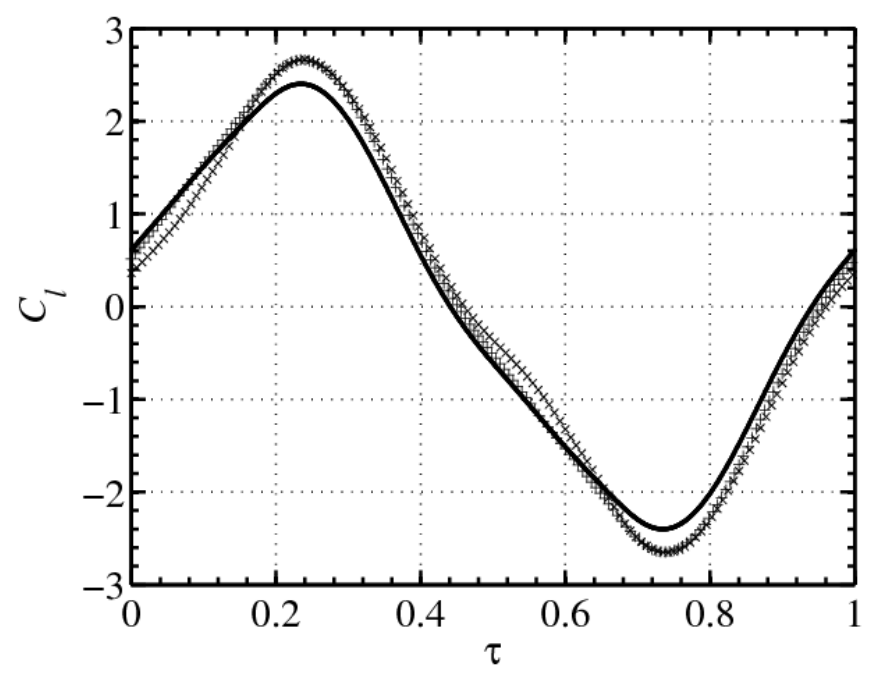

Figure 5.14: Instantaneous lift coefficient $\left(C_{l}\right)$ versus nondimensional time $(\tau)$ for St $=0.16$ when $U_{0} \neq 0$ : (-) $\operatorname{Re}=100,(+) \operatorname{Re}=200,(\times) \operatorname{Re}=500$.

Figures 5.15(a-c) show the vortical patterns around the airfoil when $C_{l_{\max }}$ is obtained for 
the considered Re, where the black color shows higher vorticity magnitude. It is observed that the leading edge vortex (LEV) at $\mathrm{Re}=200$ is more deflected towards airfoil surface than that of $\operatorname{Re}=100$, while TEV is not changed noticeably between $\operatorname{Re}=100$ and 200 . This explains the increase in $C_{l_{\max }}$ when Re increases from 100 to 200 . The LEV at $\operatorname{Re}=$ 500 seems to be stronger and more concentrated than that of $\mathrm{Re}=200$. However, TEV is shown to have a higher strength at $\operatorname{Re}=500$, explaining the equality of $C_{l_{\max }}$ between $\operatorname{Re}$ $=200$ and 500.

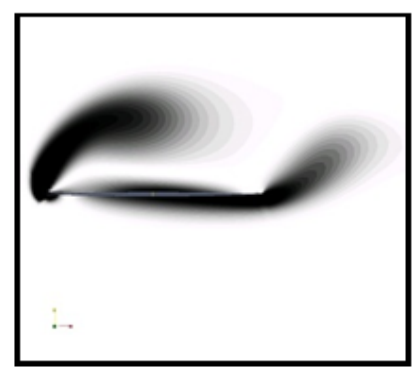

(a)

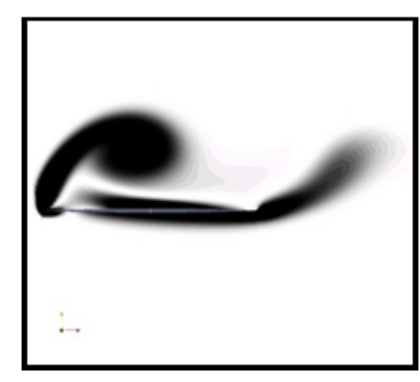

(b)

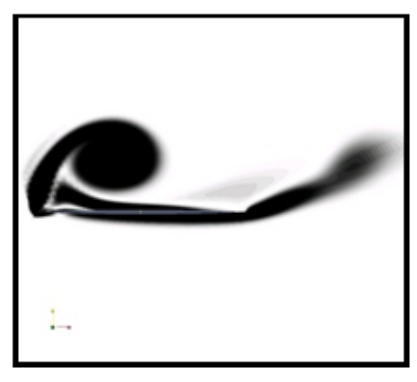

(c)

Figure 5.15: Vortical patterns around the airfoil when maximum lift coefficient $\left(C_{l_{\max }}\right)$ is obtained for $\mathrm{St}=0.16$ and $U_{0} \neq 0$ : (a), $\operatorname{Re}=100$; (b), $\operatorname{Re}=200 ;(\mathrm{c}), \operatorname{Re}=500$.

Figure 5.16 shows the calculated $C_{d}$ versus $\tau$. The maximum drag coefficient $\left(C_{d_{\max }}\right)$ is 0.132, 0.115 and 0.08, at $\operatorname{Re}=100,200$ and 500, respectively, showing that increasing Re decreases $C_{d_{\max }}$. This behavior can be investigated in Figs. 5.17(a-c), presenting the vortical patterns around the airfoil when $C_{d_{\max }}$ is obtained $(\tau \approx 0.52)$. Fig. 5.17(a) shows that there is a LEV mostly concentrated around LE, which sweeps on the airfoil's pressure side by increasing Re, stretches towards TE, but is not separated at any of the considered Re. On the suction side, however, there is a complex vortical pattern; a LEV which is deflected and stays around the TE is strengthened by increasing Re. It seems that there is a secondary circulation region at $\mathrm{Re}=100$ around LE, attached to the airfoil suction side. Increasing Re from 100 to 200 (Fig. 5.17(b)) causes this secondary vortical pattern to detach from the surface, which is eventually strengthened at Re $=500$, Fig. 5.17(c). Moreover, a TEV nonexisting at $\operatorname{Re}=100$, is formed at $\operatorname{Re}=200$ (Figs. 5.17(b, c)). This TEV is also deflected 
towards LE, although there is freestream velocity. This TEV is strengthened at $\operatorname{Re}=500$ and is more interacted with the secondary LEV, Fig. 5.17(c). This complex vortical pattern causes $C_{d_{\max }}$ to decrease by increasing Re.

Another interesting effect of Re is that increasing Re also causes the airfoil to generate thrust. The plunging airfoil only generates drag at $\mathrm{Re}=100$, Fig. 5.16. However, for some intervals of the plunging motion, the airfoil generates thrust at $R e=200$. In fact, for Re $=200$, thrust is produced within $0.15<\tau<0.35$ and $0.65<\tau<0.85$ intervals. It should also be mentioned that $C_{d_{\min }}=0.01$ at $\mathrm{Re}=100$, decreases to $C_{d_{\min }}=-0.04$ (thrust) at $\operatorname{Re}=200$. Increasing Re from 200 to 500 decreases the magnitude of $C_{d_{\min }}$, however, the airfoil generates thrust for a longer period of time; $0.12<\tau<0.36$ and $0.62<\tau<0.86$. Moreover, the first $C_{d_{\min }}$ is obtained with lead at $\tau \approx 0.28,0.25$ and 0.23 , for $\operatorname{Re}=100,200$ and 500, respectively, whereas, the calculated $C_{d_{\max }}$ does not show a significant lead or lag, compared to those observed for $C_{d_{m i n}}$.

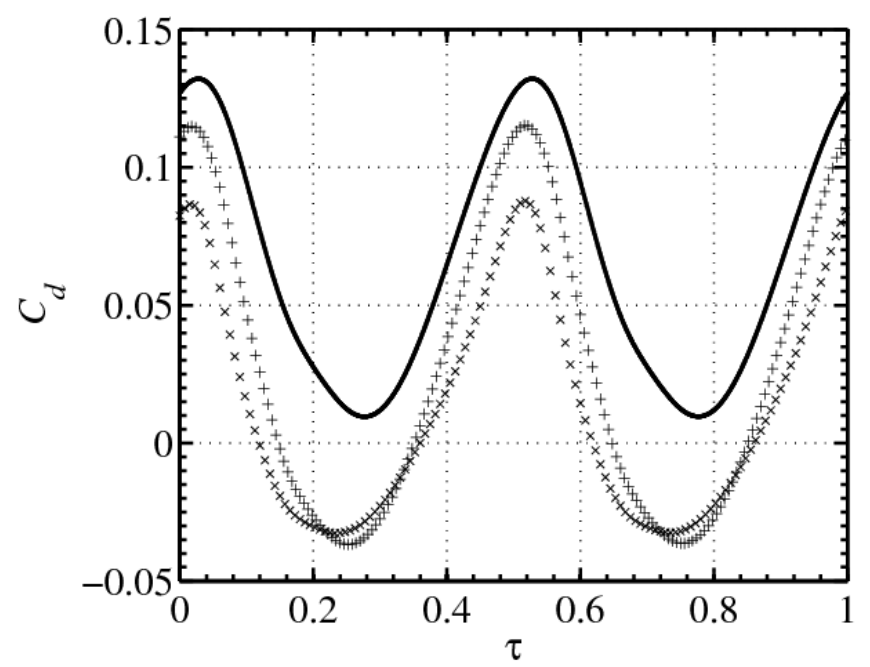

Figure 5.16: Instantaneous drag coefficient $\left(C_{d}\right)$ versus nondimensional time $(\tau)$ for $\mathrm{St}=$ 0.16 when $U_{0} \neq 0$ : (-) $\operatorname{Re}=100,(+) \operatorname{Re}=200,(\times) \operatorname{Re}=500$.

The pitching moment coefficient $\left(C_{m}\right)$ is an important parameter from control and stability perspectives. The effect of Re on $C_{m}$ versus $\tau$ is shown in Fig. 5.18. It is observed that the airfoil faces negative $C_{m}$ for $\tau<0.4$ and positive $C_{m}$ for $0.4<\tau$. Increasing Re 


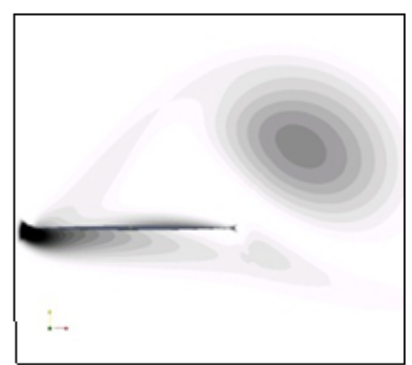

(a)

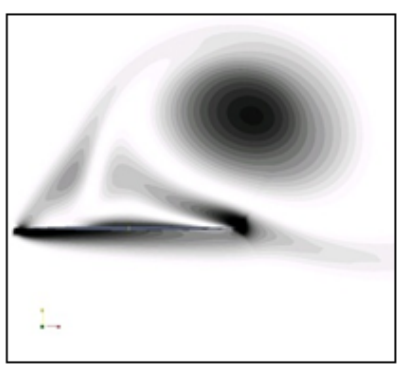

(b)

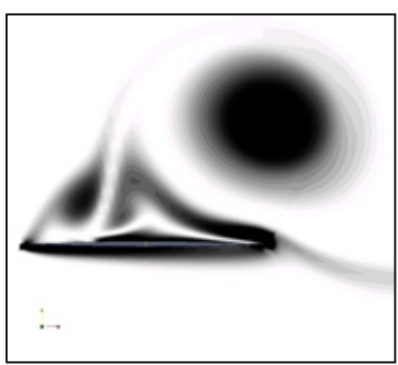

(c)

Figure 5.17: Vortical patterns around the airfoil when maximum drag coefficient $\left(C_{d_{\max }}\right)$ at $\tau=0.52$ is obtained for $\mathrm{St}=0.16$ and $U_{0} \neq 0$ : (a), $\operatorname{Re}=100 ;(\mathrm{b}), \operatorname{Re}=200 ;(\mathrm{c}), \operatorname{Re}=500$.

increases $\left|C_{m}\right|$ for $\tau<0.4$. For example, $\left|C_{m_{\max }}\right|$ increases when Re increases, where the corresponding vortical patterns are shown in Figs. 5.19(a-c). It should be mentioned that as $C_{m_{\max }}$ between $0<\tau<0.4$ is negative, it could be concluded that the influence of counter clockwise circulation of the TEV on the calculated $C_{m}$ is greater than that of the LEV. Re has an inverses effect on $C_{m}$ when $0.4<\tau<1$, with the exception of $200<\operatorname{Re}<500$ and $0.66<\tau<0.8$.

The vortical patterns are investigated at $\tau=0.6$ in Figs. 5.19(a-c). Fig. 5.19(a) shows a LEV attached to LE and stretched towards TE. Increasing Re causes this vortex to approach the airfoil surface and to rotate around the $\mathrm{TE}$ at $\mathrm{Re}=200$. Increasing Re further to 500 causes this vortex to be closer to the surface with a higher strength. Moreover, increasing Re strengthens the TEV. In addition, the flow on the suction side of the airfoil at $\operatorname{Re}=100$ starts to separate at $\operatorname{Re}=200$, which eventually forms a secondary vortical pattern at Re $=500$. Therefore, the decreasing effect of Re on $C_{m}$ is accompanied with the very complex vortical behavior, as shown in Figs. 5.19(a-c).

Finally, the changes in the average $\bar{C}_{l}$ and $\bar{C}_{d}$ are studied and listed in Table 5.3. As it can be seen, the magnitude of $\left|\bar{C}_{l}\right|$ does not change significantly, but $\left|\bar{C}_{d}\right|$ decreases noticeably, resulting in increased $\left|\frac{\bar{C}_{l}}{\bar{C}_{d}}\right|$. 


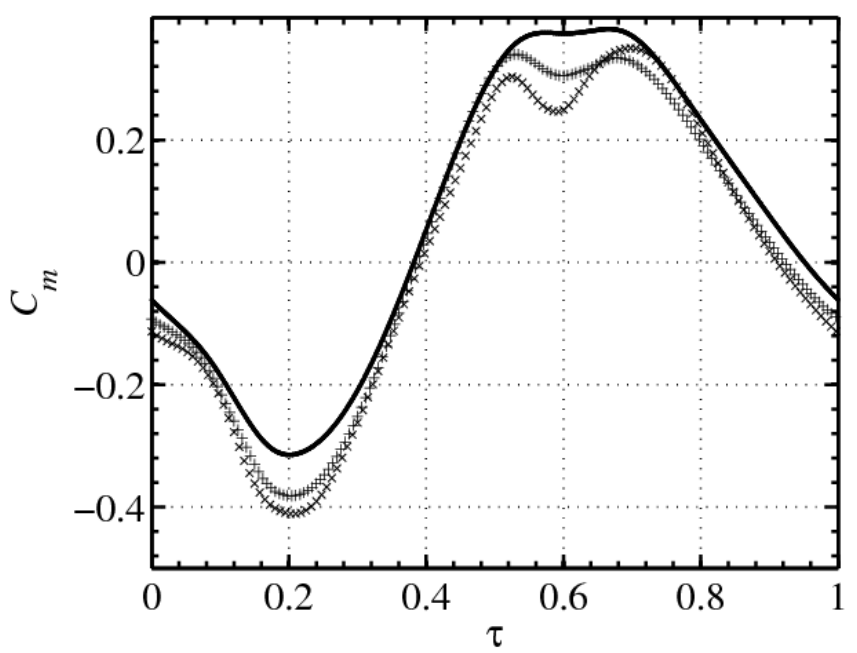

Figure 5.18: Instantaneous pitching moment coefficient $\left(C_{m}\right)$ versus nondimensional time $(\tau)$ for $\mathrm{St}=0.16$ when $U_{0} \neq 0:(-) \operatorname{Re}=100,(+) \operatorname{Re}=200,(\times) \operatorname{Re}=500$.

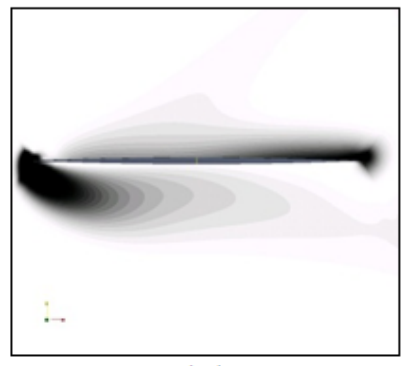

(a)

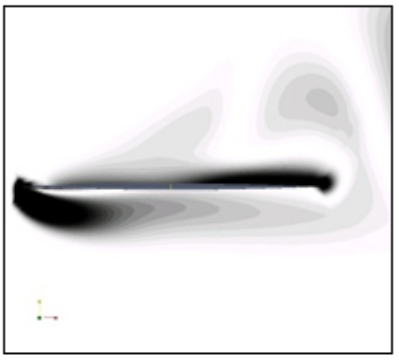

(b)

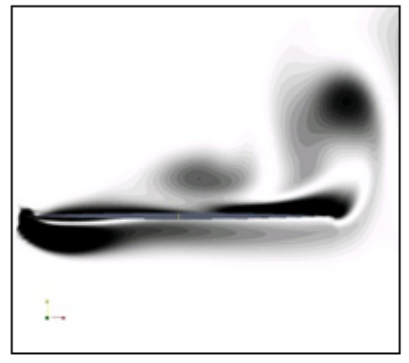

(c)

Figure 5.19: Vortical patterns around the airfoil when minimum moment coefficient $\left(C_{m_{\min }}\right)$ at $\tau=0.6$ is obtained for $\mathrm{St}=0.16$ and $U_{0} \neq 0$ : (a), $\mathrm{Re}=100 ;(\mathrm{b}), \operatorname{Re}=200 ;(\mathrm{c}), \operatorname{Re}=$ 500 , the vertical position is the same for all figures.

\subsection{Summary and conclusion}

Simulations of a 2-D plunging airfoil in LRN flow regime are carried out based on FVM and N-S equations. The computations are conducted for both zero and nonzero freestream velocities. The effects of Re and St on the lift, drag, and moment coefficients are investigated and the details of flow patterns and vortical structures are examined. In the simulations of the effects of St without freestream velocity, it is found that higher St increases the magnitude of the lift coefficients with significant lead/lag induced into the instantaneous lift coefficients. The lead and lag in the location of the peak lift coefficients are found to be 
Table 5.3: Mean lift and drag coefficients $\left(\bar{C}_{l}\right.$ and $\left.\bar{C}_{d}\right)$ for the investigated Re when $U_{0} \neq 0$

\begin{tabular}{ccc}
\hline \hline Re & Upstroke & Downstroke \\
\hline 100 & $\bar{C}_{l}=1.304$ and $\bar{C}_{d}=0.065$ & $\bar{C}_{l}=1.303$ and $\bar{C}_{d}=0.065$ \\
200 & $\bar{C}_{l}=1.439$ and $\bar{C}_{d}=0.028$ & $\bar{C}_{l}=1.434$ and $\bar{C}_{d}=0.028$ \\
500 & $\bar{C}_{l}=1.411$ and $\bar{C}_{d}=0.013$ & $\bar{C}_{l}=1.409$ and $\bar{C}_{d}=0.013$ \\
\hline
\end{tabular}

negligible in the simulations of St effect with freestream velocity compared to those without freestream. Moreover, including the freestream in the simulation, a secondary peak of lift coefficient is observed at the highest St, followed by the lower minimum peak lift coefficient than the maximum lift coefficient, where maximum and minimum lift coefficients are almost equal for other Strouhal numbers for both zero and non-zero freestream velocity simulations. Moreover, the non-zero freestream velocity simulations show that drag coefficient decreases with increasing St, where eventually the airfoil generates thrust at some time intervals during its plunging motion with the expense of a higher maximum drag coefficient. In addition, the airfoil encounters a negative pitching moment for a longer time interval when St increases in non-zero oncoming flow cases. The aerodynamic performance also increases with increasing St.

Reynolds number increases the lift coefficient mostly around its peak values. However, the non-zero oncoming flow simulations show that Re does not change the peak lift coefficients significantly for the range of Re considered in this study. The drag coefficients is found to decrease when Re increases, and the initially drag producing airfoil, generates thrust for some time intervals during the motion. Further increase of Re causes the airfoil to generate thrust for a longer period, penalized by a slightly higher drag coefficient (lower thrust coefficient). Unlike the effects of St on the pitching moment, Re does not change the time interval for which negative or positive pitching moment is exerted on the airfoil, but Re changes the peaks of the pitching moment coefficient. Moreover, Re increases the aerodynamic performance in the non-zero oncoming velocity computations.

The conclusions drawn from this chapter show that most the mentioned effects of St and 
Re are the direct results of LEV and TEV patterns, their interactions and secondary vortical patterns generated during the plunging motion, well explaining the need for more studies on the aerodynamics of oscillating airfoil/wings in LRN flow regime. In the next chapter, the flow field characteristics of a figure-of-eight-like flapping airfoil are investigated. 


\section{Chapter 6}

\section{Flow field characteristics of a figure-of-eight-like flapping airfoil}

The flow field of a thin ellipsoidal geometry performing a modified figure-of-eight-like flapping pattern is simulated, and vortical patterns around the airfoil are examined in detail. The effects of different unsteady flow and system parameters on the flow characteristics are also explored. The investigated parameters are namely the amplitude of pitching oscillations, phase angle between pitching and plunging motions, mean angle of attack, Reynolds number (Re) and Strouhal number (St) based on the translational amplitudes of oscillations and the pitching axis location $(x / c)$. It is shown that these parameters change the instantaneous force coefficients both quantitatively and qualitatively. It is also observed that the strength, interaction, and convection of the vortical structures surrounding the airfoil are significantly affected by the variations of these parameters.

\subsection{Introduction}

Flapping, in the form of both forced and flow-induced oscillations, is the most observed means of force generation in different LRN applications, such as MAVs. Various kinematic patterns simulating LRN flapping motion are reported in the literature, which are meant to 
improve efficiency and/or thrust and lift and contribute to the understanding of the respective phenomena. Yet, more studies are needed to obtain a better insight into the subject matter. Flapping flow field characteristics depend on the governing kinematics and parameters. Ellington [7] stated that the predicted unsteady forces by classical analytical methods are insufficient and unreliable for flapping motions. The importance of the corresponding viscous flow phenomena, LE/TE vortical patterns and their interaction, which are not considered in the analytical solutions, makes these methods inappropriate for LRN flapping studies. Several experimental studies have shown the importance of the LRN viscous phenomena in a flapping motion force generation. Experimental techniques can provide the most accurate results, but they are rather expensive and time consuming.

Freymuth's experiments [55] on a flapping airfoil in its thrust generating regime showed that dynamic stall leads to high thrust coefficients. The importance of dynamic stall, rotational effect and wake-capturing mechanisms on an insect flapping wing was investigated by Nagai et al. [56]. They illustrated that these mechanisms act differently during upand down-strokes, and concluded that lift is mainly generated during downstroke while a higher thrust is achieved during upstroke. Three-dimensional (3-D) investigation of a flapping wing by Isaac et al. [57] showed that an optimum flapping frequency might exist that gives the highest performance. The 3-D harmonic flapping wing experiments of Wang et al. [58] demonstrated that the unsteady force coefficients weakly depend on the amplitude of oscillations but are strongly affected by the phase difference between pitching and plunging portions of the flapping motion. Singh and Chopra [59] investigated different shapes of hovering insect-based flapping wings in hover. They observed that the amplitude and frequency of the pitching oscillations are of great importance to the thrust generation.

CFD is also widely used in LRN flapping studies. Lee et al. [84] investigated the role of LEVs/TEVs in force generation and concluded that LEVs affect thrust beside their effect on increasing the lift. The two dimensional (2-D) figure-of-eight-like motion simulations of Lee et al. [86] showed that lift is mainly produced during downstrokes, while thrust is 
mostly achieved at the end of upstrokes. They attributed the generated thrust to the vortex pairing, which is different from the conventional inverse Karman vortex street found in sinusoidal flapping motions. Mittal et al. [85] numerically simulated a flapping airfoil flow field and concluded that the formation of an inverse Karman vortex street is accompanied with the highest thrust efficiency. Tang et al. [87] simulated a hovering elliptic airfoil using N-S equations and investigated the effects of Re, reduced frequency and flapping kinematics on the flow field. They also confirmed the significance of the delayed stall mechanism on the generated forces and observed the existence of the wake-capturing mechanism in their simulations. Bos et al. [88] studied different flapping kinematics and compared their aerodynamic performance numerically. The system parameters such as plunging amplitude and frequency of oscillations were shown by Kinsey and Dumas [89] to have the highest impact on the instantaneous fluid forces. They noticed that higher lift is produced during advanced rotations, where the pitching oscillation starts before the end of each plunging stroke. Kaya and Tuncer [90] studied a nonsinusoidal flapping pattern and performed an optimization study on the kinematics to obtain the maximum thrust and/or propulsive efficiency. In their 2-D numerical simulation, they observed that the maximum thrust coefficient can be obtained if the airfoil stays at almost constant angle of attack during the up and down strokes, and the pitching occurs at the end of each stroke. However this is achieved at very high acceleration rates in both pitch and plunge, and at the expense of the propulsive efficiency. Sun and Tang [91] simulated a 3-D hovering wing using N-S equations and examined advanced, symmetrical and delayed rotation cases. Young et al. [92] conducted simulations of a 3-D flapping wing and performed a parametric study. They concluded that both lift and thrust are influenced by the frequency of oscillations. In another study, Dong et al. [93] explored the wake topology of a finite aspect ratio wing performing a harmonic flapping motion and reconfirmed that the topology is different from those of infinitely long wings. They also studied the effects of Re and St on the wing's performance and investigated their importance to the unsteady forces. Ramamurti and Sandberg [94] performed 3-D Finite Element 
Method (FEM) based simulations of flapping motion and studied the effect of the phase angle between the rotational and translational motions on the instantaneous forces. They observed that the advanced rotations yield higher thrust coefficients than the symmetrical and delayed cases. Ramamurti and Sandberg [95] also investigated 3-D flapping motions under different conditions such as gust loading and flexibility and compared the lift and thrust coefficients as the performance benchmarks.

The present study is conducted to provide a better understanding of the effective parameters on the fluid dynamics of a flapping airfoil under a figure-eight-like kinematics in LRN regime. Figure-of-eight patterns are not investigated extensively in the literature, and the reported studies are based on almost horizontally-flat pattern figure-of-eight kinematics. The kinematics considered in the present investigation has very inclined up/down strokes. As it was also shown by Wang [140], the inclined patterns have substantial drag forces, which contribute to the required hovering force. Some patterns reported in the literature have constant angle of attack during their strokes and the pitching oscillations occur at the end of each stroke, while the pitching oscillations of the present kinematics are constantly performed during a whole cycle. Moreover, in the present research, an extensive parametric study is conducted, while most of the investigations reported in the literature examine the effects of a few parameters on the flapping fluid dynamics. The investigated range of Re is chosen based on the typical range of applications such as MAVs and fish-like robots. The flow field is simulated using CFD with unsteady, incompressible N-S equations and FVM, and the variations of lift, drag and pitching moment coefficients under different operating conditions are explored in detail.

\subsection{Numerical simulation method}

The flow field of a 2-D flapping airfoil in LRN regime is governed by the continuity and $x$ and $y$ direction momentum equations. The computational domain is discretized using an 
O-type mesh. The far-field boundary with symmetry condition is set to 30c to minimize its undesired effects on the airfoil's surrounding flow field. The airfoil surface is set to a no-slip boundary condition. The computations utilize $50 \times 10^{3}$ cells and 2000 time steps per period. The grid has 8800 (176 tangentially $\times 50$ radially) cells within one chord-length from the airfoil surface, and is graded radially and tangentially (from LE/TE to the mid-chord). The radial grading is such that the distance between the center of the first layer and the airfoil surface is $0.002 c$. This choice was made after extensive grid and time step independence analyses. Fig. 6.1 shows the schematic of the airfoil and the computational domain and Fig. 6.2 shows the schematic of the O-type grid used around the airfoil.

FVM is used to investigate the fluid dynamics of an ellipsoidal airfoil with $2 \%$ thick-

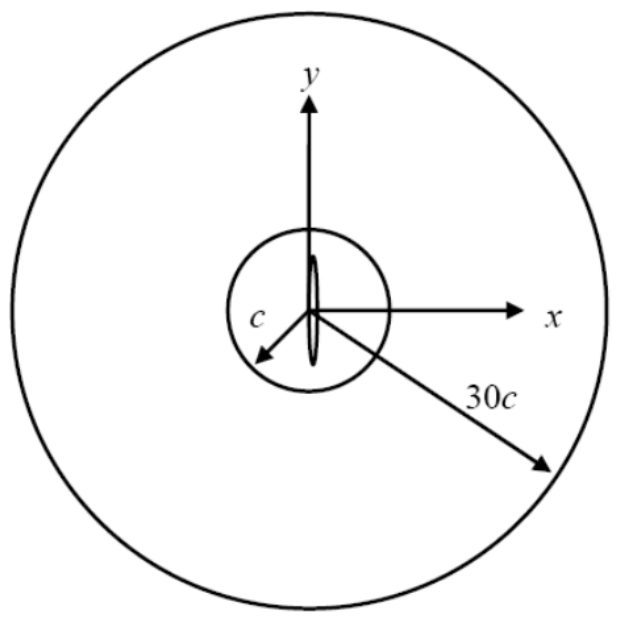

Figure 6.1: Schematic of the airfoil and the computational domain.

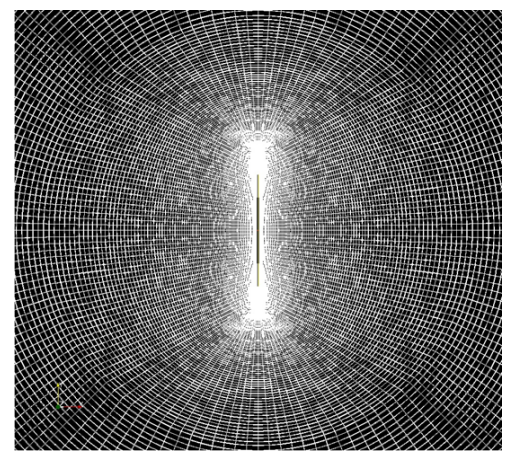

Figure 6.2: Schematic of the O-type grid around the airfoil. 
ness. The computations utilize a second order central differencing scheme for the convective and diffusive terms and a second order Euler implicit scheme for temporal discretizations. The resulting linear system of equations is treated with a Preconditioned Conjugate Gradient (PCG) solver, and the Semi-Implicit Method for Pressure Linked Equations (SIMPLE) algorithm is used for the pressure-velocity coupling. The computations are carried out in OpenFOAM ${ }^{\circledR}$, and the motion is based on the Laplace mesh motion algorithm [107].

\subsection{Kinematics and equations of motion}

The present flapping pattern is a figure-of-eight-like kinematics [5] obtained from a pair of counter rotating-flapping blade configuration, Fig. 6.3 .

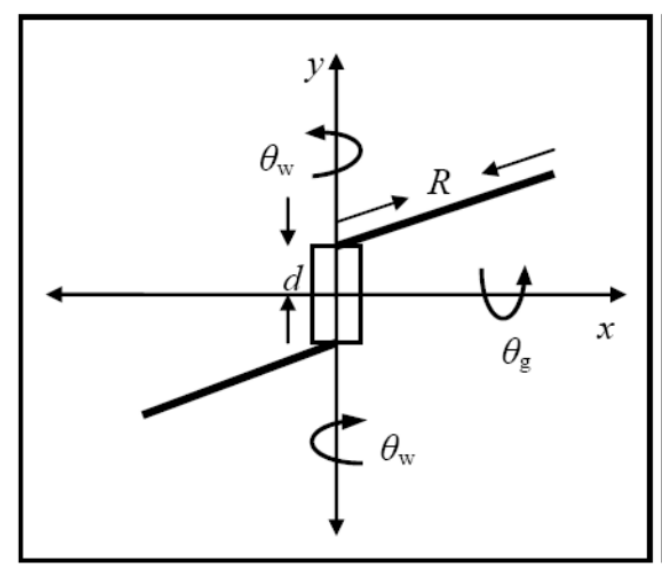

Figure 6.3: The schematic of the pair of rotating-flapping blade configuration.

As it can be seen in Fig. 6.3, two blades with span $R$ and distance $d$ from the centerline are connected to a shaft. The blades counter-rotate about the $y$ axis $\left(\theta_{w}\right)$. The shaft rotates about the $x$ axis $\left(\theta_{g}\right)$ and its motion is transferred to the blades causing the simultaneous flapping and rotation of the blades in opposite directions with respect to each other. Using an appropriate rotation matrix, the $3-\mathrm{D}$ equations of motion can be found in spherical coordinate system. Specifically, the following equations are obtained for the mid-chord position of each 
blade tip cross section:

$$
X_{M C}=\left(\begin{array}{c}
R \sin (\theta) \\
d \cos (\phi)-R \sin (\phi) \cos (\theta) \\
d \sin (\phi)+R \cos (\phi) \cos (\theta)
\end{array}\right)
$$

where $X_{M C}$ is the mid-chord position vector, $\theta=\theta_{w}=-\omega t$ and $\phi=\theta_{g}=\omega t$ (where $\omega$ is the angular velocity).

Using 2-D $X_{M C}$ along with the respective pitching oscillations, the present figure-ofeight-like kinematics is given by:

$$
\begin{aligned}
& x(t)=-R \sin (2 \pi f t), \\
& y(t)=d \cos (2 \pi f t)-\frac{R}{2} \sin (4 \pi f t), \\
& \alpha(t)=\alpha_{0}-\bar{\alpha} \sin (2 \pi f t+\phi),
\end{aligned}
$$

where $x(t)$ and $y(t)$ indicate the horizontal and vertical positions of the airfoil mid-chord, respectively, $\alpha(t)$ is the instantaneous angle of attack, $d$ and $R$ stand for the translational amplitudes of oscillations (Figs. 6.3 and 6.4), and $\bar{\alpha}, \alpha_{0}, \phi$ and $f$ indicate the amplitude of pitching oscillations, mean angle of attack, phase lag/lead, and the frequency of oscillations, respectively. Figure 6.4 shows the figure-of-eight pattern obtained from Equations 6.2.

As it was mentioned earlier in this chapter, most of the hovering kinematic patterns available in the literature are elliptical, non-crossing parabolic-like or almost horizontal figureof-eight patterns [5], whereas an asymmetrical figure-of-eight flapping kinematics is used in this study. In the horizontally flat patterns, the vertical motion is negligible compared to the horizontal portion; hence, it does not contribute to the force generation significantly. However, in this figure-of-eight the vertical motion plays a substantial role in the generation of the unsteady forces, Fig. 6.4. This feature of the model allows for the contribution of lift and drag forces in vertical lift, resulting in more efficient upstrokes [5,140]. Another aspect 


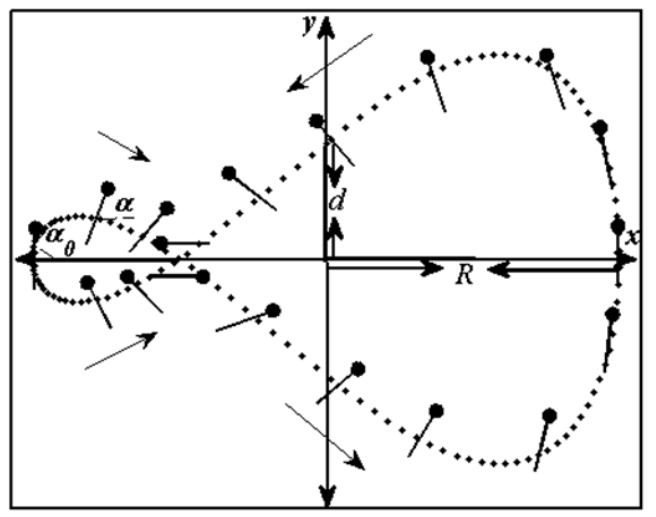

Figure 6.4: The 2-D kinematic pattern obtained from Equations 6.2.

of this kinematics is the simultaneous rotation and significant vertical translation at the end of each stroke, which could the vertical lift generation.

\subsection{Reference velocity, force and moment coefficients calculations}

The reference velocity in the computations of Re and St is based on the average velocity during one period of figure-of-eight-like flapping oscillations [88]. This choice is made as the flapping simulations are performed in a stationary fluid, and it is needed for the calculation of Re, St, $C_{l}, C_{d}$ and $C_{m}$. Thus, $U_{r e f}$ is defined by the following equation:

$$
U_{\text {ref }}=\frac{1}{T} \int_{0}^{T} \sqrt{u^{2}+v^{2}} d t
$$

where $u$ and $v$ are the mid-chord horizontal and vertical velocity components, respectively, and $T$ stands for the period of oscillations.

A reference velocity (different from the one used for the nondimensionalization of N-S equations) is also required for calculation of the unsteady force and moment coefficients. The 
lift $\left(C_{l}\right)$, drag $\left(C_{d}\right)$, and moment $\left(C_{m}\right)$ coefficients are obtained from the following equations:

$$
C_{l}=\frac{F_{y}}{\bar{q} S} ; \quad C_{d}=\frac{F_{x}}{\bar{q} S} ; \quad C_{m}=\frac{M}{\bar{q} S c},
$$

where $F_{y}$ and $F_{x}$ are the forces in $y$ (lift) and $x$ (drag) directions, respectively, and $M$ stands for the pitching moment about the airfoil mid-chord (Fig. 6.5), $S=c \times b$, where $c$ is the chord-length and $b=1$, and $\bar{q}$ stands for the mean dynamic pressure defined as:

$$
\bar{q}=\frac{1}{2} \rho \bar{U}^{2}=\frac{1}{2} \rho \frac{1}{T} \int_{0}^{T}\left(\left(\frac{\partial x(t)}{\partial t}\right)^{2}+\left(\frac{\partial y(t)}{\partial t}\right)^{2}\right) d t,
$$

where $\rho$ is the fluid density.

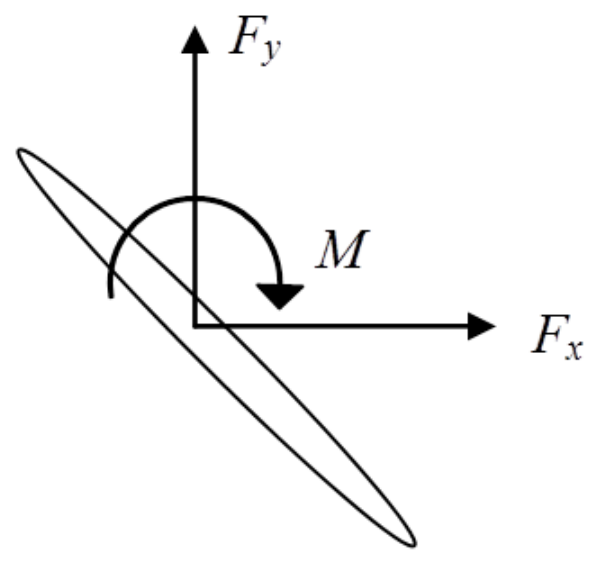

Figure 6.5: Forces and moment on the airfoil.

\subsection{Evaluation of the results}

Several simulations are performed to obtain the required grid and time step resolutions and to validate the results using the data available in the literature. The fluid dynamics of an ellipse performing a flapping motion at $\mathrm{Re}=75$, also studied by Wang et al. [58] and Bos et al. [88], is first simulated for various mesh and time step resolutions. The motion is governed 
by the following equations:

$$
\begin{aligned}
& x(t)=\frac{A_{0}}{2} \cos (2 \pi f t), \\
& \alpha(t)=\alpha_{0}+\beta \sin (2 \pi f t+\phi),
\end{aligned}
$$

with $A_{0}=2.8, f=0.25, \alpha_{0}=\pi / 2, \beta=\pi / 4$ and $\phi=0$.

Four mesh sizes of $12.5 \times 10^{3}, 25 \times 10^{3}, 50 \times 10^{3}$ and $100 \times 10^{3}$ cells with 2000 time steps in each flapping period, are examined. As shown in Fig. 6.6, the difference between the $C_{l}$ calculated from $50 \times 10^{3}$ and $100 \times 10^{3}$ cell models is negligible. Hence, the mesh of $50 \times 10^{3}$ cells is adequately refined to yield accurate solutions.

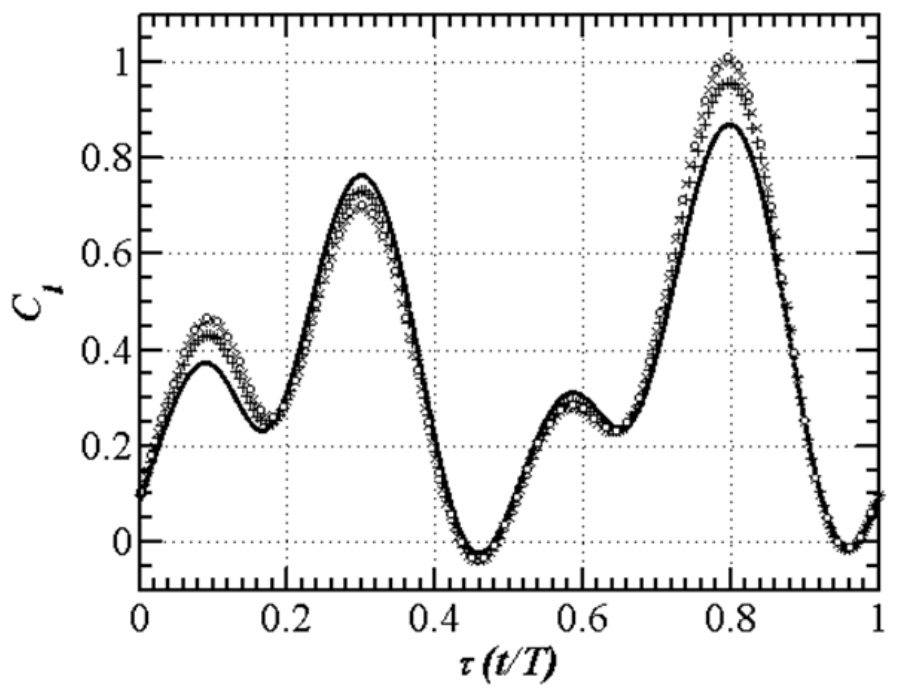

Figure 6.6: Instantaneous lift coefficient $\left(C_{l}\right)$ versus nondimensional time $(\tau)$ for flapping motion of Equations 6.6 and different grid sizes: (-), $12.5 \times 10^{3} ;(+), 25 \times 10^{3} ; \times, 50 \times 10^{3}$; (o), $100 \times 10^{3}$.

In $50 \times 10^{3}$ cells model, 176 and 284 cells are used in tangential and radial directions, respectively. The mesh refinement is done in both tangential and radial directions simultaneously. Moreover, $176 \times 50$ cells are considered within one-chord length from the airfoil surface to resolve the generated vortical patterns accurately. After obtaining a grid indepen- 
dent solution, the temporal resolution is also varied for 500, 1000 and 2000 time steps within a period. Figure 6.7 shows that 2000 time steps are sufficient for a temporally independent solution. Therefore, the simulation with $50 \times 10^{3}$ cells and 2000 time steps is found to yield reasonably accurate solutions.

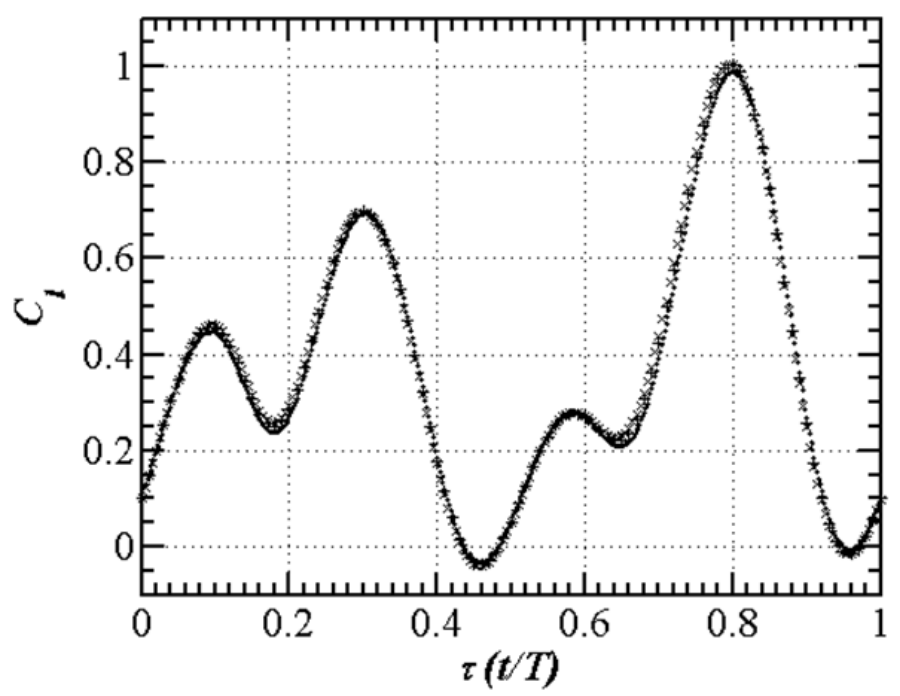

Figure 6.7: Instantaneous lift coefficient $\left(C_{l}\right)$ versus nondimensional time $(\tau)$ for flapping motion of Equations 6.6 and different time steps: $(-), 500 ;(+), 1000 ;(\times), 2000$.

Finally, the calculated $C_{l}$ of the flapping motion governed by Equations 6.6 is compared with those reported by Wang et al. [58] and Bos et al. [88]. A thin (thickness of 2\%) ellipsoidal airfoil similar to those used in Bos et al. [88] and Wang et al. [58] is used. Figure 6.8 shows that the computed $C_{l}$ is in good agreement (less than $5 \%$ difference in the mean lift coefficient) with the results available in the literature. The small discrepancy can be due to the different LE/TE shapes of the ellipse models, or different schemes used for the discretization of the spatial terms in N-S equations. Wang et al. [58] used a 4th order finite difference method and stream-vorticity function form of N-S equations. However, the 2nd order finite volume method with N-S equations are used in the present study.

An extensive parametric study is conducted on a thin $(t / c=0.02)$ ellipsoidal airfoil flapping according to Equations 6.2. The effects of $\alpha_{0}, \bar{\alpha}, \phi$, St $(R$ and $d)$, Re and $x / c$ on $C_{l}, C_{d}, C_{m}$ and the vortical patterns surrounding the airfoil are investigated. The base 


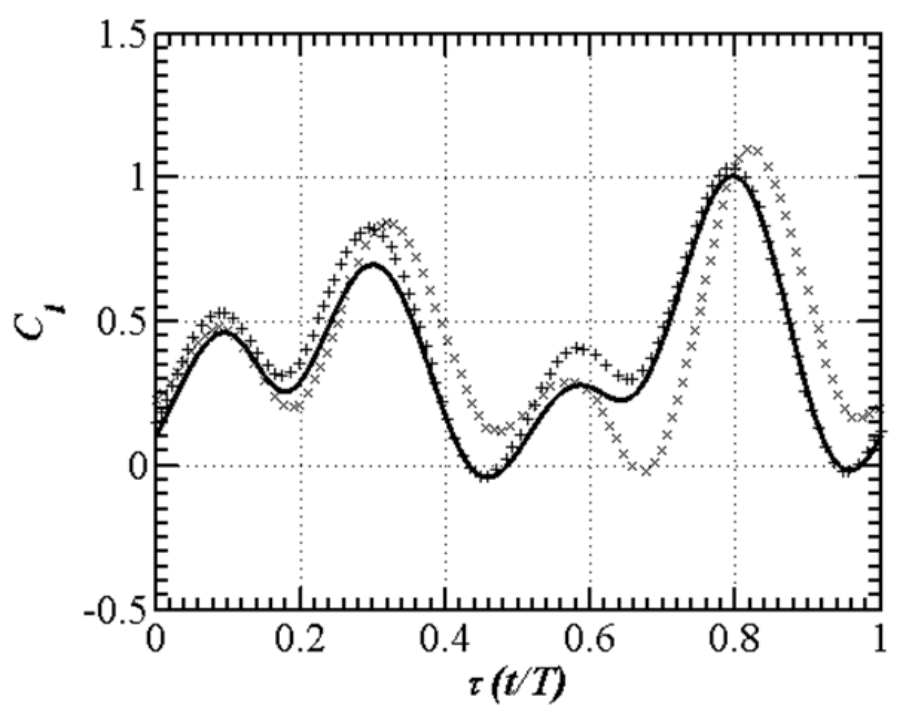

Figure 6.8: Instantaneous lift coefficient $\left(C_{l}\right)$ versus nondimensional time $(\tau)$ of for the flapping motion of Equations 6.6: (-), present simulation; (+), Bos et al. [88]; (×), Wang et al. [58].

case of the present study is when $R=1.4 c, d=0.7 c, f=0.25, \alpha_{0}=90^{\circ}, \bar{\alpha}=45^{\circ}, \phi=$ $0, x / c=0.5$ and $\operatorname{Re}=75$. The parametric studies are performed by changing one of the variables while keeping the others fixed. The obtained vortical patterns for the base case are shown in Figs. 6.9(a) through 6.9(i), where (a-i) are shown on the kinematics in Fig. 6.10. Figure 6.9(a) corresponds to $\tau=0.04$ when the airfoil is almost at the beginning of the figure-of-eight motion. As can be seen, two LEVs and one TEV are attached to the left and right sides of the model, respectively. Figure 6.9(b) shows the flow contours at $\tau=0.1$, where the LEV on the right side of the ellipse is mostly detached from the surface but not from the LE. This explains the increase in $C_{l}$ (Fig. 6.11) despite having the mostly detached LEV from the surface. Figure 6.9(c) shows that the previous LEV is separated, but another LEV is formed. A TEV is also generated which could explain the increase in $C_{d}$, Fig. 6.13. Figure $6.9(\mathrm{~d})$ shows the vorticity contour at $\tau=0.25$, when the model is at the maximum pitch (midstroke); hence, $C_{l_{\max }}$ and $C_{d_{\max }}$ are achieved (refer to Figs. 6.10 and 6.13). At this instant, both LEV and TEV are strong vortices and attached to the surface. After the midstroke, both $C_{l}$ and $C_{d}$ start to decrease, which is due to the full separation of LEV and 
mostly separated TEV. Figure 6.9(f) shows the contours after the first half cycle. It can be seen that a LEV and a TEV are formed and the airfoil faces the previously separated vortices, the so-called wake capturing mechanism, which causes the increase in $C_{l}$ and $C_{d}$, Figs. 6.11 and 6.13. The second peaks in the lift and drag coefficients are higher than the first ones, which could be related to the wake-capturing mechanism observed in Figure 6.9(g). The drop seen in Figure 6.11 corresponds to the separation of the LEV and TEV seen in Figure 6.9(h). After this separation, two LEVs are formed, which result in the minor peak in $C_{l}$, Fig. 6.11 .

\subsubsection{Effect of the amplitude of pitching oscillations}

The effect of the amplitude of pitching oscillations on the airfoil's flow field is investigated for $\bar{\alpha}=15^{\circ}, 30^{\circ}, 45^{\circ}$ and $60^{\circ}$. Figures 6.11 through 6.15 present the calculated $C_{l}$, first and second $C_{l_{\max }}$ corresponding vortical patterns, $C_{d}$ and $C_{m}$, respectively, revealing the effects of $\bar{\alpha}$ on the airfoil's fluid dynamics characteristics. It is found, based on the following

discussions, that the higher $\bar{\alpha}$ improves the aerodynamic performance $\frac{\bar{C}_{l}}{\bar{C}_{d}}$ by increasing $\bar{C}_{l}$ but decreasing $\bar{C}_{d}$ at the same time. It should be mentioned that the mean lift coefficient $\left(\bar{C}_{l}\right)$ is averaged over a complete flapping cycle, but $\bar{C}_{d}$ is the average between the two half strokes as the airfoil changes its direction at $\tau=0.5$.

As shown in Fig. 6.11, there are two major maximum lift coefficients $\left(C_{l_{\max }}\right)$. The first $C_{l_{\max }}$ increases at higher values. The percentage increase of $C_{l_{\max }}$ is almost $133 \%$ between $\bar{\alpha}$ $=15^{\circ}$ and $30^{\circ}, 45 \%$ between $\bar{\alpha}=30^{\circ}$ and $45^{\circ}$, and $17 \%$ between $\bar{\alpha}=45^{\circ}$ and $60^{\circ}$. Therefore, it seems that at higher $\bar{\alpha}$, increasing $\bar{\alpha}$ has less increasing effect on the first $C_{l_{\max }}$. This trend can be explained by investigating the generated vortical patterns around the airfoil.

Figures 6.12(a) through 6.12(d) show the vortices around the airfoil when the first major $C_{l_{\max }}$ is obtained. As seen in these figures, the LEVs are attached to the LE. The size of the LEV core (indicated by the black colour) is increased at higher amplitudes of pitching oscillations $\bar{\alpha}$, meaning that a stronger LEV is formed. Moreover, the LEVs are closer to 


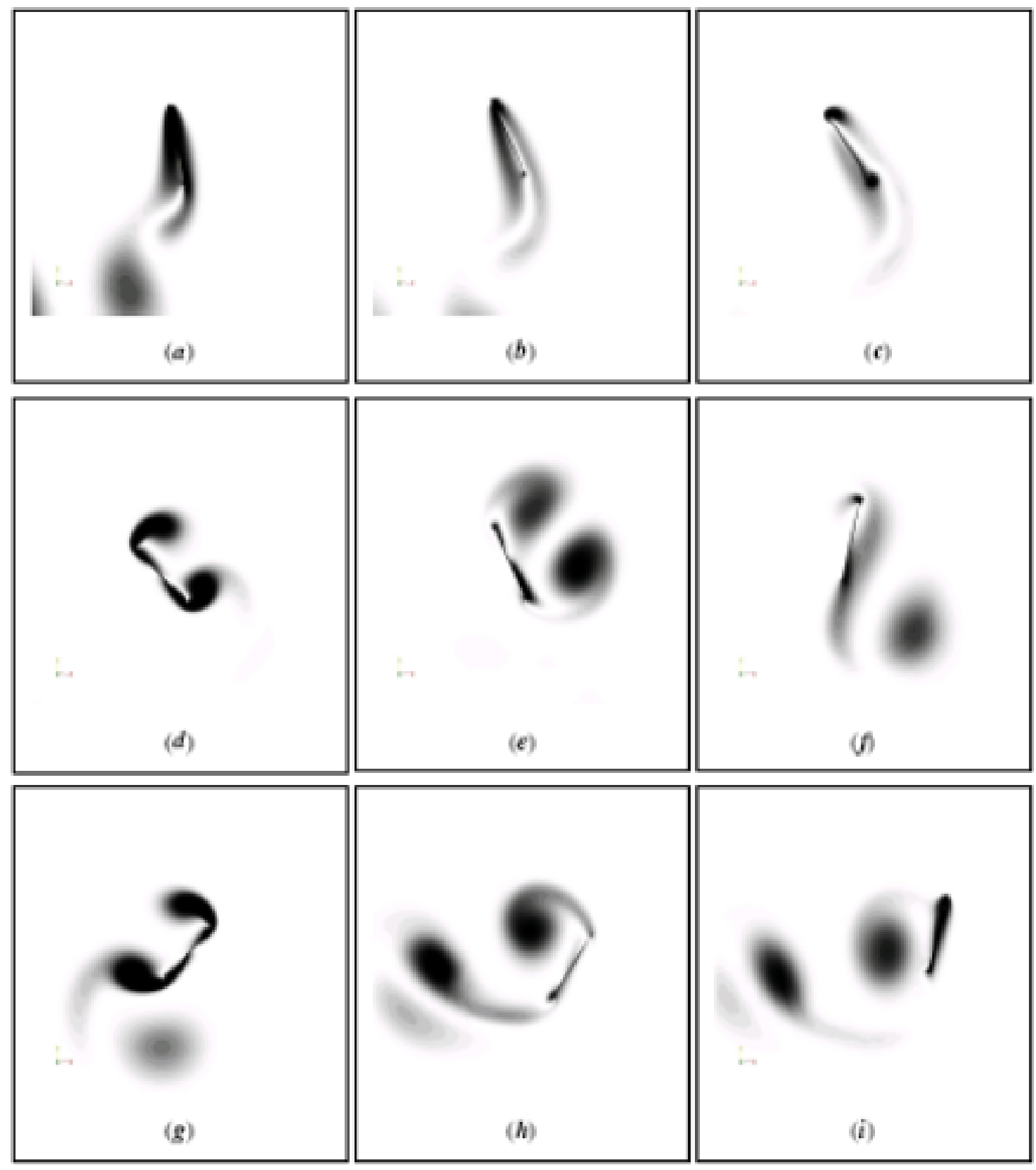

Figure 6.9: Vortical patterns around the airfoil for $R=1.4 c, d=0.7 c, f=0.25, \alpha_{0}=90^{\circ}$, $\phi=0, \operatorname{Re}=75, \bar{\alpha}=45^{\circ}:$ (a) $\tau=0.04$; (b) $\tau=0.1$; (c) $\tau=0.16$; (d) $\tau=0.24$; (e) $\tau=0.4$; (f) $\tau=0.54$; (g) $\tau=0.72$; (h) $\tau=0.86$; (i) $\tau=0.94$.

the airfoil surface and less convected at higher $\bar{\alpha}$ values. The LEV/TEV interaction also decreases by increasing $\bar{\alpha}$.

Similarly, the second major $C_{l_{\max }}$ increases at higher amplitudes of pitching oscillations. However, the percentage change of the second $C_{l_{\max }}$ is less than that of the first one. That 


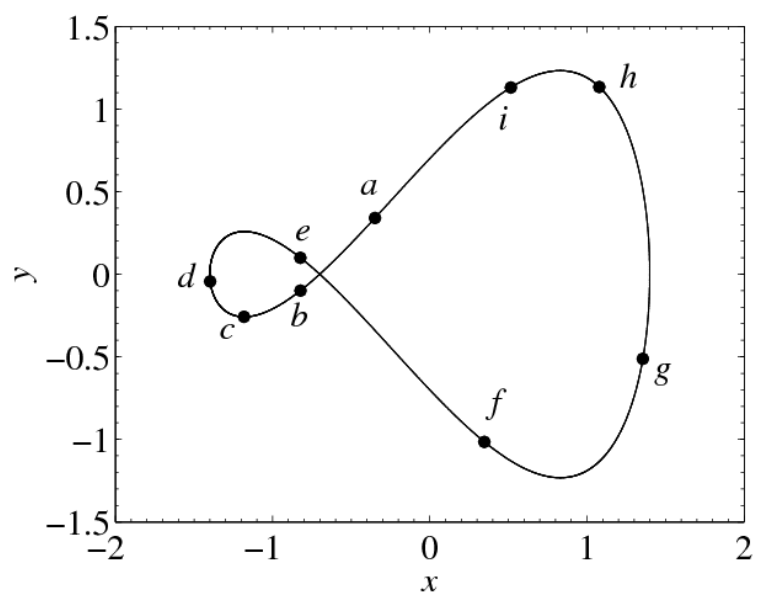

Figure 6.10: Locations of the airfoil on the investigated flapping pattern corresponding to Fig 6.9.

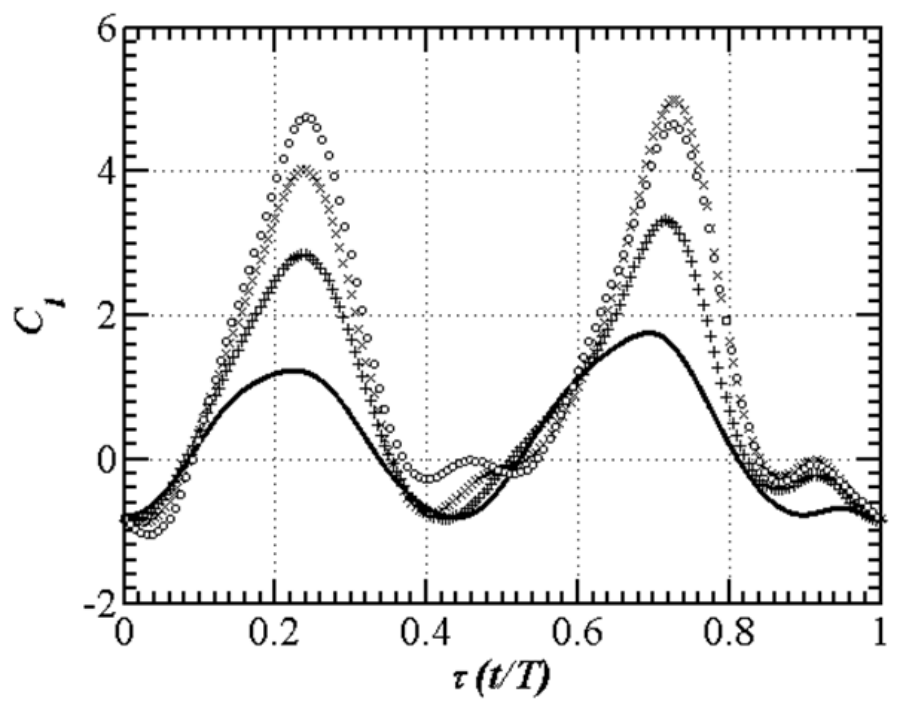

Figure 6.11: Instantaneous lift coefficient $\left(C_{l}\right)$ versus nondimensional time $(\tau)$ for $R=1.4 c$, $d=0.7 c, f=0.25, \alpha_{0}=90^{\circ}, \phi=0, \operatorname{Re}=75:(-), \bar{\alpha}=15^{\circ} ;(+), \bar{\alpha}=30^{\circ} ;(\times), \bar{\alpha}=45^{\circ} ;$ (o), $\bar{\alpha}=60^{\circ}$.

is, $C_{l_{\max }}$ changes for almost $90 \%$ between $\bar{\alpha}=15^{\circ}$ and $30^{\circ}, 43 \%$ between $\bar{\alpha}=30^{\circ}$ and $45^{\circ}$, and $6 \%$ between $\bar{\alpha}=45^{\circ}$ and $60^{\circ}$. It should be mentioned that the second $C_{l_{\max }}$ decreases between $\bar{\alpha}=45^{\circ}$ and $60^{\circ}$, which is against the increasing trend seen between the lower $\bar{\alpha}$ values. This behaviour is accompanied with the generation of a minor peak of $C_{l_{\max }}$ at $\tau$ $\approx 0.46$ and $\bar{\alpha}=60^{\circ}$. Figures 6.13(a) through 6.13(d) show the vortical patterns around 


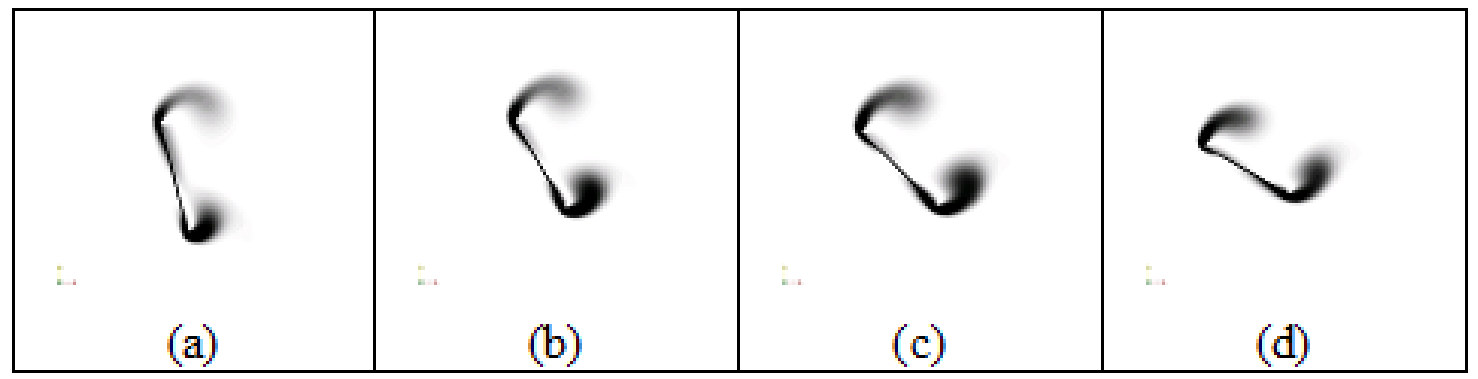

Figure 6.12: LEV and TEV around the ellipse when the first major maximum lift coefficient $\left(C_{l_{\text {max }}}\right)$ is obtained: (a) $\bar{\alpha}=15^{\circ}$; (b) $\bar{\alpha}=30^{\circ}$; (c) $\bar{\alpha}=45^{\circ}$; (d) $\bar{\alpha}=60^{\circ}$.

the airfoil when the second major $C_{l_{\max }}$ is obtained. As observed, when increases, LEVs turn out to be closer to the airfoil surface and the convection of LEV into the downstream wake decreases. TEVs also show a similar behaviour, meaning that their size decreases and they are less convected into the downstream wake at the higher $\bar{\alpha}$. Moreover, the LEV is strengthened when $\bar{\alpha}$ changes from $15^{\circ}$ to $45^{\circ}$, but is weakened from $\bar{\alpha}=45^{\circ}$ to $60^{\circ}$. The weakened LEV at $\bar{\alpha}=60^{\circ}$ could be the main cause for smaller second $C_{l_{\max }}$ than that of $\bar{\alpha}$ $=45^{\circ}$.

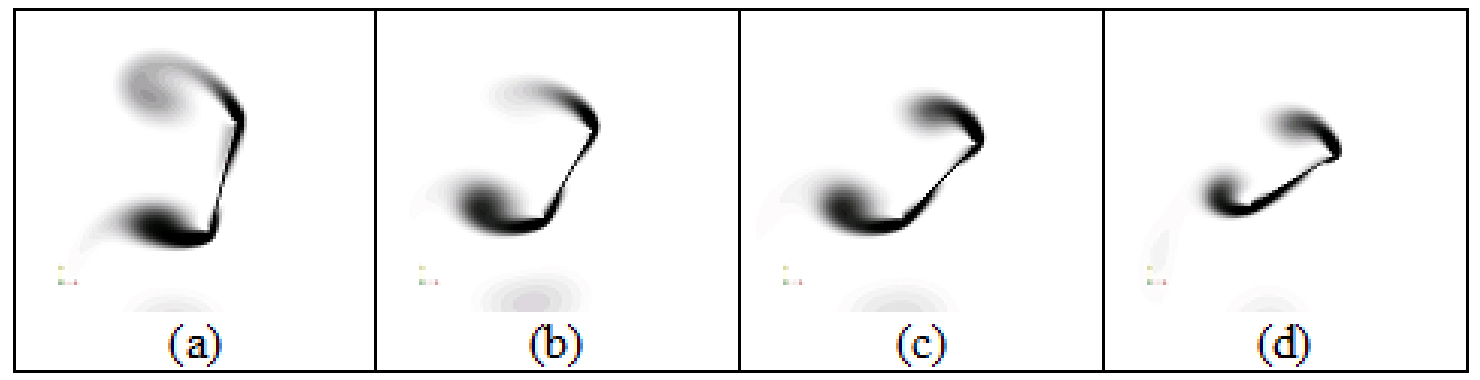

Figure 6.13: LEV and TEV around the ellipse when the second major maximum lift coefficient $\left(C_{l_{\max }}\right)$ is obtained: (a) $\bar{\alpha}=15^{\circ}$; (b) $\bar{\alpha}=30^{\circ}$; (c) $\bar{\alpha}=45^{\circ}$; (d) $\bar{\alpha}=60^{\circ}$.

Figure 6.14 shows the effect of $\bar{\alpha}$ on the drag coefficient $\left(C_{d}\right)$. The amplitude of pitching oscillations has a great influence on the first and second $C_{d_{\max }}$, however the observed effect is less than those on the first and second $C_{l_{\max }}$. As shown, when $\bar{\alpha}$ increases from $15^{\circ}$ to $30^{\circ}$, the first $C_{d_{\max }}$ increases about $8 \%$, but this trend is inversed at the higher $\bar{\alpha}$ values. Moreover, the percentage change of the $C_{d_{\max }}$ values is increased as $\bar{\alpha}$ increases. The absolute value of the second major $C_{d_{\max }}$ is decreased when $\bar{\alpha}$ increases. This effect is accompanied 
by an induced lag in the results. It means that the second $C_{d_{\max }}$ is obtained at $\tau=0.68$, $0.71,0.72$ and 0.73 for $\bar{\alpha}=15^{\circ}, 30^{\circ}, 45^{\circ}$ and $60^{\circ}$, respectively. Higher $\bar{\alpha}$ also generates a minor peak in the $C_{d}$ results which is absent for $\bar{\alpha}=15^{\circ}$, but presents at $\bar{\alpha}=30^{\circ}, 45^{\circ}$ and $60^{\circ}$ at $\tau=0.54,0.5$ and 0.48 , respectively.

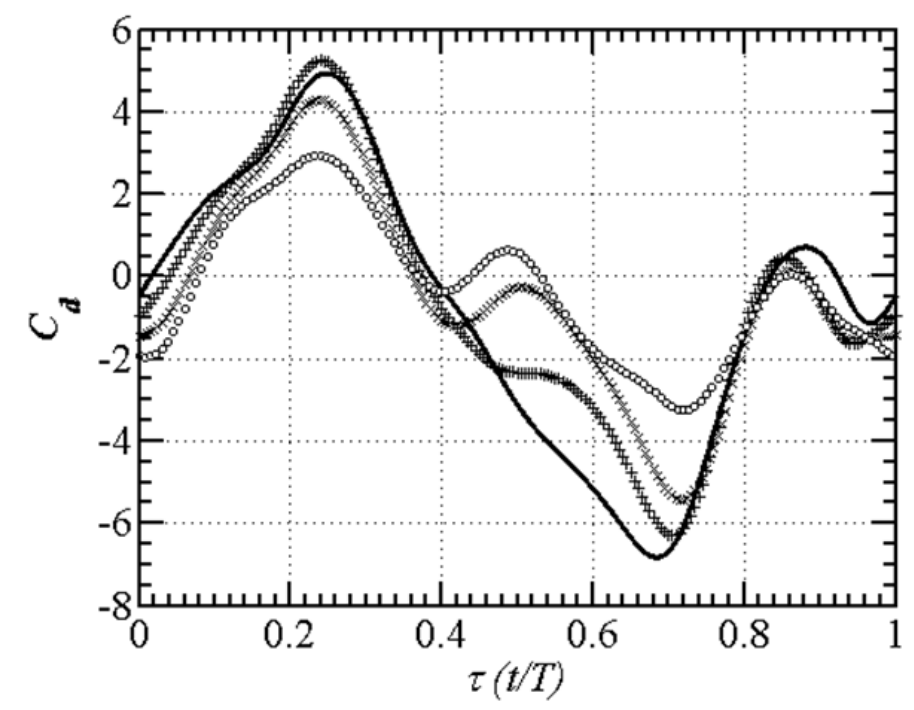

Figure 6.14: Instantaneous lift coefficient $\left(C_{l}\right)$ versus nondimensional time $(\tau)$ for $R=1.4 c$, $d=0.7 c, f=0.25, \alpha_{0}=90^{\circ}, \phi=0, \operatorname{Re}=75:(-), \bar{\alpha}=15^{\circ} ;(+), \bar{\alpha}=30^{\circ} ;(\times), \bar{\alpha}=45^{\circ} ;$ (o), $\bar{\alpha}=60^{\circ}$.

Figure 6.15 also shows the variation of the pitching moment coefficient $\left(C_{m}\right)$ with respect to the $\bar{\alpha}$ variations. The $C_{m}$ major peaks variations are qualitatively similar to those observed in Fig. 6.11.

The mean lift and drag coefficients for the investigated amplitudes of pitching oscillations are calculated and presented in Table 6.1. The mean drag coefficient $\left(\bar{C}_{d}\right)$ is the average between the first and second half stroke mean drag coefficients.

\subsubsection{Effect of the phase angle}

The influence of the phase angle between pitching and plunging motions $(\phi)$ on the fluid flow around the airfoil is investigated for $\phi=-30^{\circ}$ (delayed), $\phi=0$ (symmetrical) and $\phi=$ 


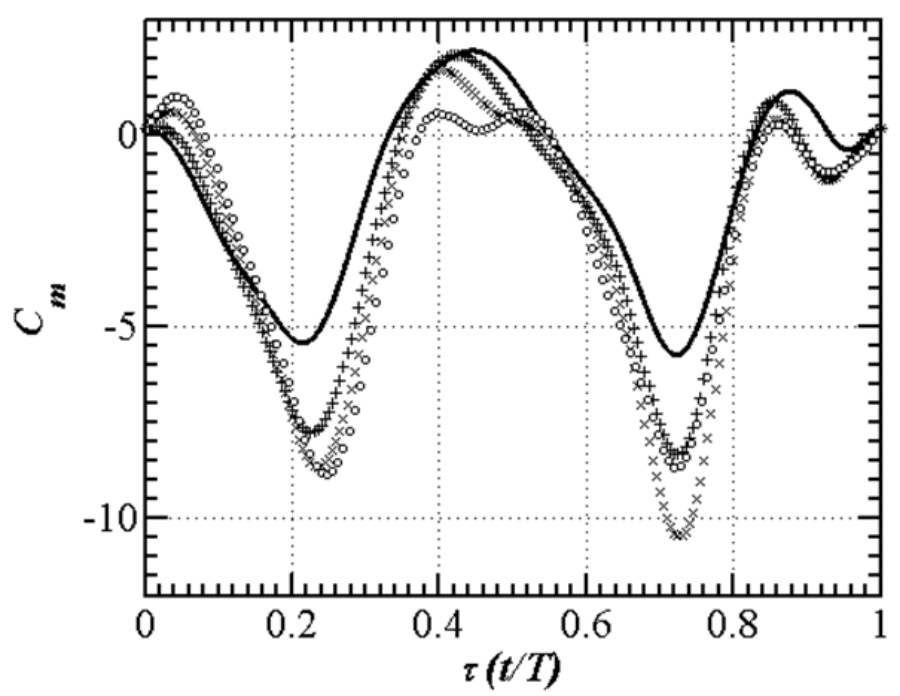

Figure 6.15: Instantaneous pitching moment coefficient $\left(C_{m}\right)$ versus nondimensional time $(\tau)$ for $R=1.4 c, d=0.7 c, f=0.25, \alpha_{0}=90^{\circ}, \phi=0, \operatorname{Re}=75:(-), \bar{\alpha}=15^{\circ} ;(+), \bar{\alpha}=30^{\circ}$; $(\times), \bar{\alpha}=45^{\circ} ;(\circ), \bar{\alpha}=60^{\circ}$.

Table 6.1: Mean lift and drag coefficients $\left(\bar{C}_{l}\right.$ and $\left.\bar{C}_{d}\right)$ for the considered amplitudes of pitching oscillations $(\bar{\alpha})$.

\begin{tabular}{cccccc}
$\bar{\alpha}$ & $\bar{C}_{l}$ & $\bar{C}_{d_{\text {firsthalf }}}$ & $\bar{C}_{d_{\text {secondhalf }}}$ & $\bar{C}_{d}$ & $\bar{C}_{l} / \bar{C}_{d}$ \\
\hline $15^{\circ}$ & 0.253 & 1.61 & -2.999 & 2.305 & 0.11 \\
$30^{\circ}$ & 0.744 & 1.491 & -2.586 & 2.038 & 0.365 \\
$45^{\circ}$ & 1.132 & 1.125 & -2.076 & 1.601 & 0.71 \\
$60^{\circ}$ & 1.258 & 0.857 & -1.444 & 1.15 & 1.1 \\
\hline
\end{tabular}

$30^{\circ}$ (advanced). Based on the following discussions, it is found that the best aerodynamic performance $\left(\frac{\bar{C}_{l}}{\bar{C}_{d}}\right)$ is achieved in symmetrical rotations $(\phi=0)$. The calculated performance is $0.237,0.71$, and 0.614 for $\phi=-30^{\circ}, 0$, and $30^{\circ}$, respectively. Figure 6.16 presents $C_{l}$ versus $\tau$ for these phase angles. As can be seen, the first $C_{l_{\max }}$ decreases from $C_{l_{\max }} \approx 4.53$ at $\phi=$ $-30^{\circ}$ to $C_{l_{\max }} \approx 4.03$ at $\phi=0$. Similarly, the first $C_{l_{\max }}$ decreases from $C_{l_{\max }} \approx 4.03$ at $\phi=$ 0 to $C_{l_{\max }} \approx 1.89$ at $\phi=30^{\circ}$. Hence, the percentage decrease from symmetrical to advanced cases $(53 \%)$ is much higher than that from delayed to symmetrical rotations $(11 \%)$.

On the other hand, $\phi$ has an inverse effect on the second major $C_{l_{\max }}$. That is, the second $C_{l_{\max }}$ increases from $C_{l_{\max }} \approx 1.29$ at $\phi=-30^{\circ}$ to $C_{l_{\max }} \approx 4.82$ at $\phi=0$ and to $C_{l_{\max }} \approx 8.02$ 


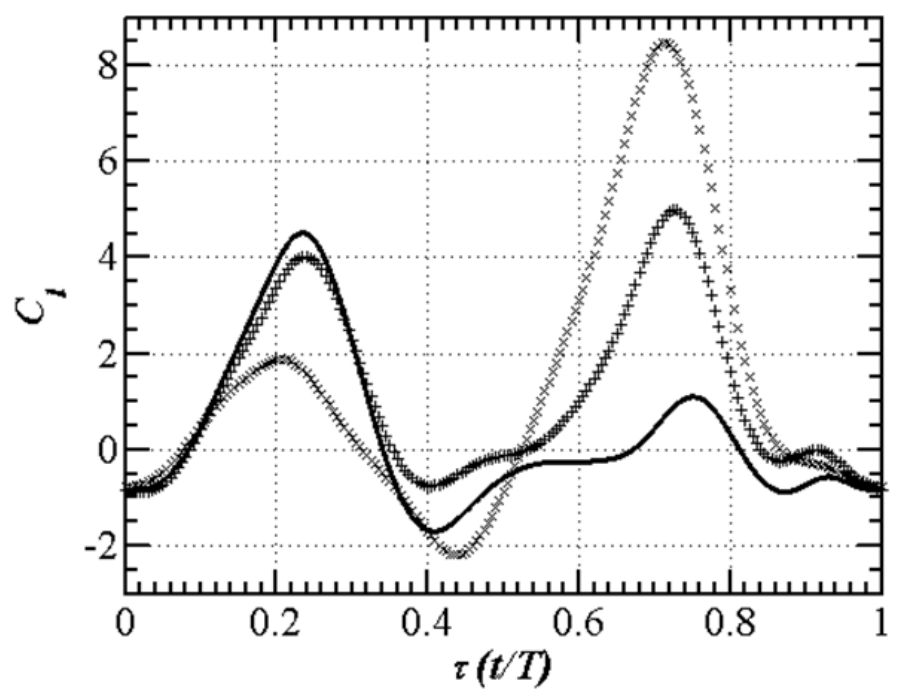

Figure 6.16: Instantaneous lift coefficient $\left(C_{l}\right)$ versus nondimensional time $(\tau)$ for $R=1.4 c$, $d=0.7 c, f=0.25, \alpha_{0}=90^{\circ}, \bar{\alpha}=45^{\circ}$ and $\operatorname{Re}=75:(-), \phi=-30^{\circ} ;(+), \phi=0 ;(\times), \phi=30^{\circ}$.

at $\phi=30^{\circ}$. It means that $\phi$ increases $C_{l_{\max }}$ by $274 \%$ from delayed to symmetric cases and by $66 \%$ from symmetric to advanced rotations. Hence, $\phi$ has a stronger effect on the second major $C_{l_{\max }}$ than on the first one. Moreover, the second major $C_{l_{\max }}$ is obtained at $\tau \approx 0.75$, 0.72 and 0.71 for $\phi=-30^{\circ}, 0$ and $30^{\circ}$, respectively, meaning that higher $\phi$ induces a lead in the second major $C_{l_{\max }}$.

The first and second $C_{l_{\max }}$ corresponding vortical patterns are shown in Figs. 6.17 and 6.18, respectively. As can be seen in Fig. 6.17, when $\phi$ increases from $\phi=-30^{\circ}$ to 0 (Figs. $6.17(\mathrm{a})$ and $6.17(\mathrm{~b}))$ or from $\phi=0$ to $30^{\circ}$ (Figs. 6.17(b) and 6.17(c)) both LEV and TEV are closer to the airfoil surface, less convected into the downstream wake and less interacted with each other. This behavior could be related to the decrease of the first $C_{l_{\max }}$ previously seen in Figure 6.16. However, the LEVs are strengthened by increasing $\phi$, which is the major cause of the increased second major lift coefficient, Fig. 6.18.

Figure 6.19 shows $C_{d}$ versus $\tau$ for $\phi=-30^{\circ}, 0$ and $30^{\circ}$. It can be seen that increasing $\phi$ increases the first major $C_{d_{\max }}$, but it has an inverse effect on the magnitude of the second major peak. Fig. 6.18 also shows the vortical patterns around the airfoil when the second major $C_{l_{\max }}$ is obtained. As can be seen, the airfoil faces its previously separated vortex 


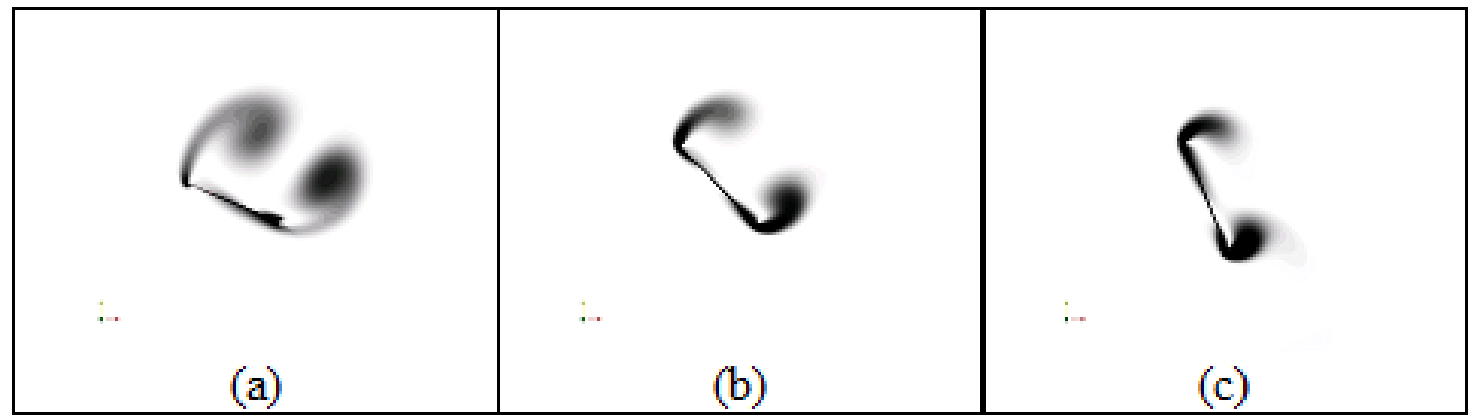

Figure 6.17: Vorticity contours at the first maximum lift coefficient $\left(C_{l_{\max }}\right)$ for $R=1.4 c, d$ $=0.7 c, f=0.25, \alpha_{0}=90^{\circ}, \bar{\alpha}=45^{\circ}$ and $\operatorname{Re}=75$ : (a) $\phi=-30^{\circ}$; (b) $\phi=0$; (c) $\phi=30^{\circ}$.

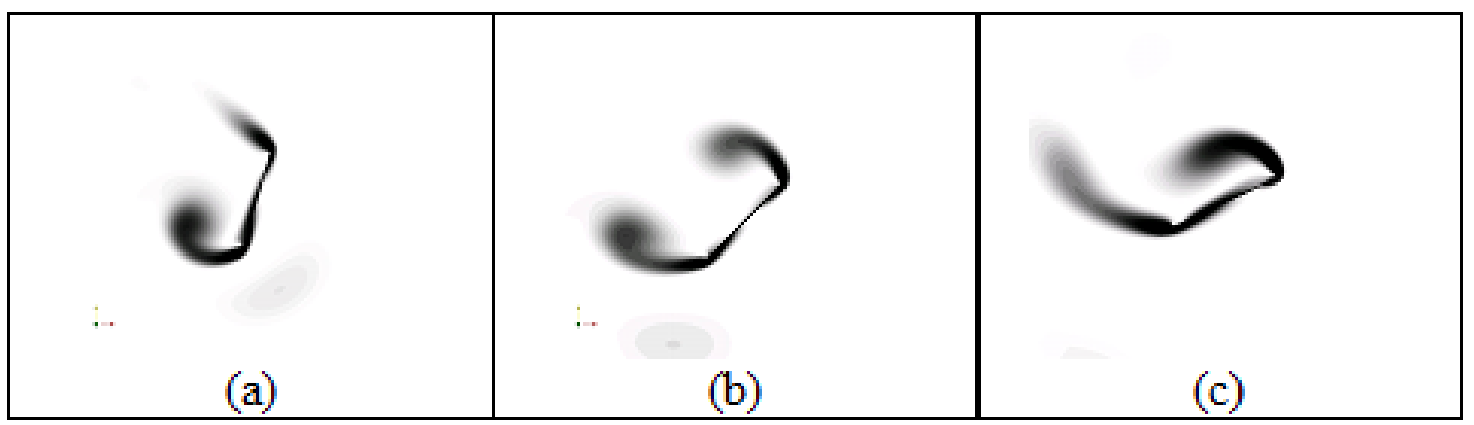

Figure 6.18: Vorticity contours at the second maximum lift coefficient $\left(C_{l_{\max }}\right)$ for $R=1.4 c$, $d=0.7 c, f=0.25, \alpha_{0}=90^{\circ}, \bar{\alpha}=45^{\circ}$ and $\operatorname{Re}=75$ : (a) $\phi=-30^{\circ}$; (b) $\phi=0$; (c) $\phi=30^{\circ}$.

(wake-capturing mechanism) around TE in Fig. 6.18(a). However, Figs. 6.18(b) and 6.18(c) show that this vortex is more deflected away from TE as $\phi$ increases. Moreover, it seems that the TEV strength is just slightly different in Figs. 6.18(a) through 6.18(c), but it is more deflected into the downstream wake. These observations could be the reason for the decreased second $C_{d_{\max }}$. Moreover, increasing $\phi$ from 0 to $30^{\circ}$ induces another peak in $C_{d}$ around $\tau=0.4$ (Fig. 6.19).

Fig. 6.20 shows the variation of the moment coefficient $\left(C_{m}\right)$ versus $\tau$. The first maximum $C_{m}$ is slightly different between $\phi=-30^{\circ}$ and 0 . This could be explained by comparing Figs. 6.17(a) and 6.17(b) where both LEV and TEV are strengthened simultaneously. However, the magnitude of the first maximum $C_{m}$ is decreased at $\phi=30^{\circ}$. It can be seen from Fig. 6.17(c) that TEV is closer to the airfoil surface than that of Fig. 6.17(b), which could result in the decreased magnitude of moment coefficient. Figure 6.20 also shows that the magni- 


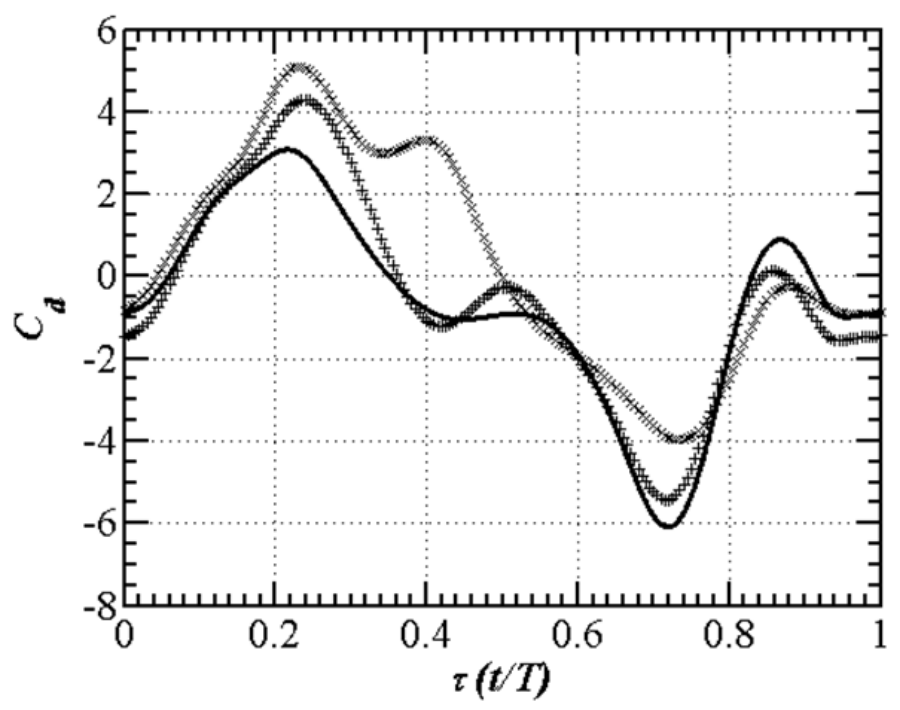

Figure 6.19: Instantaneous drag coefficient $\left(C_{d}\right)$ versus nondimensional time $(\tau)$ for $R=$ $1.4 c, d=0.7 c, f=0.25, \alpha_{0}=90^{\circ}, \bar{\alpha}=45^{\circ}$ and $\operatorname{Re}=75:(-), \phi=-30^{\circ} ;(+), \phi=0 ;(\times), \phi$ $=30^{\circ}$.

tude of the second major moment coefficient is increased at higher $\phi$ values. This could be explained by Figs. 6.18(a)-6.18(c). The LEVs are strengthened significantly and are closer to the airfoil surface as $\phi$ increases; however, the strength of TEVs does not increase as that of LEVs, and TEVs are more deflected into the downstream wake when $\phi$ increases.

The mean lift and drag coefficients as well as the aerodynamic performance are presented in Table 6.2. As can be seen, the highest performance is obtained for the symmetric $(\phi=$ 0) flapping motion.

Table 6.2: Mean lift and drag coefficients $\left(\bar{C}_{l}\right.$ and $\left.\bar{C}_{d}\right)$ for the considered phase angles $(\phi)$.

\begin{tabular}{cccccc}
$\phi$ & $\bar{C}_{l}$ & $\bar{C}_{d_{\text {firsthalf }}}$ & $\bar{C}_{d_{\text {secondhalf }}}$ & $\bar{C}_{d}$ & $\frac{\bar{C}_{l}}{\bar{C}_{d}}$ \\
\hline$-30^{\circ}$ & 0.331 & 0.755 & -2.035 & 1.395 & 0.237 \\
0 & 1.132 & 1.125 & -2.076 & 1.601 & 0.71 \\
$30^{\circ}$ & 1.347 & 2.571 & -1.818 & 2.195 & 0.614 \\
\hline
\end{tabular}




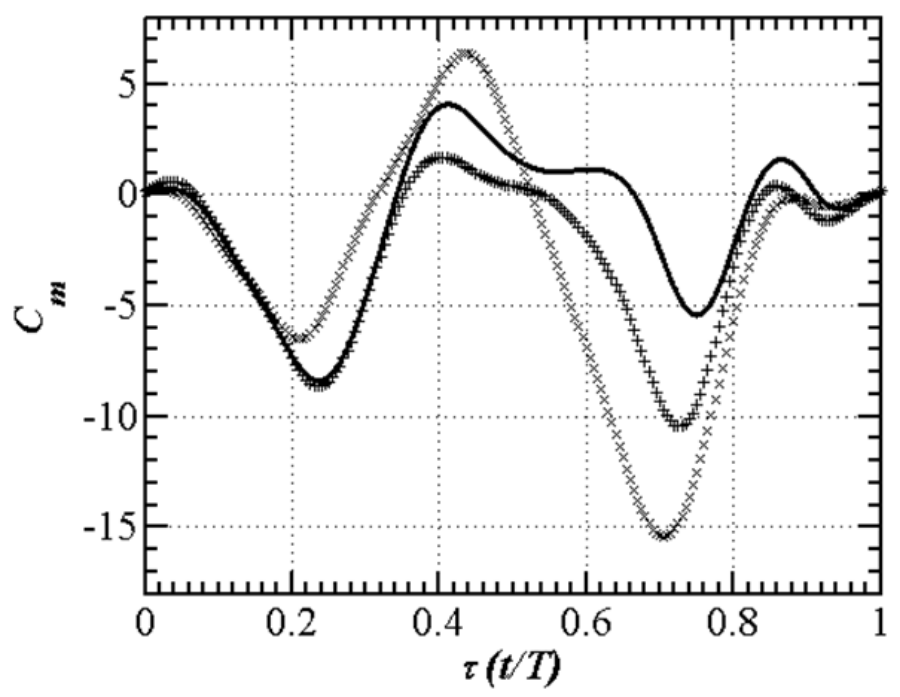

Figure 6.20: Instantaneous pitching moment coefficient $\left(C_{m}\right)$ versus nondimensional time $(\tau)$ for $R=1.4 c, d=0.7 c, f=0.25, \alpha_{0}=90^{\circ}, \bar{\alpha}=45^{\circ}$ and $\operatorname{Re}=75:(-), \phi=-30^{\circ} ;(+), \phi$ $=0 ;(\times), \phi=30^{\circ}$.

\subsubsection{Effect of the mean angle of attack}

The effect of the mean angle of attack $\left(\alpha_{0}\right)$ on the lift coefficient $\left(C_{l}\right)$ is investigated for $\alpha_{0}$ $=45^{\circ}, 60^{\circ}$ and $90^{\circ}$ and presented in Fig. 6.21. As shown, the instantaneous $C_{l}$ at $\alpha_{0}=$ $45^{\circ}$ has two peaks at $\tau \approx 0.1$ and 0.25 . Changing $\alpha_{0}$ from $45^{\circ}$ to $60^{\circ}$ causes the first peak to disappear and increases the value of the second $C_{l_{\max }}$ by $110 \%$ (from $C_{l_{\max }}=0.57$ to 1.3). Furthermore, increasing $\alpha_{0}$ from $60^{\circ}$ to $90^{\circ}$, increases the first $C_{l_{\max }}$ by $212 \%$ (from $C_{l_{\max }}=1.3$ to 4.03 ). Hence, $\alpha_{0}$ has more influence at its higher values (for the investigated kinematics). Conversely, the mean angle of attack has an inverse effect on the second $C_{l_{\max }}$. That is, $C_{l_{\max }}$ decreases from 8.32 to 6.46 when $\alpha_{0}$ increases from $45^{\circ}$ to $60^{\circ}$. This effect is associated with a lag, i.e. the second $C_{l_{\max }}$ for $\alpha_{0}=45^{\circ}, 60^{\circ}$ and $90^{\circ}$ are obtained at $\tau \approx$ 0.66, 0.71 and 0.72 , respectively. Mean angle of attack $\left(\alpha_{0}\right)$ also has a great influence on the second valley of $C_{l}$ at the end of the flapping stroke.

Figures 6.22(a) through 6.22(c) show the vortical patterns around the airfoil when the first major $C_{l_{\max }}$ is obtained. As can be seen, the size of the LEV core is increased at higher $\alpha_{0}$. This change is a measure of the increased LEV strength. Moreover, by increasing $\alpha_{0}$ 


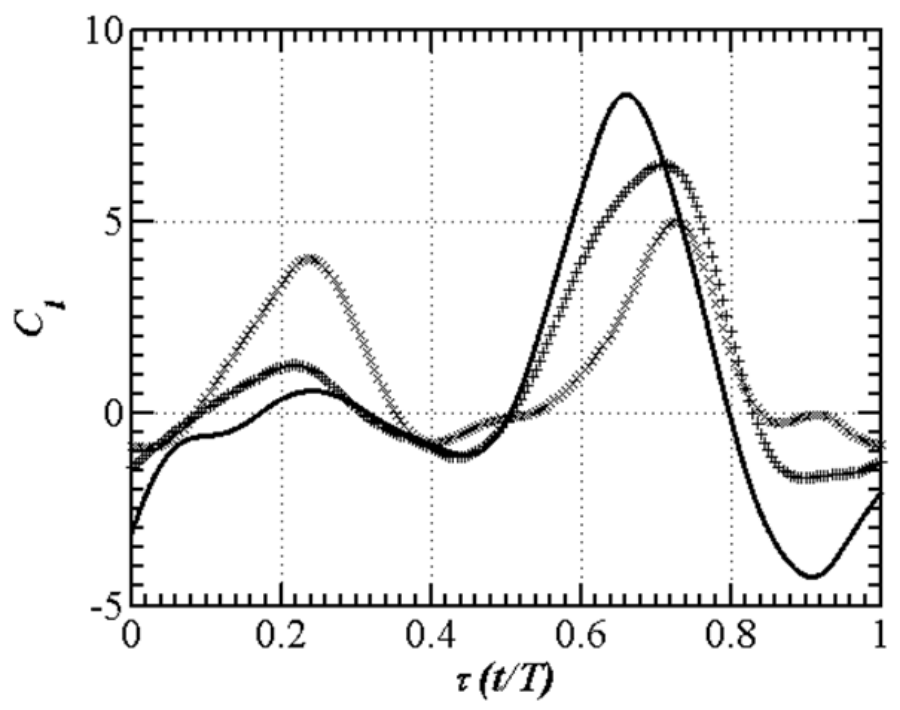

Figure 6.21: Instantaneous lift coefficient $\left(C_{l}\right)$ versus nondimensional time $(\tau)$ for $R=1.4 c$, $d=0.7 c, f=0.25, \bar{\alpha}=45^{\circ}, \phi=0$ and $\operatorname{Re}=75:(-), \alpha_{0}=45^{\circ} ;(+), \alpha_{0}=60^{\circ} ;(\times), \alpha_{0}=$ $90^{\circ}$.

the LEV is deflected towards the airfoil surface. The strength of TEVs, however, does not change significantly, but TEVs are also closer to the airfoil surface. Figs. 6.23(a)-6.23(c) show the vortices around the airfoil when the second major $C_{l_{\max }}$ is obtained. As observed, the LEVs are weakened for higher $\alpha_{0}$, which is the main reason for the decreased second $C_{l_{\max }}$.

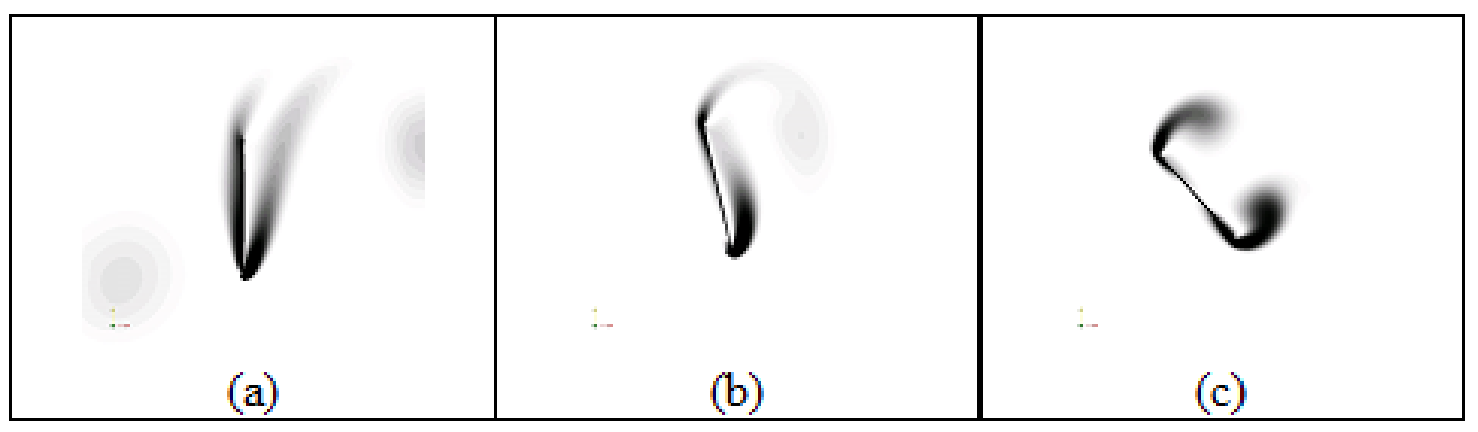

Figure 6.22: Vorticity contours at the first maximum lift coefficient $\left(C_{l_{\max }}\right)$ for $R=1.4 c, d$ $=0.7 c, f=0.25, \bar{\alpha}=45^{\circ}, \phi=0$ and $\operatorname{Re}=75$ : (a) $\alpha_{0}=45^{\circ}$; (b) $\alpha_{0}=60^{\circ}$; (c) $\alpha_{0}=90^{\circ}$. 


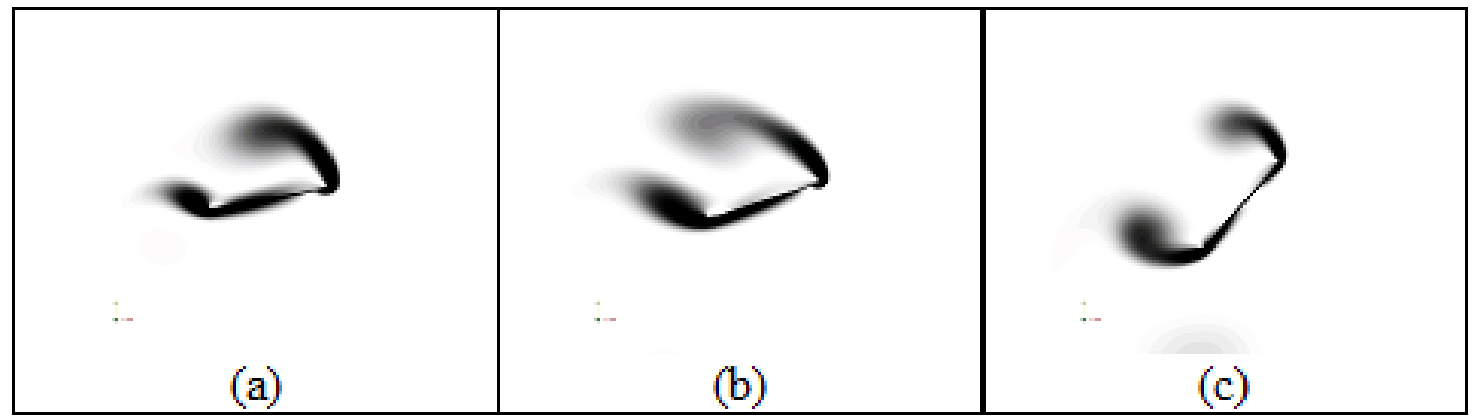

Figure 6.23: Vorticity contours at the second maximum lift coefficient $\left(C_{l_{\max }}\right)$ for $R=1.4 c$, $d=0.7 c, f=0.25, \bar{\alpha}=45^{\circ}, \phi=0$ and $\operatorname{Re}=75$ : (a) $\alpha_{0}=45^{\circ}$; (b) $\alpha_{0}=60^{\circ}$; (c) $\alpha_{0}=90^{\circ}$.

\subsubsection{Effect of the Reynolds number}

Effect of the Reynolds number (Re) on the airfoil's fluid dynamic characteristics is investigated for $\operatorname{Re}=37.5,75$ and 150. It is observed that Re does not change the instantaneous force and moment coefficients significantly, and increasing Re slightly increases the aerodynamic performance. Figure 6.24 shows $C_{l}$ versus $\tau$ for the investigated Re range. As shown, Re does not have any noticeable effect on the first $C_{l_{\max }}$. It changes the first $C_{l_{\max }}$ from 3.72 at $\operatorname{Re}=37.5$ to 4.03 at $\operatorname{Re}=75$. The effect is even less when Re changes from 75 to 150 , where the first $C_{l_{\max }}$ remains almost unchanged. Similarly, the second $C_{l_{\max }}$ increases from 4.03 at $\operatorname{Re}=37.5$ to 4.98 at $\operatorname{Re}=75$, and remains unchanged between $\operatorname{Re}=75$ and 150 . However, Re has more influence on the second $C_{l_{m a x}}$. Despite the negligible influence of Re on the major $C_{l_{\max }}$, the minor peak at the end of the flapping period changes considerably with Re.

Figures $6.25(\mathrm{a})$ and $6.25(\mathrm{~b})$ show first major $C_{l_{\max }}$ corresponding vortical patterns for $\operatorname{Re}=37.5$ and 75 , respectively. The vortices at $\operatorname{Re}=150$ are not shown as they are very similar to those at $\mathrm{Re}=75$. It can be seen that the size of both LEV and TEV cores is increased by increasing Re. This trend is also observed in Figs. 6.25(c) and 6.25(d), showing the respective vortices at second major $C_{l_{\max }}$. Therefore, increasing Re from $\operatorname{Re}=37.5$ to 75 strengthens both LEV and TEV, leading to the increased first and second $C_{l_{\max }}$.

The airfoil's pitching moment coefficients at the investigated Re range are shown in Fig. 


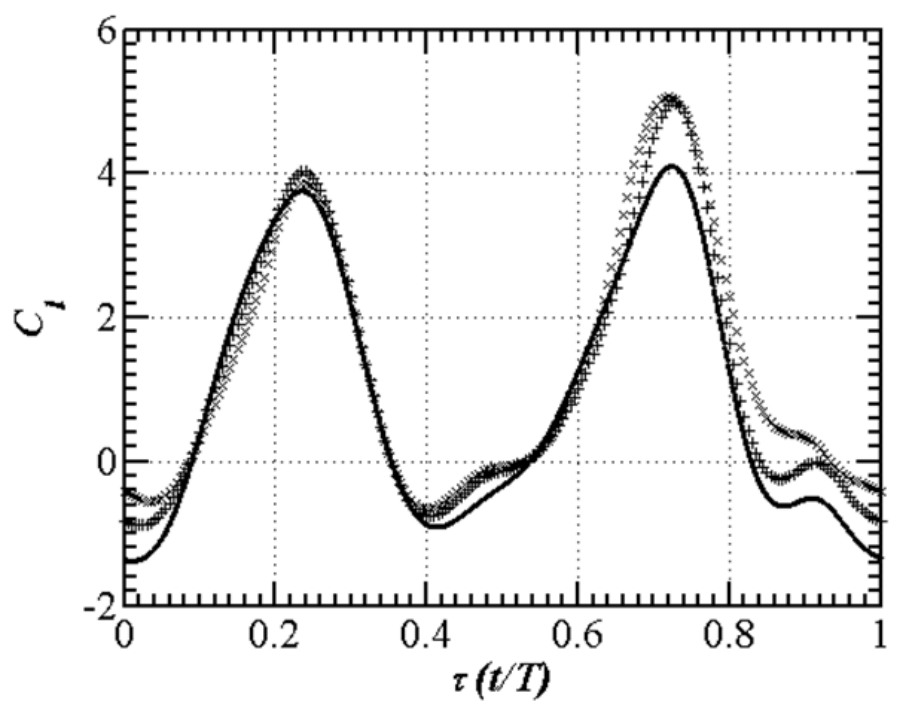

Figure 6.24: Instantaneous lift coefficient $\left(C_{l}\right)$ versus nondimensional time $(\tau)$ for $R=1.4 c$, $d=0.7 c, f=0.25, \alpha_{0}=90^{\circ}, \bar{\alpha}=45^{\circ}, \phi=0:(-), \operatorname{Re}=37.5 ;(+), \operatorname{Re}=75 ;(\times), \operatorname{Re}=150$.

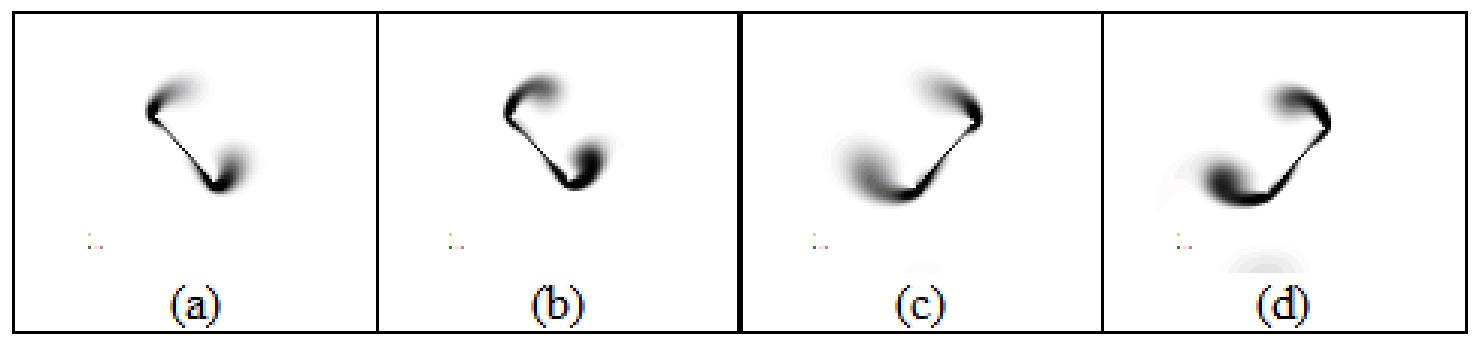

Figure 6.25: Vorticity contours for $R=1.4 c, d=0.7 c, f=0.25, \alpha_{0}=90^{\circ}, \bar{\alpha}=45^{\circ}, \phi=0$ : (a) first $C_{l_{\max }}$ at $\operatorname{Re}=37.5$; (b) first $C_{l_{\max }}$ at $\operatorname{Re}=75$; (c) second $C_{l_{\max }}$ at $\operatorname{Re}=37.5$; (d) second $C_{l_{\max }}$ at $\operatorname{Re}=75$.

6.26. As shown, the effect of $\operatorname{Re}$ on $C_{m}$ peaks is more than that on the $C_{l}$ peaks.

The mean lift and drag coefficients as well as the aerodynamic performance for the considered Reynolds numbers are presented in Table 6.3. As can be seen, higher Re increases the aerodynamic performance.

\subsubsection{Effect of Strouhal Number}

The effect of the Strouhal number $(\mathrm{St})$ on $C_{l}$ and $C_{d}$ is investigated by changing the vertical and horizontal amplitudes of translational oscillations $(R$ and $d)$. Based on the following 


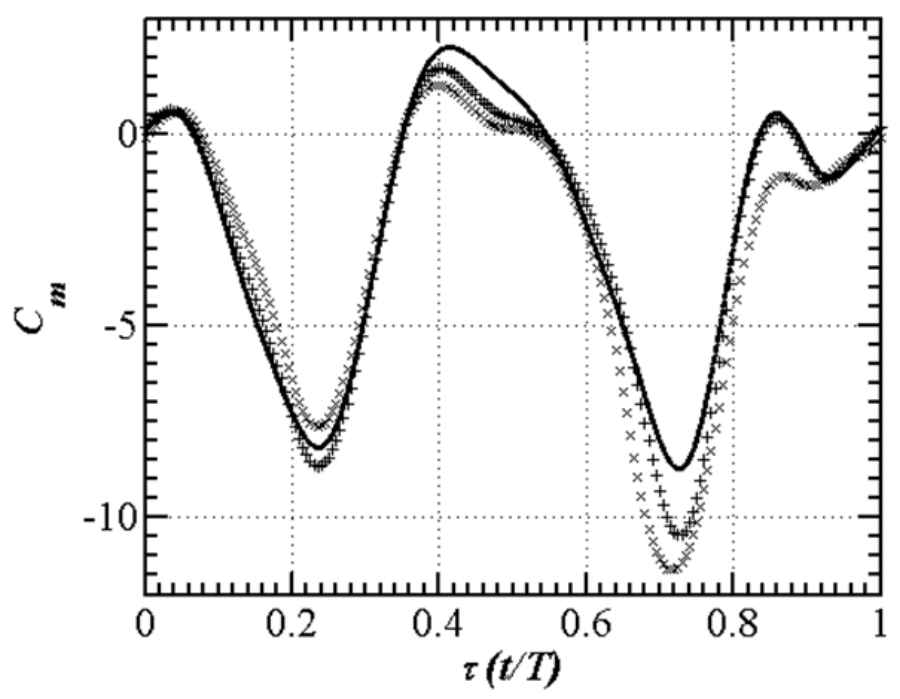

Figure 6.26: Instantaneous pitching moment coefficient $\left(C_{m}\right)$ versus nondimensional time $(\tau)$ for $R=1.4 c, d=0.7 c, f=0.25, \alpha_{0}=90^{\circ}, \bar{\alpha}=45^{\circ}, \phi=0:(-), \operatorname{Re}=37.5 ;(+), \operatorname{Re}=$ $75 ;(\times), \operatorname{Re}=150$.

Table 6.3: Mean lift and drag coefficients $\left(\bar{C}_{l}\right.$ and $\left.\bar{C}_{d}\right)$ for the considered Re.

\begin{tabular}{cccccc}
$\operatorname{Re}$ & $\bar{C}_{l}$ & $\bar{C}_{d_{\text {firsthalf }}}$ & $\bar{C}_{d_{\text {secondhalf }}}$ & $\bar{C}_{d}$ & $\bar{C}_{l}$ \\
\hline 37.5 & 0.914 & 1.234 & -2.171 & 1.702 & 0.536 \\
75 & 1.132 & 1.125 & -2.076 & 1.601 & 0.71 \\
150 & 1.287 & 1.091 & -2.089 & 1.589 & 0.81 \\
\hline
\end{tabular}

discussions, it is found that St does not have a significant effect on the aerodynamic performance. That is, $\frac{\bar{C}_{l}}{\bar{C}_{d}}$ is $0.664,0.71$ and 0.672 for $\mathrm{St}=0.116,0.113$ and 0.11 . Figure 6.27 shows $C_{l}$ versus $\tau$ for $\mathrm{St}=0.116,0.113$ and 0.110 corresponding to $d=0.4 c, 0.7 c$ and $c$, respectively.

As shown, the first $C_{l_{\max }}$ increases from 3.82 at $\mathrm{St}=0.116$ to 4.03 at $\mathrm{St}=0.113$. This increase is associated with a lag in the $C_{l_{\max }}$. That is, $C_{l_{\max }}$ is achieved at $\tau \approx 0.22$ for $\mathrm{St}=$ 0.116 and at $\tau \approx 0.23$ for $\mathrm{St}=0.113$. Conversely, the peak decreases from 4.03 to 3.7 for $\mathrm{St}=$ 0.113 to 0.110 ; however, the induced lag is still observed, and the first $C_{l_{\max }}$ is obtained at $\tau$ $\approx 0.24$ for $\mathrm{St}=0.110$. The increasing/decreasing trend is also observed for the second $C_{l_{\max }}$, but the lag is converted to a lead, i.e. the second $C_{l_{\max }}$ is obtained at $\tau \approx 0.73,0.72$ and 


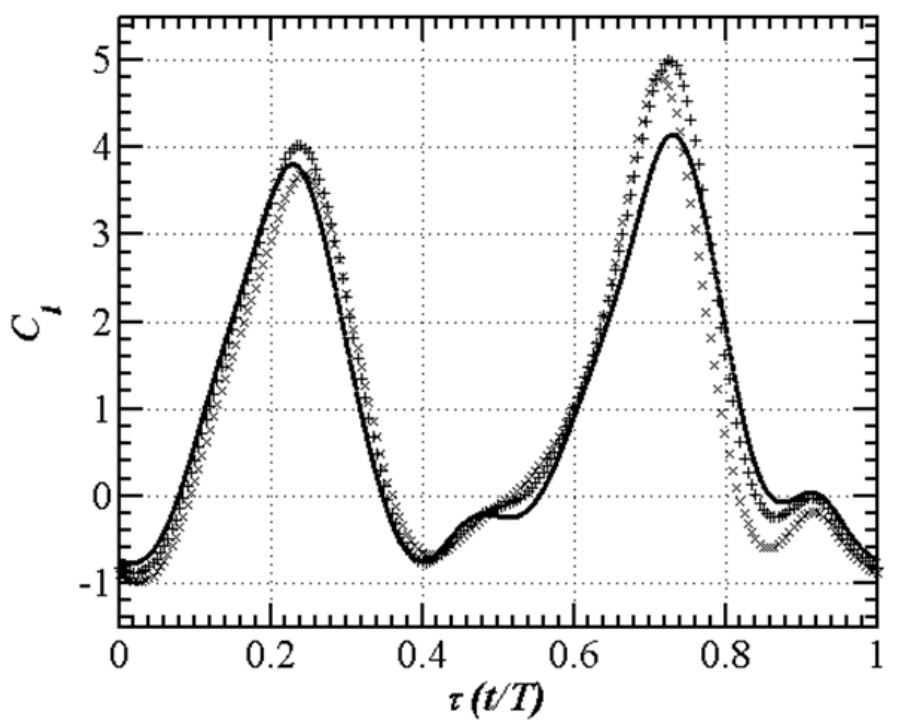

Figure 6.27: Instantaneous lift coefficient $\left(C_{l}\right)$ versus nondimensional time $(\tau)$ for $R=1.4 c$, $f=0.25, \alpha_{0}=90^{\circ}, \bar{\alpha}=45^{\circ}, \phi=0$ and $\operatorname{Re}=75:(-)$, St $=0.116 ;(+)$, St $=0.113 ;(\times)$, St $=0.110$.

0.71 for $\mathrm{St}=0.116,0.113$ and 0.110 , respectively. St also has similar effects on $C_{d}$ (Fig. 6.28).

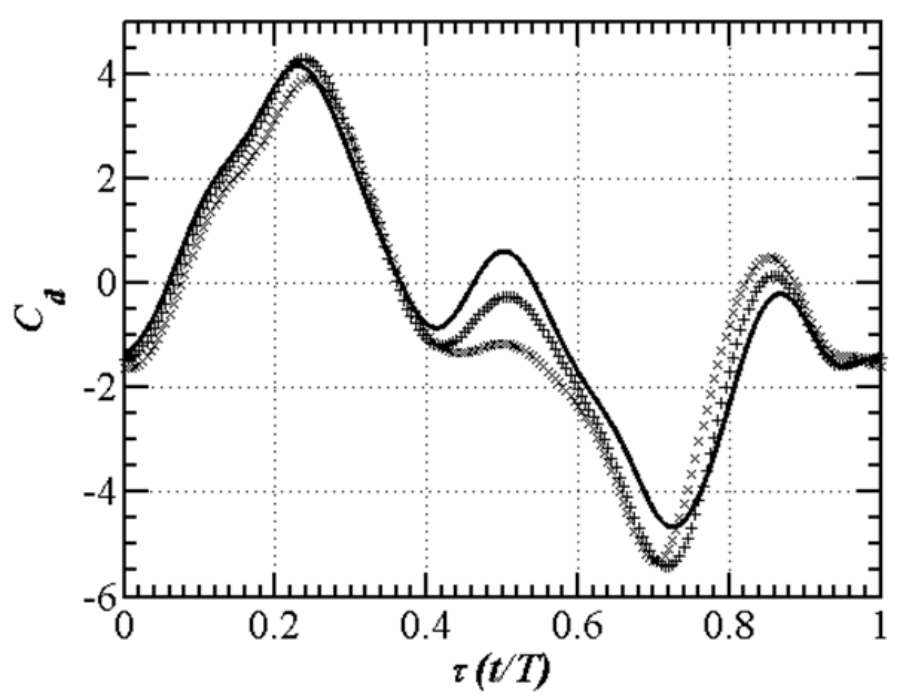

Figure 6.28: Instantaneous drag coefficient $\left(C_{d}\right)$ versus nondimensional time $(\tau)$ for $R=$ $1.4 c, f=0.25, \alpha_{0}=90^{\circ}, \bar{\alpha}=45^{\circ}, \phi=0$ and $\operatorname{Re}=75:(-)$, St $=0.116 ;(+)$, St $=0.113$; $(\times)$, St $=0.110$.

The mean lift and drag coefficients as well as the aerodynamic performance for the 
considered Strouhal numbers (based on $d$ ) are presented in Table 6.4.

Table 6.4: Mean lift and drag coefficients $\left(\bar{C}_{l}\right.$ and $\left.\bar{C}_{d}\right)$ for the considered St.

\begin{tabular}{cccccc} 
St & $\bar{C}_{l}$ & $\bar{C}_{d_{\text {firsthalf }}}$ & $\bar{C}_{d_{\text {secondhalf }}}$ & $\bar{C}_{d}$ & $\frac{\bar{C}_{l}}{\bar{C}_{d}}$ \\
\hline 0.116 & 1.051 & 1.286 & -1.877 & 1.582 & 0.664 \\
0.113 & 1.132 & 1.125 & -2.076 & 1.601 & 0.71 \\
0.11 & 0.982 & 0.888 & -2.038 & 1.463 & 0.672 \\
\hline
\end{tabular}

The effect of St is also investigated by changing $R$ from $c$ to $1.4 c$ corresponding to $\mathrm{St}=$ 0.153 and 0.113 , respectively. As shown in Fig. 6.29, the first $C_{l_{\max }}$ decreases from 6.21 at $\tau \approx 0.22$ to 4.03 at $\tau \approx 0.23$. The second major $C_{l_{\max }}$ also decreases from $C_{l_{\max }}=5.62$ at $\mathrm{St}=0.153$ to $C_{l_{\max }}=4.92$ at $\mathrm{St}=0.113$. This trend is associated with a lead observed in the data.

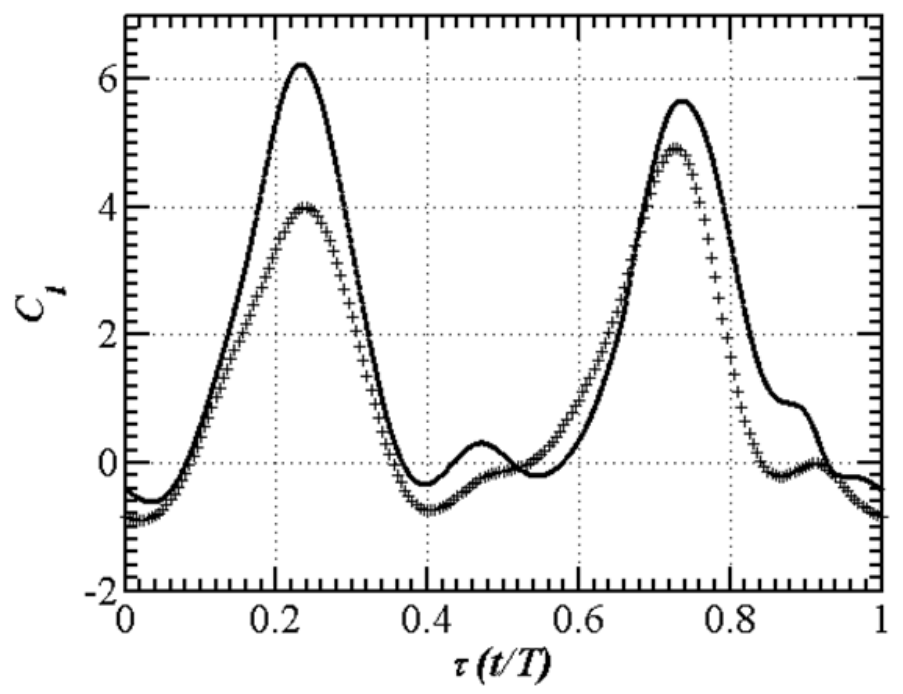

Figure 6.29: Instantaneous lift coefficient $\left(C_{l}\right)$ versus nondimensional time $(\tau)$ for $d=0.7 c$, $f=0.25, \alpha_{0}=90^{\circ}, \bar{\alpha}=45^{\circ}, \phi=0$ and Re $=75:(-)$, St $=0.153 ;(+)$, St $=0.113$.

\subsubsection{Effect of the pitching axis location}

The effect of the pitching axis location on the airfoil's $C_{l}$ and $C_{m}$ is investigated, Figs. 6.30 and 6.31. Two locations $x / c=0.25$ and 0.5 , measured from the airfoil LE, are considered. 
As shown in Figs. 6.30 and 6.31 , the variation of $x / c$ affects the minor peaks of $C_{l}$ and $C_{m}$, but it has negligible effects on the major peaks.

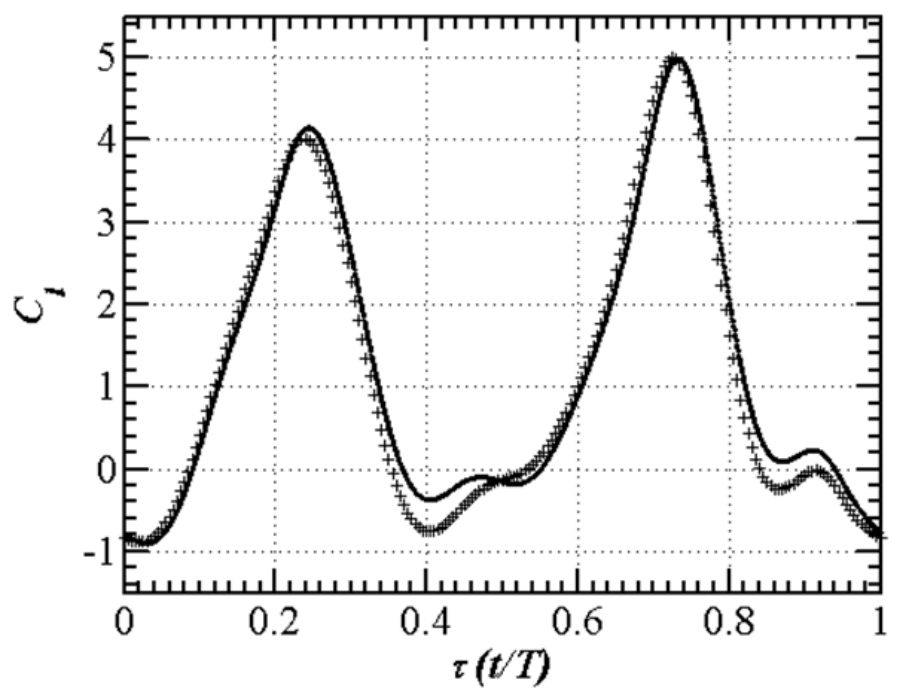

Figure 6.30: Instantaneous lift coefficient $\left(C_{l}\right)$ versus nondimensional time $(\tau)$ for $R=1.4 c$, $d=0.7 c, f=0.25, \alpha_{0}=90^{\circ}, \bar{\alpha}=45^{\circ}, \phi=0$ and $\operatorname{Re}=75:(-), x / c=0.25 ;(+), x / c=0.5$.

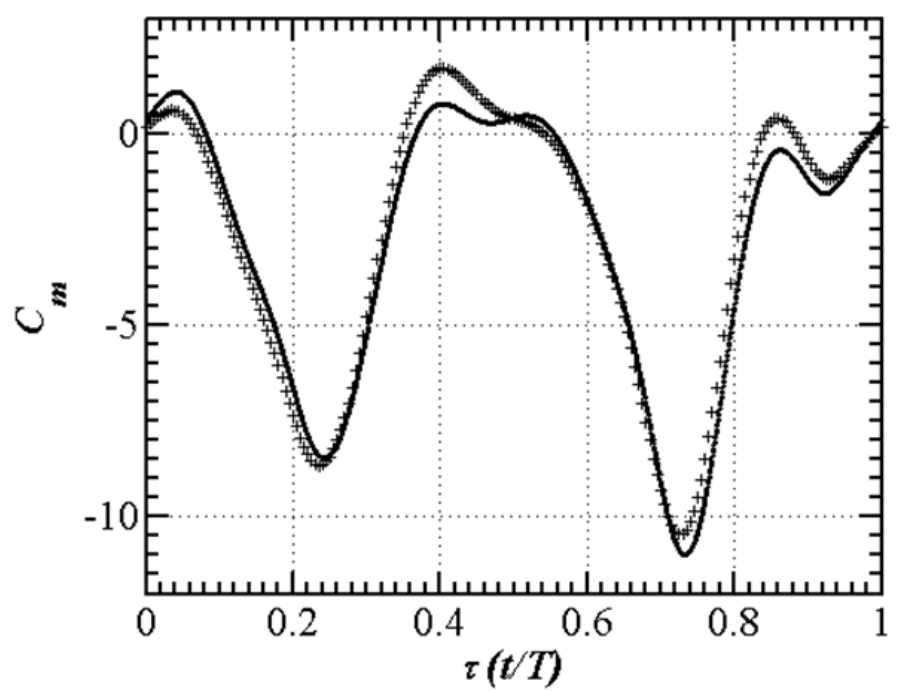

Figure 6.31: Instantaneous pitching moment coefficient $\left(C_{m}\right)$ versus nondimensional time $(\tau)$ for $R=1.4 c, d=0.7 c, f=0.25, \alpha_{0}=90^{\circ}, \bar{\alpha}=45^{\circ}, \phi=0$ and $\operatorname{Re}=75:(-), x / c=$ $0.25 ;(+), x / c=0.5$. 


\subsection{Summary and conclusion}

CFD simulations of a flapping airfoil in LRN flow are conducted using 2-D N-S equations based on the FVM approach. An extensive parametric study is performed and the effects of flow and system parameters on the lift, drag and pitching moment coefficients as well as the vortical patterns around the airfoil are investigated. It is observed that the pitching amplitude of oscillations, phase angle, and mean angle of attack affect the force and moment coefficients noticeably. They change the peak values of the force and moment coefficients. The strength of the vortical patterns around the model is also affected by the flow and system parameters, and it is shown that the LEV/TEV interactions as well as their convection into the downstream wake of the airfoil are also dependent on the investigated parameters. Increasing the amplitude of pitching oscillations increases the aerodynamic performance. Moreover, it is shown that the best aerodynamic performance is obtained in the symmetrical oscillations. Although Re and St are of great importance to the unsteady forces, their influence turn out to be of secondary importance to the fluid dynamics compared to those of the amplitude of pitching oscillations, phase angle and mean angle of attack. Increasing Re slightly increases the aerodynamic performance, but St (based on the amplitude of translational oscillations) does not have any noticeable effect on the performance (at least for the investigated magnitudes). The pitching axis location seems to influence only the minor peaks of the force and moment coefficients. The results from this 2-D study may provide

valuable information about the respective 3-D figure-of-eight like flapping wing [58]. The present study reveals important information about influential flow and system parameters and their effects on the performance characteristics. The present results may be used in the respective $3-\mathrm{D}$ future studies, when the $2-\mathrm{D}$ and $3-\mathrm{D}$ results will be compared and the importance of 3-D effects and vortical interactions will be investigated. In the next chapter, the unsteady aerodynamics of a pair of pitching airfoils is thoroughly investigated. 


\section{Chapter 7}

\section{The unsteady aerodynamics of a pair of pitching airfoils}

The objective of the present chapter is to investigate the LRN fluid dynamics of two airfoils in pitching oscillations. The airfoils are in a tandem configuration and perform in-phase oscillations. Navier-Stokes (NS) equations with Finite Volume Method (FVM) are used and the instantaneous aerodynamic force coefficients are analyzed. The effect of amplitude of pitching oscillations and Re are investigated on the fluid forces. It is found that the amplitude of pitching oscillations is of primary importance to the fluid forces, affecting them both quantitatively and qualitatively. As it will be discussed in details in the following sections, Re is found to be of secondary importance compared to the effects of the amplitude of pitching oscillations. It mainly affects the magnitude of the forces.

\subsection{Introduction}

Pitching oscillations occur frequently in a wide range of applications. These oscillations can be in the form of single oscillating bodies, such as the oscillations of an aircraft wing, or multipitching bodies such as the relative pitching oscillations between compressor blades. The oscillations could also be destructive to the fluid dynamics and structure, such as wing flut- 
ter, or assistive in terms of the force generation. For example, Low-Reynolds-Number (LRN) flapping motions consist of pitching and plunging portions. These unsteady conditions can occur between several existing airfoils/blades, and their interaction can increase/decrease the fluid forces, depending on the governing conditions.

Unfortunately, less attention has been paid to LRN multi-bodies under both pitching and plunging motions. A clear understanding of the fluid dynamics of multi-oscillating bodies, their interaction, and the effects of major parameters on their performance can result in improved designs. Classical theoretical techniques such as Theodorsen's method yield acceptable results only for small displacements and single bodies [8]. CFD simulations stand out as the most common means of solution method in LRN flows. Certain numerical studies have been conducted on multi-bodies oscillations and the effects of a number of unsteady flow and system parameters on their aerodynamic characteristics were investigated [62], [97] and [102].

The objective of the present chapter is to investigate the instantaneous unsteady force coefficients of a pair of airfoils under harmonically pitching oscillations by developing generalized grid interface (GGI) method to multi-body rotations [132]. The airfoils oscillate with the same phase angle in a tandem configuration. Computational Fluid Dynamics (CFD) is used to solve the 2D Navier-Stokes (NS) governing equations discretized based on Finite Volume Method (FVM). The effects of the amplitude of pitching oscillations and Re on the instantaneous unsteady forces are explored.

\subsection{Numerical simulation method}

The flow field of a pair of pitching airfoils (the centre-to-centre spacing is $4 c$ ) is governed by Equations 3.7. The Reynolds number (Re) defined in Equations 3.10 is based on the freestream velocity $\left(U_{\infty}\right)$. The schematic of the airfoils in the computational domain is shown in Fig 7.1. 


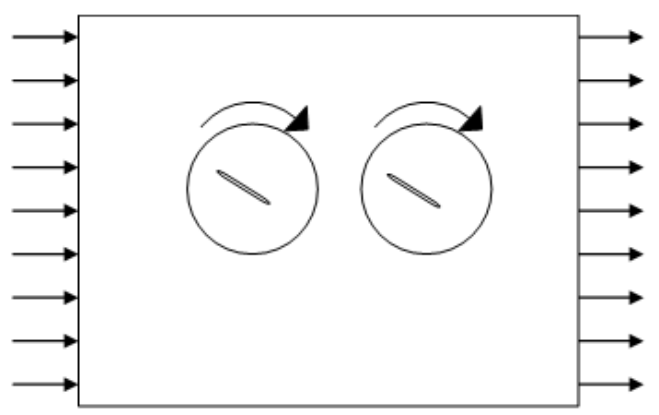

Figure 7.1: Schematic of the computational domain and the pitching airfoils.

The fluid dynamics of two flat plates in tandem configuration is investigated based on FVM and N-S equations. The computations are based on a second order central differencing scheme for convective and diffusive terms and a second order Euler implicit scheme for temporal discretization. The resulting linear system of equations is treated with Preconditioned Conjugate Gradient (PCG) solvers, and the Pressure Implicit and Splitting of Operators (PISO) algorithm is used for the pressure-velocity coupling. The kinematics of the airfoils is governed by:

$$
\alpha(t)=\alpha_{0}+\theta \cos (2 \pi f t+\phi)
$$

where $\alpha(t)$ is the instantaneous angle of attack, $\alpha_{0}$ and $\theta$ are the mean angle of attack and amplitude of oscillations, respectively, and $\phi$ is the phase lead/lag. In the present study, $\alpha_{0}=0, \phi=0$ and $f=2 \mathrm{~Hz}$. The mesh motion is performed using the Generalized Grid Interface (GGI) algorithm [132], which is extended to perform multi-body rotations.

\subsection{Evaluation of the results}

The grid sensitivity analysis is first conducted using three mesh sizes of 23,47 , and $94\left(\times 10^{3}\right)$ cells, having 2000 time steps for the temporal resolution. Figure 7.2 shows that $47 \times 10^{3}$ is reasonably refined to yield grid independent solutions. Different temporal resolutions (500, 1000, 2000, and 4000 time steps in a period) are also examined for a mesh size of $47 \times 10^{3}$ 
cells. The results indicate that the temporal resolution of 2000 is enough to ensure temporally independent solutions. Hence, the simulations are conducted for $47 \times 10^{3}$ cells and 2000 time steps.

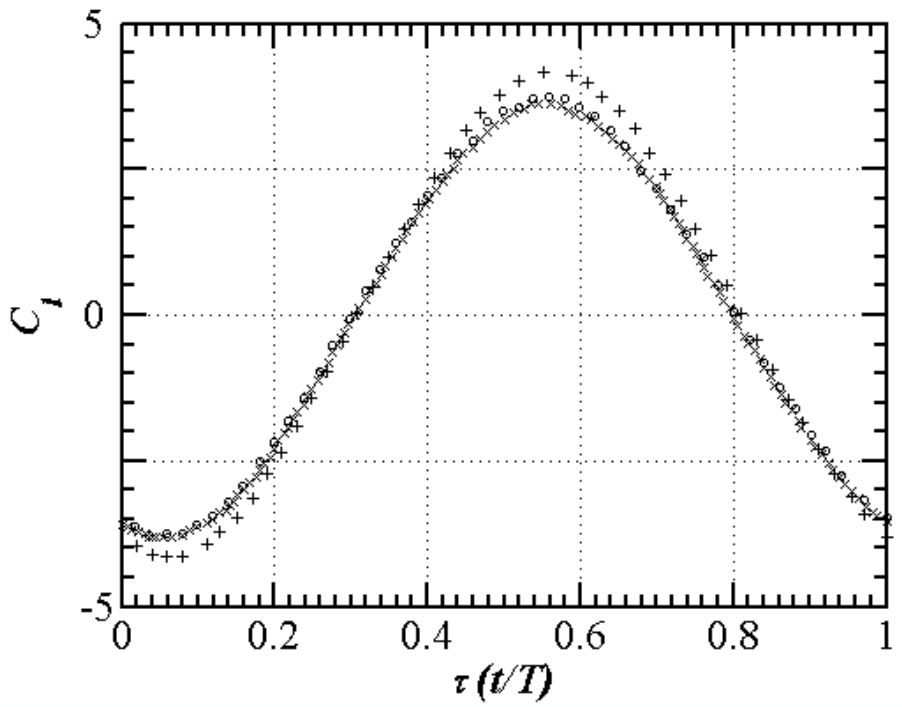

Figure 7.2: Instantaneous lift coefficient $\left(C_{l}\right)$ versus nondimensional time $(\tau)$ for $\operatorname{Re}=50$ and $\theta=15^{\circ}:(+), 23 \times 10^{3} ;(\mathrm{o}), 47 \times 10^{3},(\times), 94 \times 10^{3}$.

Due to the lack of published studies on multi-pitching airfoils, the pitching oscillations of a single NACA0012 airfoil is first simulated. As mentioned above, the mesh motion is based on GGI algorithm, which is extended to multi-body rotations. Therefore, the accuracy of the simulations using GGI is investigated following the study reported in [68]. Akbari and Price [68] studied the pitching oscillation of a single NACA0012 airfoil at Re $=3000$, governed by the following equation:

$$
\alpha(t)=\alpha_{0}+A \cos (2 \pi f t)
$$

with $\alpha_{0}=15^{\circ}, \mathrm{A}=10^{\circ}$ and $f=0.08$. The simulated $C_{d}$ (Fig. 7.3) is within good agreement with that of Akbari and Price [68]. The difference could be attributed to the different LE/TE shapes of the airfoils considered in the two studies. 


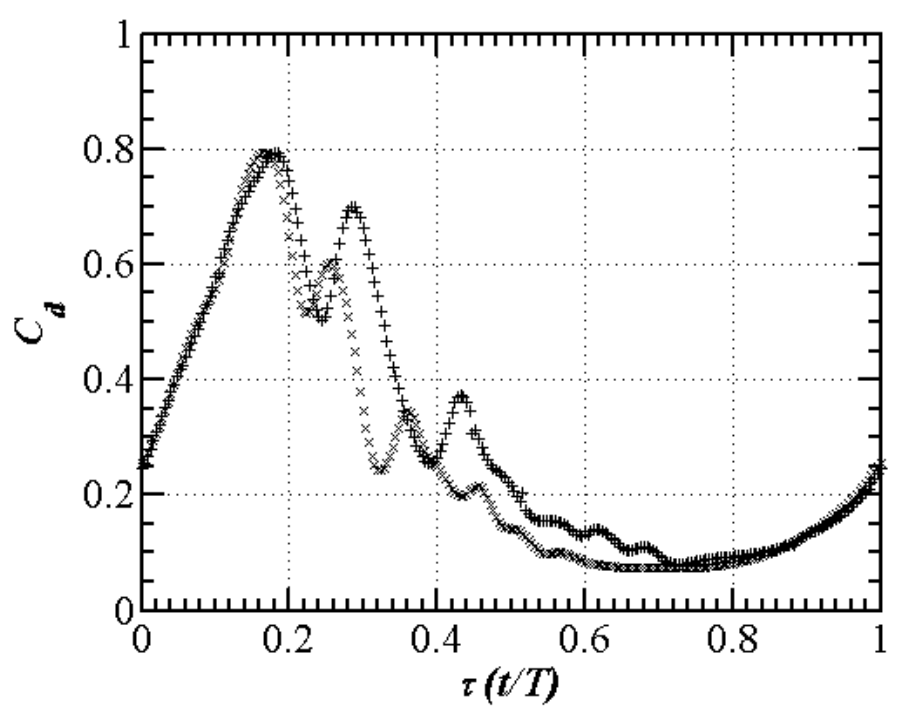

Figure 7.3: Instantaneous drag coefficient $\left(C_{d}\right)$ versus nondimensional time $(\tau)$ for $\operatorname{Re}=$ 3000 following Equation 7.2, (+): present simulation, $(\times)$ : Akbari and Price [68].

\subsection{Results and discussion}

Two flat plates with $t / c=10 \%$ and chord-length $c$ perform harmonic pitching oscillations according to Equation 2. The airfoils are in a tandem configuration and oscillate with the same phase angle (in-phase oscillations). The distance between the airfoil's centers is $4 c$. The so-called right airfoil oscillates in the downstream wake of the so-called left airfoil. In the following paragraphs, the difference between the unsteady forces of the left and right airfoils in some investigated cases is compared. Then, the effects of $\theta$ and Re on the instantaneous fluid forces and moment are discussed. The amplitude of pitching oscillations $(\theta)$ is varied for $\theta=15^{\circ}, 30^{\circ}$ and $45^{\circ}$, and Re is varied for $\mathrm{Re}=50,75$ and 100 . For the sake of brevity, comparison between the $C_{l}$ and $C_{d}$ of the two airfoils is conducted for $\theta=15^{\circ}, 30^{\circ}$ and $45^{\circ}$ when $\operatorname{Re}=100$. Figure 7.4 shows $C_{l}$ and $C_{d}$ of the two airfoils when $\operatorname{Re}=100$ and $\theta=15^{\circ}$. As can be seen, both lift and drag coefficients have a sinusoidal pattern and the difference between the force coefficients is small. The discrepancy is mainly around $C_{l_{\max }}$ and $C_{d_{\max }}$. The difference between the forces coefficients (Figure 7.5) turns out to be larger at $\mathrm{Re}=100$ and $\theta=30^{\circ}$, and it is not just limited to the close proximity of the maximum lift and drag 
coefficients. It should be mentioned that $C_{l}$ oscillates with the forcing reduced frequency ( $k$ $\left.=\frac{\pi f c}{U_{\infty}}\right)$, but $C_{d}$ oscillates with twice the frequency, as expected. Increasing $\theta$ from $30^{\circ}$ to $45^{\circ}$, increases the observed difference between the lift and drag coefficients of the left and right airfoils (Figure 7.6). This is mainly due to the stronger vortices generated around each airfoil and the interaction between the vortical patterns of two airfoils. It should also be mentioned that the difference is larger during downstorkes, i.e., $0.5<\tau$, Fig. 7.6.

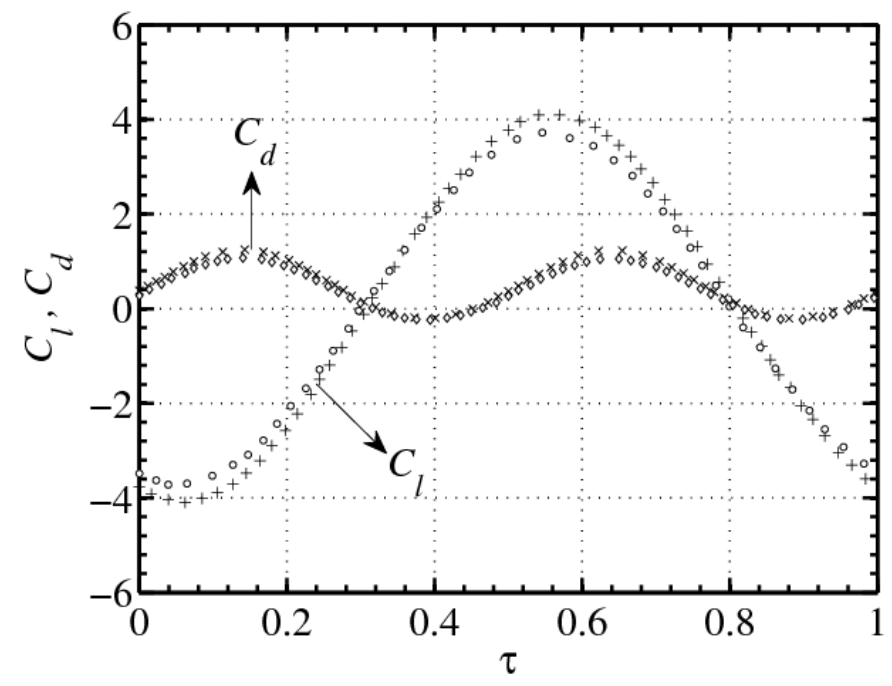

Figure 7.4: Instantaneous lift and drag coefficients $\left(C_{l}\right.$ and $\left.C_{d}\right)$ versus nondimensional time $(\tau)$ for $\operatorname{Re}=100$ and $\theta=15^{\circ},(+$ and $\times)$, left airfoil; (o and $\left.\diamond\right)$, right airfoil.

\subsubsection{Effect of amplitude of pitching oscillations}

The effect of the amplitude of pitching oscillations $(\theta)$ is investigated for $\theta=15^{\circ}, 30^{\circ}$ and $45^{\circ}$. Based on the following figures and explanations, it is found that the amplitude of pitching oscillation affects the instantaneous forces and moments both quantitatively and qualitatively, and is of primary importance to fluid dynamic characteristics of pitching airfoils. Figure 7.7 shows $C_{l}$ versus non-dimensional time of the right airfoil when $\operatorname{Re}=50$ and $\theta=15^{\circ}, 30^{\circ}$ and $45^{\circ}$. As can be seen, the maximum lift coefficient $\left(C_{l_{\max }}\right)$ is increased when $\theta$ increases. The lift coefficients at $\theta=15^{\circ}$ have the least fluctuations which means the strength of the generated vortices is less than those at $\theta=30^{\circ}$ and $45^{\circ}$, and lower vortex 


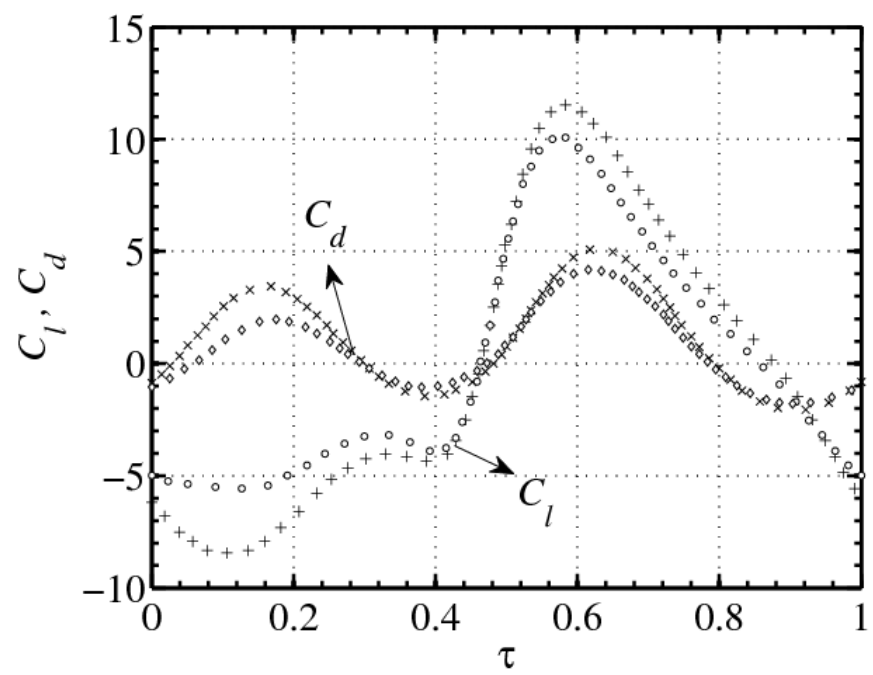

Figure 7.5: Instantaneous lift and drag coefficients $\left(C_{l}\right.$ and $\left.C_{d}\right)$ versus nondimensional time $(\tau)$ for $\operatorname{Re}=100$ and $\theta=30^{\circ},(+$ and $\times)$, left airfoil; (o and $\left.\diamond\right)$, right airfoil.

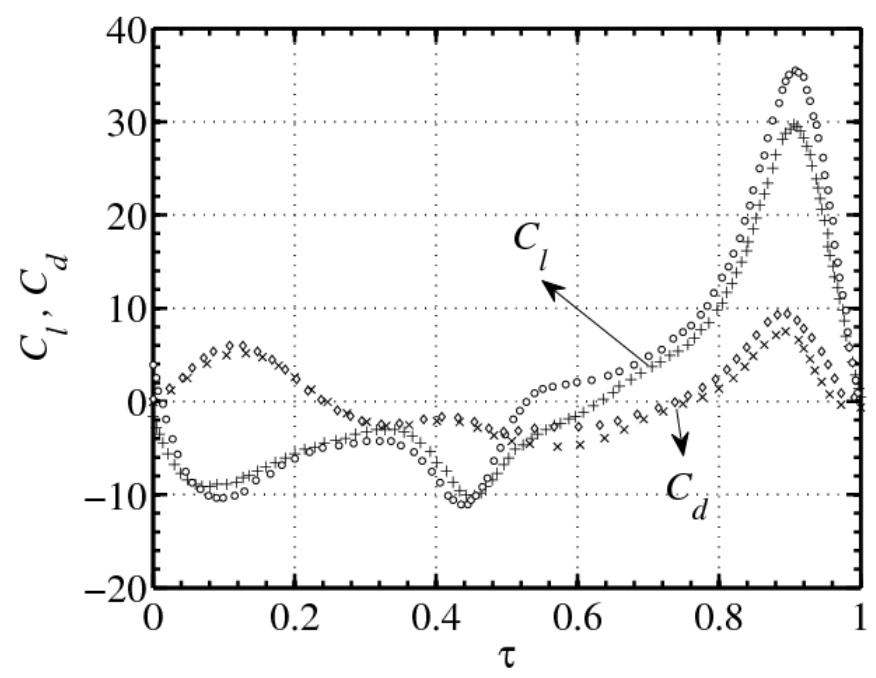

Figure 7.6: Instantaneous lift and drag coefficients $\left(C_{l}\right.$ and $\left.C_{d}\right)$ versus nondimensional time $(\tau)$ for $\operatorname{Re}=100$ and $\theta=45^{\circ},(+$ and $\times)$, left airfoil; $(o$ and $\diamond)$, right airfoil.

interaction has occurred between two airfoils. Increasing $\theta$ causes more fluctuations in the lift coefficients at $\theta=30^{\circ}$ and $45^{\circ}$.

Table 7.1 shows the magnitude of $\bar{C}_{l}$ and $\bar{C}_{d}$ for the up- and down-strokes for each investigated $\theta$ at $\operatorname{Re}=50$.

Figure 7.8 shows $C_{l}$ versus non-dimensional time when $\operatorname{Re}=75$ and $\theta=15^{\circ}, 30^{\circ}$ and 


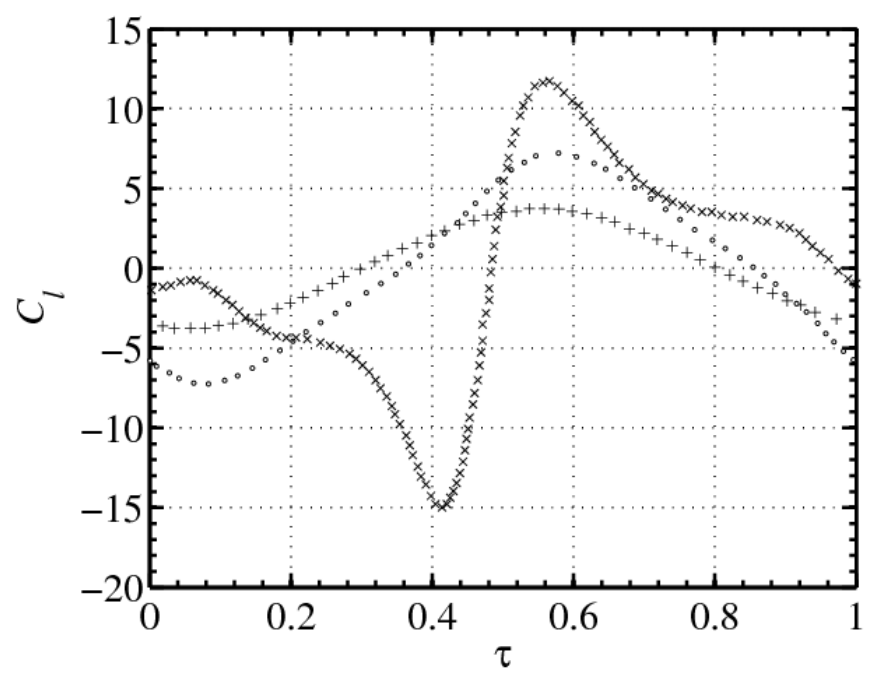

Figure 7.7: Instantaneous lift coefficient $\left(C_{l}\right)$ versus nondimensional time $(\tau)$ for right airfoil, $\operatorname{Re}=50,(+), \theta=15^{\circ} ;(\mathrm{o}), \theta=30^{\circ} ;(\times), \theta=45^{\circ}$.

Table 7.1: Mean lift and drag coefficients $\left(\bar{C}_{l}\right.$ and $\left.\bar{C}_{d}\right)$ for the considered amplitudes of pitching oscillations $(\theta)$ when $\operatorname{Re}=50$.

\begin{tabular}{ccc}
\hline \hline$\theta$ & Upstroke & Downstroke \\
\hline $15^{\circ}$ & $\left|\bar{C}_{l}\right|=0.696,\left|\bar{C}_{d}\right|=0.532$ & $\left|\bar{C}_{l}\right|=0.88,\left|\bar{C}_{d}\right|=0.524$ \\
$30^{\circ}$ & $\left|\bar{C}_{l}\right|=2.226,\left|\bar{C}_{d}\right|=0.788$ & $\left|\bar{C}_{l}\right|=1.723,\left|\bar{C}_{d}\right|=0.687$ \\
$45^{\circ}$ & $\left|\bar{C}_{l}\right|=5.932,\left|\bar{C}_{d}\right|=0.148$ & $\left|\bar{C}_{l}\right|=5.731,\left|\bar{C}_{d}\right|=1.708$ \\
\hline
\end{tabular}

$45^{\circ}$. Increasing $\theta$ increases $C_{l_{\max }}$ noticeably. This increase is associated with significant lag. That is, $C_{l_{\max }}$ at $\theta=15^{\circ}, 30^{\circ}$ and $45^{\circ}$ is obtained at $\tau=0.54,0.6$ and 0.9 , respectively. It should also be mentioned that the observed valley $(\tau=0.43)$ in Fig. 7.8 at $\theta=45^{\circ}$ corresponds to the so-called figure-of-eight phenomenon [10], after which the upstroke lift coefficients are bigger than those of the downstroke (at the same angle of attack). Fig. 7.9 shows the drag coefficient $\left(C_{d}\right)$ versus $\tau$ for $\operatorname{Re}=75$ and $\theta=15^{\circ}, 30^{\circ}$ and $45^{\circ}$. As shown, $\theta$ has significant effects on the magnitude and behavior of drag coefficients.

Table 7.2 shows the magnitude of $\bar{C}_{l}$ and $\bar{C}_{d}$ for the up- and down-strokes for each investigated $\theta$ at $\operatorname{Re}=75$. It can be seen that higher $\theta$ increases $\bar{C}_{l}$ and $\bar{C}_{d}$ in each half stroke.

Figs. 7.10- 7.12 show $C_{l}, C_{d}$ and $C_{m}$, respectively, versus nondimensional time when Re 


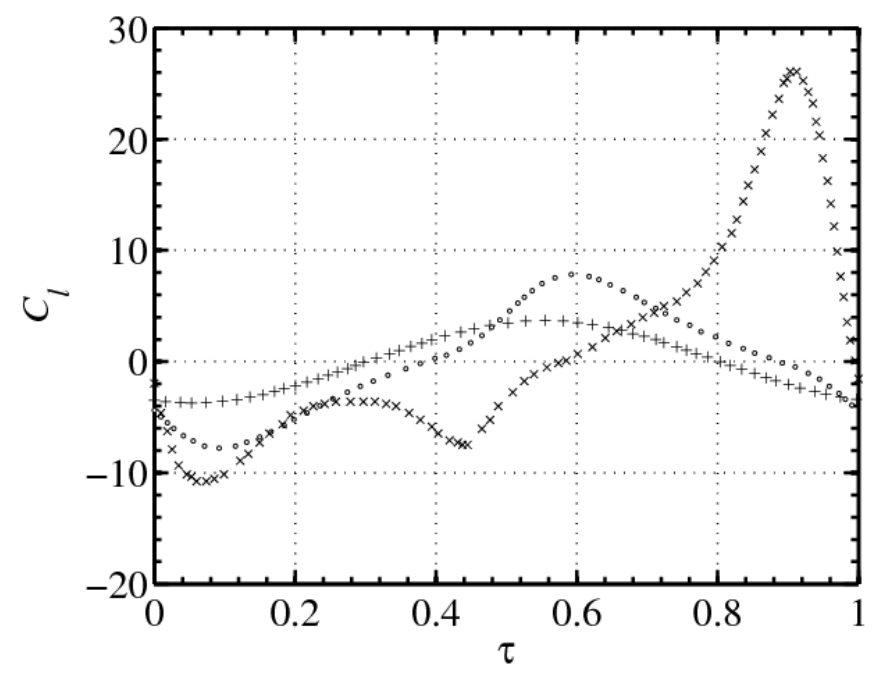

Figure 7.8: Instantaneous lift coefficient $\left(C_{l}\right)$ versus nondimensional time $(\tau)$ for right airfoil, $\operatorname{Re}=75:(+), \theta=15^{\circ} ;(\mathrm{o}), \theta=30^{\circ} ;(\times), \theta=45^{\circ}$.

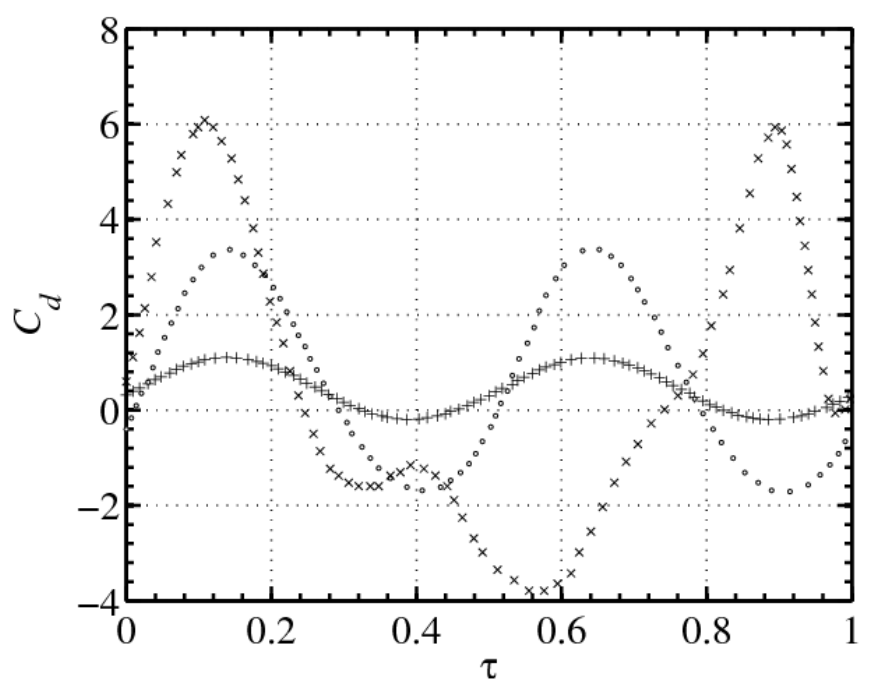

Figure 7.9: Instantaneous drag coefficient $\left(C_{d}\right)$ versus nondimensional time $(\tau)$ for right airfoil, $\operatorname{Re}=75:(+), \theta=15^{\circ} ;(\mathrm{o}), \theta=30^{\circ} ;(\times), \theta=45^{\circ}$.

Table 7.2: Mean lift and drag coefficients $\left(\bar{C}_{l}\right.$ and $\left.\bar{C}_{d}\right)$ for the considered amplitudes of pitching oscillations $(\theta)$ when $\operatorname{Re}=75$.

\begin{tabular}{ccc}
\hline \hline$\theta$ & Upstroke & Downstroke \\
\hline $15^{\circ}$ & $\left|\bar{C}_{l}\right|=0.798,\left|\bar{C}_{d}\right|=0.444$ & $\left|\bar{C}_{l}\right|=0.631,\left|\bar{C}_{d}\right|=0.428$ \\
$30^{\circ}$ & $\left|\bar{C}_{l}\right|=3.161,\left|\bar{C}_{d}\right|=0.706$ & $\left|\bar{C}_{l}\right|=2.952,\left|\bar{C}_{d}\right|=0.748$ \\
$45^{\circ}$ & $\left|\bar{C}_{l}\right|=6.268,\left|\bar{C}_{d}\right|=1.233$ & $\left|\bar{C}_{l}\right|=10.743,\left|\bar{C}_{d}\right|=1.003$ \\
\hline
\end{tabular}


$=100$ and $\theta=15^{\circ}, 30^{\circ}$ and $45^{\circ}$. Similar effects as those seen in the previous figures are observed here.

Table 7.3 shows the magnitude of $\bar{C}_{l}$ and $\bar{C}_{d}$ for the up- and down-strokes for each

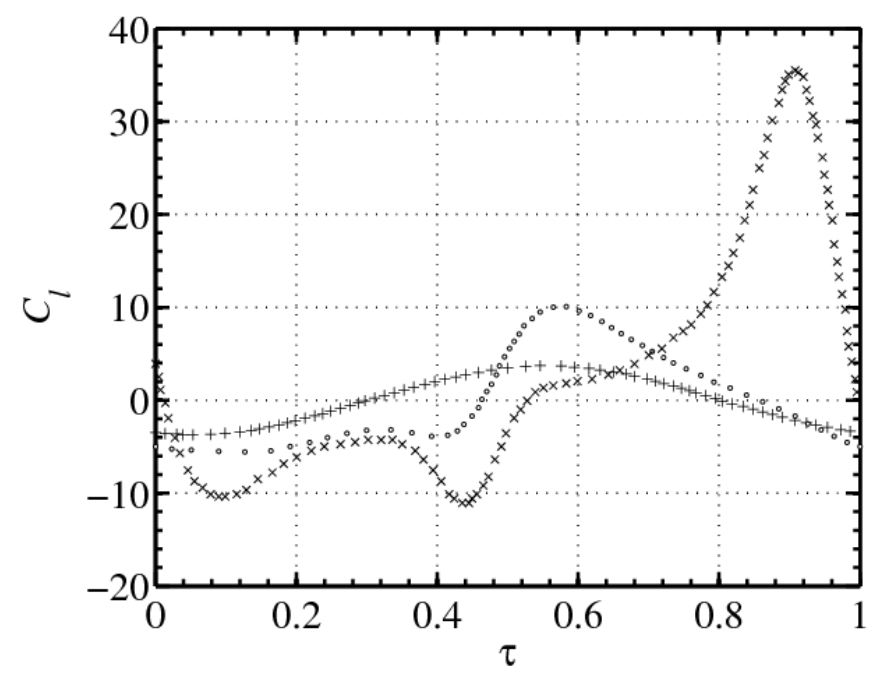

Figure 7.10: Instantaneous lift coefficient $\left(C_{l}\right)$ versus nondimensional time $(\tau)$ for right airfoil, $\operatorname{Re}=100:(+), \theta=15^{\circ} ;(\mathrm{o}), \theta=30^{\circ} ;(\times), \theta=45^{\circ}$.

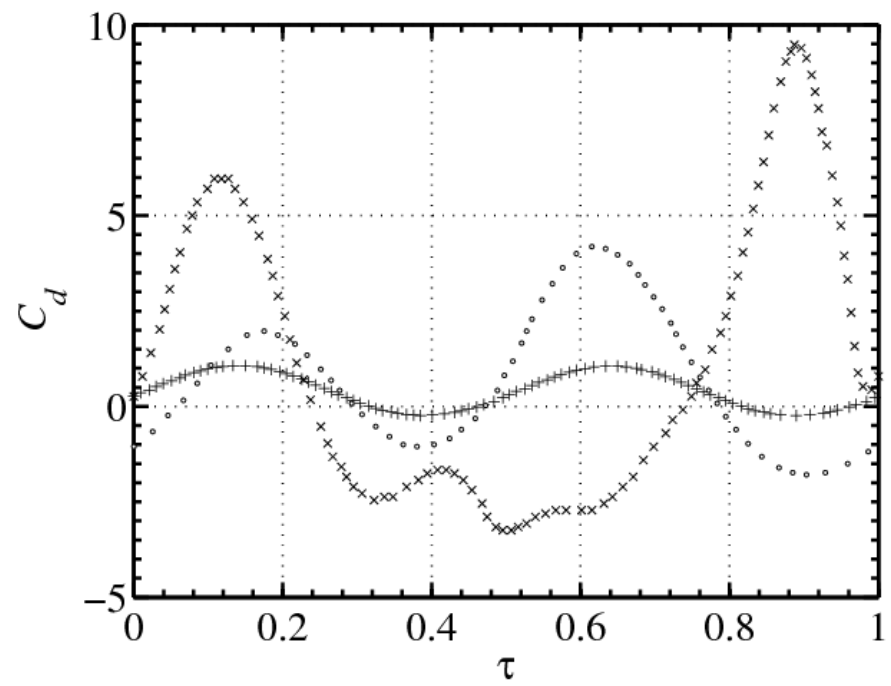

Figure 7.11: Instantaneous drag coefficient $\left(C_{d}\right)$ versus nondimensional time $(\tau)$ for right airfoil, $\operatorname{Re}=100:(+), \theta=15^{\circ} ;(\mathrm{o}), \theta=30^{\circ} ;(\times), \theta=45^{\circ}$.

investigated $\theta$ at $\operatorname{Re}=100$. 


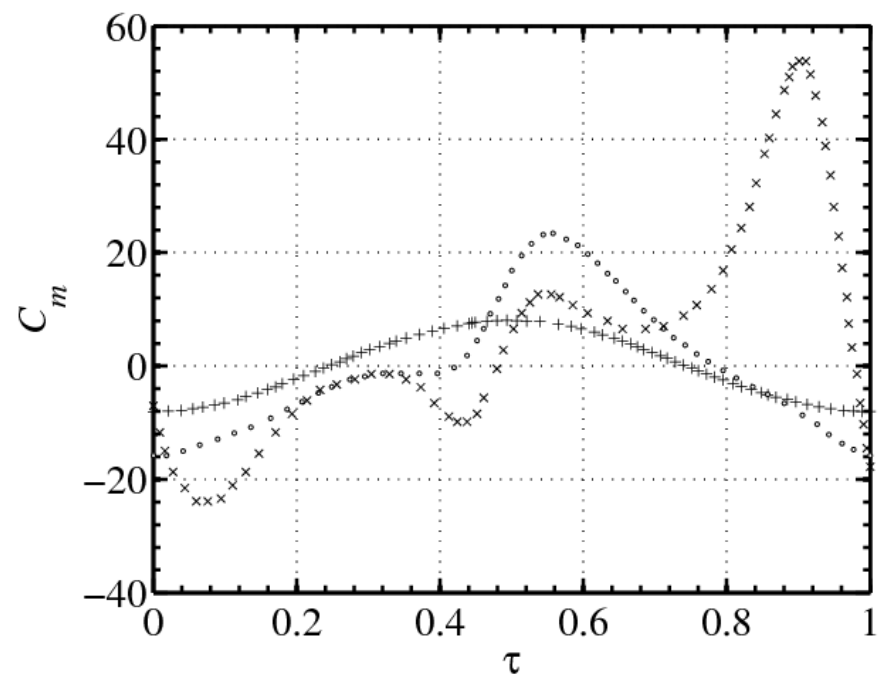

Figure 7.12: Instantaneous pitching moment coefficient $\left(C_{m}\right)$ versus nondimensional time $(\tau)$ for right airfoil, $\operatorname{Re}=100:(+), \theta=15^{\circ} ;(\mathrm{o}), \theta=30^{\circ} ;(\times), \theta=45^{\circ}$.

Table 7.3: Mean lift and drag coefficients $\left(\bar{C}_{l}\right.$ and $\left.\bar{C}_{d}\right)$ for the considered amplitudes of pitching oscillations $(\theta)$ when $\operatorname{Re}=100$.

\begin{tabular}{ccc}
\hline \hline$\theta$ & Upstroke & Downstroke \\
\hline $15^{\circ}$ & $\left|\bar{C}_{l}\right|=0.821,\left|\bar{C}_{d}\right|=0.405$ & $\left|\bar{C}_{l}\right|=0.607,\left|\bar{C}_{d}\right|=0.419$ \\
$30^{\circ}$ & $\left|\bar{C}_{l}\right|=2.346,\left|\bar{C}_{d}\right|=0.276$ & $\left|\bar{C}_{l}\right|=4.013,\left|\bar{C}_{d}\right|=1.377$ \\
$45^{\circ}$ & $\left|\bar{C}_{l}\right|=6.347,\left|\bar{C}_{d}\right|=0.903$ & $\left|\bar{C}_{l}\right|=15.388,\left|\bar{C}_{d}\right|=2.545$ \\
\hline
\end{tabular}

\subsubsection{Effect of Reynolds number}

The effect of Reynolds number (Re) on the unsteady forces is investigated for $\mathrm{Re}=50$, 75 and 100. Based on the following figures and explanations, it is found that Re is of secondary importance to the fluid dynamic characteristics of a pitching airfoil compared to the importance of the amplitude of pitching oscillations.

Figure 7.13 shows $C_{l}$ versus $\tau$ for $\theta=30^{\circ}$ and $\operatorname{Re}=50,75$ and 100. As can be seen, Re does not change the magnitude and the oscillatory pattern of $C_{l}$ noticeably, when compared to the effects of the amplitude of pitching oscillations. The drag coefficient of the right airfoil is also shown in Fig. 7.14. Increasing Re from 50 to 75 does not have any noticeable effect on $C_{d}$, but increasing Re from 75 to 100 changes both first and second $C_{d_{\max }}$. These changes are associated with lead/lag, Fig. 7.14. 
Table 7.4 shows the magnitude of $\bar{C}_{l}$ and $\bar{C}_{d}$ for the up- and down-strokes for each

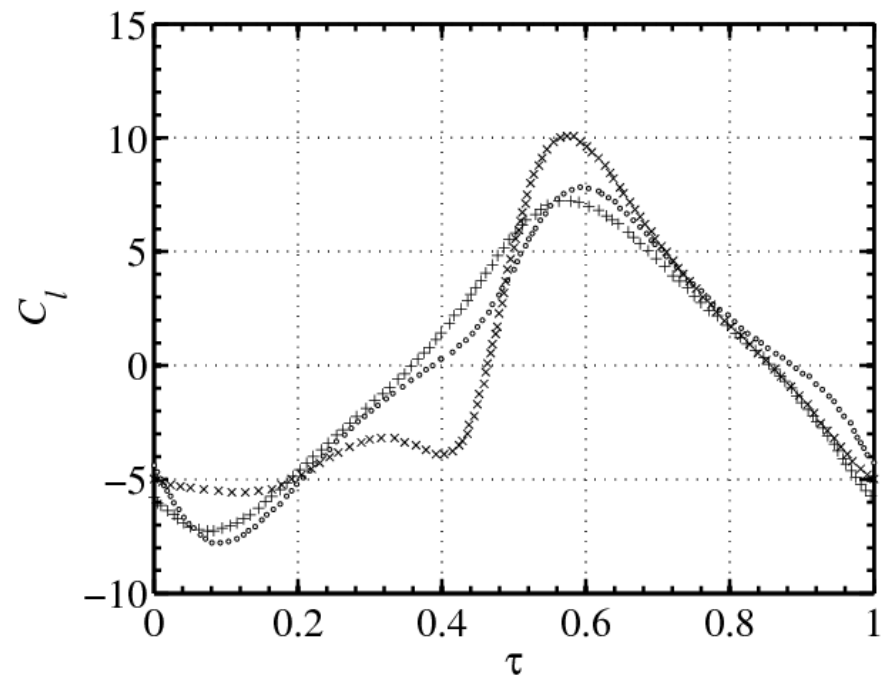

Figure 7.13: Instantaneous lift coefficient $\left(C_{l}\right)$ versus nondimensional time $(\tau)$ for right airfoil when $\theta=30^{\circ}$ : $(+), \operatorname{Re}=50 ;(\mathrm{o}), \operatorname{Re}=75 ;(\times), \operatorname{Re}=100$.

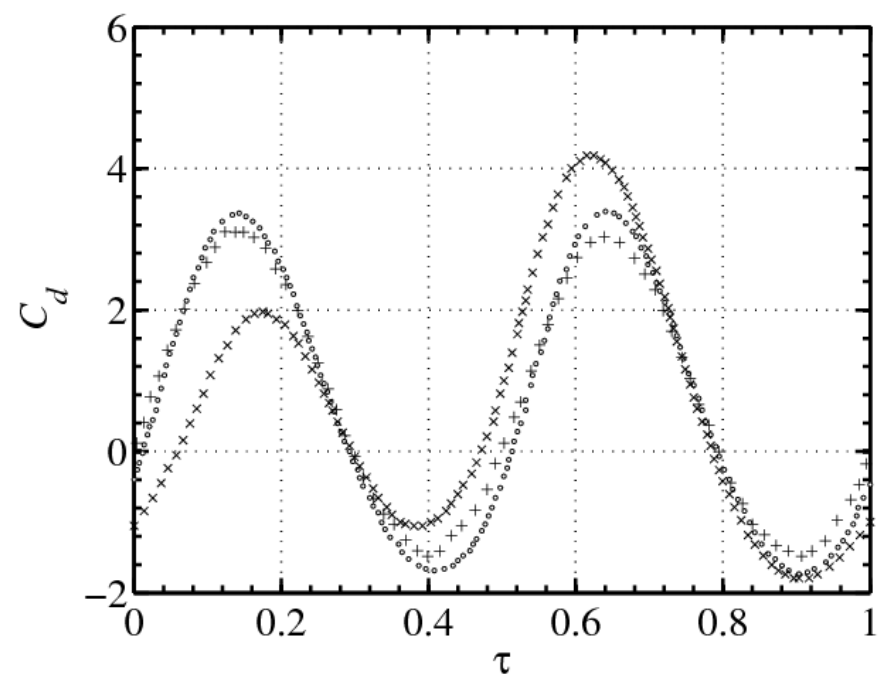

Figure 7.14: Instantaneous drag coefficient $\left(C_{d}\right)$ versus nondimensional time $(\tau)$ for right airfoil when $\theta=30^{\circ}$ : (+), $\operatorname{Re}=50 ;(\mathrm{o}), \operatorname{Re}=75 ;(\times), \operatorname{Re}=100$.

investigated Re at $\theta=30^{\circ}$.

Figures 7.15 and 7.16 show $C_{l}$ and $C_{d}$ versus $\tau$, respectively, for $\theta=45^{\circ}$ and $\operatorname{Re}=50$, 75 and 100. As shown, Re does not have any effect on the pattern of the unsteady force 
Table 7.4: Mean lift and drag coefficients $\left(\bar{C}_{l}\right.$ and $\left.\bar{C}_{d}\right)$ for the considered Re when $\theta=30^{\circ}$.

\begin{tabular}{ccc}
\hline \hline $\operatorname{Re}$ & Upstroke & Downstroke \\
\hline 50 & $\left|\bar{C}_{l}\right|=2.226,\left|\bar{C}_{d}\right|=0.788$ & $\left|\bar{C}_{l}\right|=1.723,\left|\bar{C}_{d}\right|=0.687$ \\
75 & $\left|\bar{C}_{l}\right|=3.161,\left|\bar{C}_{d}\right|=0.706$ & $\left|\bar{C}_{l}\right|=2.952,\left|\bar{C}_{d}\right|=0.748$ \\
100 & $\left|\bar{C}_{l}\right|=2.346,\left|\bar{C}_{d}\right|=0.276$ & $\left|\bar{C}_{l}\right|=4.013,\left|\bar{C}_{d}\right|=1.377$ \\
\hline
\end{tabular}

coefficients, but it changes the magnitude of the maximum unsteady forces.

Table 7.5 shows the magnitude of $\bar{C}_{l}$ and $\bar{C}_{d}$ for the up- and down-strokes for each

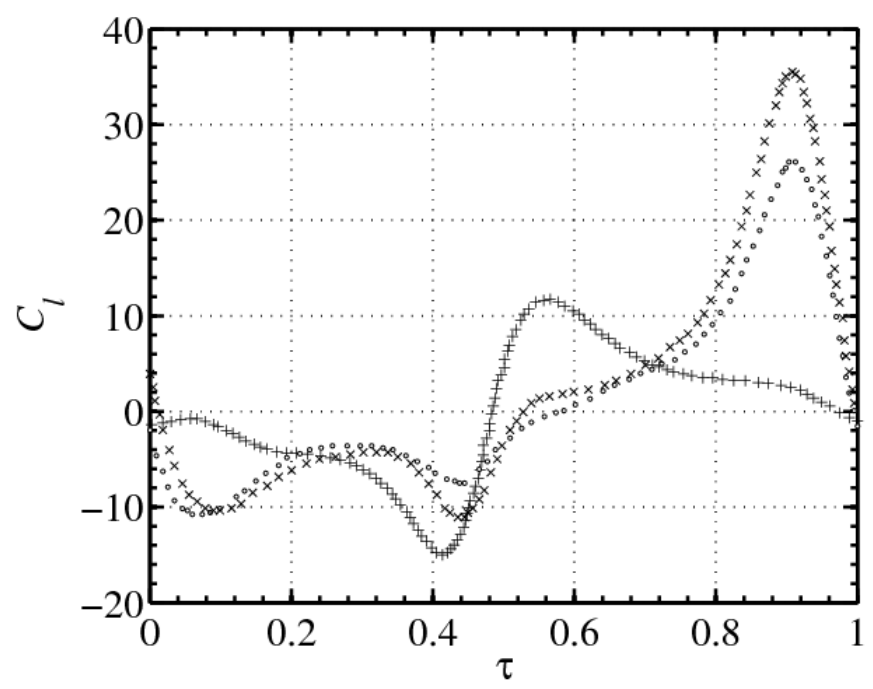

Figure 7.15: Instantaneous lift coefficient $\left(C_{l}\right)$ versus nondimensional time $(\tau)$ for right airfoil when $\theta=45^{\circ}:(+), \operatorname{Re}=50 ;(\mathrm{o}), \operatorname{Re}=75 ;(\times), \operatorname{Re}=100$.

investigated Re at $\theta=45^{\circ}$.

Table 7.5: Mean lift and drag coefficients $\left(\bar{C}_{l}\right.$ and $\left.\bar{C}_{d}\right)$ for the considered Re when $\theta=45^{\circ}$.

\begin{tabular}{ccc}
\hline \hline Re & Upstroke & Downstroke \\
\hline 50 & $\left|\bar{C}_{l}\right|=5.932,\left|\bar{C}_{d}\right|=0.148$ & $\left|\bar{C}_{l}\right|=5.731,\left|\bar{C}_{d}\right|=1.708$ \\
75 & $\left|\bar{C}_{l}\right|=6.268,\left|\bar{C}_{d}\right|=1.233$ & $\left|\bar{C}_{l}\right|=10.743,\left|\bar{C}_{d}\right|=1.003$ \\
100 & $\left|\bar{C}_{l}\right|=6.347,\left|\bar{C}_{d}\right|=0.903$ & $\left|\bar{C}_{l}\right|=15.388,\left|\bar{C}_{d}\right|=2.545$ \\
\hline
\end{tabular}




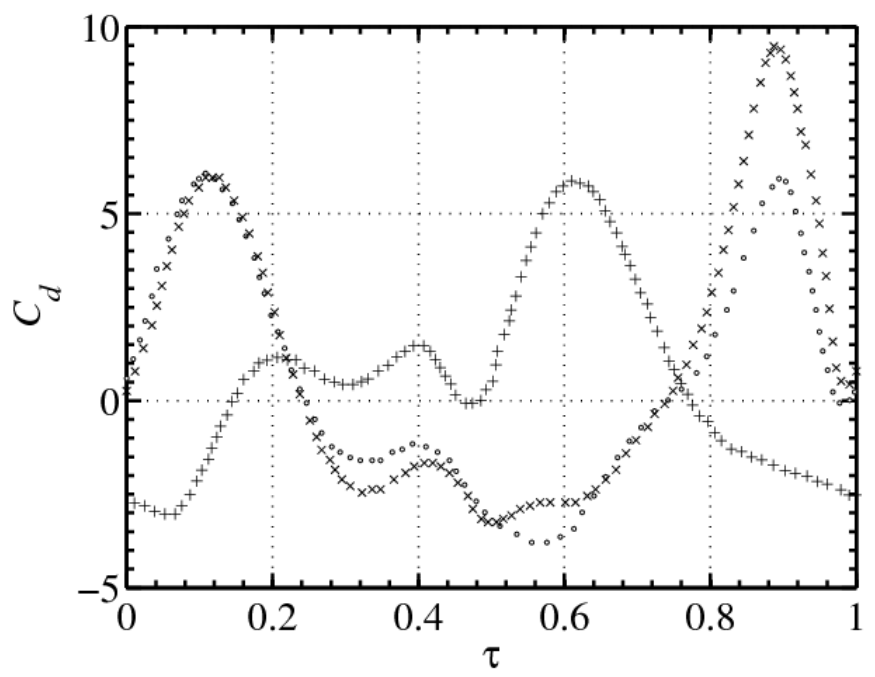

Figure 7.16: Instantaneous drag coefficient $\left(C_{d}\right)$ versus nondimensional time $(\tau)$ for right airfoil when $\theta=45^{\circ}$ : $(+), \operatorname{Re}=50 ;($ o), $\operatorname{Re}=75 ;(\times), \operatorname{Re}=100$.

\subsection{Summary and conclusion}

A numerical study is conducted to investigate the instantaneous unsteady forces of a pair of flat plates in LRN flow regime by extending GGI technique [132] to handle multi body rotations. The airfoils perform pitching oscillations with the same phase angle, and are in a tandem configuration. The simulations are based on NS equations and FVM method. As shown, the interaction between the two airfoil's vortices changes the magnitude of the unsteady forces. The effects of amplitude of pitching oscillations and Re on the lift, drag, and moment coefficients are also investigated. It is shown that the amplitude of pitching oscillation changes the instantaneous force coefficients both quantitatively and qualitatively. However, Re is shown to be of secondary importance compared to the amplitude of pitching oscillations. Re changes the magnitude of the force coefficients, but does not have any significant effect on the pattern of the calculated forces. In the following chapter, the force signatures of a pair of plunging airfoils are investigated. 


\section{Chapter 8}

\section{On the force signatures of a pair of plunging airfoils}

The unsteady aerodynamics of a pair of multi-plunging airfoils is studied using Computational Fluid Dynamics (CFD) based on the Finite Volume Method (FVM) and dynamic layering mesh motion algorithm. The two-dimensional unsteady, incompressible NavierStokes (N-S) equations are used as the governing equations while the thin ellipsoidal airfoils, commonly used in Micro Aerial Vehicles (MAVs), perform harmonic plunging motion. The instantaneous lift and drag coefficients are examined in detail and the effects of Reynolds number, frequency and amplitude of oscillations, and the airfoils' centre-to-centre spacing on the force coefficients are investigated. It is shown that the force coefficients of each of the plunging airfoils differ noticeably from those of a single plunging airfoil both quantitatively and qualitatively, showing the significance of the airfoil-airfoil interaction. It is also observed that the investigated parameters affect the magnitude and characteristics of the instantaneous lift and drag coefficients. There is an optimum frequency of oscillations, resulting in the highest thrust generation between the investigated frequencies. The amplitude of oscillations increases the aerodynamic performance by increasing the mean lift coefficient but decreasing the mean drag coefficient at the same time. Re effects on the lift coefficient 
are negligible; however, it is shown that increasing Re causes the airfoils to generate more thrust compared to the lower Reynolds numbers.

\subsection{Introduction}

In some applications, multi-plunging bodies exist where the simultaneous transverse oscillations change the aerodynamics and vortical patterns, compared to the characteristics of a single plunging body, and adds to the complexity of the corresponding fluid flow. Kaya et al. [60] showed that the aerodynamics of multi-flapping airfoils is significantly different from single cases in terms of the generated thrust. The tube bundle of a heat exchanger, counterrotating propellers, wings of a MAV or blades of a wind turbine are some examples in which multi-plunging oscillations could occur. To the best of author's knowledge, the studies on multi-plunging cylinders and airfoils are scarce and those existing are mostly on tandem and staggered cylinder arrangements. Mahir and Rockwell [61] and [62] experimented on the wakes of tandem/staggered cylinder arrangements at Re $=160$. In their study, the cylinder system was subjected to forced excitation with different frequencies and phase angles between the oscillating cylinders. They observed that the generated vortical patterns and their

mechanisms vary significantly with respect to the excitation frequency. Assi et al. [63] also carried out experiments of two tandem cylinders under flow-induced oscillations. Chern et al. [96] simulated the flow patterns of a pair of side-by-side squared cylinders. They used harmonic oscillatory flow around cylinders and investigated the generated flow patterns. They evaluated the effects of Reynolds number and cylinder gap spacing on the surrounding flow and concluded that Re has fewer effects on the flow physics than does the gap spacing. Lee et al. [97] used immersed boundary method to simulate the flow around two side-by-side oscillating cylinders at $\mathrm{Re}=185$. They investigated the effect of the gap between the cylinders and the frequency of oscillations on the wake topology and instantaneous force coefficients, and concluded that two oscillating cylinders have characteristics of two stationary cylinders 
and a single oscillating cylinder. Mital and Kumar [98] used a Finite Element formulation to study the flow-induced oscillations between two tandem and staggered cylinders in low Reynolds number flow. They used cylinder spacing such that the flow-induced oscillations occurred in the wake-inference regime, where the cylinders are within close distance and the wake of one cylinder affects the other cylinder flow patterns. They observed that the downstream cylinder undergoes very large amplitude oscillations. The numerical simulations of Papaioannou et al. [99] showed the effect of spacing in a tandem arrangement under vortexinduced oscillations. They observed that the response region of the upstream cylinder is widened when spacing decreases. They also showed that the amplitude of vortex-induced oscillations depends on the spacing. Prasanth and Mittal [100] used FEM to study the 2-D flow-induced oscillations of two tandem cylinders at LRN regime. They allowed the cylinders to move in both in-flow and cross-flow directions. Jester and Kallinderis [102] numerically investigated the incompressible flow around transversally oscillating cylinder pairs, in both tandem and side-by-side arrangements, and explored the effect of phase angle on the wake structures of the oscillating bodies.

The present study is conducted to examine the effects of the important governing flow and system parameters on a pair of plunging airfoils at LRN regime. Although there are some limited studies on pairs of plunging cylinders, due attention has not been paid to the airfoils. One example of a pair of plunging airfoils is in the MAVs. The present study reveals the importance of the airfoil-airfoil interactions, and shows how this interaction affects the instantaneous lift and drag coefficients. Moreover, by conducting a parametric study, the effect of the governing parameters on the force benchmarks are investigated. The computations in this study are performed in OpenFOAM ${ }^{\circledR}$. 


\subsection{Numerical simulation method}

The flow field of a plunging airfoil is governed by the unsteady incompressible 2-D NavierStokes (N-S) equations. The Reynolds number (Re) defined in Equations 3.10 is based on the freestream velocity $\left(U_{\infty}\right)$, as shown in Fig. 8.1.

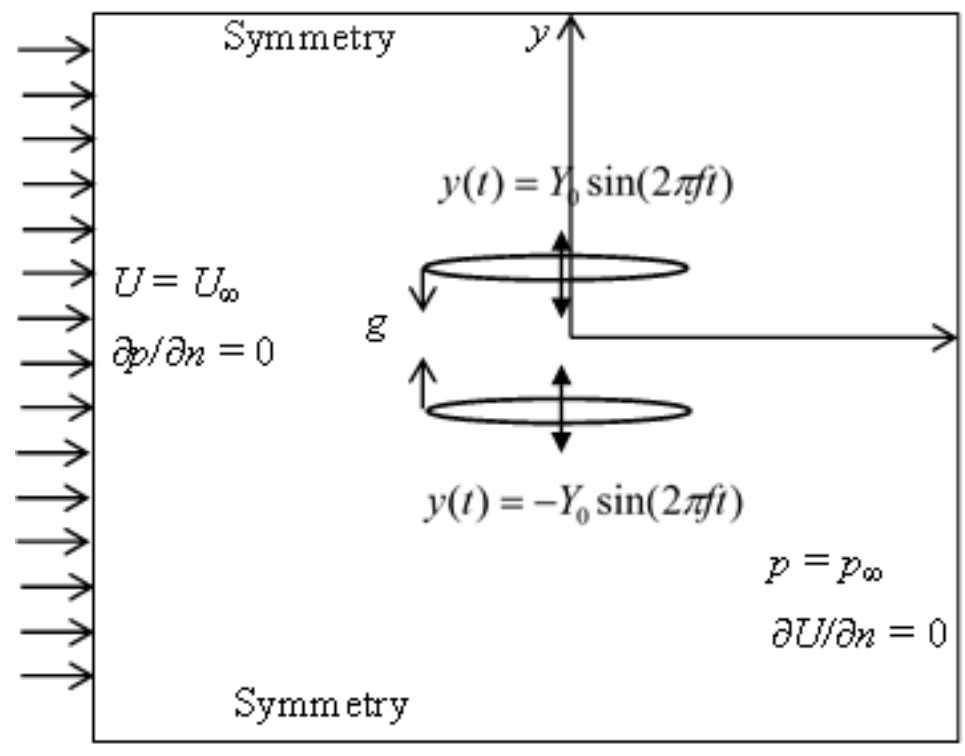

Figure 8.1: Schematic of the plunging airfoils and the computational domain.

The aerodynamic simulation of a pair of plunging ellipsoidal airfoils is performed using FVM and N-S equations. The computations utilize a 2nd-order central differencing scheme for convective and diffusive terms and a 2nd-order Euler implicit scheme for temporal discretizations. The resulting linear system of equations is treated with Preconditioned Conjugate Gradient (PCG) solvers, and the Semi-Implicit Method for Pressure Linked Equations (SIMPLE) algorithm is used for the pressure-velocity coupling. The computations are carried out in OpenFOAM ${ }^{\circledR}$. The equations of motion for the upper and lower airfoils are 
as the following:

$$
\begin{aligned}
& y_{u p}=Y_{0} \sin (2 \pi f t), \\
& y_{d n}=-Y_{0} \sin (2 \pi f t),
\end{aligned}
$$

where $y_{u p}(t)$ and $y_{d n}(t)$ represent the variation of the upper and lower airfoils' vertical position, respectively, $Y_{0}$ and $f$ are the amplitude and frequency of plunging oscillations, respectively.

In the present analysis, the effects of several flow and system parameters on the lift $\left(C_{l}\right)$ and drag $\left(C_{d}\right)$ coefficients of the upper and lower airfoils oscillating according to Equations 8.1 are investigated. The calculation of $C_{l}$ and $C_{d}$ is based on $F_{y}$ (fluid force on the airfoil in $y$ direction) and $F_{x}$ (fluid force on the airfoil in $x$ direction), respectively, according on the following equations:

$$
C_{l}=\frac{F_{y}}{\frac{1}{2} \rho U^{2} S} ; \quad C_{d}=\frac{F_{x}}{\frac{1}{2} \rho U^{2} S}
$$

where $\rho$ is the fluid density, $S=c$ and $U=\sqrt{U_{\infty}^{2}+\dot{y}_{\max }^{2}}$.

\subsection{Evaluation of the numerical approach}

At first, the effects of grid size and temporal resolution on the calculated lift and drag coefficients are examined in detail, and the mesh and time step are sufficiently refined such that the simulations are independent of the mesh size and time step resolutions. The sensitivity analyses presented in Figs. 8.2(a) and 8.2(b) correspond to $g=1.5, f=0.25, Y_{0}=0.5 c$ and Re $=75$, and a plunging motion defined according to Equations 8.1. As the lift coefficients of both the upper and lower airfoils are similar, the lift coefficient of the upper airfoil is only 
shown in Figs. 8.2(a) and 8.2(b) in a period. First, the grid sensitivity is investigated using 25, 50 and $75\left(\times 10^{3}\right)$ cells and 2000 time steps per plunging cycle. The results show that the difference between the instantaneous and mean lift and drag coefficients of 25 and 50 $\left(\times 10^{3}\right)$ cell cases are negligible. The time step is also varied for 1000, 2000 and 4000 time steps within a cycle having $50 \times 10^{3}$ cells. The results show that the difference between the lift coefficients of 2000 and 4000 time step cases is negligible. Therefore, the computations are done using $50 \times 10^{3}$ cells and 2000 time steps.
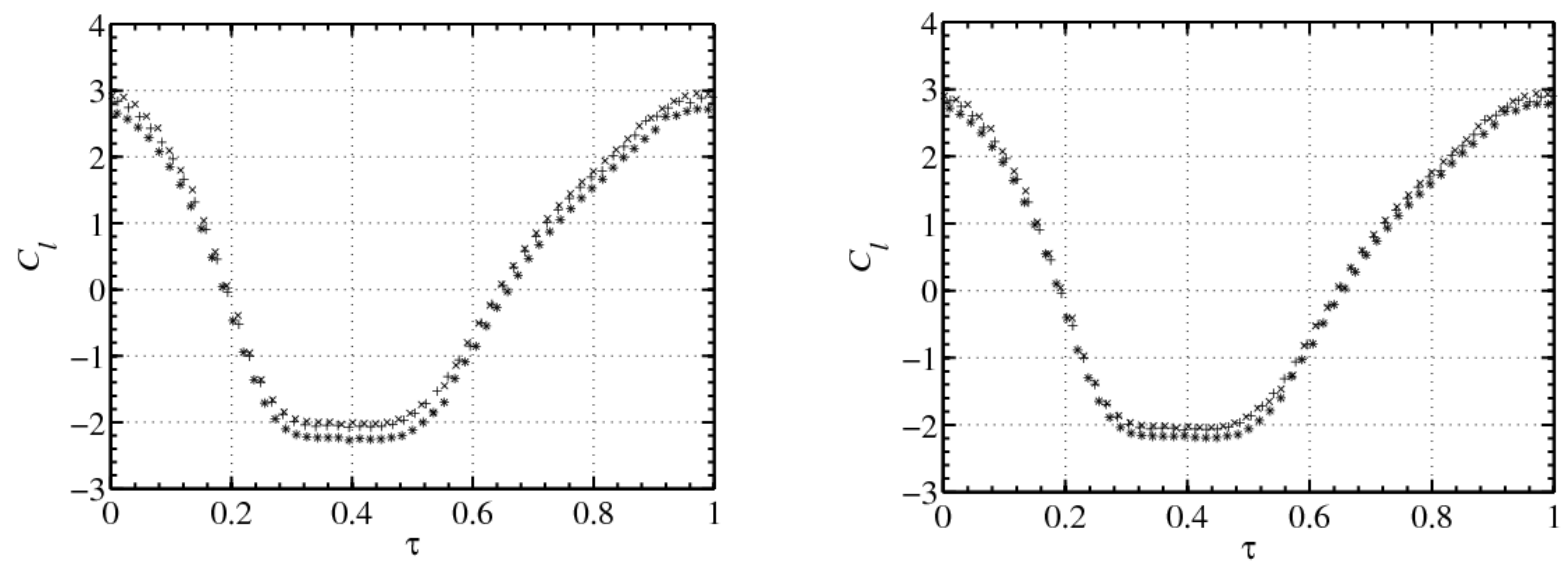

Figure 8.2: Instantaneous lift coefficient $\left(C_{l}\right)$ versus nondimensional time $(\tau)$ for $g=1.5, f$ $=0.25, Y_{0}=0.5$ and $\operatorname{Re}=75$, (a): (*) $25 \times 10^{3},(+) 50 \times 10^{3},(\times) 75 \times 10^{3}$ cell; (b): (*) 1000, (+) 2000, (×) 4000 time steps within a cycle.

Next, the accuracy of the dynamic layering mesh motion algorithm [141] is investigated. To do so, the ellipsoidal airfoil is forced to plunge according to $y(t)=Y_{0} \sin (2 \pi f t)$ with no freestream velocity. The same case with the same number of cells $\left(50 \times 10^{3}\right)$ and 2000 time steps per cycle is also simulated using the Laplace motion algorithm [107] with $Y_{0}=0.5 c$ and $f=1 \mathrm{~Hz}$. Figure 8.3 shows the lift coefficients obtained from both mesh motion algorithms. As can be observed, there is a very good match between instantaneous $C_{l}$ values obtained from the dynamic layering method and those of Laplace smoothing algorithm.

The accuracy of the numerical method and the mesh motion algorithm is then assessed based on the data published in the literature, available from studies conducted on cylinders. 


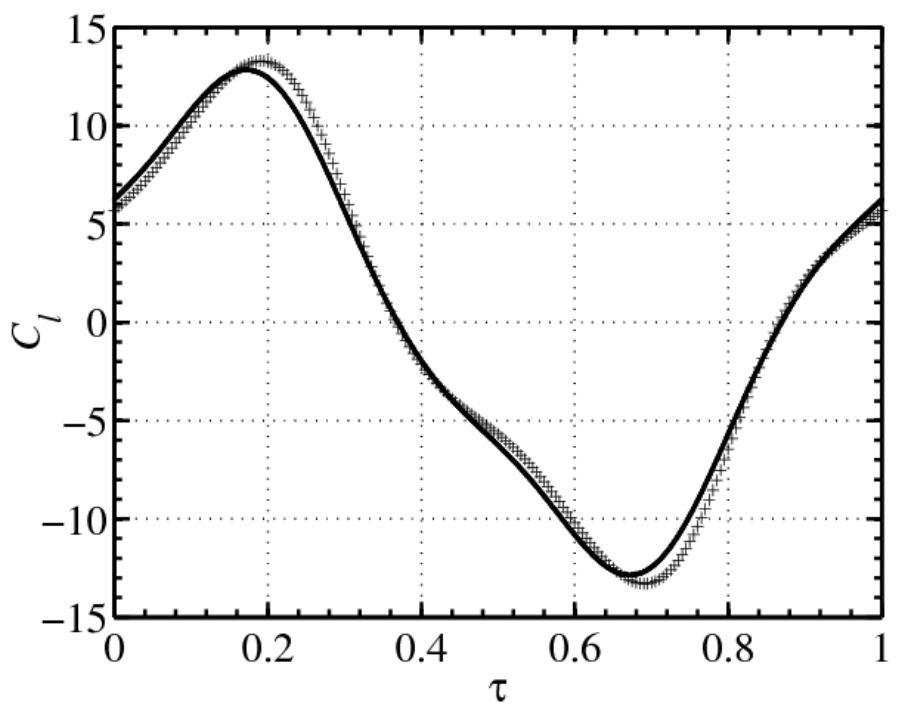

Figure 8.3: Instantaneous lift coefficient $\left(C_{l}\right)$ versus nondimensional time $(\tau)$ for a $2 \%$ thickness ellipsoidal airfoil plunging with $y(t)=Y_{0} \sin (2 \pi f t)$ with $Y_{0}=0.5 c$ and $f=1 ;(-)$, dynamic layering; (+), Laplace smoothing.

The side-by-side cylinder arrangement studied by Lee et al. [97] was examined for the validation purposes. They simulated the flow field of a pair of cylinders at $\operatorname{Re}=185$, performing plunging oscillations according to the following equations:

$$
\begin{aligned}
& y_{u p}=\frac{g_{0}}{2}+\frac{D}{2}+A_{e} \sin (2 \pi f t), \\
& y_{d n}=-\frac{g_{0}}{2}+\frac{D}{2}-A_{e} \sin (2 \pi f t),
\end{aligned}
$$

with $g_{0}=1.8, f=0.154, D=1$, and $A_{e}=0.2$.

Figures 8.4 and 8.5 show, respectively, the schematic of the computational domain and the computed vortical patterns in the downstream wake of the cylinders at $\tau \approx 0.5$.

Figure 8.6 shows the computed lift and drag coefficients of the cylinders in the simulation. The simulation results are in close agreement (less than $4 \%$ of difference) with those reported by Lee et al. [97]. 


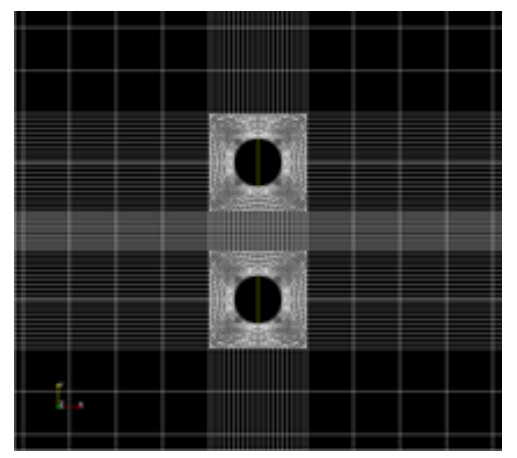

Figure 8.4: Schematic of cylinders and the computational domain.

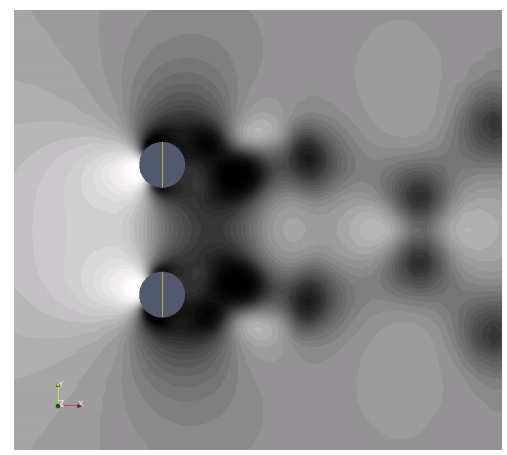

Figure 8.5: Vortical pattern in the downstream wake of the side-by-side cylinders plunging according to Equations 8.3 at $\tau \approx 0.5$.

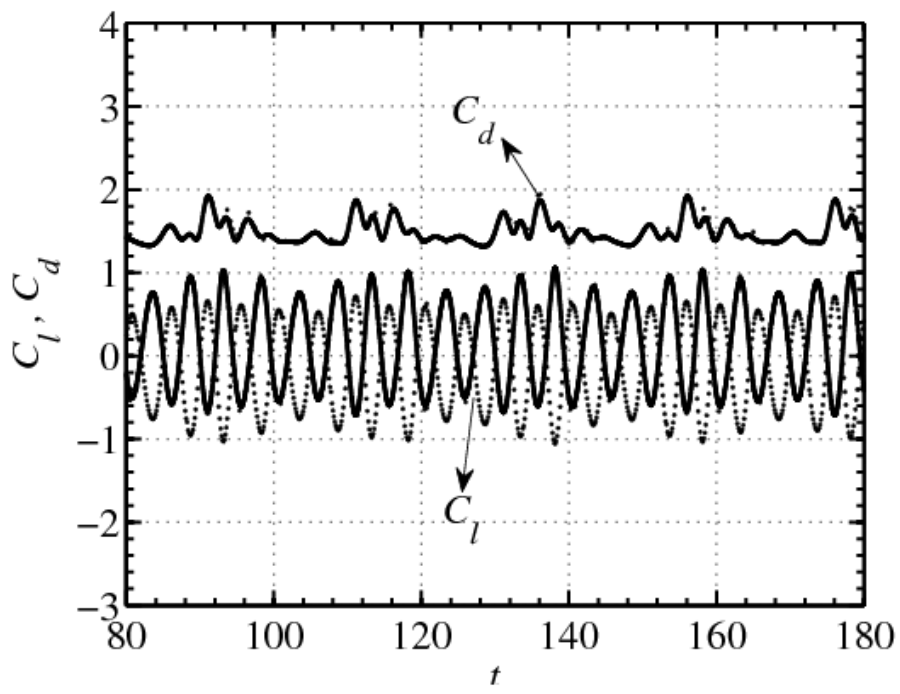

Figure 8.6: Instantaneous lift and drag coefficients $\left(C_{l}\right.$ and $\left.C_{d}\right)$ versus time for a pair of cylinders plunging according to Equations 8.3 with $g_{0}=1.8, f=0.154, D=1$, and $A_{e}=$ $0.2 ;(-)$, upper cylinder; (...), lower cylinder. 


\subsection{Results and discussion}

In what follows, the effects of the governing flow and system main parameters, namely $f, Y_{0}$, Re and airfoils' centre-to-centre spacing ( $g$, referred to the centre-to-centre spacing at the start time of the plunging motion) on the flow field of two ellipsoidal airfoils $(2 \%$ thickness) are investigated using N-S equations based on FVM and dynamic layering mesh motion algorithm [141]. The airfoils perform out-of-phase plunging oscillations according to Equations 8.1, also shown in Fig. 8.1. The airfoils plunge transversally with respect to the oncoming freestream flow. The lift and drag coefficients are computed, and the effects of the aforementioned parameters on the instantaneous force coefficients are investigated. Table 8.1 shows the parametric study settings used in each case.

Table 8.1: Parameter settings for the conducted case-studies

\begin{tabular}{ccc}
\hline \hline Parametric study & Variable parameters & Fixed parameters \\
\hline Effect of $\mathrm{Re}$ & $\mathrm{Re}=75,150,300,600$ & $f=0.25, Y_{0}=0.5 c, g=1.5 c$ \\
Effect of $f$ & $f=0.5,1,2 \mathrm{~Hz}$ & $Y_{0}=0.5 c, g=1.5 c, \mathrm{Re}=75$ \\
Effect of $Y_{0}$ & $Y_{0}=0.3 c, 0.5 c$ & $g=1.5 c, f=0.25, \mathrm{Re}=75$ \\
Effect of $Y_{0}$ & $Y_{0}=0.5 c, 0.7 c, 0.9 c$ & $g=3 c, f=0.25, \mathrm{Re}=75$ \\
Effect of $g$ & $g=1.5 c, 3 c$ & $f=0.25, \mathrm{Re}=75, Y_{0}=0.5 c$ \\
\hline
\end{tabular}

\subsubsection{Effect of the Reynolds number}

The effects of the Reynolds number (Re) on the lift and drag coefficients are investigated for the corresponding settings in Table 8.1. Based on the following observations and discussions, it is shown that the initially drag-producing airfoils at the lower considered $\operatorname{Re}(\operatorname{Re}=75$ and 150) are capable of generating thrust at the higher investigated Reynolds numbers (Re $=300$ and 600). Indeed, increasing Re causes the airfoil to generate more thrust. This thrust generation occurs mostly during the first quarter period when the airfoils move away. However, the airfoils always generate drag during the rest of the motion. The effects of Re on $C_{d}$ is mostly quantitative, whereas Re changes $C_{l}$ qualitatively, mostly during the second quarter period of the plunging cycle, when the airfoils start to move towards each other. 
Figure 8.7 shows $C_{l}$ versus $\tau$ at $\operatorname{Re}=75$ for the upper and lower airfoils as well as a single plunging airfoil under the same governing conditions. In the present analysis, the so-called upper and lower airfoils refer to the location of the airfoils in the computational domain according to Fig. 8.1. The calculated lift coefficients for upper and lower airfoils are observed to be equal in magnitude but opposite in sign. Also, as expected, both airfoils have the same drag coefficients, Fig. 8.8. As can be seen in Fig. 8.7, the minimum lift coefficient $\left(C_{l_{m i n}}\right)$ is almost the same as that of a single airfoil simulation; however, the lift coefficients decrease more rapidly for the upper airfoil simulation in the first half stroke $(\tau<0.5)$. It should also be mentioned that as the airfoils oscillate in out-of-phase plunging motion, the first half stroke corresponds to when the airfoils move away and the second half stroke $(0.5$ $<\tau)$ correspond to when the airfoils move towards each other. The magnitude of $C_{l_{\max }}$ is higher than $C_{l_{\min }}$, showing the effect of the airfoils' interaction. Comparing the $C_{l_{\max }}$ of the upper airfoil in Fig. 8.7 with that of the single airfoil also shows that the airfoils' interaction increases the maximum lift coefficient.

Fig. 8.8 shows $C_{d}$ versus $\tau$ at $\mathrm{Re}=75$ for the upper and lower and the single plunging airfoils under the same governing conditions. The $C_{d_{\max }}$ is negligibly changed when those of the single and multi-plunging airfoils are compared, but the minimum drag coefficients are obtained with lead/lag and considerably different magnitudes for single and multi-plunging airfoils.

The lift and drag coefficients for the upper and lower airfoils are also computed for Re $=150,300$ and 600 and the comparison between the $C_{l}$ and $C_{d}$ of the upper airfoil for these cases are presented in Figs. 8.9 and 12, respectively. It should be mentioned that the magnitude of the lower airfoil instantaneous lift coefficient is equal to that of the upper airfoil but mirrored.

Figure 8.9 shows upper airfoil's $C_{l}$ versus $\tau$ for $\mathrm{Re}=75,150,300$ and 600 . As shown, Re does not change lift coefficient noticeably and most of the changes occur between 0.25 $<\tau<0.5$, when the airfoils finish their first quarter stroke and start to move towards each 


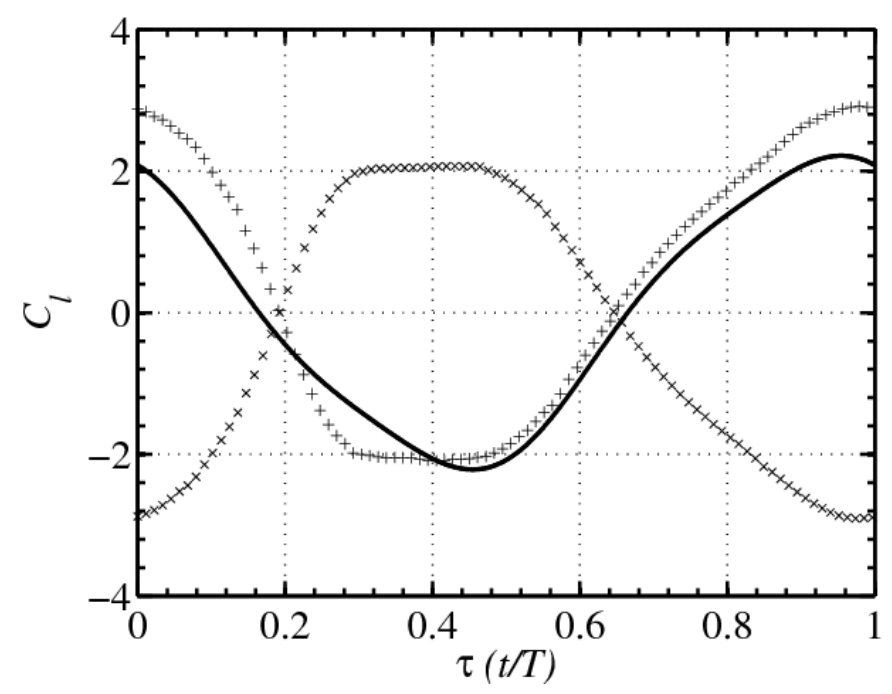

Figure 8.7: Instantaneous lift coefficient $\left(C_{l}\right)$ versus nondimensional time $(\tau)$ for $\operatorname{Re}=75$; $(+), C_{l}$ of the upper airfoil; $(\times), C_{l}$ of the lower airfoil; (-), $C_{l}$ of a single plunging airfoil.

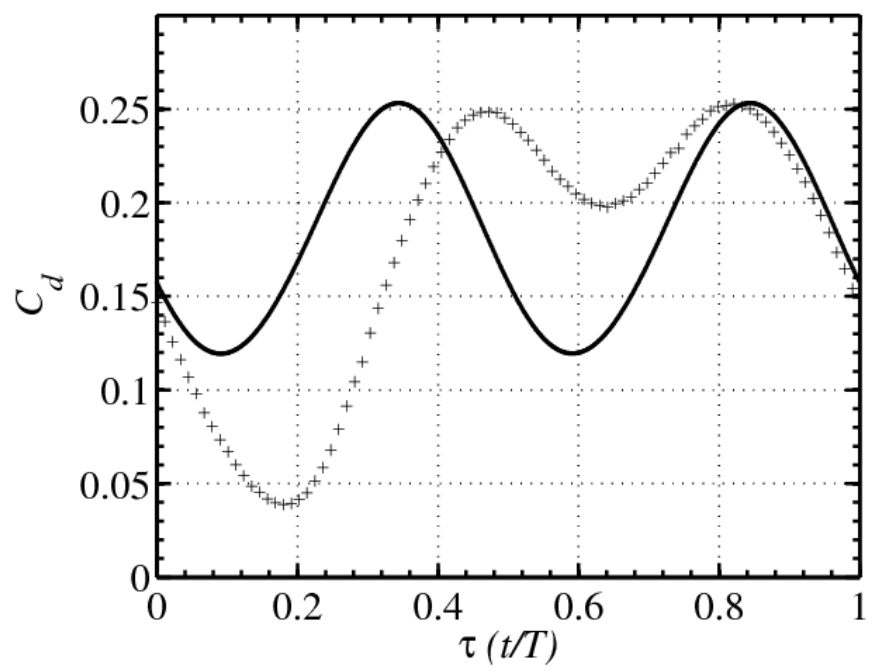

Figure 8.8: Instantaneous drag coefficient $\left(C_{d}\right)$ versus nondimensional time $(\tau)$ for $\operatorname{Re}=75$; $(+), C_{d}$ of the upper and lower airfoils; (-), $C_{d}$ of a single plunging airfoil.

other. It is also observed that the difference between the $C_{l}$ for $\operatorname{Re}=75$ and 150 is higher than the difference between $\mathrm{Re}=150$ and 300. These differences are even more noticeable than the difference between $\mathrm{Re}=300$ and 600 , which could be due to the lowered viscous forces when Re increases.

The effect of Re on the upper airfoil's $C_{d}$ is shown in Fig. 8.10, where Re is found to 


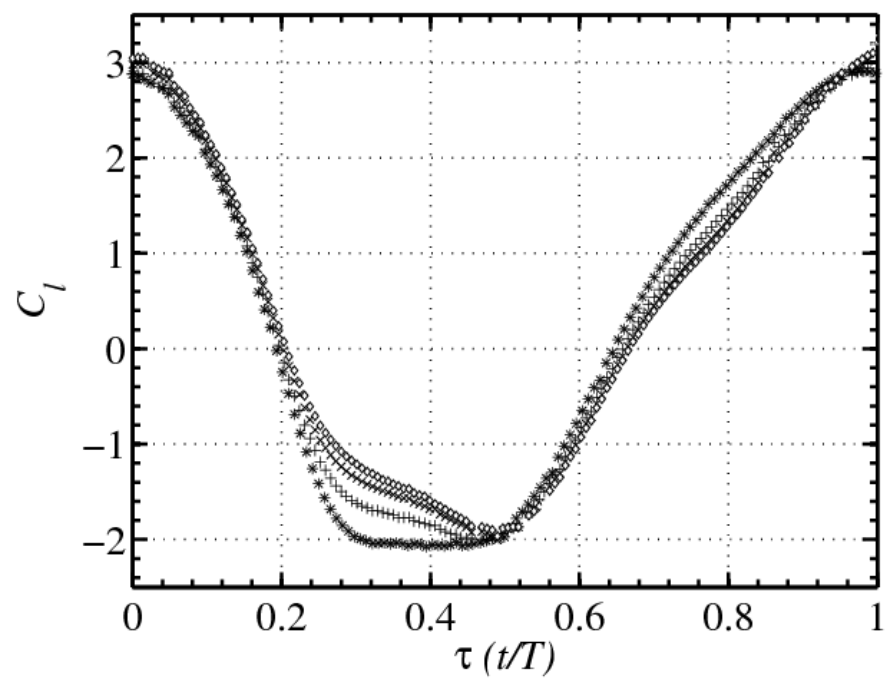

Figure 8.9: Instantaneous lift coefficient $\left(C_{l}\right)$ of the upper airfoil versus nondimensional time $\left.(\tau) ;{ }^{*}\right), \operatorname{Re}=75 ;(+), \operatorname{Re}=150 ;(\times), \operatorname{Re}=300 ;(\diamond), \operatorname{Re}=600$.

have more influence on the drag coefficients compared to those observed on lift coefficients, Fig. 8.9. There are two $C_{d_{\max }}$ for each period of plunging oscillations for all investigated Re. The first $C_{d_{\max }}$ is obtained at $\tau \approx 0.5$ and the second $C_{d_{\max }}$ is obtained at $\tau \approx 0.85$, where the airfoils are in the second half stroke. The two maximum drag coefficients are almost equal at $\operatorname{Re}=75$; however, increasing Re causes the first $C_{d_{\max }}$ to be smaller than the second one. There are also two minimum drag coefficients $\left(C_{d_{\text {min }}}\right)$; the first one is obtained at $\tau \approx 0.2$, where the airfoils move away from each other. Increasing Re, in this case, decreases $C_{d_{m i n}}$. The second $C_{d_{\min }}$ is obtained at $\tau \approx 0.6$ and decreases when Re increases. Moreover, the airfoil in Fig. 8.10 is in the so-called drag-producing regime at $\mathrm{Re}=75$, whereas increasing Re and the subsequent decrease in the first $C_{d_{\min }}$ causes the airfoil to produce thrust at the beginning of the stroke at the higher considered Reynolds numbers.

Figs. 8.11(a-d) show the corresponding flow structures when the first $C_{d_{\max }}$ is obtained for the investigated Re. The lighter color shows a higher pressure region. According to Fig. 8.10, the first $C_{d_{\max }}$ decreases (the second $C_{d_{\max }}$ follows the same trend) when Re increases and as can be seen in Figs. 8.11(a-d) the leading edge vortices (LEVs) of the upper and lower airfoils have the highest interaction at $\mathrm{Re}=75$, decreasing for the higher Re, 


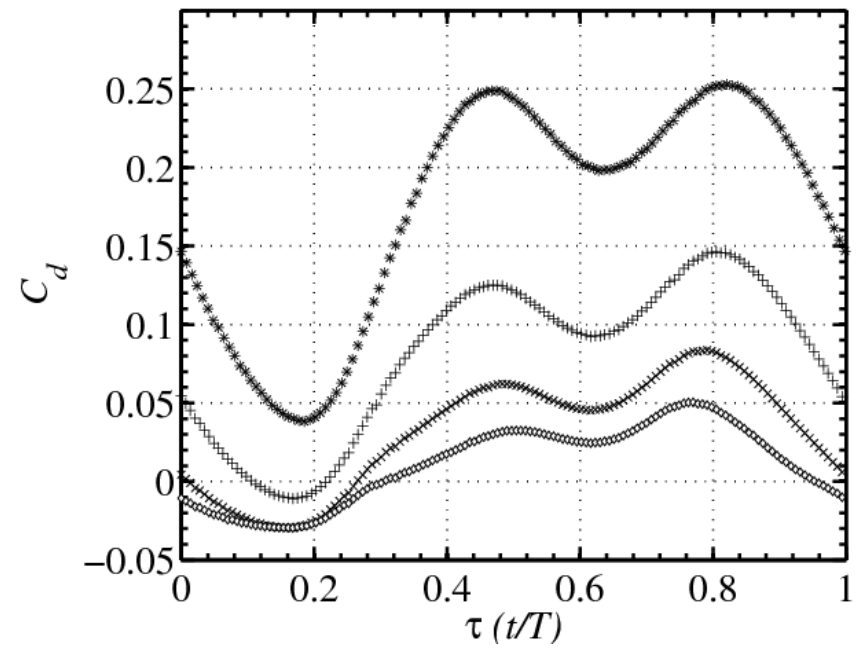

Figure 8.10: Instantaneous drag coefficient $\left(C_{d}\right)$ of the upper airfoil versus nondimensional time $(\tau) ;\left({ }^{*}\right), \operatorname{Re}=75 ;(+), \operatorname{Re}=150 ;(\times), \operatorname{Re}=300 ;(\diamond), \operatorname{Re}=600$.

which could explain the decreasing trend of the first $C_{d_{\max }}$.

There are also two minimum drag coefficients $\left(C_{d_{m i n}}\right)$; the first one is obtained at $\tau \approx 0.2$,

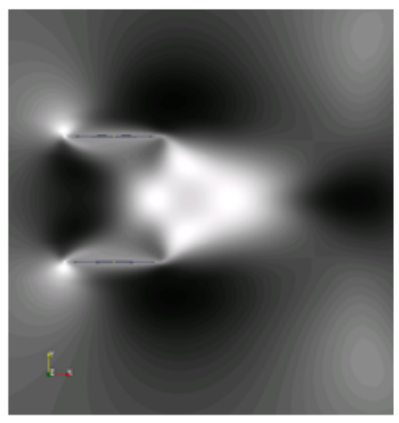

(a)

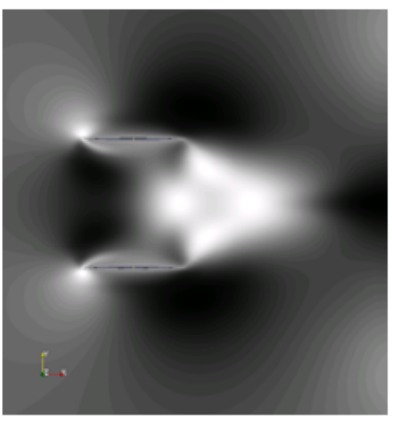

(b)

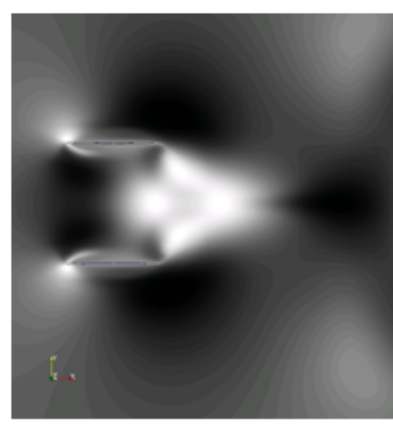

(c)

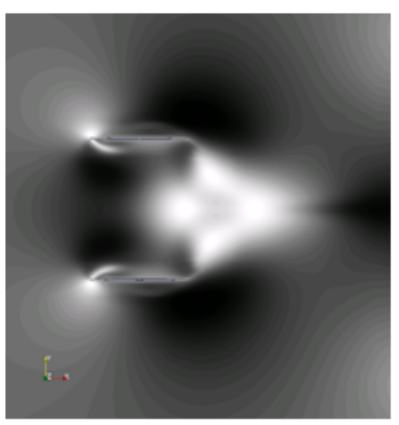

(d)

Figure 8.11: Vortical patterns around the airfoils when the first $C_{d_{\max }}$ is obtained; (a), Re $=75$; (b) $\operatorname{Re}=150 ;(\mathrm{c}), \operatorname{Re}=300 ;(\mathrm{d}), \operatorname{Re}=600$.

where the airfoils move away from each other. Increasing Re, in this case, decreases $C_{d_{\text {min }}}$. Figs. 8.12(a-d) show the flow structures when $C_{d_{m i n}}$ is obtained. Fig. 8.12(a) corresponds to Re $=75$ where high pressure flow surrounds the airfoils. As mentioned previously, a positive $C_{d_{\text {min }}}$ is obtained at $\operatorname{Re}=75$ at $\tau \approx 0.2$. Let's call the upper airfoil top surface the suction side, where the same terminology is used for the lower airfoil down surface. As observed, by increasing $\operatorname{Re}$ from $\mathrm{Re}=75$ to 150 , lower pressure is exerted on the suction surface and 
a recirculation region is formed, leading to the lowered $C_{d_{\min }}$ at $\operatorname{Re}=150\left(C_{d_{\min }}<0\right.$ or thrust). The strength of the so-called recirculation region (low pressure area) increases for the higher Reynolds numbers and as a result, the airfoils generates more thrust at the higher investigated Re, Figs. 8.12(b-d).

The mean $C_{l}$ and $C_{d}\left(\bar{C}_{l}\right.$ and $\left.\bar{C}_{d}\right)$ for the considered Re are presented in Table 8.2. The

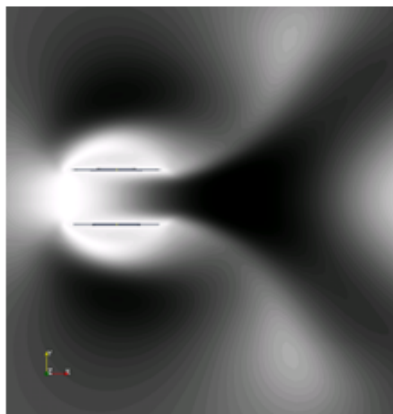

(a)

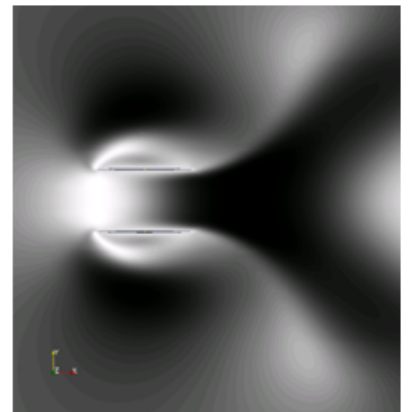

(b)

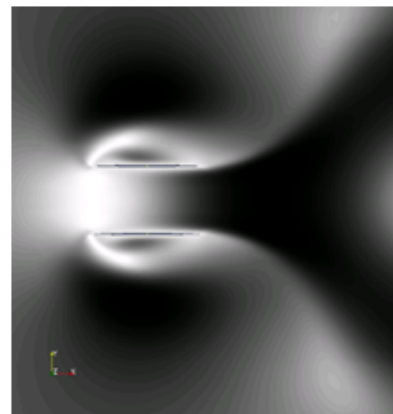

(c)

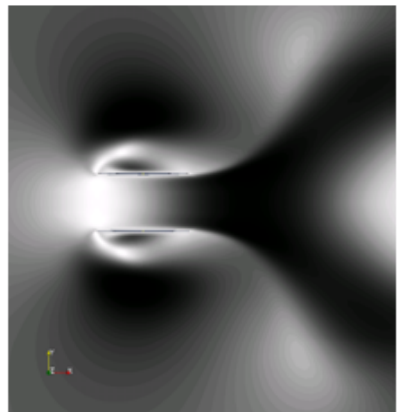

(d)

Figure 8.12: Vortical patterns around the airfoils when the first $C_{d_{\min }}$ is obtained; (a), $\operatorname{Re}=$ 75; (b) $\operatorname{Re}=150 ;(\mathrm{c}), \operatorname{Re}=300 ;(\mathrm{d}), \operatorname{Re}=600$.

values are presented for each half cycle as well as the whole cycle of the plunging motion. It can be seen that increasing Re slightly increases $\bar{C}_{l}$ and decreases $\bar{C}_{d}$ more noticeably in a cycle, resulting in a higher aerodynamic performance $\left(\frac{\overline{C_{l}}}{\bar{C}_{d}}\right)$.

Table 8.2: Mean lift and drag coefficients $\left(\bar{C}_{l}\right.$ and $\left.\bar{C}_{d}\right)$ for the considered Re.

\begin{tabular}{cccc}
\hline \hline Re & First half $(0 \leq \tau \leq 0.5)$ & Second half $(0.5 \leq \tau \leq 1)$ & Whole cycle $(0 \leq \tau \leq 1)$ \\
\hline & & & \\
75 & $\bar{C}_{l}=-0.359, \bar{C}_{d}=0.129$ & $\bar{C}_{l}=0.979, \bar{C}_{d}=0.218$ & $\bar{C}_{l}=0.313, \bar{C}_{d}=0.174$ \\
150 & $\bar{C}_{l}=-0.2, \bar{C}_{d}=0.049$ & $\bar{C}_{l}=0.82, \bar{C}_{d}=0.111$ & $\bar{C}_{l}=0.32, \bar{C}_{d}=0.079$ \\
300 & $\bar{C}_{l}=-0.05, \bar{C}_{d}=0.008$ & $\bar{C}_{l}=0.765, \bar{C}_{d}=0.055$ & $\bar{C}_{l}=0.361, \bar{C}_{d}=0.032$ \\
600 & $\bar{C}_{l}=-0.039, \bar{C}_{d}=-0.006$ & $\bar{C}_{l}=0.734, \bar{C}_{d}=0.028$ & $\bar{C}_{l}=0.389, \bar{C}_{d}=0.011$ \\
\hline
\end{tabular}

\subsubsection{Effect of the frequency of oscillations}

The flow field of the upper and lower airfoils is then simulated for various frequencies of oscillations and parameter settings, listed in Table 8.1. The Strouhal number corresponding to the investigated frequencies of oscillations is calculated where $L_{r e f}=Y_{0}$ and $U_{r e f}=U_{\infty}$. 
According to the results presented in this section, it is found that there is an optimum frequency of oscillations, resulting in the highest thrust generation between the investigated frequencies. Drag coefficient for this frequency $(f=0.5 \mathrm{~Hz}, \mathrm{St}=1.571)$ has the lowest $C_{d_{\max }}$ and the best thrust generation both in terms of the magnitude and the time interval during which thrust is generated. Frequency also has very significant effects on $C_{l}$; however, the influence is mostly quantitative, increasing the peak values.

The $C_{l}$ and $C_{d}$ variations for $f=0.5 \mathrm{~Hz}(\mathrm{St}=1.571), f=1 \mathrm{~Hz}(\mathrm{St}=3.142)$ and $f=2$ $\mathrm{Hz}(\mathrm{St}=6.283)$ are calculated. As seen in Fig. 8.13, the magnitude of $C_{l_{\max }}$, in this case, is higher than the magnitude of the minimum lift coefficient $\left(C_{l_{\min }}\right)$ at $f=0.25(\mathrm{St}=0.785)$; $C_{l_{\max }} \approx 3$ and $C_{l_{\min }} \approx 2$. Increasing the frequency of oscillations from $f=0.25(\mathrm{St}=0.785)$ to $f=0.5(\mathrm{St}=1.571)$ increases the magnitude of both $C_{l_{\max }}$ and $C_{l_{\min }}$ and changes them to 7.5 and 6 , respectively. Further increase of $f$ from $f=0.5$ to $f=1$ also increases the magnitude of the lift extreme values; however, $\left|C_{l_{\min }}\right| \approx 15$, which is higher than $\left|C_{l_{\max }}\right| \approx$ 9.5. This trend is also repeated for $f=2$, where $\left|C_{l_{\max }}\right| \approx 11$ and $\left|C_{l_{\min }}\right| \approx 16$.

The effect of the frequency of oscillations $(f)$ on $C_{d}$ is also investigated and presented

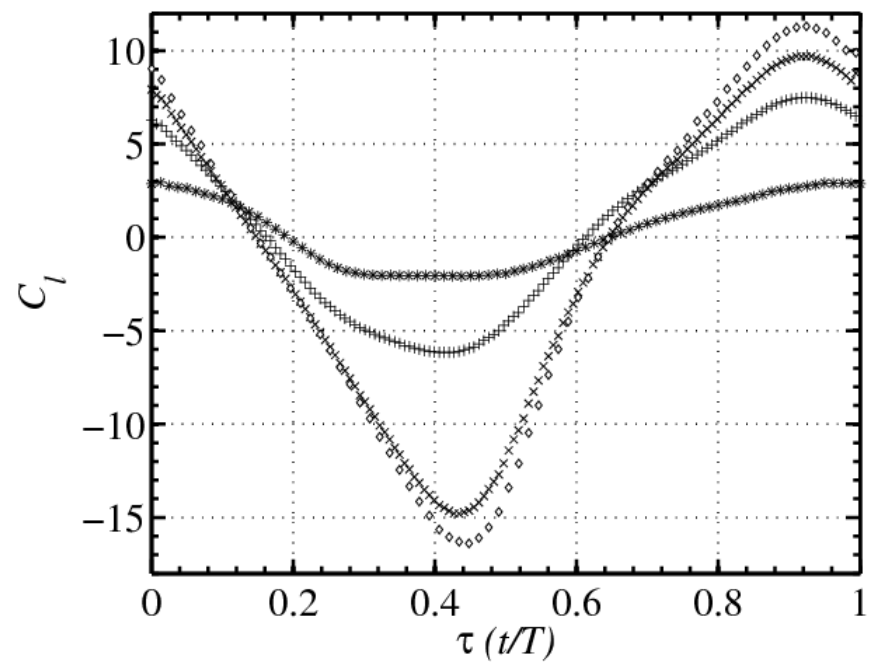

Figure 8.13: Instantaneous lift coefficient $\left(C_{l}\right)$ versus nondimensional time $(\tau)$ for the upper airfoil: $\left.{ }^{*}\right) f=0.25,(+) f=0.5,(\times) f=1,(\diamond) f=2$.

in Fig. 8.14. As can be seen, for $f=0.25 C_{d}$ has two maximums $\left(C_{d_{\max }}\right)$ at $\tau \approx 0.4$ and 
$\tau \approx 0.8$. It means that both of the extremum values are obtained when the airfoils move during the second quarter period of each half stroke for which the airfoil-airfoil interaction is more significant than the first stroke. Interestingly, increasing $f$ from 0.25 to 0.5 reduces the number of $C_{d_{\max }}$ from two to one, Fig. 8.14. Moreover, increasing $f$ lowers the magnitude of $C_{d_{\max }}$ from $C_{d_{\max }} \approx 0.25$ at $f=0.25$ to $C_{d_{\max }} \approx 0.15$ at $f=0.5$. Moreover, the airfoil only produces drag at $f=0.25$, whereas it produces both drag and thrust at $f=0.5$ (Fig. 8.14). Thrust coefficient is namely a negative drag coefficient $\left(C_{t}=-C_{d}\right)$. Exploring Fig. 8.14 at $f=0.5$ shows that the airfoil produces thrust (i.e., thrust-producing regime) for $0<\tau<$ 0.5 with the maximum thrust coefficient $\left(C_{t_{\max }}\right)$ of 0.25 at $\tau \approx 0.05$ at the beginning of the plunging cycle. Then, it produces drag for $0.5<\tau<0.8$ with $C_{d_{\max }} \approx 0.15$ at $\tau \approx 0.65$, where the airfoils move towards each other, and finally produces thrust for $0.8<\tau<1$. Increasing $f$ from 0.5 to 1 causes the airfoil to produce more drag and less thrust compared to what observed for $f=0.5$. That is, the airfoil produces thrust for $0<\tau<0.4$ with $C_{t_{\max }}$ $\approx 0.225$ at $\tau \approx 0.2$ and produces drag for $0.4<\tau<1$ with $C_{d_{\max }} \approx 0.425$ at $\tau \approx 0.55$. Hence the maximum thrust coefficient is decreased and obtained with lag, and the maximum drag coefficient is increased and obtained with a lead when frequency of oscillations increases from $f=0.5$ to 1 . Increasing $f$ from 1 to 2 does change the time interval in which drag and thrust are produced; however, the magnitude of the maximum drag and thrust coefficients are both decreased.

As mentioned in the previous paragraph, the airfoils generate thrust for some of the investigated frequencies. This is more investigated by exploring the flow structures for the instant at which the minimum drag coefficient $\left(C_{d_{\min }}\right)$ is obtained. Figs. 8.15(a-d) show these flow structures. As can be seen the flow field of $f=0.25$ (drag producing) is different from $f=0.5,1$ and 2 (thrust producing). As seen, by increasing $f=0.25$ (Fig. 8.15(a)) to $f$ $=0.5($ Fig. 8.15(b)), the bifurcated downstream wake at $f=0.25$ is converted into separate trailing edge vortices (TEVs) at $f=0.5$. The high pressure region around the airfoils at $f$ $=0.25$ is also confined to the LE. These changes are accompanied by the decreased $C_{d_{\min }}$ 


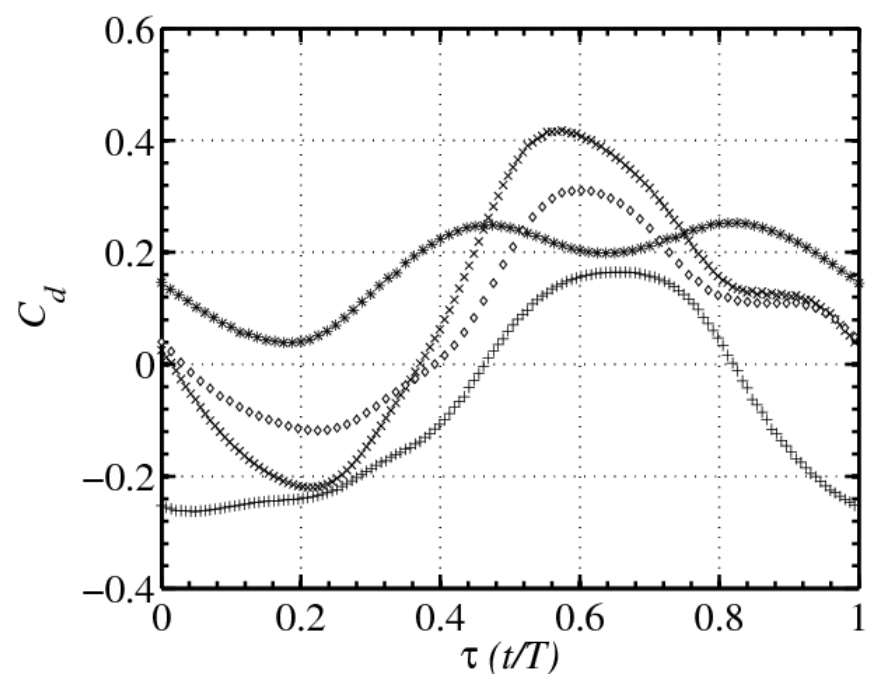

Figure 8.14: Instantaneous drag coefficient $\left(C_{d}\right)$ versus nondimensional time $(\tau)$ for the upper airfoil: $(*) f=0.25,(+) f=0.5,(\times) f=1,(\diamond) f=2$.

at $f=0.5$ compared to $f=0.25$. As explained before, the magnitude of $C_{d_{\min }}\left(C_{d_{\min }}<\right.$ 0 , thrust) is slightly lower at $f=1$ compared to that of $f=0.5$. The flow structure for $f=0.5$ and 1 are very similar except that the high pressure region at $f=0.5$ is closer to each airfoil's surface than that of $f=1$. Moreover, the extent of the higher pressure flow field between the airfoils at $f=0.5$ is more than that at $f=1$. Comparing Figs. 8.15(c, d) shows that the flow structures are very similar and the main difference exists around the LE of both airfoils.

Table 8.3 shows $\bar{C}_{l}$ and $\bar{C}_{d}$ for each half stroke as well as the whole plunging cycle for

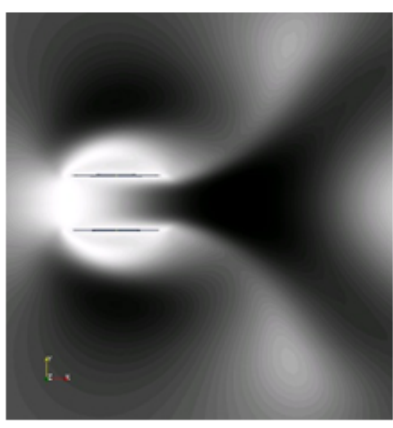

(a)

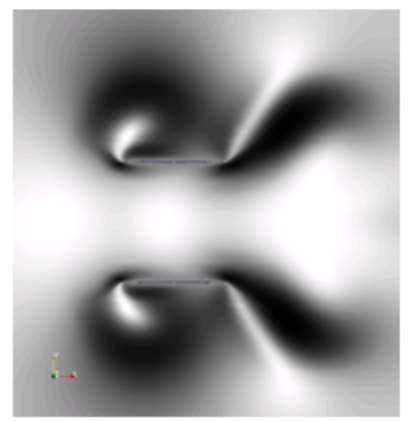

(b)

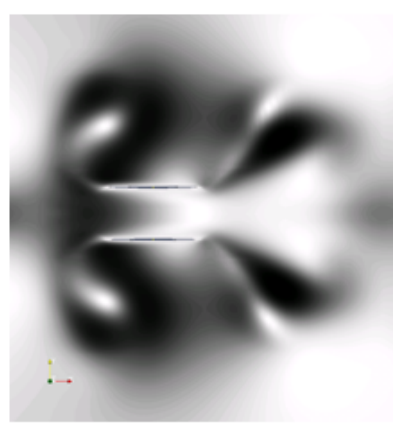

(c)

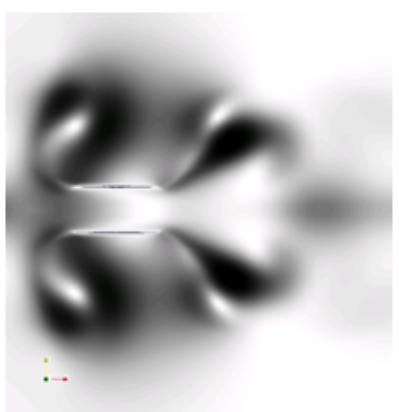

(d)

Figure 8.15: Vortical patterns around the airfoils when the first $C_{d_{\min }}$ is obtained; (a), $f=$ 0.25 ; (b), $f=0.5 ;(\mathrm{c}), f=1 ;(\mathrm{d}), f=2$. 
the considered frequencies, where there exist downward mean lift $\left(\bar{C}_{l}<0\right)$ and mean thrust $\left(\bar{C}_{d}<0\right)$ for some of the frequencies of oscillations.

Table 8.3: Mean lift and drag coefficients $\left(\bar{C}_{l}\right.$ and $\left.\bar{C}_{d}\right)$ for the considered frequencies of oscillations $(f)$.

\begin{tabular}{cccc}
\hline \hline$f$ & First half $(0 \leq \tau \leq 0.5)$ & Second half $(0.5 \leq \tau \leq 1)$ & Whole cycle $(0 \leq \tau \leq 1)$ \\
\hline 0.25 & $\bar{C}_{l}=-0.359, \bar{C}_{d}=0.129$ & $\bar{C}_{l}=0.979, \bar{C}_{d}=0.218$ & $\bar{C}_{l}=0.313, \bar{C}_{d}=0.174$ \\
0.5 & $\bar{C}_{l}=-1.975, \bar{C}_{d}=-0.181$ & $\bar{C}_{l}=3.254, \bar{C}_{d}=0.026$ & $\bar{C}_{l}=0.674, \bar{C}_{d}=-0.078$ \\
1 & $\bar{C}_{l}=-5.226, \bar{C}_{d}=-0.057$ & $\bar{C}_{l}=3.102, \bar{C}_{d}=0.246$ & $\bar{C}_{l}=-1.083, \bar{C}_{d}=0.094$ \\
2 & $\bar{C}_{l}=-5.656, \bar{C}_{d}=-0.034$ & $\bar{C}_{l}=3.334, \bar{C}_{d}=0.188$ & $\bar{C}_{l}=-1.161, \bar{C}_{d}=0.077$ \\
\hline
\end{tabular}

\subsubsection{Effect of the amplitude of oscillations}

The effect of the amplitude of plunging oscillations, $Y_{0}$ in Equations 8.1, is investigated for the values listed in Table 8.1. It is found, based on the following results, that increasing the amplitude $\left(Y_{0}\right)$ when $g=1.5 c$ and $3 c$ decreases the mean drag coefficient, although $C_{l}$ increases when $Y_{0}$ increases. Hence, $\frac{\bar{C}_{l}}{\bar{C}_{d}}$ increases at the higher amplitudes of oscillations.

As can be seen in Fig. 8.16, increasing the amplitude of oscillations from $Y_{0}=0.3 c$ (St $=0.471)$ to $0.5 c(\mathrm{St}=0.785)$ at $g=1.5 \mathrm{c}$ increases the magnitude of $C_{l_{\max }}$ from 1.9 to $3(\approx$ $58 \%$ of increase) and increases the magnitude of $C_{l_{\min }}$ from 1.5 to $2(\approx 33 \%$ of increase). Therefore, $Y_{0}$ affects the maximum lift coefficient more than the minimum value.

Figs. 8.17(a, b) show the flow field around the airfoils when $C_{l_{\min }}$ is obtained for $Y_{0}=$ $0.3 c$ and $0.5 c$. As expected, the higher amplitude of oscillations results in more airfoil-airfoil interaction. The interaction is observed around the leading/trailing edge of the airfoils, which is strengthened for $Y_{0}=0.5 c$ compared to that of $Y_{0}=0.3 c$. As a result, the magnitude of $C_{l_{\min }}$ decreases with $Y_{0}$. Figs. 8.17(c, d) correspond to the $C_{l_{\max }}$ in Fig. 8.16. As mentioned before, $C_{l_{\max }}$ increases at the higher $Y_{0}$. Fig. $8.17(\mathrm{~d})$ shows that a higher pressure region exists at the LE of both airfoils at $Y_{0}=0.5 \mathrm{c}$, compared to $Y_{0}=0.3 c$. Moreover, the downstream is deflected upward (for the upper airfoil) and downward (for the lower one) at $Y_{0}=0.5 c$, affecting the flow structure at the airfoils' TE. These complex vortical patterns 


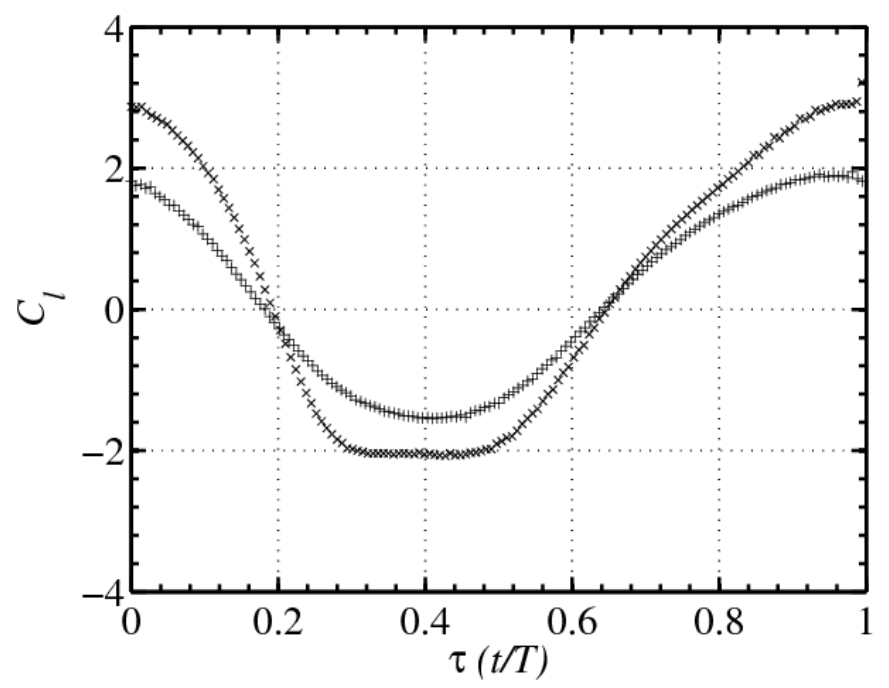

Figure 8.16: Instantaneous lift coefficient $\left(C_{l}\right)$ versus nondimensional time $(\tau)$ for the upper airfoil: $(+) Y_{0}=0.3 c,(\times) Y_{0}=0.5 c$.

and trends at the higher amplitude explain the increase of $C_{l_{\max }}$ in Fig. 8.16.

Figure 8.18 shows $C_{d}$ versus $\tau$ for $Y_{0}=0.3 c$ and $0.5 c(g=1.5 c)$. There are two $C_{d_{\max }}$

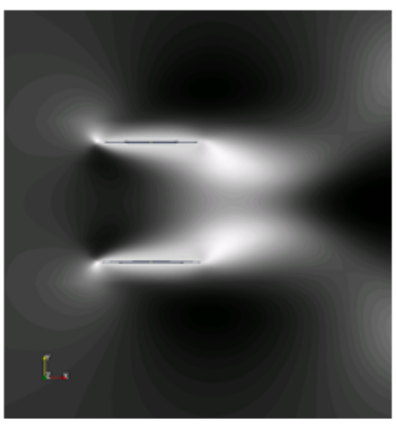

(a)

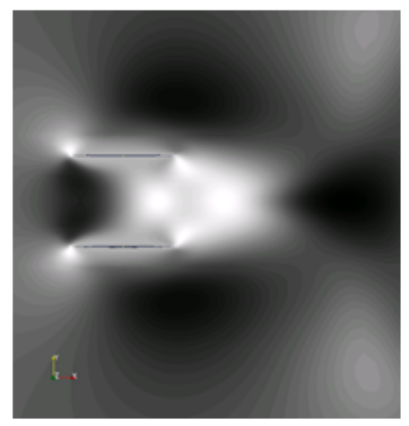

(b)

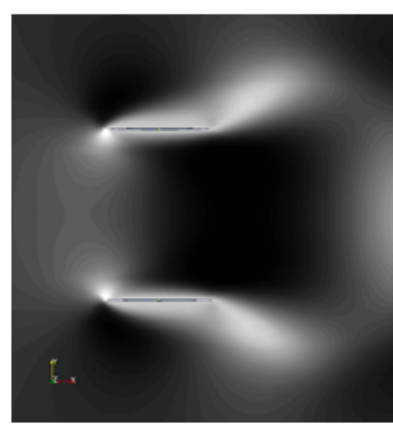

(c)

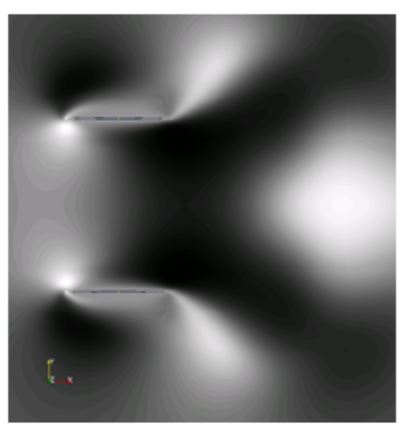

(d)

Figure 8.17: Flow field around the airfoils; (a), $C_{l_{\min }}$ at $Y_{0}=0.3 c$; (b), $C_{l_{\min }}$ at $Y_{0}=0.5 c$; (c), $C_{l_{\max }}$ at $Y_{0}=0.3 c ;(\mathrm{d}), C_{l_{\max }}$ at $Y_{0}=0.5 c$.

values for $Y_{0}=0.3 c$, where $C_{d_{\max }} \approx 0.34$ at $\tau \approx 0.4$ and $C_{d_{\max }} \approx 0.36$ at $\tau \approx 0.85$. Increasing $Y_{0}$ from $Y_{0}=0.3 c$ to $0.5 c$ decreases both of the maximum values and changes them to $C_{d_{\max }}$ $\approx 0.25$ at $\tau \approx 0.5$ (the first maximum) and $C_{d_{\max }} \approx 0.25$ at $\tau \approx 0.85$. Therefore, increasing $Y_{0}$ also induces a lag in the first $C_{d_{\max }}$. In other words, the mean drag coefficient decreases and the amplitude of $C_{d}$ (the difference between the maximum and minimum $C_{d}$ ) increases when $Y_{0}$ increases. 
Figs. $8.19(\mathrm{a}, \mathrm{b})$ show the flow structure when the first $C_{d_{\min }}$ is obtained. Increasing

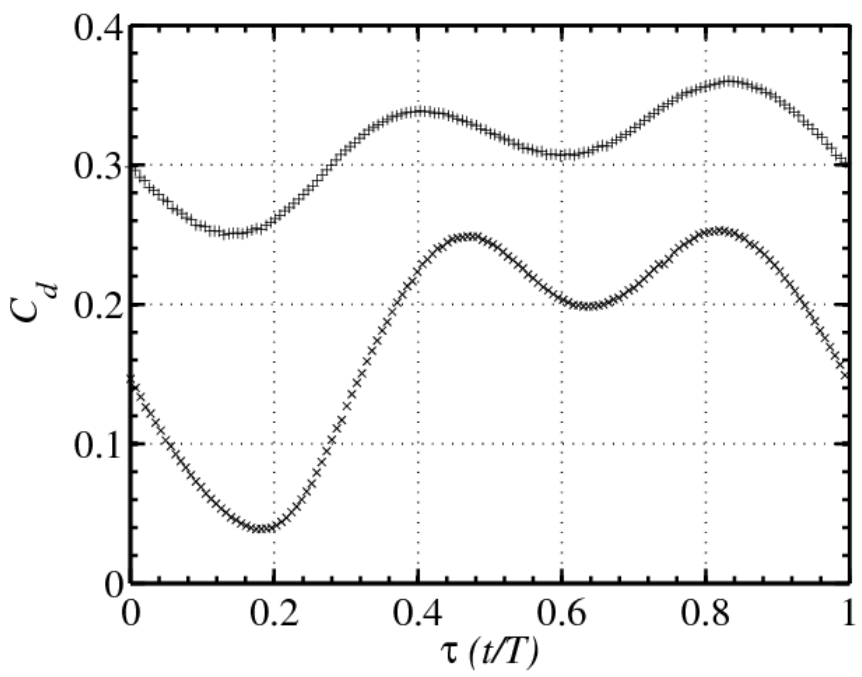

Figure 8.18: Instantaneous drag coefficient $\left(C_{d}\right)$ versus nondimensional time $(\tau)$ for the upper airfoil: $(+) Y_{0}=0.3 c,(\times) Y_{0}=0.5 c$.

$Y_{0}$ from $Y_{0}=0.3 c$ to $0.5 c$ generates higher pressure region around the airfoils, where the interaction between the upper and lower airfoils' high pressure regions is more significant at $Y_{0}=0.5 c$ compared to that of $Y_{0}=0.3 c$. The downstream flow is also bifurcated at $Y_{0}=$ $0.5 c$, which is not observed at $Y_{0}=0.3 c$. As mentioned earlier, both of the $C_{d_{\max }}$ decrease at the higher considered amplitude $\left(Y_{0}=0.5 c\right)$. The comparison between the corresponding flow patterns of $Y_{0}=0.3 c$ and $0.5 c$ is presented in Figs. $8.19(\mathrm{c}, \mathrm{d})$, when the second $C_{d_{\max }}$ is obtained.

Table 8.4 shows $\bar{C}_{l}$ and $\bar{C}_{d}$ for the considered amplitudes of oscillations when $g=1.5 c$. It is seen that $\bar{C}_{l}$ and $\bar{C}_{d}$ increases and decreases, respectively, at the higher $Y_{0}$, resulting in a higher $\frac{\bar{C}_{l}}{\bar{C}_{d}}$.

Figure 8.20 shows the effect of $Y_{0}$ on $C l$ versus $\tau$, when $g=3 c$. As expected the lift coefficients show a more harmonic pattern when compared to those obtained at $g=$ $1.5 c$ (Fig. 8.16). This is mainly due to the lower airfoil-airfoil interaction when $g=3 c$. Consequently, the magnitude of the $C_{l_{\max }}$ and $C_{l_{\min }}$ are closer than those obtained at $g$ $=1.5 c$, Fig. 8.16. However, the airfoil-airfoil interaction still produces different extremum 


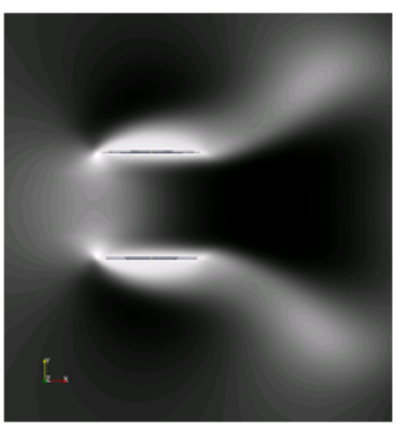

(a)

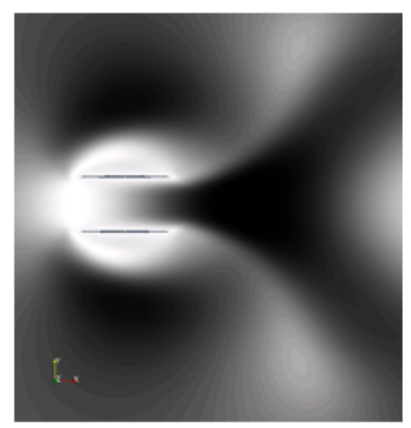

(b)

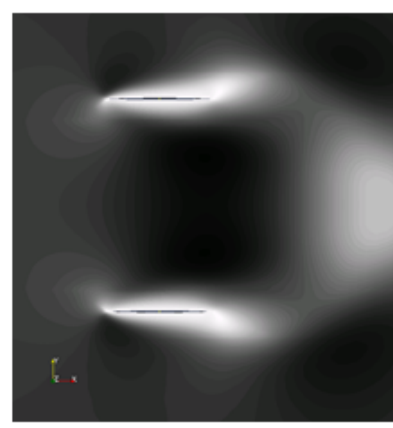

(c)

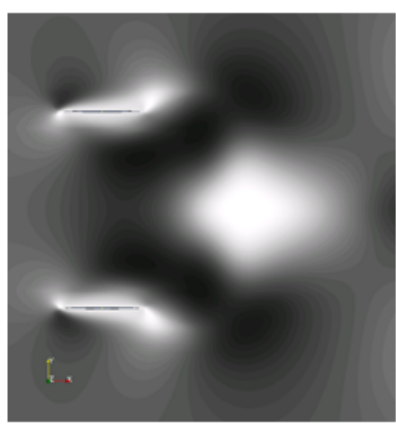

(d)

Figure 8.19: Flow field around the airfoils; (a), $C_{d_{\text {min }}}$ at $Y_{0}=0.3 c$; (b), $C_{d_{\text {min }}}$ at $Y_{0}=0.5 c$; (c), second $C_{d_{\max }}$ at $Y_{0}=0.3 c ;(\mathrm{d})$, second $C_{d_{\max }}$ at $Y_{0}=0.5 c$.

Table 8.4: Mean lift and drag coefficients $\left(\bar{C}_{l}\right.$ and $\left.\bar{C}_{d}\right)$ for the considered amplitude of oscillations $\left(Y_{0}\right)$ when $g=1.5 c$.

\begin{tabular}{cccc}
\hline \hline$Y_{0}$ & First half $(0 \leq \tau \leq 0.5)$ & Second half $(0.5 \leq \tau \leq 1)$ & Whole cycle $(0 \leq \tau \leq 1)$ \\
\hline $0.3 c$ & $\bar{C}_{l}=-0.342, \bar{C}_{d}=0.295$ & $\bar{C}_{l}=0.725, \bar{C}_{d}=0.329$ & $\bar{C}_{l}=0.186, \bar{C}_{d}=0.312$ \\
$0.5 c$ & $\bar{C}_{l}=-0.359, \bar{C}_{d}=0.129$ & $\bar{C}_{l}=0.979, \bar{C}_{d}=0.218$ & $\bar{C}_{l}=0.313, \bar{C}_{d}=0.174$ \\
\hline
\end{tabular}

values. The magnitude of $C_{l_{\max }}$ and $C_{l_{\min }}$ are 2.3 and 2.2 for $Y_{0}=0.5 c(\mathrm{St}=0.785), 2.8$ and 2.6 for $Y_{0}=0.7 c(\mathrm{St}=1.1)$ and 3.3 and 2.8 for $Y_{0}=0.9 c(\mathrm{St}=1.414)$.

Figure 8.21 shows $C_{d}$ versus $\tau$ when $g=3 c$. In each period of oscillation $C_{d}$ has two

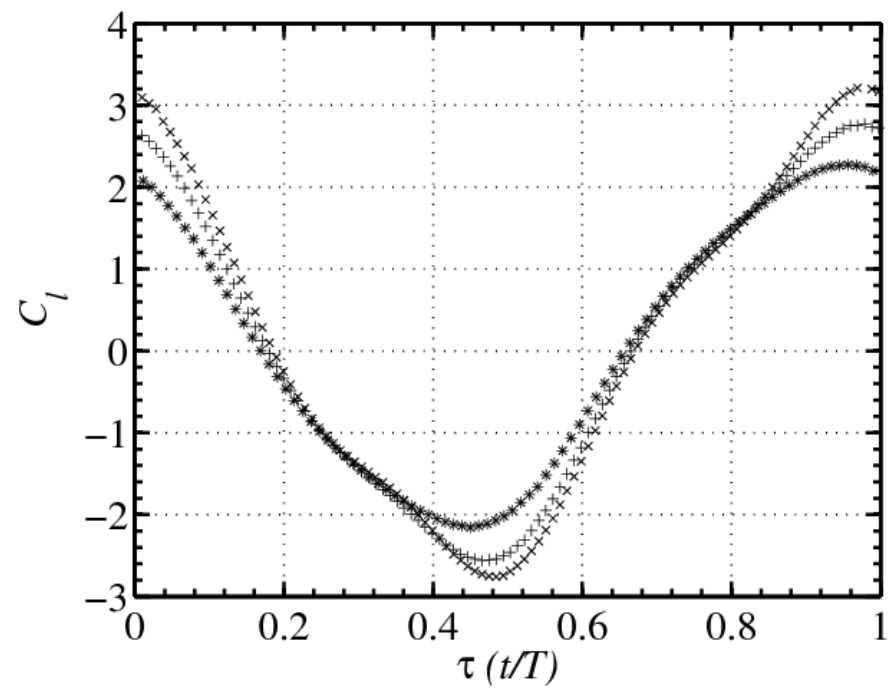

Figure 8.20: Instantaneous lift coefficient $\left(C_{l}\right)$ versus nondimensional time $(\tau)$ for the upper airfoil at $g=3 c:(*) Y_{0}=0.5 c,(+) Y_{0}=0.7 c,(\times) Y_{0}=0.9 c$. 
maximum and two minimum values for all the considered plunging amplitudes. Increasing the amplitude of oscillations from $Y_{0}=0.5 c$ to $0.7 c$ decreases the magnitude of the first $C_{d_{\max }}$ from $C_{d_{\max }}=0.3$ at $\tau \approx 0.36$ to $C_{d_{\max }}=0.22$ at $\tau \approx 0.39$. The second $C_{d_{\max }}$ and the minimum drag coefficient also undergo similar changes versus $Y_{0}$. The maximum and minimum drag coefficients decrease for further increase of $Y_{0}$ from $Y_{0}=0.7 c$ to $0.9 c$ such that the airfoil produces thrust at $Y_{0}=0.9 \mathrm{c}$ between $0.05<\tau<0.25$.

Table 8.5 presents $\bar{C}_{l}$ and $\bar{C}_{d}$ for the considered amplitudes of oscillations when $g=3 c$,

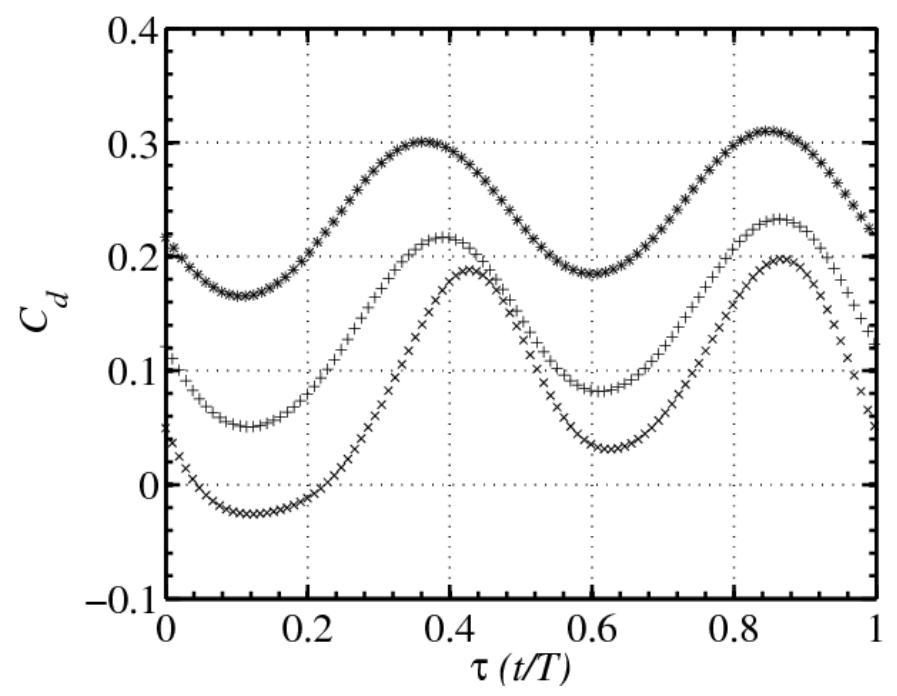

Figure 8.21: Instantaneous drag coefficient $\left(C_{d}\right)$ versus nondimensional time $(\tau)$ for the upper airfoil at $g=3 c:\left({ }^{*}\right) Y_{0}=0.5 c,(+) Y_{0}=0.7 c,(\times) Y_{0}=0.9 c$.

and it shows that increasing $Y_{0}$ has a similar increasing effect on $\frac{\bar{C}_{l}}{\bar{C}_{d}}$ to the one at $g=1.5 c$.

Table 8.5: Mean lift and drag coefficients $\left(\bar{C}_{l}\right.$ and $\left.\bar{C}_{d}\right)$ for the considered amplitude of oscillations $\left(Y_{0}\right)$ when $g=1.5 c$.

\begin{tabular}{cccc}
\hline \hline$Y_{0}$ & First half $(0 \leq \tau \leq 0.5)$ & Second half $(0.5 \leq \tau \leq 1)$ & Whole cycle $(0 \leq \tau \leq 1)$ \\
\hline $0.5 c$ & $\bar{C}_{l}=-0.565, C_{d}=0.233$ & $\bar{C}_{l}=0.704, C_{d}=0.245$ & $\bar{C}_{l}=0.075, C_{d}=0.239$ \\
$0.7 c$ & $\bar{C}_{l}=-0.52, \bar{C}_{d}=0.13$ & $\bar{C}_{l}=0.683, \bar{C}_{d}=0.153$ & $\bar{C}_{l}=0.088, \bar{C}_{d}=0.142$ \\
$0.9 c$ & $\bar{C}_{l}=-0.389, \bar{C}_{d}=0.059$ & $\bar{C}_{l}=0.679, \bar{C}_{d}=0.106$ & $\bar{C}_{l}=0.152, \bar{C}_{d}=0.083$ \\
\hline
\end{tabular}




\subsubsection{Effect of the airfoil spacing}

The effect of the airfoil spacing $(g)$ on the lift and drag coefficients is shown in Figs.!8.22 and 8.23, respectively, for the values listed in Table 8.1. Fig. 8.22 shows the lift coefficient versus $\tau$. It is observed from the figure that increasing $g$ affects $C_{l_{\max }}$ noticeably. The $C_{l_{\max }}$ decreases by increasing the spacing, which is due to the lowered airfoil-airfoil interaction influence. However, the magnitude of $C_{l_{m i n}}$ is not changed as it is obtained when the airfoils move away. Fig. 8.23 shows $C_{d}$ versus $\tau$, where both of the $C_{d_{\max }}$ values increase by increasing $g$. The single minimum drag coefficient at $g=1.5 c$ is also increased at $g=3 c$, where there are two maximum drag coefficients.

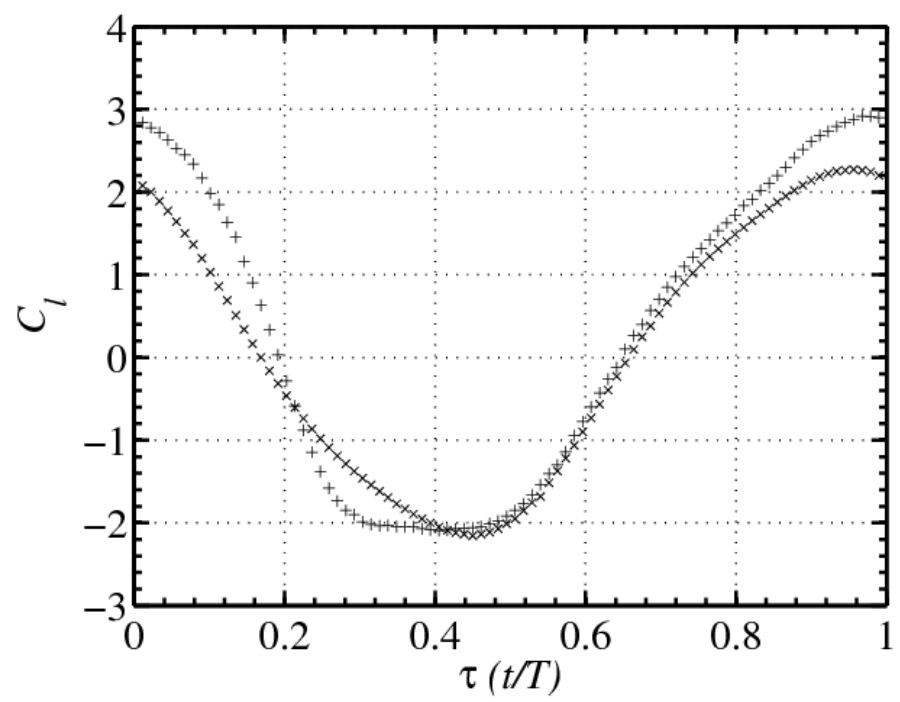

Figure 8.22: Instantaneous lift coefficient $\left(C_{l}\right)$ versus nondimensional time $(\tau)$ for the upper airfoil: $(+) g=1.5 c,(\times) g=3 c$.

\subsection{Summary and conclusion}

Simulations of a pair of multi-plunging ellipsoidal airfoils in low Reynolds number regime are conducted using two dimensional Navier-Stokes equations with a Finite Volume Method. 


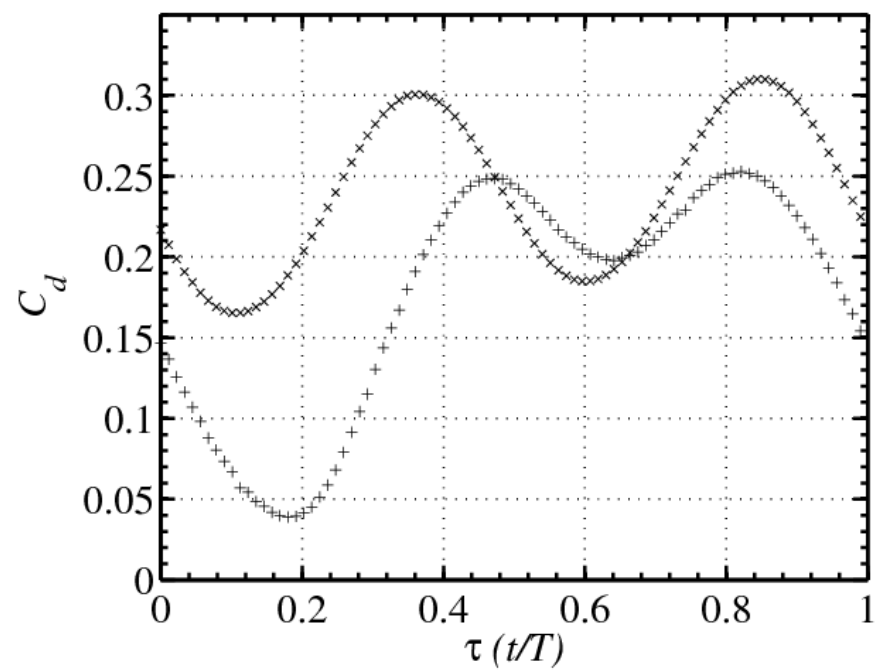

Figure 8.23: Instantaneous drag coefficient $\left(C_{d}\right)$ versus nondimensional time $(\tau)$ for the upper airfoil: $(+) g=1.5 c,(\times) g=3 c$.

A parametric study is performed and the effects of Reynolds number, frequency and amplitude of plunging oscillations $\left(f, Y_{0}\right)$ and the airfoil-airfoil spacing $(g)$ on the lift and drag coefficients are investigated. It is observed that the airfoil-airfoil interaction increases the maximum lift coefficient compared to a single plunging airfoil, while the minimum lift coefficient does not change noticeably compared to the similar single plunging airfoil. The observed quasi-sinusoidal pattern of the single airfoil's instantaneous lift coefficient exists in the multi-plunging airfoil simulation. It is shown that Reynolds number has fewer effects on the lift and drag coefficients than those of the other investigated parameters. Reynolds number alters the lift coefficients mostly during the plunging first half stroke, where the airfoils move away from each other. Unlikely, it affects drag coefficients more significantly than lift coefficients. The airfoil produces drag in the whole plunging cycle at the lower considered Reynolds numbers, whereas increasing Re causes the airfoil to generate thrust in some portions of the plunging cycle, at least for the Re range studied. Frequency of oscillations is shown to have major influence on the fluid forces, and changes both lift and drag coefficients noticeably. The extent of the thrust-producing region increases at the intermediate considered frequencies, but decreases at the higher ones. Therefore, there is an optimum frequency 
for having the highest possible thrust coefficient. The amplitude of oscillations is also another important factor to the peaks of lift and drag coefficients. As expected, the higher amplitude of oscillations means more airfoil-airfoil interaction, which consequently changes the peaks of lift and drag coefficient accordingly. The result of the presented two dimensional analysis is important when compared to the corresponding two dimensional study of a single plunging airfoil, revealing the importance of the vortical interaction effects. The findings of this study and the similar studies will also provide a basis for a better understanding of the respective three dimensional applications and their flow structures. In the next chapter, multi figure-of-eight flapping airfoils are studied. 


\section{Chapter 9}

\section{Multi figure-of-eight flapping airfoils}

The objective of the present chapter is to investigate the low Reynolds number (LRN) fluid dynamics of two airfoils in figure-of-eight-like flapping motion. A library is developed to handle multi-body problems and is utilized to simulate the simultaneous motion of a pair of airfoils, and a few test cases are investigated. Moreover, a brief parametric study is performed to explore how the pitching amplitude of oscillations affects the lift and drag signatures. The results show that the aerodynamic performance (lift and drag coefficients) of the simulated airfoils is lower than the respective single flapping airfoil. This raises the question of how a three-dimensional rotating-flapping configuration using the same investigated kinematics flies steadily. Possibly, wing-wing interactions, three-dimensional effects and spanwise flows could significantly help. The present preliminary investigation would hopefully pave the road for further detailed and thorough studies.

\subsection{Introduction}

Flapping motion is advantageous to several low Reynolds number (LRN) applications such as Micro Aerial Vehicles. The combination of the flapping kinematics and the respective viscous fluid dynamics generates complex vortical patterns that help in obtaining the desired force and moment signatures of MAVs and other applications such as swimming robots. 
Several researchers have examined the LRN flapping airfoils and wings fluid phenomena in detail, as mentioned in chapter 6 . Some other studies are devoted to the fluid dynamics of these flows and their dependency on the governing flow and system parameters.

Usually there is a pair of multi-flapping wings, if not more, in MAVs and swimming robots, demanding multi-body investigations. The moving mesh method to resolve the 3-D vortical interactions in these flows is a concern. The respective two-dimensional simulations are also very informative in terms of the 2-D respective phenomena, and a knowledge about their effective governing flow and system parameters help in understanding the effectiveness of these parameters in their respective 3-D flows. However, the multi-body motion in general and multi flapping motions specifically are not trivial to solve, and several recent investigations are devoted to develop mesh motion methods required for these problems. Immersed boundary method [113] is amongst the commonly utilized techniques for multi-body motion studies. However, the implementation of these methods is not a trivial task and difficulty arises in satisfying the boundary layers and continuity and momentum conservations [114].

In this chapter, a multi-body mesh motion method is developed based on the Generalized Grid Interface [132] and layer addition/removal [141]. The motion library can handle multi body figure-of-eight motion (motion in $x$ and $y$ directions) in addition to the rotation of a single and multi moving system of airfoils. The method is validated for some test cases and a few preliminary numerical investigations are performed to show the capability of the developed motion library. In addition, a brief parametric study about the effect of the pitching amplitude of oscillations is conducted to observe the main effects on just lift and drag coefficients. The simulations are carried out in OpenFOAM ${ }^{\circledR}$. 


\subsection{Numerical simulation method}

The purpose of the present chapter is to simulate the figure-of-eight-like flapping motion of two ellipsoidal airfoils ( $2 \%$ of thickness) according to the following equations of motion:

$$
\begin{aligned}
& x_{\text {upper airfoil }}(t)=-R \sin (2 \pi f t), \\
& x_{\text {lower airfoil }}(t)=-R \sin (2 \pi f t), \\
& y_{\text {upper airfoil }}(t)=d \cos (2 \pi f t)-\frac{R}{2} \sin (4 \pi f t), \\
& y_{\text {lower airfoil }}(t)=d \cos (2 \pi f t)+\frac{R}{2} \sin (4 \pi f t), \\
& \alpha_{\text {upper airfoil }}(t)=\alpha_{0}-\bar{\alpha} \sin (2 \pi f t+\phi), \\
& \alpha_{\text {lower airfoil }}(t)=\alpha_{0}+\bar{\alpha} \sin (2 \pi f t+\phi),
\end{aligned}
$$

where all the parameters, e.g. $R$, are equivalent to the ones first introduced in Equations 6.2. The schematic view of the airfoils and their kinematics is shown in Fig. 9.1.

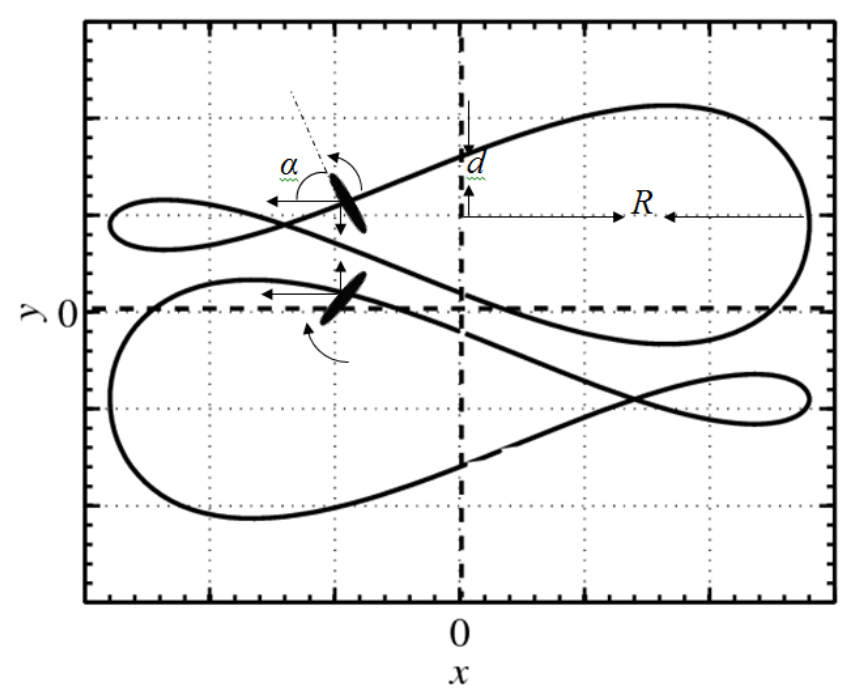

Figure 9.1: Airfoils in the computational domain and their kinematics.

Several steps had to be taken to model and analyze the desired multi-flapping motion in this chapter. First, the GGI algorithm [132] had to be extended to deal with multi-body rotations. This was done in Chapter 7. The accuracy of the GGI method was also inves- 
tigated by simulating a pitching NACA0012 airfoil, studied by Akbari and Price [68] and the respective results were presented in Fig. 7.3. The only concern about this method, is the evaluation of the weighting factors for face-to-face interpolations at the rotating interface (Equations 3.32). If the mesh is not refined enough at the interface, and/or the time resolution is not adequately small, the difference between the interface patches tends to be high. Therefore, it is tried to perform the present simulations with a very refined mesh at the interface and small time steps.

The next step to develop the present motion technique was to extend the layer addition/removal motion algorithm in OpenFOAM ${ }^{\circledR}$, original work by Jasak [141], which was developed to handle multi-body one-dimensional (1-D) motions (either in $x$ or $y$ direction). The developed motion library was then used to simulate the multi-plunging motion of two ellipsoidal airfoils in Chapter 8, and the validation study was presented in Fig. 8.6.

However, the 1-D layer addition/removal method (either in $x$ or $y$ direction) had to be developed to handle 2-D moving cases (in $x$ and $y$ directions), required for figure-of-eight flapping kinematics (Equations 9.1). After extending the original 1-D layer addition/removal algorithm to a 2-D library, the GGI method had to be added into the library, which is successfully done and the next section contains the validation studies performed using it. Appendix A presents the developed code and details about its entries and specifications.

In the following sections, the fluid dynamics of a pair of flapping airfoils is simulated using N-S equations. The computations are based on a second order central differencing scheme for convective and diffusive terms and a second order Euler implicit scheme for temporal discretization, similar to the previous simulations. The resulting linear system of equations is treated with Preconditioned Conjugate Gradient (PCG) solvers, and the Pressure Implicit and Splitting of Operators (PISO) algorithm is used for the pressure-velocity coupling. 


\subsection{Evaluation of the results}

In addition to the above-mentioned validation studies, the developed motion library is evaluated for two flapping validation cases: a harmonic flapping airfoil governed by Equations 6.6 and a figure-of-eight-like flapping airfoil governed by Equations 6.2. An equivalent mesh size $\left(50 \times 10^{3}\right)$ is utilized as the one used in Chapter 6. Time resolution, however, is set to 4000 time steps per cycle, unlike the previous resolution of 2000 time steps in a period. After adding the second dimension in the layer addition/removal method, 2000 times steps were not enough to achieve convergence, meaning that 2000 time steps either ended up with very high Courant numbers (Equation 3.19), hence instability in the force coefficients and/or divergence, and/or simply led to failure of the motion library. The other difference between the validation test cases in Chapter 6 and the present cases is the far-field boundary which resembles a square instead of the circular far-field boundary in Fig. 6.1.

Figure 9.2 shows the obtained $C_{l}$ versus $\tau$ using the developed motion library compared with the results of Amiralaei et al. [6], Bos et al. [88] and Wang et al. [58], for a harmonically flapping airfoil (2\% of thickness) following Equations 6.6.

As can be seen in Fig. 9.2, the peaks of $C_{l}$ are either higher or lower than those in the other studies; however, similar differences are also observed between the references used in Fig. 9.2. Moreover, the mean lift coefficients are within an acceptable difference margin (less than $5 \%$ ) comapred to the other studies.

Next, the figure-of-eight motion of the ellipsoidal airfoil governed by Equations 6.2 is simulated. Figure 9.3 shows the comparison between the present simulation and that in Chapter 6 in Fig. 6.11.

Similar to the observations in Fig. 9.2, the results achieved from the developed algorithm are found to be in good agreement with those obtained utilizing Laplace mesh motion algorithm [6]. Therefore, the present developed motion library is considered to be accurate

enough for the following multi-flapping studies. It is worth noting that no, multi-flapping validation case is studied in this chapter due to the lack of the relevant data in the literature. 


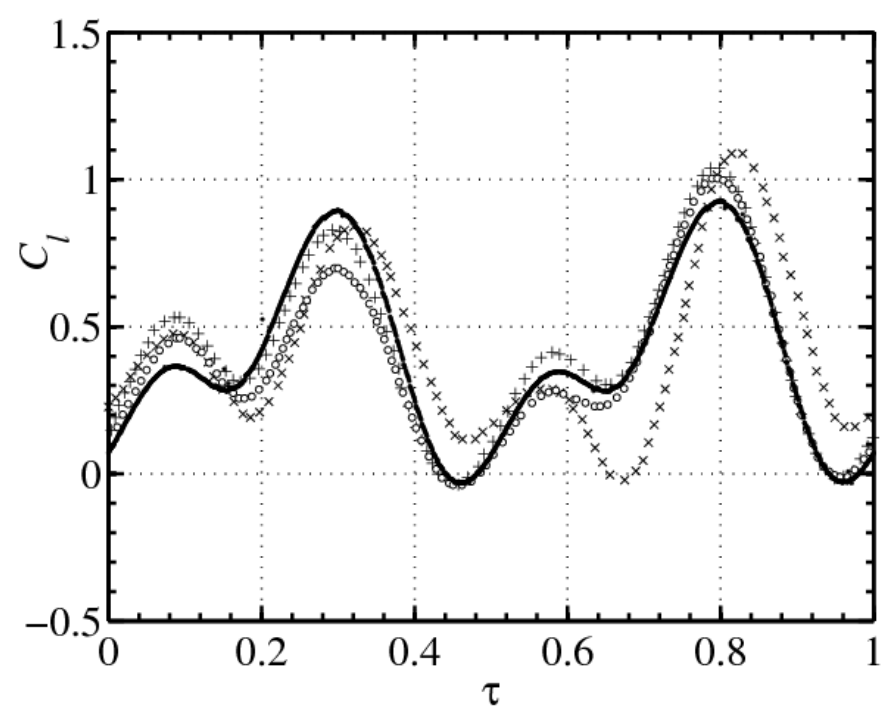

Figure 9.2: Instantaneous lift coefficient $\left(C_{l}\right)$ versus nondimensional time $(\tau)$ of for the flapping motion following Equations 6.6: (-), present simulation with the developed library; (+), Bos et al. [88]; (×), Wang et al. [58]; (o), Amiralaei et al. [6] using Laplace mesh motion algorithm.

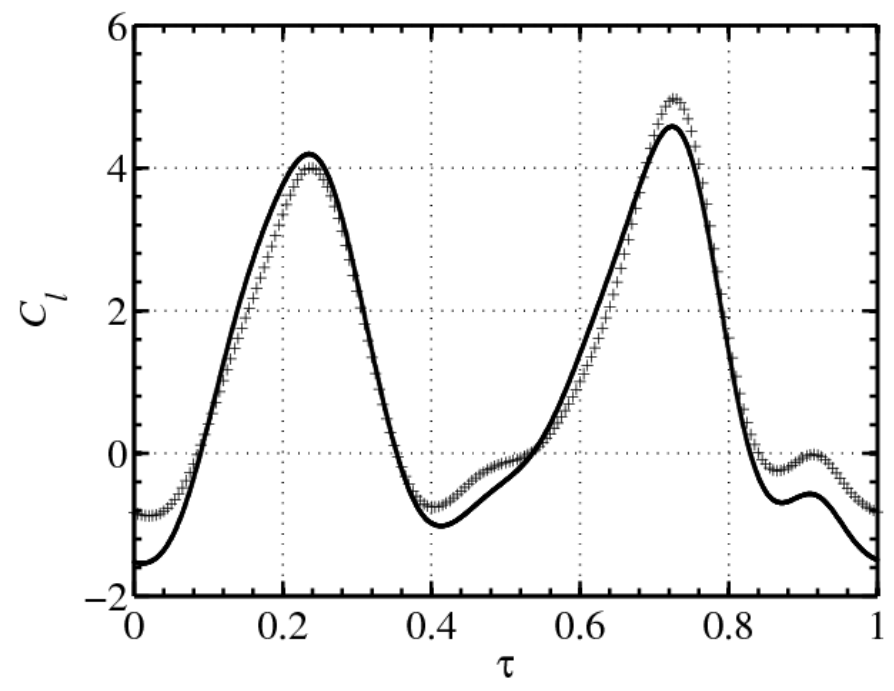

Figure 9.3: Instantaneous lift coefficient $\left(C_{l}\right)$ versus nondimensional time $(\tau)$ for $R=1.4 c$, $d=0.7 c, f=0.25, \alpha_{0}=90^{\circ}, \bar{\alpha}=45^{\circ}, \phi=0$ and $\operatorname{Re}=75$ following Equations 6.2: $(-)$, present simulations with the developed motion library; $(+)$, Amiralaei et al. [6].

\subsection{Results and discussion}

Two thin ellipsoidal airfoils, each one with $t / c=2 \%$, are simulated in this chapter. The airfoils motion are governed by Equations 9.1, and the schematic of the computationl domain 
is shown in Fig. 9.4.

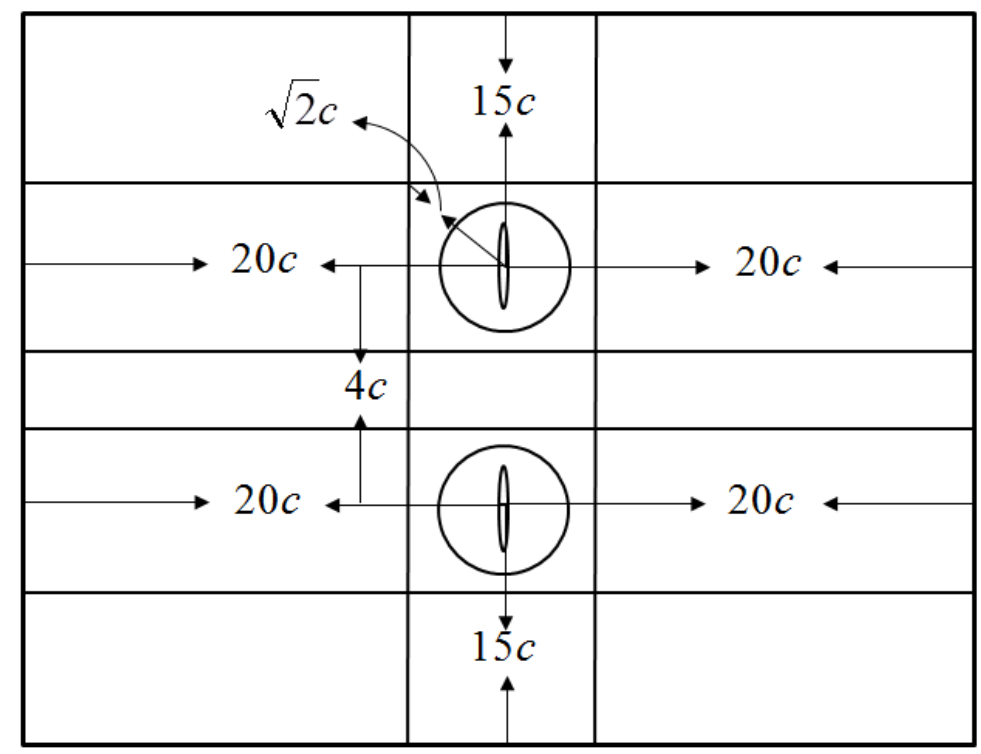

Figure 9.4: Schematic of the airfoils and the computational domain.

The computational domain is extended to $20 c$ and $15 c$ from the airfoils' centers in the $x$ and $y$ directions, respectively. Each airfoil's square-like bounding box (Fig. 9.4) is at a distance of one chord-length from its airfoil's center. This bounding box is required for performing the layer addition/removal portion of the flapping motion. The airfoils' centerto-center spacing is $4 c$. The square regions surrounding the airfoils are discretized using an O-type mesh, while regular quadratic mesh is used for the remaining of the computational domain. The far-field boundary is set to a symmetry condition and the airfoils' surfaces are set to a no-slip boundary condition. Similar to the single figure-of-eight flapping airfoil studied in Chapter 6, the domain within one chord-length from the airfoil surface is discretized using 8800 (176 tangentially $\times 50$ radially) cells and is graded both radially and tangentially (from LE/TE to the mid-chord). The radial grading is such that the distance between the center of the first layer and the airfoil surface is $0.002 c$. The evaluation of the lift and drag coefficient follows the same method presented in Section 6.4.

Three cases are simulated where $\bar{\alpha}=15^{\circ}, 30^{\circ}$ and $45^{\circ}$. Other governing flow and system parameters in Equations 9.1 are fixed to $R=1.4 c, d=0.7 c, f=0.25[\mathrm{~Hz}], \alpha_{0}=90^{\circ}, \phi=0$ 
and $\operatorname{Re}=37.5$. Figures 9.5 and 9.6 show, respectively, the obtained $C_{l}$ and $C_{d}$ versus $\tau$ for the upper airfoil in the computational domain (Fig. 9.4) at $\bar{\alpha}=15^{\circ}$.

Figure 9.5 shows $C_{l}$ versus $\tau$ for the upper airfoil at $\bar{\alpha}=15^{\circ}$. As can be seen, the instantaneous lift coefficients are repeated with constant amplitude after $\tau=6$, showing that the transient variations in the solution have faded and the solution reaches the desired steady state. The airfoil generates downward lift for the first half-stroke $(\tau<0.5)$ with $C_{l_{\max }} \approx$ -1.7. Upward lift is generated for the second half-stroke $(0.5<\tau)$ with two major peaks of $C_{l_{\max }}=0.6$ and 1.5 at $\tau=0.7$ and 0.9 , respectively. The upper airfoil flapping equation (Equations 9.1) resembles that of the figure-of-eight airfoil studied in Chapter 6. However, all the $C_{l}$ curves in Chapter 6 have positive first and second $C_{l_{\max }}$, while in Fig. 9.5 the first half-stroke has a negative $C_{l_{\max }}$, which is even higher than that of the second half-stroke. The study over how the vortical interaction affect the forces is a potential future work. Figure 9.6 also shows $C_{d}$ versus $\tau$ for the upper airfoil at $\bar{\alpha}=15^{\circ}$. Comparison of the peaks of the drag coefficient with those of $C_{l}$, Fig. 9.5, shows that the dominant force coefficient on the upper airfoil is drag. Moreover, the airfoil generates a very small thrust (negative drag coefficient) for $0.4<\tau<0.6$.

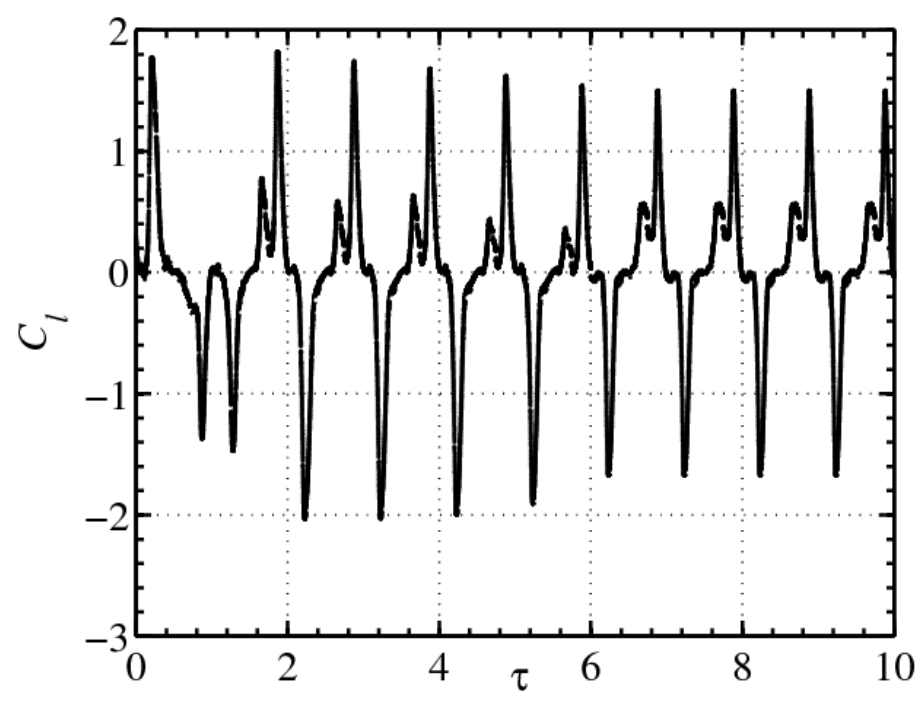

Figure 9.5: Instantaneous lift coefficient $\left(C_{l}\right)$ versus nondimensional time $(\tau)$ for the upper airfoil at $\bar{\alpha}=15^{\circ}$. 


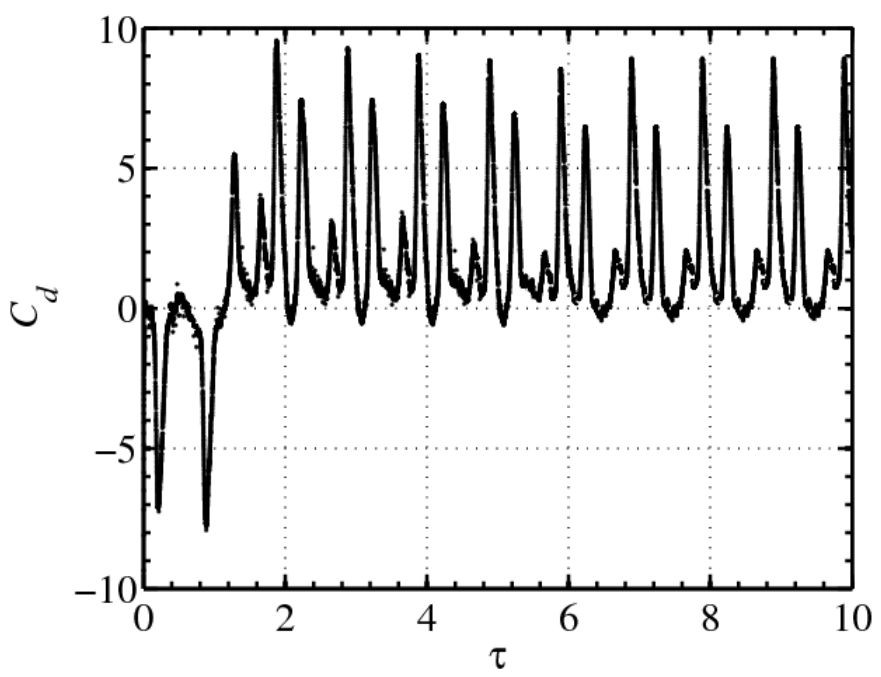

Figure 9.6: Instantaneous drag coefficient $\left(C_{d}\right)$ versus nondimensional time $(\tau)$ for the upper airfoil at $\bar{\alpha}=15^{\circ}$.

Figure 9.7 shows the $C_{l}$ versus $\tau$ for the lower airfoil at $\bar{\alpha}=15^{\circ}$. Unlike the upper airfoil, the lower one has positive lift for the first half-stroke $(\tau<0.5)$ and negative $C_{l}$ for the second half. The magnitudes of peaks of $C_{l}$ are observed to be even higher than those of the upper airfoil. It should be reminded that there are two main differences between the governing equation of motions of the two airfoils. First, the rotations are out of phase, i.e. the upper airfoil rotates counter clock-wise while the lower one rotates clock-wise. Second, as shown in Fig. 9.1 the direction of the $y$-component of the two flapping motions are opposite to each other. In addition to the differences in the equations of motion, the effect of the vortical interactions could be a strong reason for the difference in the $C_{l}$ variations of Figs. 9.5 and 9.7. Figure 9.8 shows $C_{d}$ versus $\tau$ for the lower airfoil at $\bar{\alpha}=15^{\circ}$, where similar to the upper airfoil, higher drag coefficients than lift are observed.

Figures 9.9 and 9.10 are further provided to depict the differences between the $C_{l}$ and $C_{d}$ values of the upper and lower airfoils at $\bar{\alpha}=15^{\circ}$. Only the steady results (after $\left.\tau=6\right)$ are presented. It should be noted that the lift and drag values are shifted downwards for 4 and 10 units, respectively, to ease the comparison.

Two more test cases are investigated to study the effect of the amplitude of the pitching 


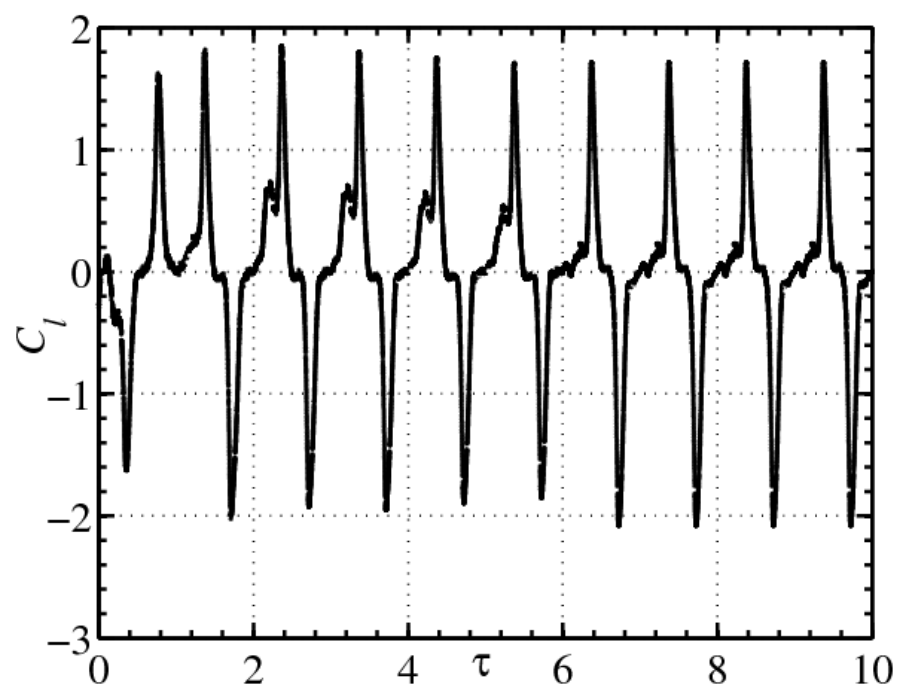

Figure 9.7: Instantaneous lift coefficient $\left(C_{l}\right)$ versus nondimensional time $(\tau)$ for the lower airfoil at $\bar{\alpha}=15^{\circ}$.

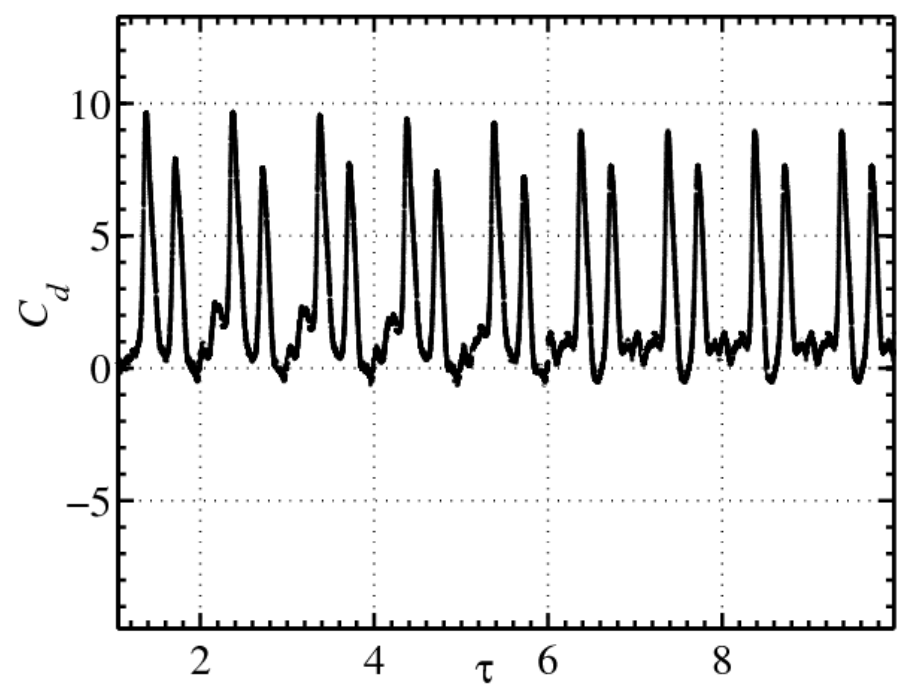

Figure 9.8: Instantaneous drag coefficient $\left(C_{d}\right)$ versus nondimensional time $(\tau)$ for the lower airfoil at $\bar{\alpha}=15^{\circ}$.

oscillations $(\bar{\alpha})$ on the lift and drag of the lower airfoil. The lower airfoil is chosen as according to the solution of single figure-of-eight-like flapping motion, the separated vortices from the airfoil move downward. Fig. 2.1 presents the single figure-of-eight flapping airfoil studied in Chapter 6, showing the direction the separated vortices move. Fig. 9.11 shows $C_{l}$ versus $\tau$ for the lower airfoil at $\bar{\alpha}=30^{\circ}$. Similar to Fig. 9.7, positive lift coefficients are obtained 


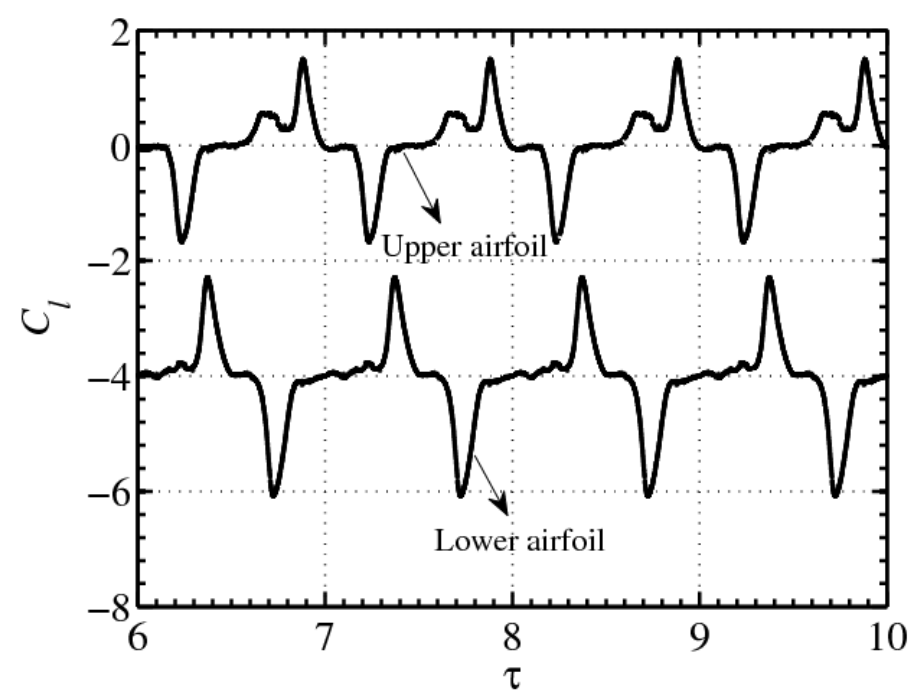

Figure 9.9: Instantaneous lift coefficient $\left(C_{l}\right)$ versus nondimensional time $(\tau)$ for the upper and lower airfoils at $\bar{\alpha}=15^{\circ}$, the lower airfoil $C_{l}$ are shifted 4 units downward.

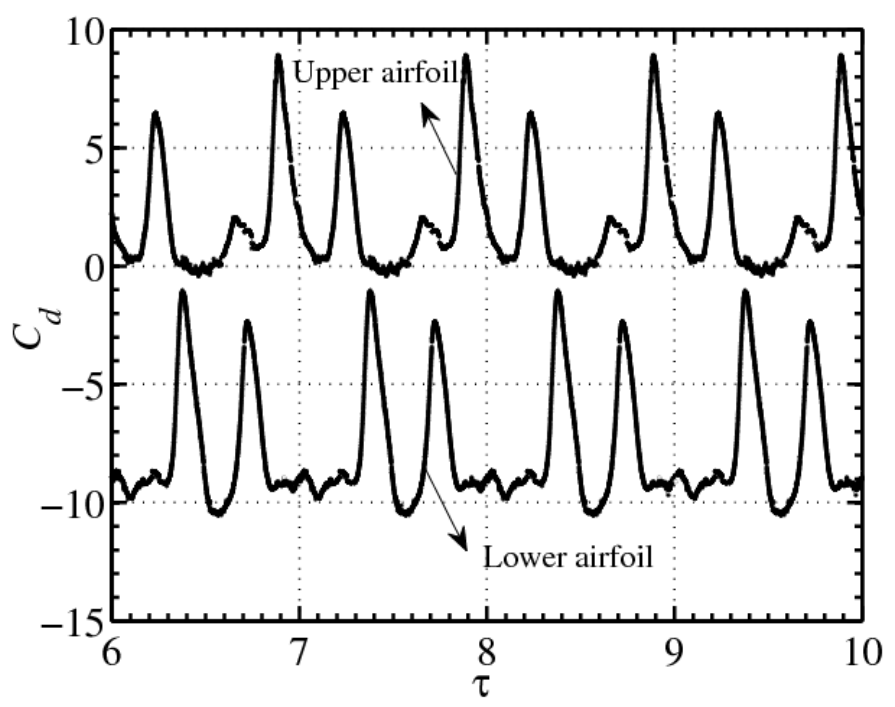

Figure 9.10: Instantaneous drag coefficient $\left(C_{d}\right)$ versus nondimensional time $(\tau)$ for the upper and lower airfoils at $\bar{\alpha}=15^{\circ}$, the lower airfoil $C_{d}$ are shifted 10 units downward.

in the first half-stroke while negative values are obtained in the second half. Comparing the peaks of the lift coefficient shows that the first maximum lift coefficient $\left(C_{l_{\max }}\right)$ is not changed noticeably between $\bar{\alpha}=15^{\circ}$ and $30^{\circ}$. The $C_{l_{\max }}$ is obtained with a lead at $\tau=$ 0.4 and 0.2 for $\bar{\alpha}=15^{\circ}$ and $30^{\circ}$, respectively. The amplitude of the pitching oscillations, however, changes the magnitude of $C_{l_{m i n}}$ in the second half stroke from $\left|C_{l_{m i n}}\right|=2.1$ to 1.6 
at $\bar{\alpha}=15^{\circ}$ and $30^{\circ}$, respectively. This decreasing effect is accompanied with an induced lag in the time at which $C_{l_{\min }}$ is obtained, $C_{l_{\min }}$ is obtained at $\tau=0.7$ and 0.9 at $\bar{\alpha}=15^{\circ}$ and $30^{\circ}$, respectively.

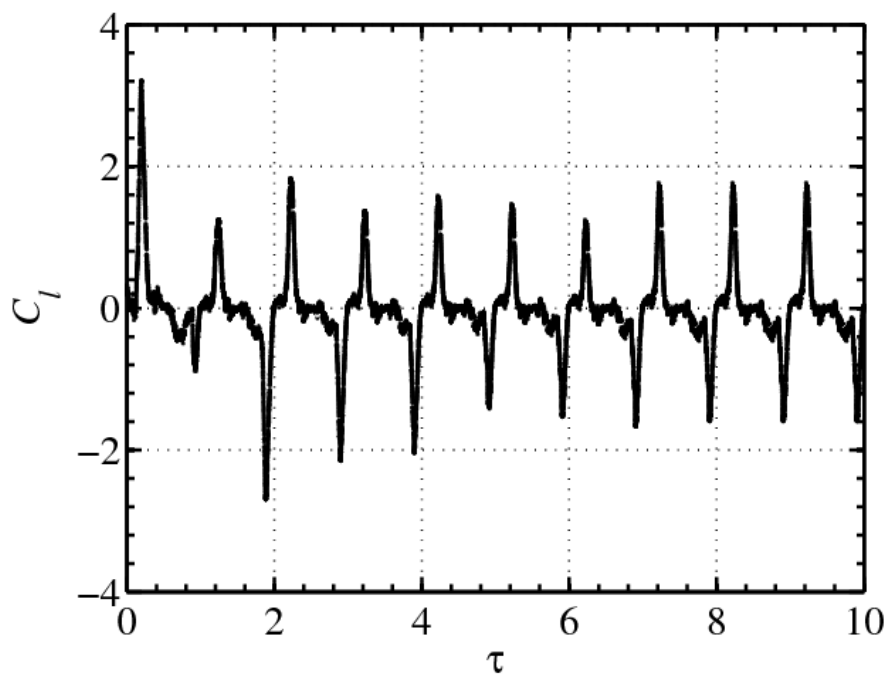

Figure 9.11: Instantaneous lift coefficient $\left(C_{l}\right)$ versus nondimensional time $(\tau)$ for the lower airfoil at $\bar{\alpha}=30^{\circ}$.

Figure 9.12 shows $C_{d}$ versus $\tau$ for the lower airfoil at $\bar{\alpha}=30^{\circ}$. It can be seen that increasing $\bar{\alpha}$ decreases the maximum drag coefficients. The first $C_{d_{\max }}=5.4$ and 3.5 at $\bar{\alpha}$ $=15^{\circ}$ and $30^{\circ}$, respectively. However, $\bar{\alpha}$ does not induce lead or lag into the location of the first $C_{d_{\max }}$, both maximums are obtained at $\tau=0.2$. The second $C_{d_{\max }}$ also decreases from $\bar{\alpha}=15^{\circ}$ and $30^{\circ} . C_{d_{\max }}=9$ and 6 at $\bar{\alpha}=15^{\circ}$ and $30^{\circ}$, where both maximums are obtained at $\tau=0.9$, hence similar to the first $C_{d_{\max }}$ no lead or lag is induced. Moreover, comparing Figs. 9.8 and 9.12 shows that higher thrust $\left(C_{d}<0\right)$ is generated at $\bar{\alpha}=30^{\circ}$ than $\bar{\alpha}=15^{\circ}$.

Finally, $C_{l}$ and $C_{d}$ versus $\tau$ are shown in Figs. 9.13 and 9.14, respectively, for the lower airfoil for $\bar{\alpha}=45^{\circ}$. Unlike, the previous two cases $\left(\bar{\alpha}=15^{\circ}\right.$ and $\left.\bar{\alpha}=30^{\circ}\right)$, where $C_{l}$ values were positive in the first half and negative in the second half for $\bar{\alpha}=15^{\circ}$ and $\bar{\alpha}=30^{\circ}$, positive and negative $C_{l}$ values exist in both first and second half-strokes. This different lift variation is also accompanied with decreased $C_{l_{\max }}$, Fig. 9.13. Figure 9.13 is obtained for the same governing condition as those of $\mathrm{Re}=37.5$ in Fig. 6.24 (Chapter 6). However, it is seen in 


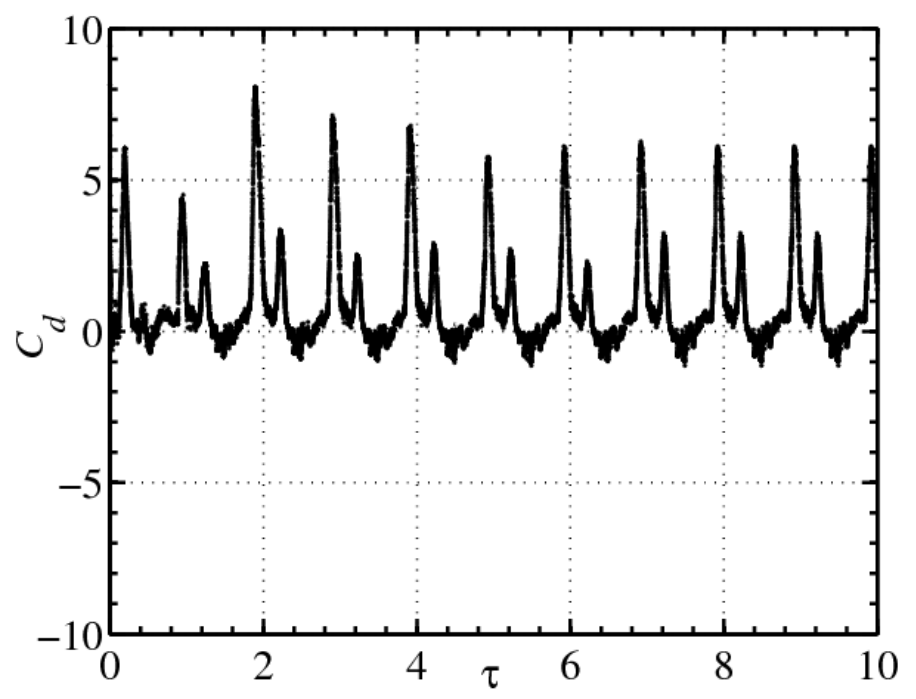

Figure 9.12: Instantaneous drag coefficient $\left(C_{d}\right)$ versus nondimensional time $(\tau)$ for the lower airfoil at $\bar{\alpha}=30^{\circ}$.

Fig. 9.13 that significant downward lift coefficients are obtained. Moreover, noticeable drag force is exerted on the airfoil, Fig . 9.14. This means that the 2-D airfoil-airfoil interactions deteriorate the performance of the airfoils, or at least the lower airfoil.

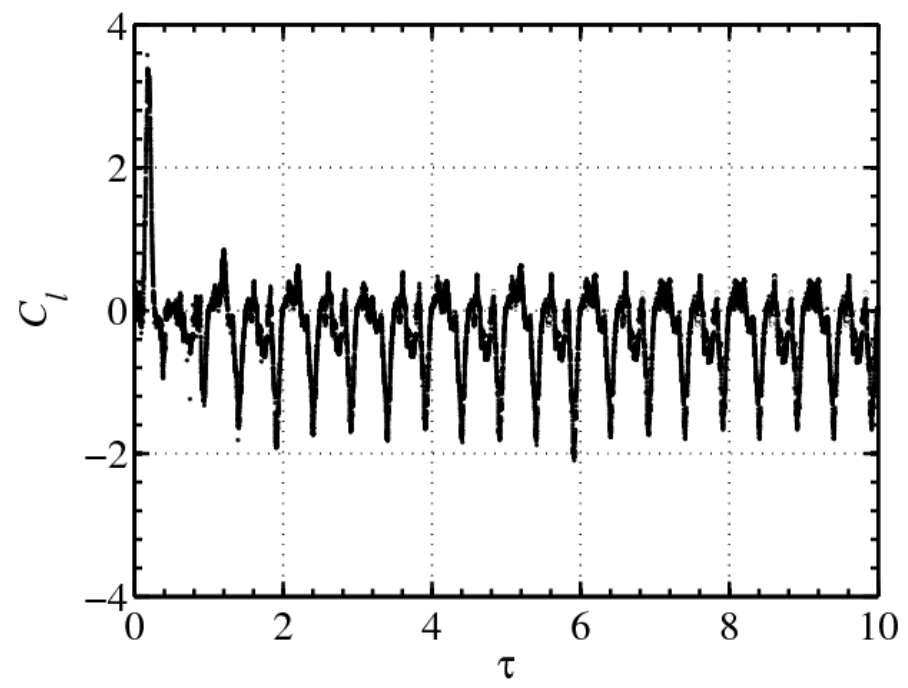

Figure 9.13: Instantaneous lift coefficient $\left(C_{l}\right)$ versus nondimensional time $(\tau)$ for the lower airfoil at $\bar{\alpha}=45^{\circ}$.

Figure 9.14 shows $C_{d}$ versus $\tau$ for the lower airfoil at $\bar{\alpha}=45^{\circ}$. First, higher thrust than the $\bar{\alpha}=15^{\circ}$ and $30^{\circ}$ cases is generated at $\bar{\alpha}=45^{\circ}$. The first $C_{d_{\max }}$ increases from $C_{d_{\max }}=$ 
3.5 to 4 at $\bar{\alpha}=30^{\circ}$ and $45^{\circ}$, respectively, accompanied with an induced lag at $\tau=0.2$ and 0.4 at $\bar{\alpha}=30^{\circ}$ and $45^{\circ}$. The second $C_{d_{\max }}$ at $\bar{\alpha}=15^{\circ}$ and $30^{\circ}$, however, is converted to a maximum thrust coefficient $\left(C_{d_{\max }}<0\right)$, but obtained at the same $\tau(\tau=0.9)$.

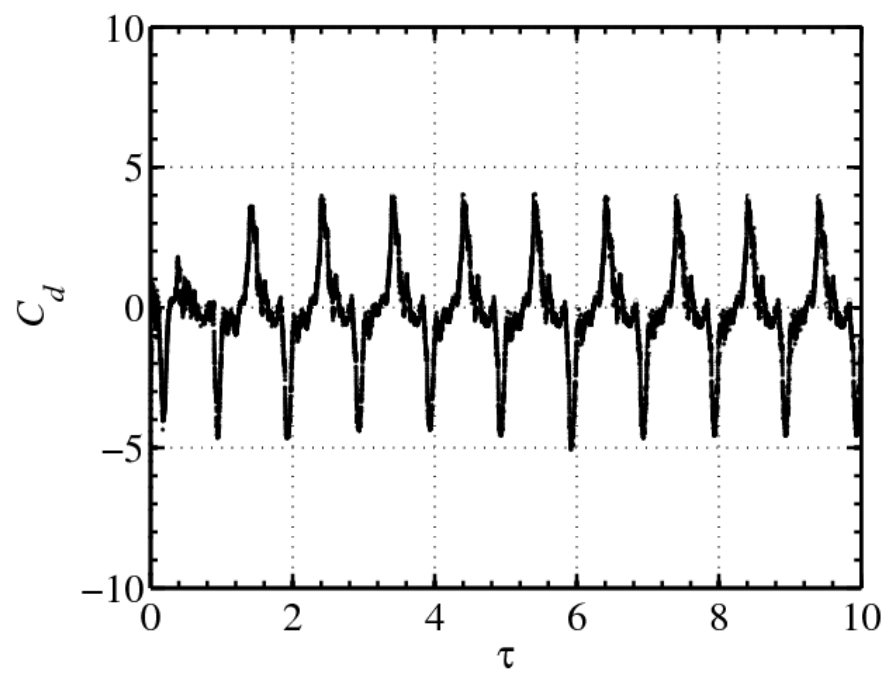

Figure 9.14: Instantaneous drag coefficient $\left(C_{d}\right)$ versus nondimensional $(\tau)$ for lower airfoil at $\bar{\alpha}=45^{\circ}$.

\subsection{Summary and conclusion}

In this chapter, a mesh motion library is developed based on the layer addition/removal [141] and Generalized Grid interface (GGI) [132] algorithms. The developed motion algorithm is used for the multi figure-of-eight flapping airfoils simulations. The algorithm is first used to study single harmonic and figure-of-eight flapping airfoils, for which previous studies were performed using Laplace mesh motion technique. The results of the developed motion library are found to be in good agreement with those of the Laplace algorithm when the mean values of the force coefficients are compared. The main differences between the results are observed around the peaks of the force signatures, where the discrepancy is less than $5 \%$. Furthermore, the method is used to simulate the figure-of-eight flapping motion of two airfoils. A few numerical examples are presented for different amplitudes of pitching 
oscillations to show the capability of the developed method in handling the multi flapping motions, to investigate the lift and drag coefficients of both airfoil in one case and to study the effect of the pitching amplitude of oscillations on the lift and drag coefficients of one of the airfoils. In all the studied cases, the drag is significantly higher than the lift, showing how the airfoil-airfoil interaction changes the instantaneous lift and drag coefficients when compared to those of a single flapping airfoil. 


\section{Chapter 10}

\section{Concluding Remarks}

The growing interest in low Reynolds number (LRN) flow discipline emerges from their corresponding state-of-the-art applications such as Micro Aerial Vehicles (MAVs), swimming robots and off-shore structures. The respective fluid dynamics of these applications is highly viscous, accompanied with complex vortical patterns affecting the performance and flow characteristics. The inherent complexity of these flows rises at unsteady conditions, demanding successful modeling to meet robust design targets. Fortunately, the main governing fluid phenomena are known in terms of their mechanisms. However, in spite of the recent research endeavors, the tangible gap between the existing knowledge and the respective design constraints needs more thorough studies. For example, the behavior of the fluid phenomena under different governing conditions is not yet well understood.

Forced and flow-induced oscillations of LRN airfoils, wings and geometries (in general) in single and multi-body configurations are amongst highly challenging problems to solve in terms of the computational time, accuracy and appropriate methods to perform the desired kinematics. The existing analytical tools are not sufficiently accurate to capture the required details of these flows. On the other hand, although experimental means stand out as very accurate techniques, they are very time consuming and expensive to conduct a thorough study. Hence, Computational Fluid Dynamics (CFD), also accurate, is an excellent candi- 
date for LRN flows.

The focus of this thesis is on the CFD study of force-induced oscillations of two-dimensional (2-D) airfoils in both single and multi-moving arrangements, where pitching, plunging and novel figure-of-eight-like flapping airfoils are investigated. The motivation of the performed studies is a rotating-flapping mechanism which uses two blades for generating its required unsteady forces in a stable hovering flight. Thorough parametric studies corresponding to this application are conducted to contribute to the current LRN flow knowledge, and multibody motion algorithms are developed for handling multi-pitching, plunging and flapping airfoils.

In the single airfoil studies in Chapters 4, 5 and 6, pitching, plunging and a novel figureof-eight-like flapping kinematics, respectively, are investigated. The conducted parametric studies are mainly focused on the instantaneous force signatures, where in some cases of interest pitching moment and flow structures surrounding the airfoil are also investigated. The high lift generation of the studied figure-of-eight flapping pattern is favorable in LRN flight, where this kinematic may be used instead of the common flapping patterns, e.g. in MAVs.

In the multi airfoils studies in Chapters 7, 8 and 9, appropriate mesh motion methodologies are developed based on the so-called dynamic layering and Generalized Grid Interface (GGI) algorithms to perform multi moving-airfoil unsteady problems. Although the purpose of developing these methodologies is to study paired-airfoil problems, they can also be applied to more than two moving objects with any kinematics different than those studied in this research. Moreover, the developed methodologies may be used for single moving objects with a significant gain in the computational time.

\subsection{Future Work}

There are a number of possible future work which are categorized as the following: 
- The developed motion library is used for the fluid dynamic simulations of airfoils. Fluid-structure interaction is the most important extension to this work. As the final target would be a multi-disciplinary design of an application, dynamics or flight dynamics of the system could also be modeled, where all these disciplines require an appropriate interface for the aerodynamic, structure and flight dynamic models to be coupled. This eventually could result in a powerful optimization workflow for design improvement or to start a design from scratch, where one could introduce some given parameters in the optimization algorithm and extract accurate results as the outputs.

- Although experimental studies are extremely expensive, the multi-moving tests are highly recommended. The present literature lacks a real-world insight about multibody systems.

- The developed motion library needs improvement to achieve more robustness, where robustness is defined as to minimize the user interaction and expertise needed to set up a test case. Further research is needed in that direction.

- Three-dimensional numerical and experimental studies of pitching, plunging and flapping wings in both single and multi configurations are scarce in the literature.

- The application of Artificial Neural Networks (ANNs) and Adaptive Neuro-fuzzy Inference System (ANFIS) is recommended for both 2-D and 3-D unsteady LRN studies to develop tools for rapid prediction of fluid forces under different conditions.

- More extensive research about multi-body airfoils can be conducted, as there is limited data in the literature.

\subsection{Thesis Publications}

The publications resulted in this work are listed as the following: 


\subsubsection{Published Chapters in Books}

- Amiralaei. M. R., Alighanbari, H., and Hashemi, S.M. (2011) Investigation of low Reynolds number unsteady flow around airfoils in pitching, plunging and flapping motions. Chapter in book in CFD Applications in Energy and Environment Sectors.

- Alighanbari, H., Amiralaei. M. R., and Savtchenko, S. (2011) Experimental, analytical and numerical investigation of a swirling submerged jet flow. Chapter in book in CFD Applications in Energy and Environment Sectors.

\subsubsection{Refereed Journal Publications}

- Amiralaei. M. R., Alighanbari, H., and Hashemi, S. M. (2011) On the force signatures of a pair of multi-plunging airfoils. Proceedings of the Institution of Mechanical Engineers, Part G: Journal of Aerospace Engineering. Accepted, JERO1287.

- Amiralaei. M. R., Alighanbari, H., and Hashemi, S. M. (2011) Flow field characteristics of a flapping airfoil using computational fluid dynamics. Journal of Fluids and Structures. 27: 1068-1085.

- Amiralaei. M. R., Alighanbari, H., and Hashemi, S. M. (2010) An investigation into the effects of unsteady parameters on the aerodynamics of a low Reynolds number pitching airfoil. Journal of Fluids and Structures. 26: 979-993.

- Amiralaei. M. R., Alighanbari, H., and Hashemi, S. M. (2010) Effects of Reynolds number and pitching amplitude of oscillation on the aerodynamics of a flapping airfoil. Journal of Mathematics in Engineering, Science and Aerospace. 1: 1-13.

\subsubsection{Submitted Journal Publications}

- Amiralaei. M. R., Alighanbari, H., and Hashemi, S. M. (2011) Numerical modelling of a low Reynolds number plunging airfoil flow field characteristics. Proceedings of the 
Institution of Mechanical Engineers, Part G: Journal of Aerospace Engineering. Under Review, JAERO1305.

- Adique, M., Amiralaei. M. R., and Alighanbari, H. (2011) Studies in neural network application for low Reynolds number unsteady flow around an airfoil in figure-eight motion. Proceedings of the Institution of Mechanical Engineers, Part G: Journal of Aerospace Engineering. Under Review, JAERO1288.

\subsubsection{Refereed Conference Publications}

- Amiralaei. M. R., Alighanbari, H., and Hashemi, S. M. (2010) Unsteady low Reynolds number computational study of an airfoil undergoing a novel figure-eight kinematics. Proceedings of the 40th Fluid Dynamics Conference and Exhibit, AIAA 2010-4457, Chicago, Illinois, USA.

- Amiralaei. M. R., Alighanbari, H., and Hashemi, S. M. (2010) Unsteady CFD of a plunging airfoil in low Reynolds number flow regime. Proceedings of the 40th Fluid Dynamics Conference and Exhibit, AIAA 2010-4460, Chicago, Illinois, USA.

- Amiralaei. M. R., Alighanbari, H., and Hashemi, S. M. (2010) The unsteady aerodynamics of paired plunging airfoils using computational fluid dynamics. Proceedings of the AIAA Guidance, Navigation, and Control Conference, AIAA 2010-8119, Toronto, Ontario, Canada.

- Adique, M., Amiralaei. M. R., and Alighanbari, H. (2010) Application of artificial neural networks in aerodynamics prediction of low-Reynolds-number figure-eight motion of an airfoil. Proceedings of the AIAA Guidance, Navigation, and Control Conference, AIAA 2010-8120, Toronto, Ontario, Canada.

- Amiralaei. M. R., Alighanbari, H., and Hashemi, S. M. (2010) A fluid dynamics study of a modified low-Reynolds number flapping motion, Proceedings of ASME 2010 3rd Joint US-European Fluids Engineering Summer Meeting and 8th Interna- 
tional Conference on Nanochannels, Microchannels, and Minichannels, FEDSM2010ICNMM2010-3037, Montreal, Canada.

- Amiralaei. M. R., and Alighanbari, H. (2010) Experimental and numerical study of a pitching blade. Proceedings of ASME 2010 3rd Joint US-European Fluids Engineering Summer Meeting and 8th International Conference on Nanochannels, Microchannels, and Minichannels, FEDSM-ICNMM2010- 31104, Montreal, Canada.

- Alighanbari, H., Amiralaei. M. R., and Savtchenko, S. (2010) Analytical and numerical study of a swirling submerged jet flow. Proceedings of ASME 2010 3rd Joint USEuropean Fluids Engineering Summer Meeting and 8th International Conference on Nanochannels, Microchannels, and Minichannels, FEDSM-ICNMM2010-30777, Montreal, Canada.

- Amiralaei. M. R., Alighanbari, H., and Hashemi, S. M. (2010) The unsteady Fluid dynamics of a pair of pitching airfoils using computational fluid dynamics. Proceedings of the ASME 2010 International Mechanical Engineering Congress Exposition IMECE2010, IMECE2010-39396, Vancouver, British Columbia, Canada.

- Amiralaei. M. R., and Alighanbari, H. (2010) A parametric study of a three dimensional pitching wing aerodynamics. Proceedings of the ASME 2010 International Mechanical Engineering Congress Exposition IMECE2010, IMECE2010-39400, Vancouver, British Columbia, Canada.

- Amiralaei. M. R., Partovibakhsh, M., and Alighanbari, H. (2010) Application of adaptive networkbased fuzzy inference system (ANFIS) in aerodynamics prediction of low-Reynolds-number flapping motion. Proceedings of the ASME 2010 International Mechanical Engineering Congress Exposition IMECE2010, IMECE2010-40679, Vancouver, British Columbia, Canada.

- Amiralaei. M. R., Alighanbari, H., and Hashemi, S. M. (2009) Computational study of unsteady very low Reynolds number flow around a 2D pitching airfoil. In Proceed- 
ing of Canadian Aeronautics and Space Institute AERO09 Conference Aerodynamics Symposium.

- Amiralaei. M. R., Alighanbari, H., and Hashemi, S. M. (2009) Influence of unsteady parameters on the aerodynamics of a low Reynolds number pitching airfoil. Proceedings of the ASME 2009 International Mechanical Engineering Congress Exposition, IMECE2009-11827, Lake Buena Vista, Florida, USA. 


\section{Appendix A}

\section{Motion Methodology for Multi}

\section{Figure-of-Eight Flapping Motions}

The multi-airfoils simulations in Chapter 7-9 are performed by developing the original mesh motion algorithms in OpenFOAM ${ }^{\circledR}$. The simulations of Chapter 9 correspond to two airfoils under figure-of-eight-like flapping motion, where the developed mesh motion library is a combination of those in Chapters 7 and 8 with further developments. Therefore, only the code for the motion in Chapter 9 is presented here along with some specifications and file settings required to perform the simulations. It should be mentioned that multi pitching or multi plunging of two airfoils can be done using the provided code, if the corresponding settings are changed according to the desired motion.

The following piece of code shows the entries in dynamicMeshDict, the file containing the motion settings for Equations 9.1.

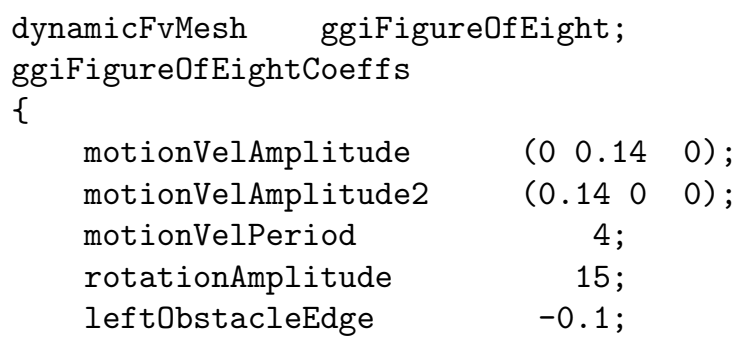




\begin{tabular}{|c|c|c|}
\hline 9 & downObstacleEdge & 0.1 ; \\
\hline 10 & rightObstacleEdge & $0.1 ;$ \\
\hline 11 & upObstacleEdge & 0.3 \\
\hline 12 & lowerUpObstacleEdge & $-0.1 ;$ \\
\hline 13 & lowerDownObstacleEdge & -0.3 \\
\hline 14 & down & \\
\hline 15 & \{ & \\
\hline 16 & minThickness & $0.01 ;$ \\
\hline 17 & maxThickness & 0.05 \\
\hline 18 & \} & \\
\hline 19 & up & \\
\hline 20 & \{ & \\
\hline 21 & minThickness & $0.01 ;$ \\
\hline 22 & maxThickness & 0.05 ; \\
\hline 23 & \} & \\
\hline 24 & left & \\
\hline 25 & \{ & \\
\hline 26 & minThickness & $0.01 ;$ \\
\hline 27 & maxThickness & 0.03 \\
\hline 28 & \} & \\
\hline 29 & right & \\
\hline 30 & \{ & \\
\hline 31 & minThickness & $0.01 ;$ \\
\hline 32 & maxThickness & 0.03 ; \\
\hline 33 & \} & \\
\hline 34 & lowerDown & \\
\hline 35 & \{ & \\
\hline 36 & minThickness & $0.01 ;$ \\
\hline 37 & maxThickness & 0.05 ; \\
\hline 38 & \} & \\
\hline 39 & lowerUp & \\
\hline 40 & \{ & \\
\hline 41 & minThickness & $0.01 ;$ \\
\hline 42 & maxThickness & 0.05 ; \\
\hline 43 & \} & \\
\hline 44 & & \\
\hline
\end{tabular}

The geomterical entries of dynamicMehsDict file are shown in Fig. A.1. The entries showing the left, right, up and down boundaries of each airfoil are necessary for performing the translational motion (in $\mathrm{x}$ and $\mathrm{y}$ directions) of each airfoil. For example, downObstacleEdge in dynamicMeshDict and Fig. A.1, set to 0.1, shows that this edge is as $y=0.1$ in the computational domain.

In the dynamicMeshDict there are other entries, e.g. down, containing maximum and minimum cell layer thicknesses. For example, minThickness of 0.01 means that the cell layer is removed when its thickness reaches 0.01 , or maxThickness of 0.03 means that a new cell 


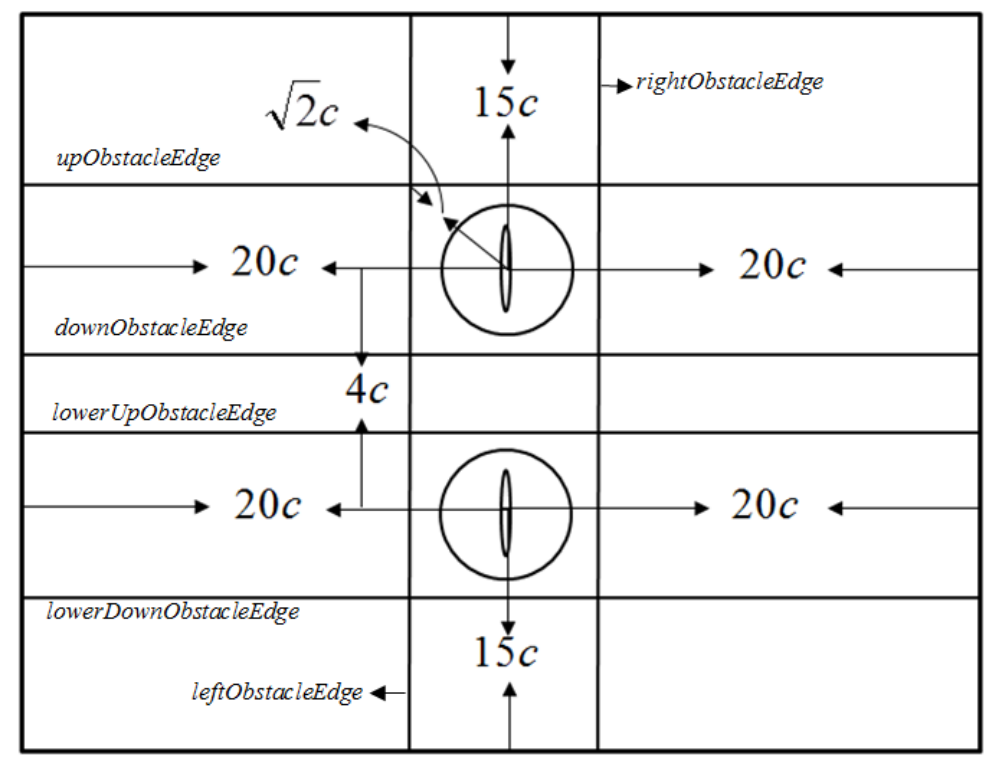

Figure A.1: The schematic of the airfoils and entries in the dynamicMeshDict file.

layer is added when the thickness reaches 0.03. It should be mentioned that according the dynamicMeshDict file the developed motion library is called ggiFigureOfEight. The following code shows ggiFigureOfEight code for performing figure-of-eight-like flapping motion of two airfoils. The following code has been used with the unsteady flow solver in OpenFOAM, icoDyMFoam, and is written in $\mathrm{C}++$. In ggiFigureOfEight. $\mathrm{C}$ code provided in the following, the required header files are called and cells are marked, where this marking is used for the translational motions. For the rotations, two cell regions are also defined. The position of the computational nodes within each circular region are updated considering rotational and translational motions.

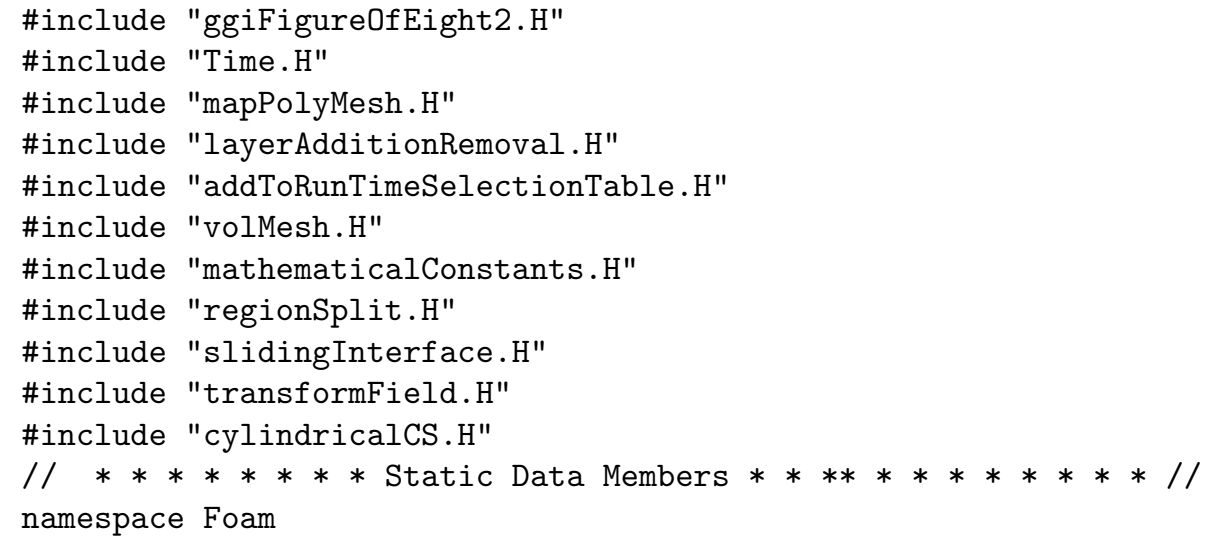




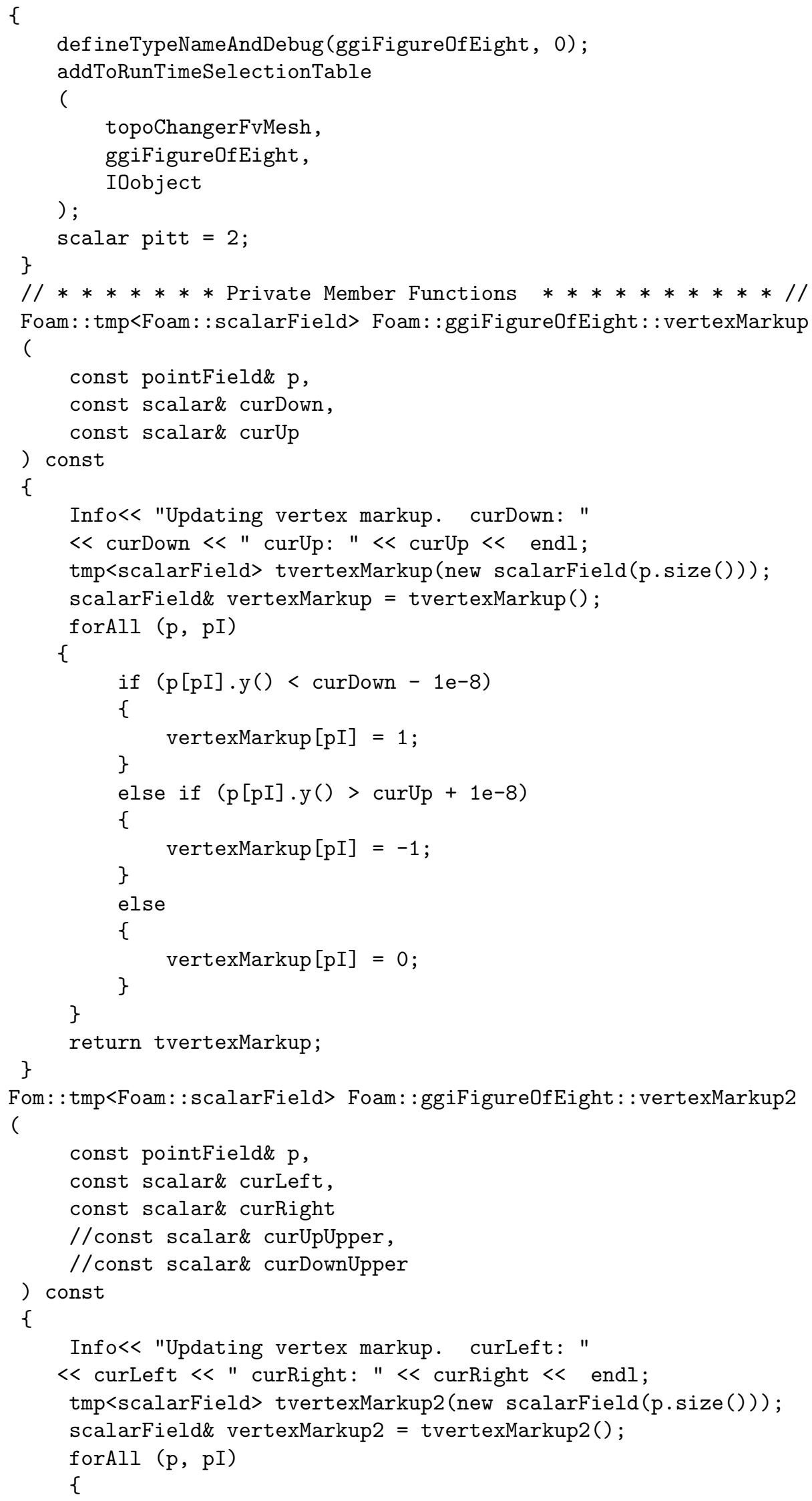




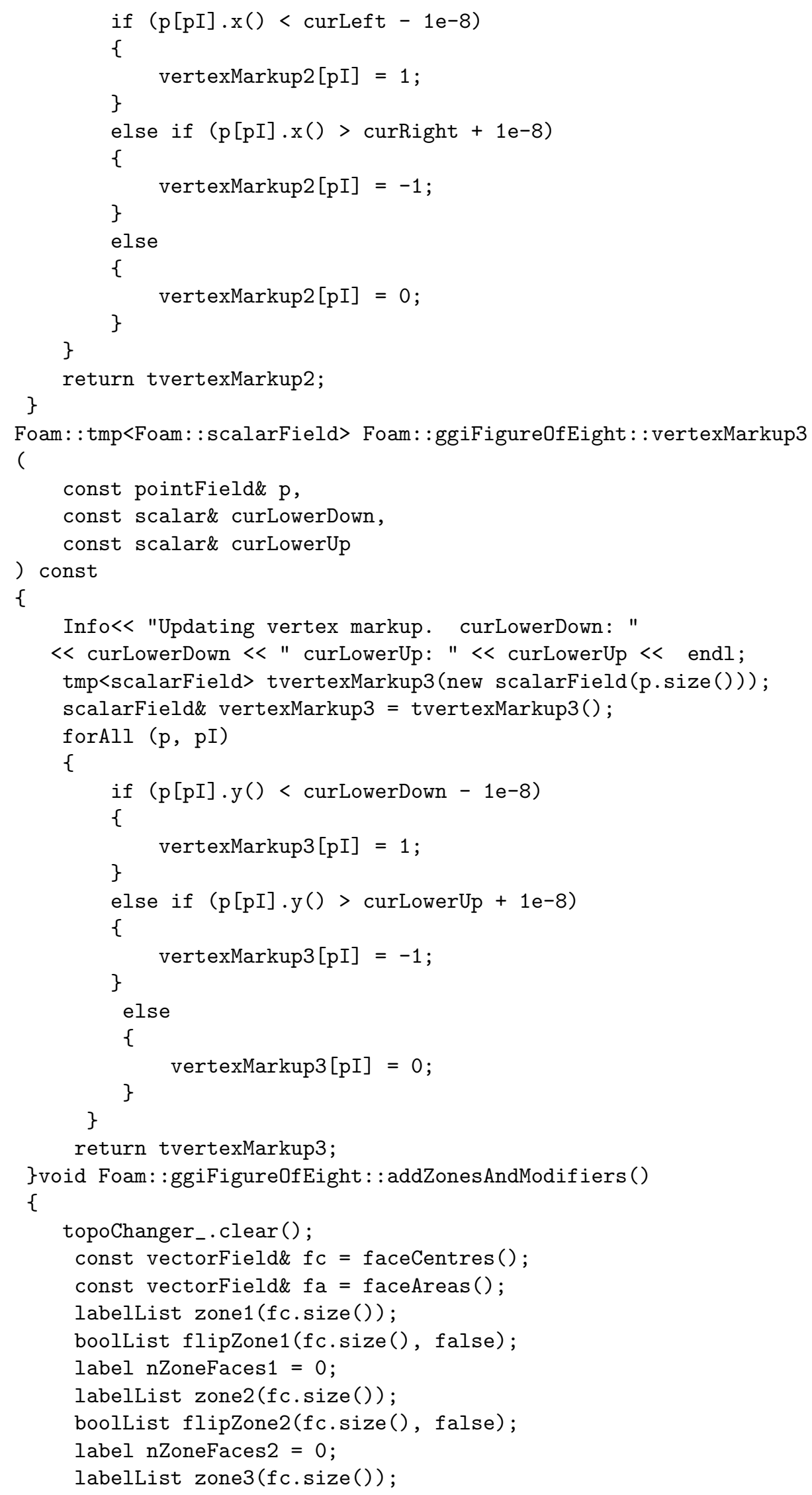




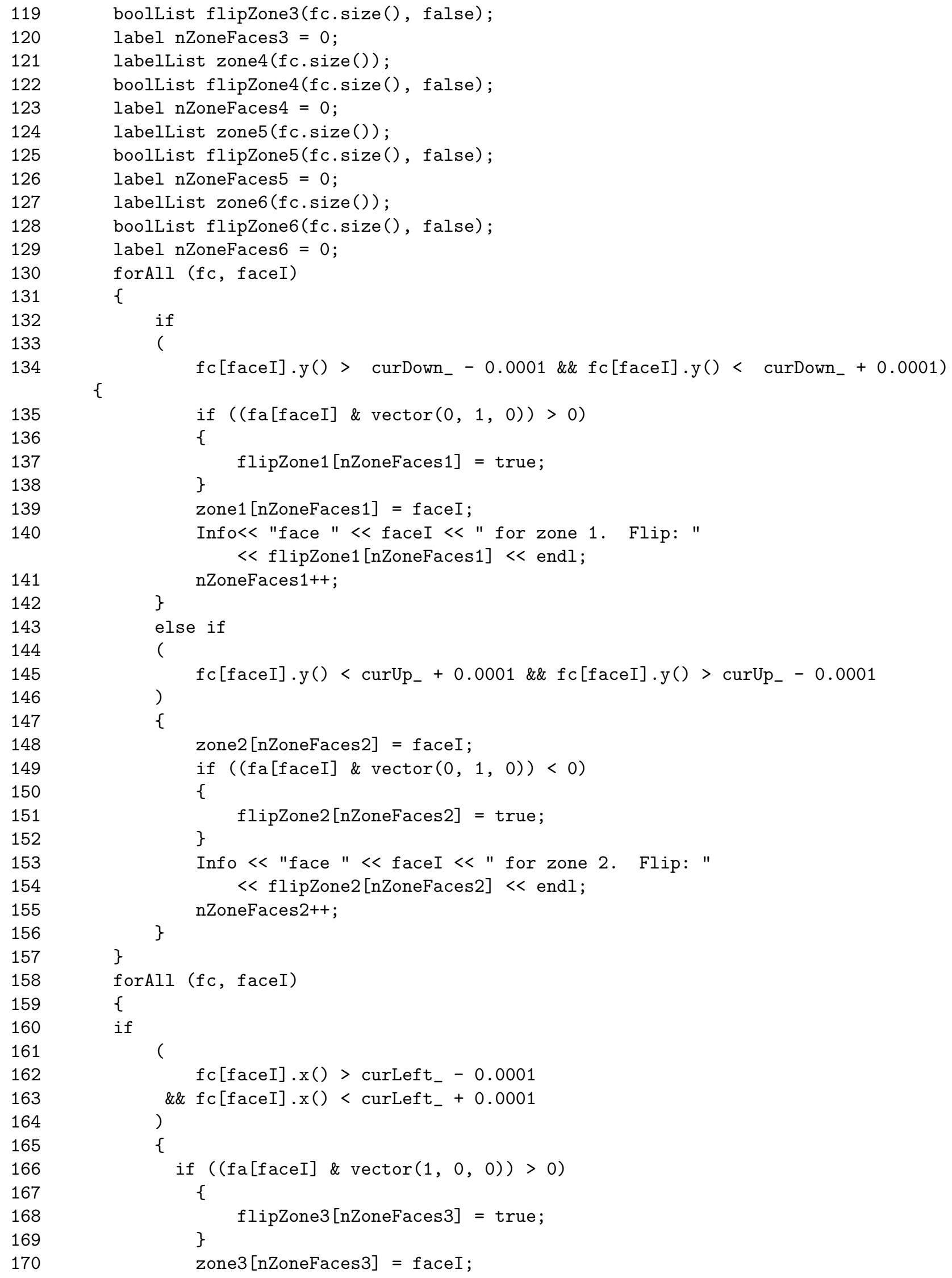




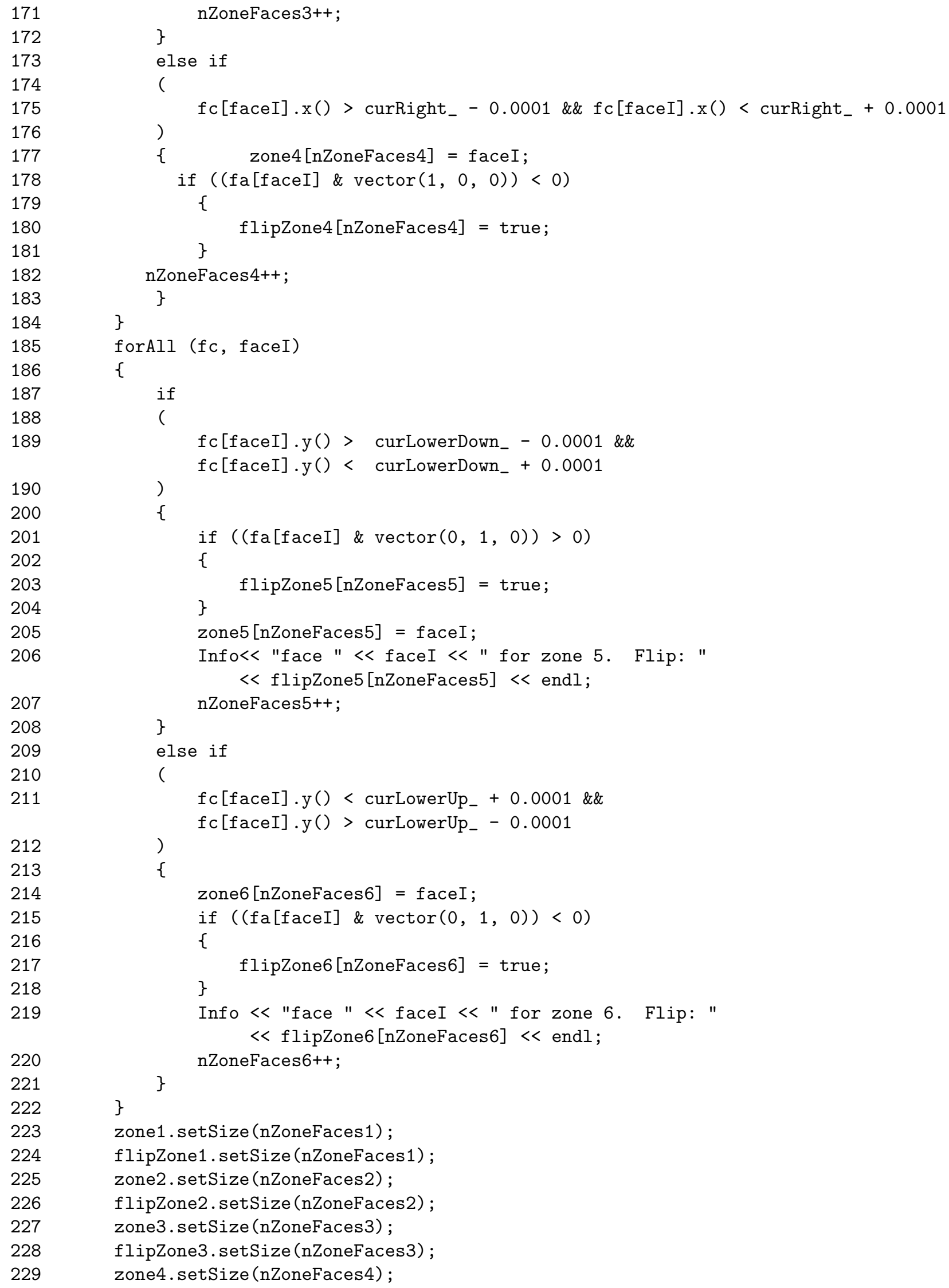




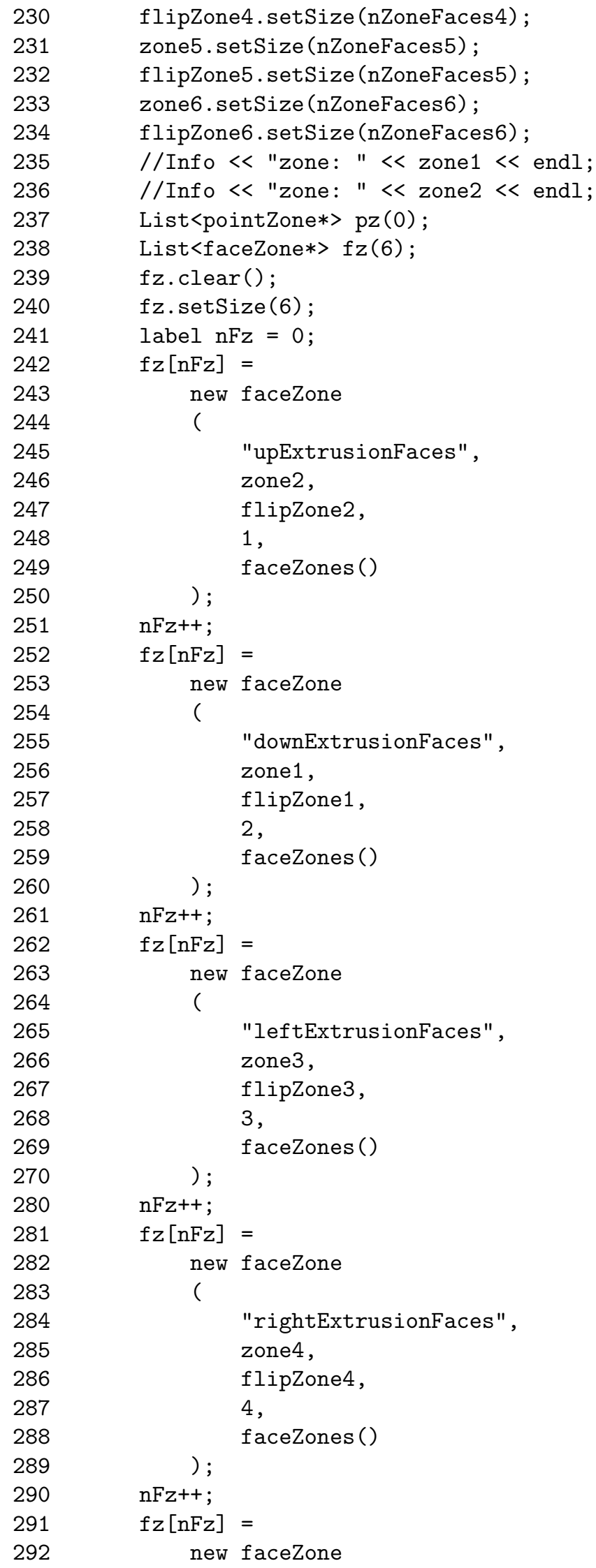

flipZone4.setSize (nZoneFaces4); zone5. setSize (nZoneFaces5); flipZone5. setSize (nZoneFaces5); zone6.setSize (nZoneFaces6); flipZone6.setSize (nZoneFaces6); //Info < "zone: " < zone1 < endl; //Info < "zone: " < zone2 < endl; List<pointZone*> pz $(0)$; List<faceZone $*>\mathrm{fz}(6)$; fz.clear(); fz.setSize(6); label $\mathrm{nFz}=0$; $\mathrm{fz}[\mathrm{nFz}]=$ new faceZone

( "upExtrusionFaces", zone2, flipZone2, 1 , faceZones() ); $\mathrm{nFz++}$; $\mathrm{fz}[\mathrm{nFz}]=$ new faceZone (

"downExtrusionFaces", zone1, flipZone1, 2 , faceZones () ); $\mathrm{nFz++}$; $f z[n F z]=$ new faceZone ( "leftExtrusionFaces", zone3, flipZone3, 3 , faceZones()

); $\mathrm{nFz++}$; $\mathrm{fz}[\mathrm{nFz}]=$ new faceZone ( "rightExtrusionFaces", zone4, flipZone4, 4 , faceZones () ); $\mathrm{nFz++}$; $\mathrm{fz}[\mathrm{nFz}]=$ new faceZone 


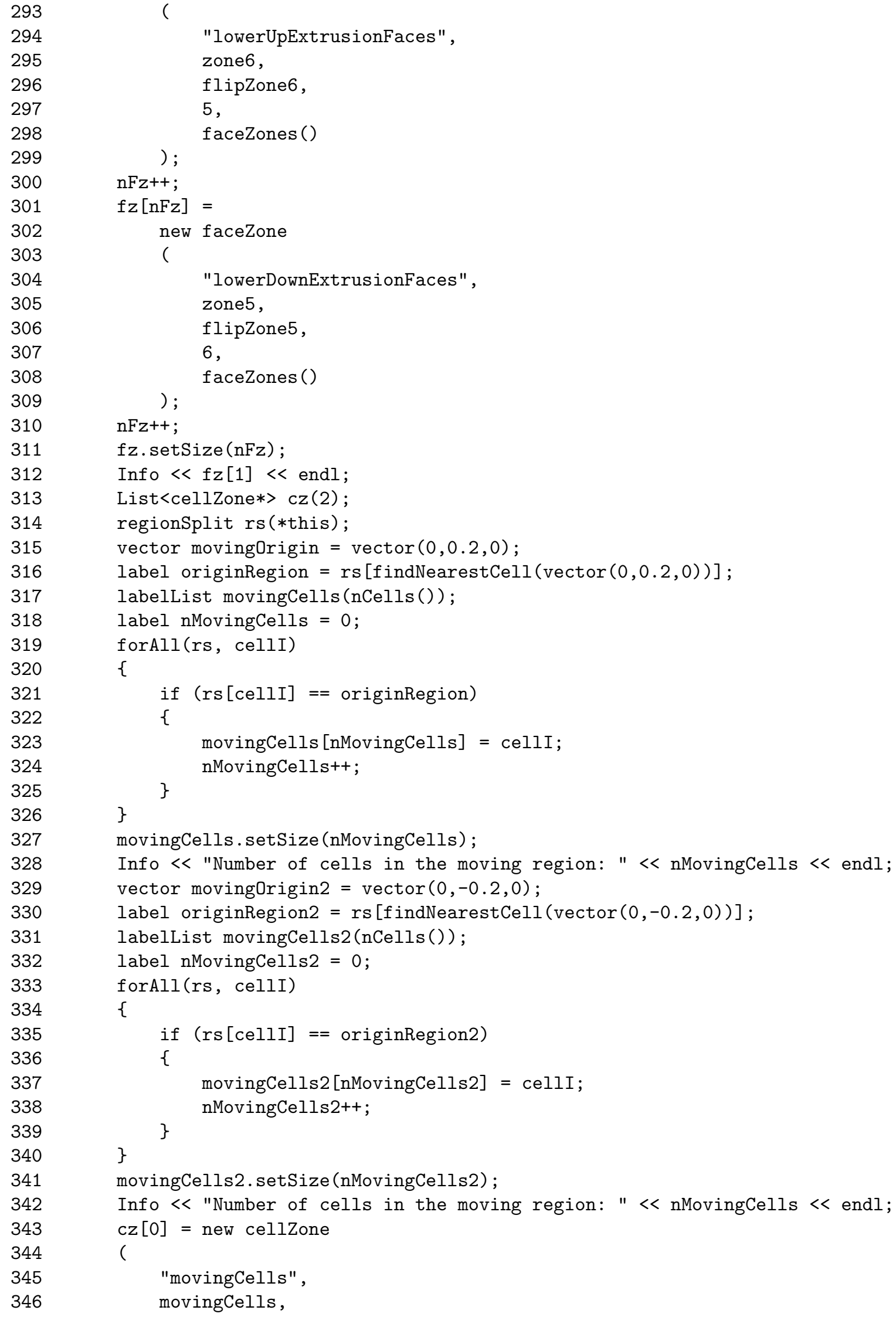




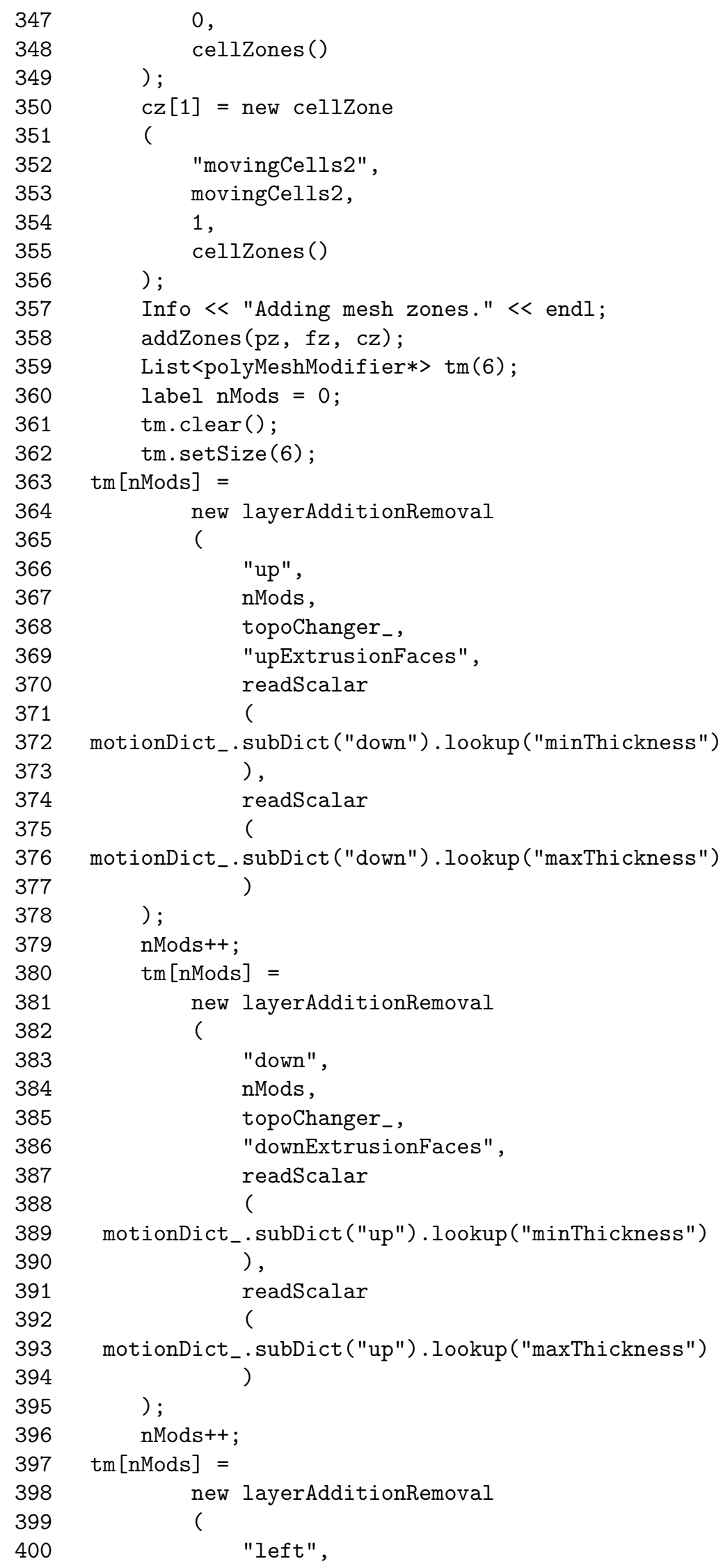




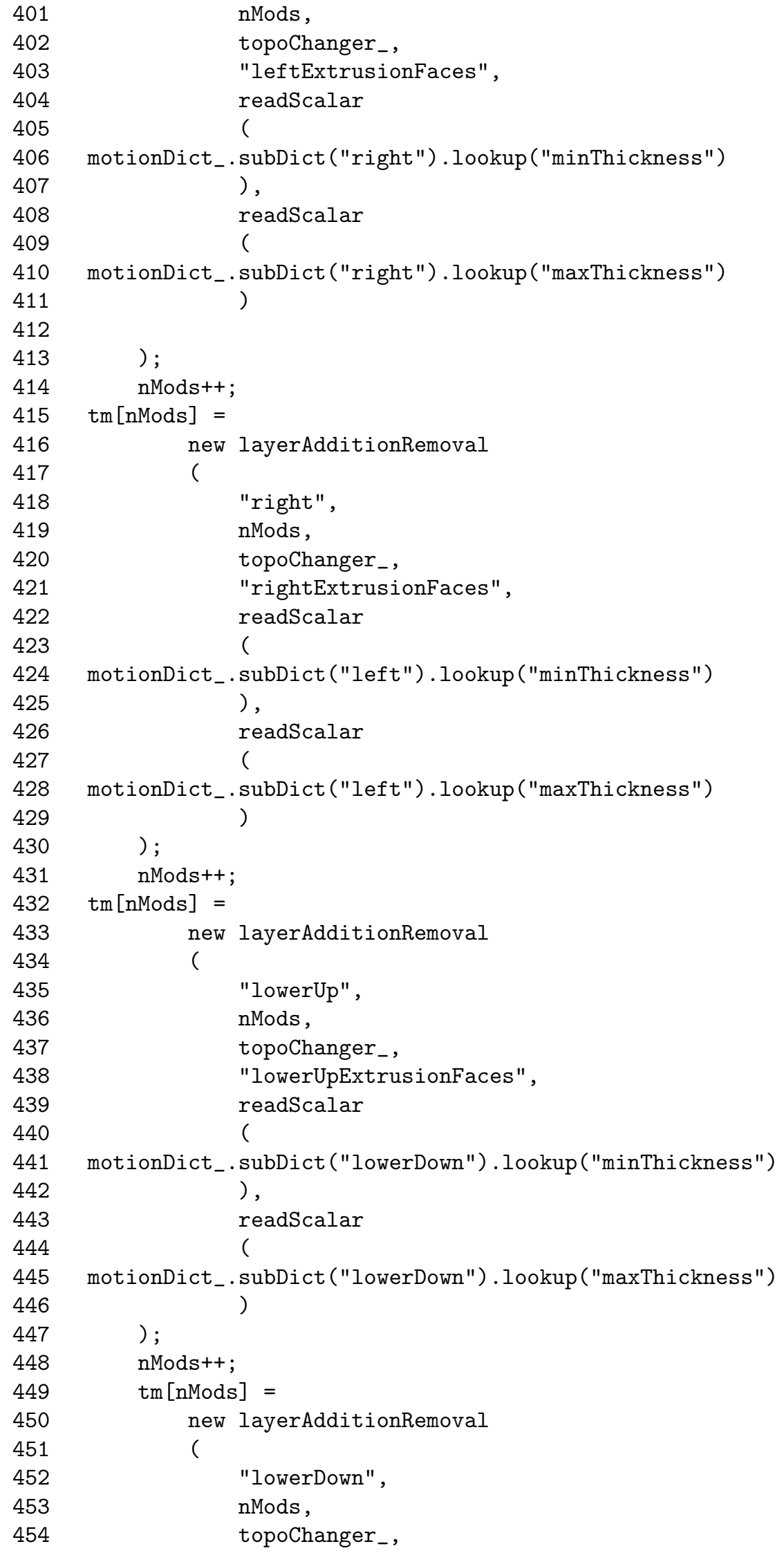




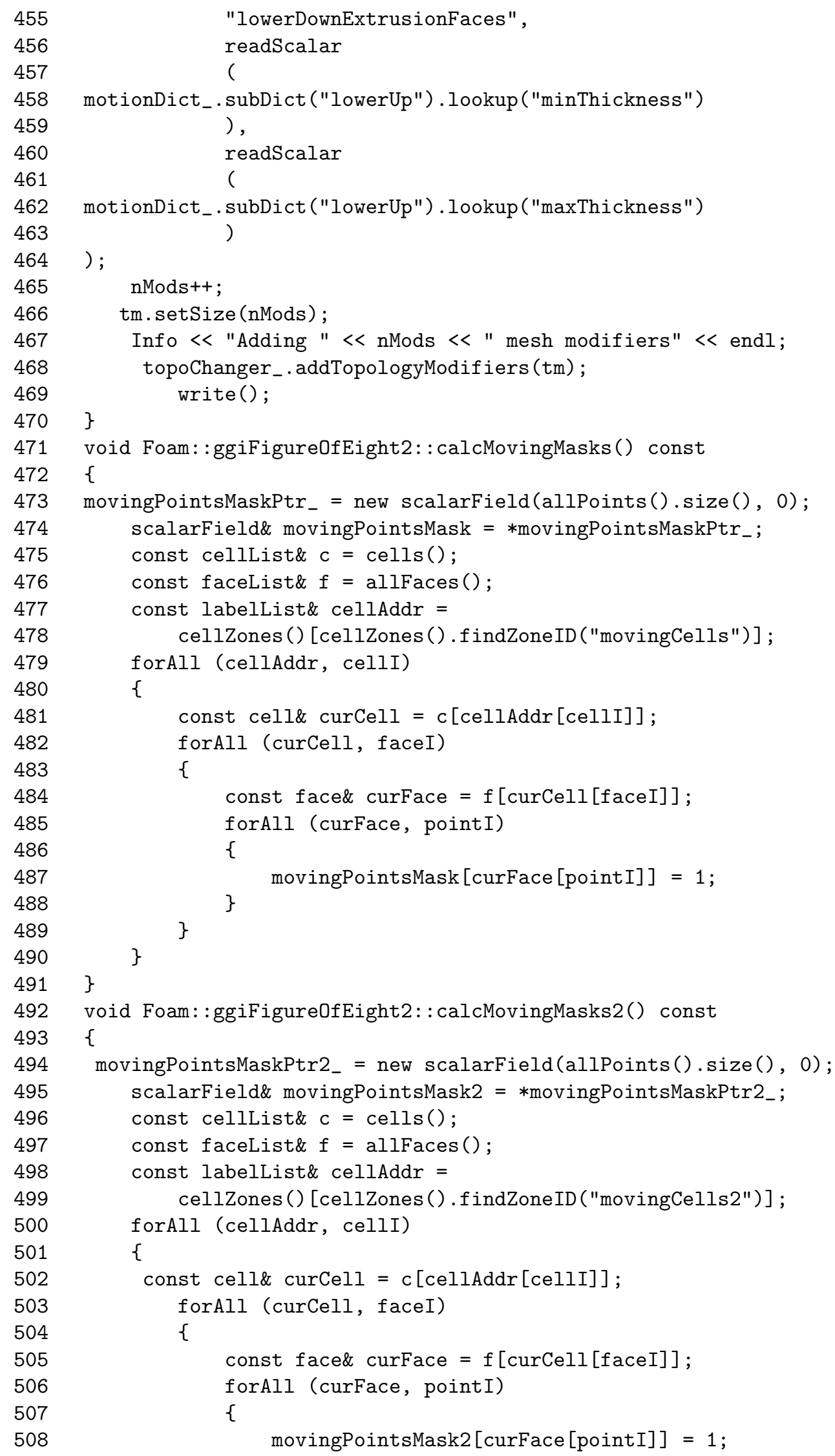

491 


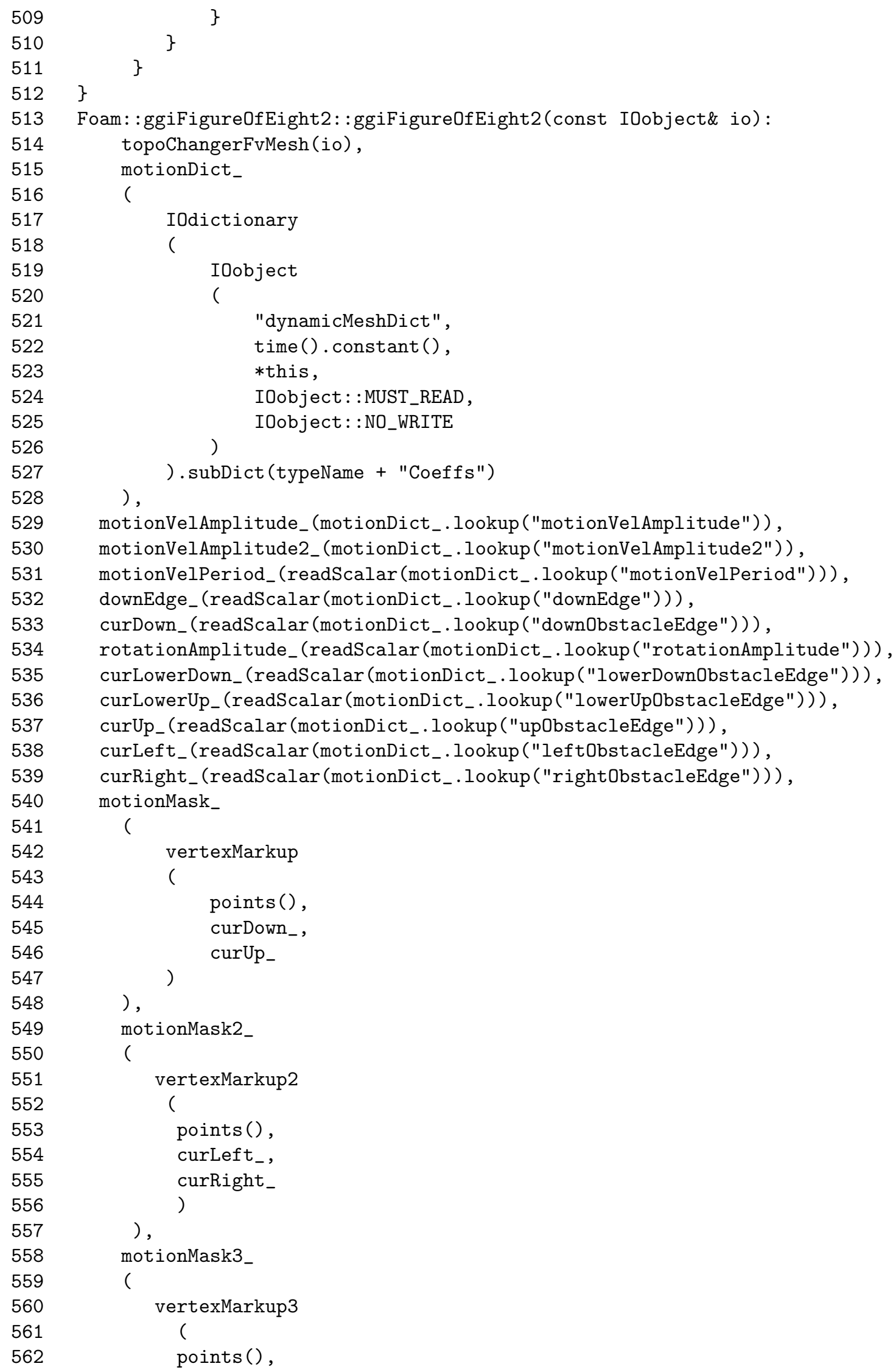




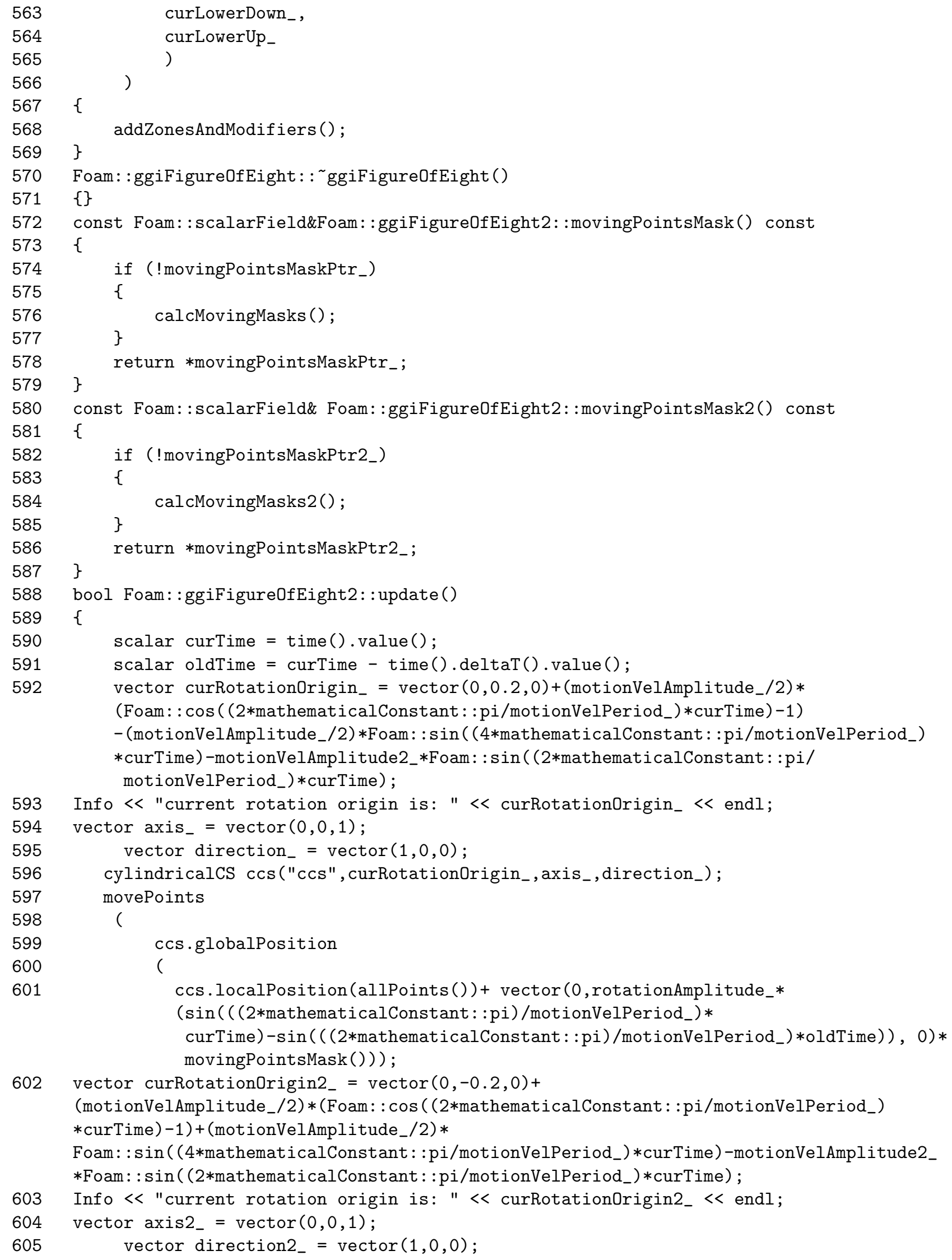




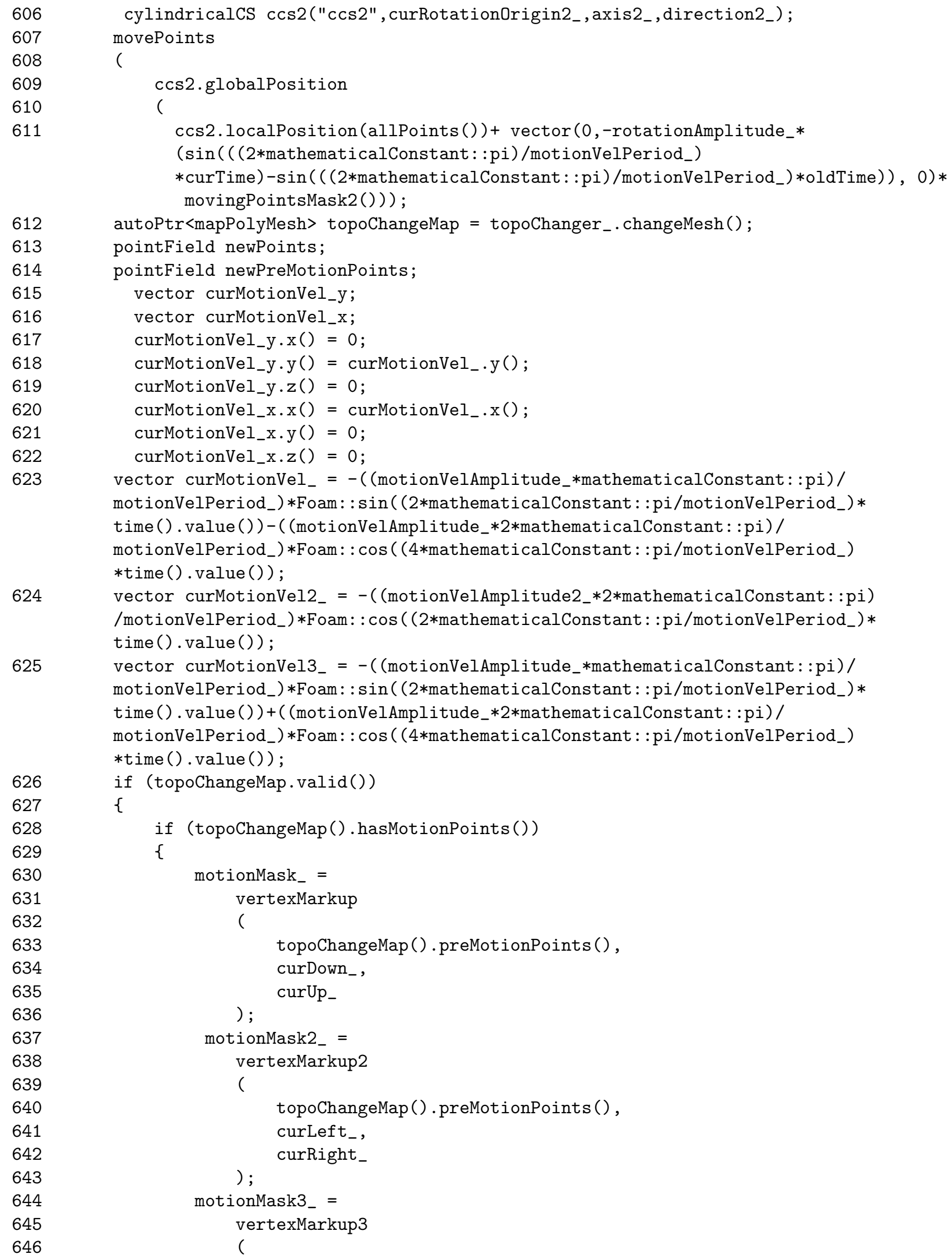




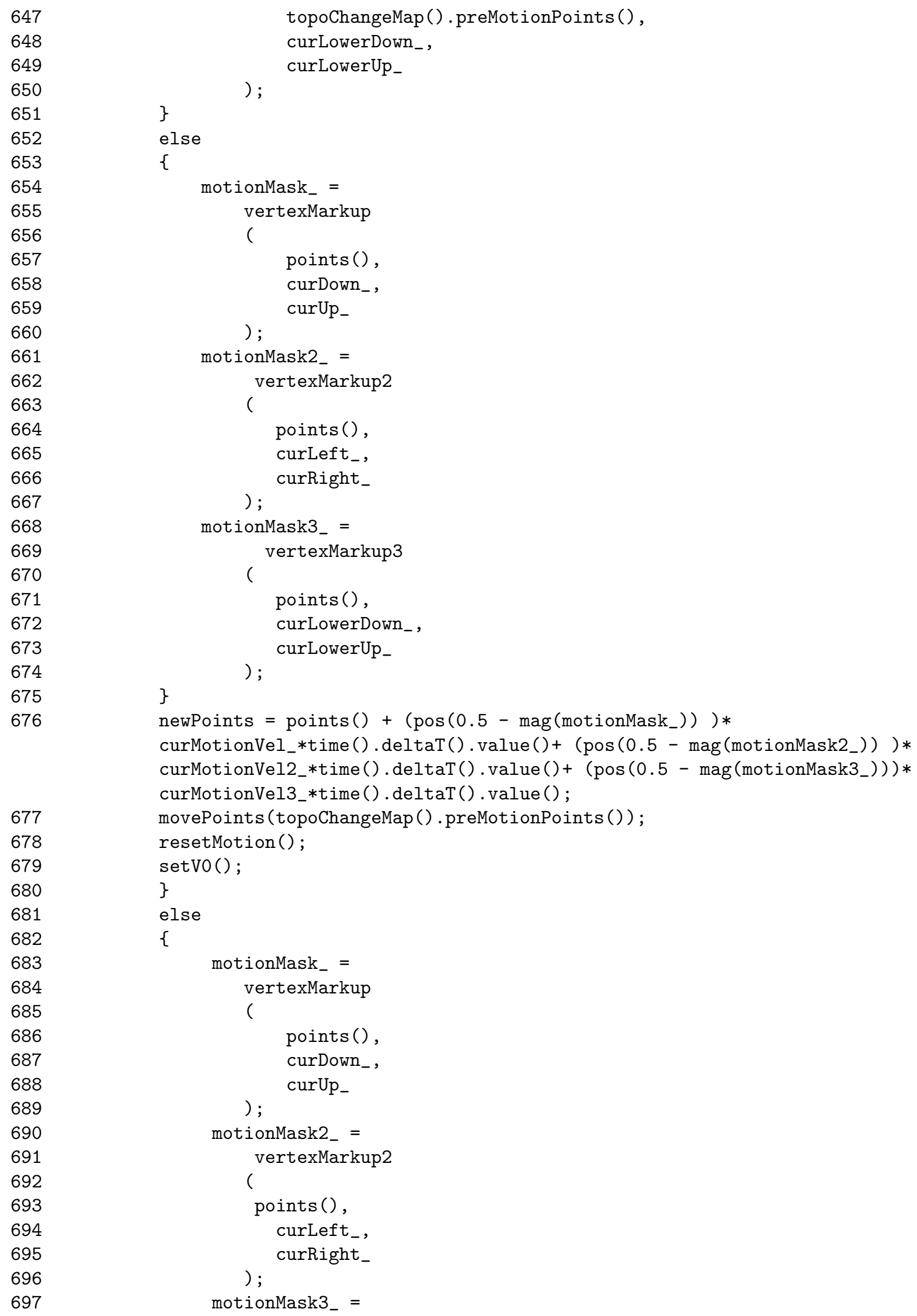




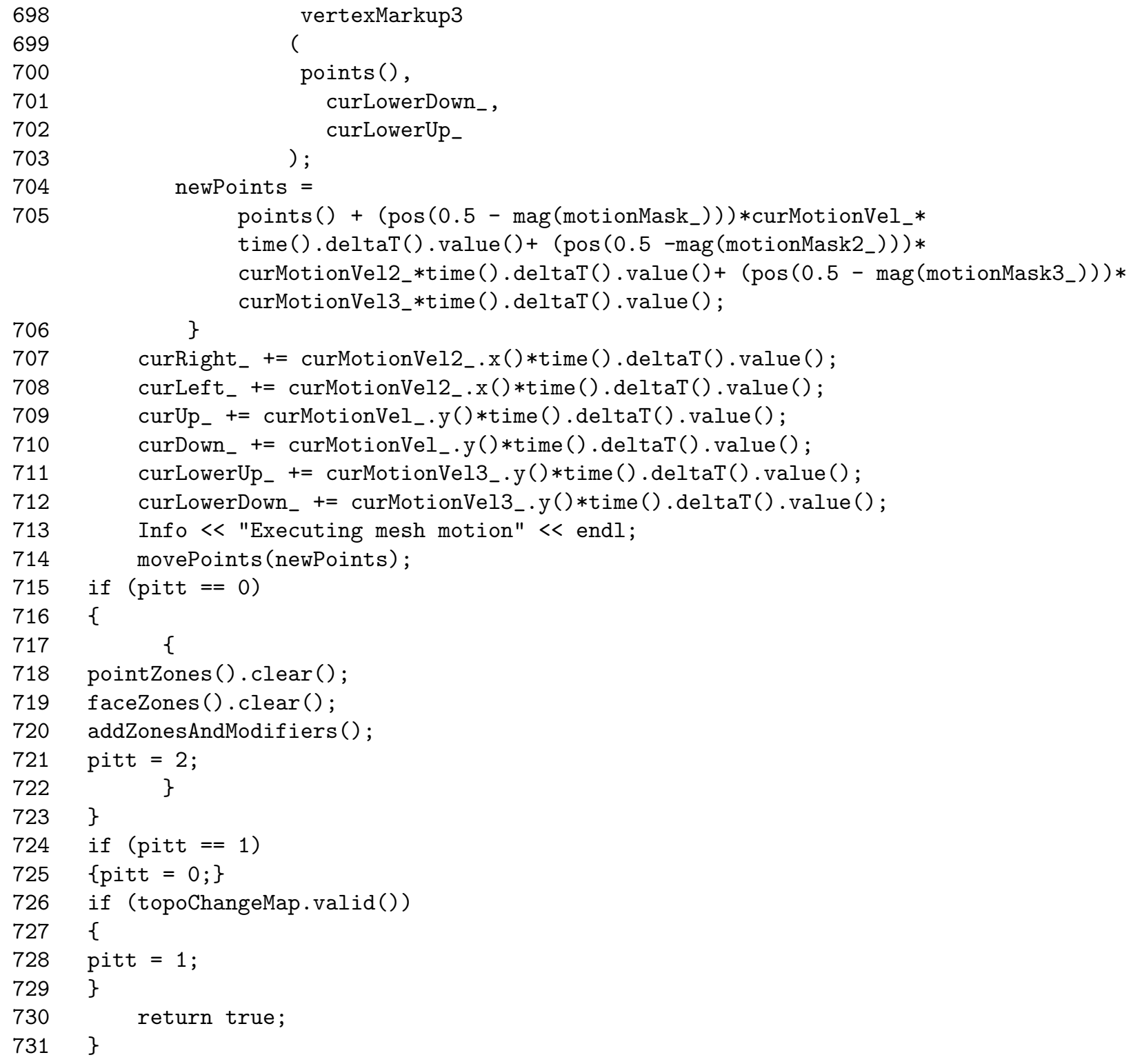

The presented ggiFigureOfEight.C file needs a header file, calling the required subroutines and classes. This file is called ggiFigureOfEight.H and presented in the following.

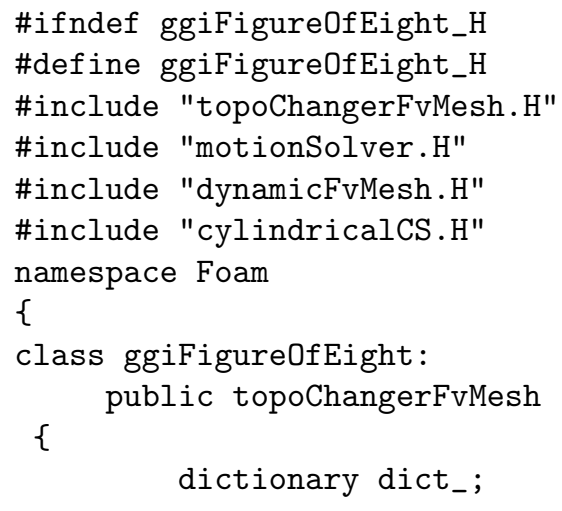




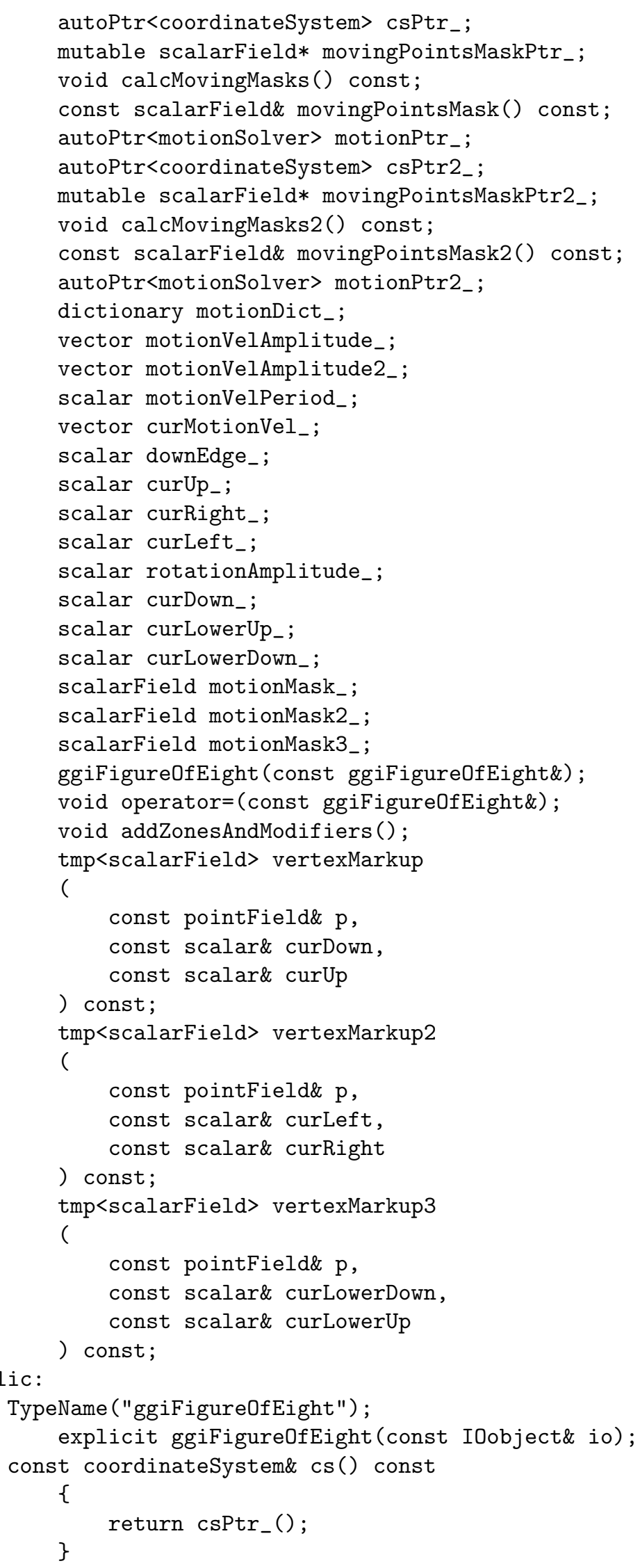




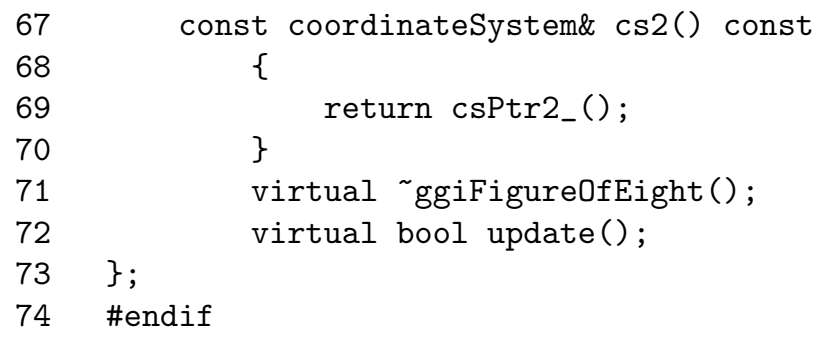




\section{Bibliography}

[1] M. H. Dickinson and K. G. Götz, "Unsteady Performance of model wings at low Reynolds numbers," Journal of Experimental Biology, vol. 174, pp. 45-64, 1993.

[2] C. P. Ellington, C. Van Den Berg, A. P. Willmott, and A. L. R. Thomas, "Leading edge vortices in insect flight," Nature (London), vol. 384, pp. 626-630, 1996.

[3] M. H. Dickinson, F. O. Lehmann, and S. P. Sane, "Wing rotation and the aerodynamic basis of insect flight," Science, vol. 284, no. 5422, pp. 1954-1960, 1999.

[4] T. Weis-Fogh, "Quick estimates of flight fitness in hovering animals, including novel mechanisms for lift production," Journal of Experimental Biology, vol. 59, pp. 169-230, 1973.

[5] A. Ania, Flapping wing devices for Mars exploration: preliminary results from a novel flapping wing mechanism. MSc thesis, Department of Mechanical Engineering, Royal Military College, 2008.

[6] M. R. Amiralaei, H. Alighanbari, and S. M. Hashemi, "Flow field characteristics study of a flapping airfoil using computational fluid dynamics," Journal of Fluids and Structures, Vol. 27, no. 7, pp. 1068-1085, 2011.

[7] C. P. Ellington, "The aerodynamics of hovering insect flight. I. The quasi-steady analysis," Philosophical Transactions of the Royal Society of London Series B, vol. 305, no. 1122, pp. 1-15, 1984. 
[8] J. G. Leishman, Principles of Helicopter Aerodynamics. Cambridge University Press, 2000.

[9] S. Heathcote and I. Gursul, "Jet switching phenomenon for a periodically plunging airfoil," Physics of Fluids, vol. 19, 027104, 2007.

[10] M. R. Amiralaei, H. Alighanbari, and S. M. Hashemi, "An investigation into the effects of unsteady parameters on the aerodynamics of a low Reynolds number pitching airfoil," Journal of Fluids and Structures, vol. 26, no. 6, pp. 979-993, 2010.

[11] M. F. M. Osborne, "Aerodynamics of flapping flight with application to insects," Journal of Experimental Biology, vol. 28, pp. 221-245, 1951.

[12] F. O. Lehmann, "The mechanisms of lift enhancement in insect flight," Naturwissenschaften, vol. 91, no. 3, pp. 101-122, 2004.

[13] W. J. McCroskey, "The phenomenon of dynamic stall," tech. rep., NASA technical memorandum, 1981.

[14] Z. J. Wang, "Dissecting Insect Flight," Annual Review of Fluid Mechanics, vol. 37, pp. 183-210, 2005.

[15] C. Van Den Berg and C. P. Ellington, "The three-dimensional leading-edge vortex of a 'hovering' model Hawkmoth," Philosophical Transactions of the Royal Society of London Series B, vol. 352, no. 1351, pp. 329-340, 1977.

[16] S. P. Sane and M. H. Dickinson, "The control of flight force by a flapping wing: lift and drag production," Journal of Experimental Biology, vol. 204, pp. 2607-2626, 2001.

[17] J. M. Birch and M. H. Dickinson, "Spanwise flow and the attachment of the leading-edge vortex on insect wings," Nature, vol. 412, pp. 729-733, 2001. 
[18] M. H. Dickinson and K. G. Götz, "The wake dynamics and flight forces of the fruit fly Drosophila melanogaster," Journal of Experimental Biology, vol. 199, pp. 2085-2104, 1996.

[19] S. P. Sane and M. H. Dickinson, "The aerodynamic effects of wing rotation and a revised quasi-steady model of flapping flight," Journal of Experimental Biology, vol. 205, pp. 1087-1096, 2002.

[20] M. Sun and J. Tang, "Unsteady aerodynamic force generation by a model fruit fly wing in flapping motion," Journal of Experimental Biology, vol. 205, pp. 55-70, 2002.

[21] J. Wu and M. Sun, "The influence of the wake of a flapping wing on the production of aerodynamic forces," Acta Mechanica Sinica, vol. 21, no. 5, pp. 411-418, 2005.

[22] J. M. Birch and M. H. Dickinson, "The influence of wingwake interactions on the production of aerodynamic forces in flapping flight," Journal of Experimental Biology, vol. 206, pp. 2257-2272, 2003.

[23] T. Maxworthy, "Experiments on the Weis-Fogh mechanism of lift generation by insects in hovering flight. Part 1. Dynamics of the fling," Journal of Fluid Mechanics, vol. 93, pp. 47-63, 1979.

[24] F. O. Lehmann, S. P. Sane, and M. H. Dickinson, "The aerodynamic effects of wingwing interaction in flapping insect wings," Journal of Experimental Biology, vol. 208, pp. 30753092, 2005.

[25] M. J. Lighthill, Mathematical biofluiddynamics. Philadelphia, PA: Society for Industry and Applied Mathematics, 1975.

[26] J. M. V. Rayner, "A vortex theory of animal flight. Part 2. The forward flight of birds," Journal of Fluid Mechanics, vol. 91, pp. 731-763, 1979.

[27] J. D. Anderson, Fundamentals of Aerodynamics. McGraw-Hill, 1991. 
[28] C. P. Ellington, "The novel aerodynamics of insect flight: applications to micro-air vehicles," Journal of Experimental Biology, vol. 202, pp. 3439-3448, 1999.

[29] F. O. Lehmann and M. H. Dickinson, "The control of wing kinematics and flight forces in fruit flies (Drosophila spp.)," Journal of Experimental Biology, vol. 201, pp. 385-401, 1998.

[30] J. Katz, Low-speed aerodynamics, from wing theory to panel methods. McGraw-Hill, 1991.

[31] N. S. Vest and J, Katz, "Unsteady aerodynamic model of flapping wings," AIAA Journal, vol. 34, no. 7, pp. 1435-1440, 1996.

[32] M. J. Smith, "Simulating moth wing aerodynamics : towards the development of flapping-wing technology," AIAA Journal, vol. 34, no. 7, pp. 1348-1355, 1996.

[33] H. Liu and K. Kawachi, "A numerical study of insect flight," Journal of Computational Physics, vol. 146, no. 1, pp. 124-156, 1998.

[34] T. Theodorsen, "General theory of aerodynamic instability and the mechanism of flutter," NACA tech. rep. 496, 1935.

[35] T. Von Karman, "Airfoil theory for non-uniform motion," Journal of the Aeronautical Sciences, vol. 5, pp. 379-390, 1938.

[36] J. G. Leishman, "Challenges in modelling the unsteady aerodynamics of wind turbines," Wind Energy, vol. 5, no. 2-3, pp. 85-132, 2002.

[37] I. E. Garrick, "On some reciprocal relations in the theory of nonstationary flows," NACA tech. rep. 629, 1938.

[38] E. Hoo, K. D. Do, and J. Pan, "An investigation on the lift force of a wing pitching in dynamic stall for a comfort control vessel," Journal of Fluids and Structures, vol. 21, no. 8, pp. 707-730, 2005. 
[39] Y. W. Jung and S. O. Park, "Vortex-shedding characteristics in the wake of an oscillating airfoil at low Reynolds number," Journal of Fluids and Structures, vol. 20, no. 3, pp. 451464, 2005.

[40] M. Fuchiwaki and K. Tanaka, "Vortex structure and scale on an unsteady airfoil," JSME International Journal Series B, vol. 49, no. 4, pp. 1056-1063, 2006.

[41] M. Fuchiwaki, K. Tanaka, H. Tanaka, and K. Kamemoto, "Flow patterns behind pitching airfoil and unsteady fluid forces," Proceedings of 3rd ASME/JSME Joint Fluids Engineering Conference, San Francisco, California, USA, 1999.

[42] M. M. Koochesfahani, "Vortical patterns in the wake of an oscillating airfoil," AIAA Journal, vol. 27, no. 9, pp. 1200-1205, 1989.

[43] C. Shih, L. M. Lourenco, and A. Krothapalli, "Investigation of flow at leading and trailing edges of pitching-up airfoil," AIAA Journal, vol. 33, no. 8, pp. 1369-1376, 1995.

[44] R. E. D. Bishop and A. Y. Hassan, "The lift and drag forces on a circular cylinder oscillating in a flowing fluid," Proceedings of the Royal Society Series A, vol. 277, no. 1368, pp. 51-75, 1964.

[45] C. H. K. Williamson and A. Roshko, "Vortex formation in the wake of an oscillating cylinder," Journal of Fluids and Structures, vol. 2, no. 4, pp. 355-381, 1988.

[46] K. M. Lam, J. C. Hu, and P. Liu, "Vortex formation processes from an oscillating circular cylinder at high KeuleganCarpenter numbers," Physics of Fluids, vol. 22, pp. 015105 (1-14), 2010.

[47] A. H. Techet, F. S. Hover, and M. S. Triantafyllou, "Vortical patterns behind a tapered cylinder oscillating transversely to a uniform flow," Journal of Fluid Mechanics, vol. 363, pp. 79-96, 1998. 
[48] K. B. Lua, T. T. Lim, K. S. Yeo, and G. Y. Oo, "Wake-structure formation of a heaving two-dimensional elliptic airfoil," AIAA Journal, vol. 45, no. 7, pp. 1571-1583, 2007.

[49] S. H. Chen and C. M. Ho, "Near wake of an unsteady symmetric airfoil," Journal of Fluids and Structures, vol. 1, no. 2, pp. 151-164, 1987.

[50] T. Prangemeier, D. Rival, and C. Tropea, "The manipulation of trailing-edge vortices for an airfoil in plunging motion," Journal of Fluids and Structures, vol. 26, no. 2, pp. 193-204, 2010.

[51] P. Freymuth, "Propulsive vortical signature of plunging and pitching airfoils," AIAA Journal, vol. 26, no. 7, pp. 881-883, 1988.

[52] J. C. S. Lai and M. F. Platzer, "Jet characteristics of a plunging airfoil," AIAA Journal, vol. 37, no. 12, pp. 1529-1537, 1999.

[53] M. S. Triantafyllou, G. S. Triantafyllou, and R. Gopalkrishnan, "Wake mechanics for thrust generation in oscillating foils," Physics of Fluids Series A, vol. 3, no. 12, pp. 2835 (1-3), 1991.

[54] D. Viieru, J. Tang, Y. Lian, H. Liu, and W. Shyy, "Flapping and flexible wing aerodynamics of low Reynolds number flight vehicles," Proceedings of 44th AIAA Aerospace Sciences Meeting and Exhibit, AIAA-2006-503, Reno, Nevada, USA, 2006.

[55] P. Freymuth, "Thrust generation by an airfoil in hover modes," Experiments in Fluids, vol. 9, no. 1-2, pp. 17-24, 1990.

[56] H. Nagai, K. Isogai, T. Fujimoto, and T. Hayase, "Experimental and numerical study of forward flight aerodynamics of insect flapping wing," AIAA Journal, vol. 47, no. 3, pp. $730-742,2009$.

[57] K. M. Isaac, J. Rolwes, and A. Colozza, "Aerodynamics of a flapping and pitching wing using simulations and experiments," AIAA Journal, vol. 46, no. 6, pp. 1505-1515, 2008. 
[58] Z. J. Wang, J. M. Birch, and M. H. Dickinson, "Unsteady forces and flows in low Reynolds number hovering flight: two-dimensional computations vs robotic wing experiments," Journal of Experimental Biology, vol. 207, pp. 449-460, 2004.

[59] B. Singh and I. Chopra, "Insect-based hover-capable flapping wings for micro air vehicles: experiments and analysis," AIAA Journal, vol. 46, no. 9, pp. 2115-2135, 2008.

[60] M. Kaya, I. H. Tuncer, K. D. Jones, and M. F. Platzer, "Optimization of flapping motion parameters for two airfoils in a biplane configuration," Journal of Aircraft, vol. 46, no. 2, pp. 583-592, 2009.

[61] N. Mahir and D. Rockwell, "Vortex formation from a forced system of two cylinders. Part I: tandem arrangement," Journal of Fluids and Structures, vol. 10, no. 10, pp. 473-489, 1996.

[62] N. Mahir and D. Rockwell, "Vortex formation from a forced system of two cylinders. Part II: side-by-side arrangement," Journal of Fluids and Structures, vol. 10, no. 10, pp. 491-500, 1996.

[63] G. R. S. Assi, J. R. Meneghini, J. A. P. Aranha, P. W. Bearman, and E. Casaprima, "Experimental investigation of flow-induced vibration interference between two circular cylinders," Journal of Fluids and Structures, vol. 22, no. 6-7, pp. 819-827, 2006.

[64] D. Rival, G. Hass, and C. Tropea, "Recovery of energy from leading- and trailing-edge vortices in tandem-airfoil configurations," Journal of Aircraft, vol. 48, no. 1, pp. 203-211, 2011.

[65] J. Warkentin and J. Delaurier, "Experimental aerodynamic study of tandem flapping membrane wings," Journal of Aircraft, vol. 44, no. 5, pp. 1653-1661, 2007. 
[66] A. Jameson, "Time dependent calculations using multigrid, with applications to unsteady flows past airfoils and wings," Proceedings of 10th AIAA Computational Fluid Dynamics Conference, AIAA-1991-1596, Honolulu, Hawaii, USA, 1991.

[67] S. Yang, S. Luo, and F. Tsai, "Subsonic flow over unstalled pitching airfoil computed by Euler method," Proceedings of 36th AIAA Fluid Dynamics Conference and Exhibit, AIAA-2006-3914, San Francisco, California, USA, 2006.

[68] M. H. Akbari and S. J. Price, "Simulation of dynamic stall for a NACA 0012 airfoil using a vortex method," Journal of Fluids and Structures, vol. 17, no. 6, pp. 855-874 , 2003.

[69] N. Okong'o and D. D. Knight, "Implicit unstructured Navier-Stokes simulation of leading edge separation over a pitching airfoil," Applied Numerical Mathematics, vol. 27, no. 3, pp. 269-308, 1998.

[70] H. Hamdani and M. Sun, "Aerodynamic forces and flow structures of an airfoil in some unsteady motions at small Reynolds number," Acta Mechanica, vol. 145, no. 1-4, pp. 173-187, 2000.

[71] J. Young and J. C. S. Lai, "Oscillation frequency and amplitude effects on the wake of a plunging airfoil," AIAA Journal, vol. 42, no. 10, pp. 2042-2052, 2004.

[72] M. R. Visbal and J. S. Shang, "Investigation of the flow structure around a rapidly pitching airfoil ," AIAA Journal, vol. 27, no. 8, pp. 1044-1051, 1989.

[73] J. S. Leontini, B. E. Stewart, M. C. Thompson, and K. Hourigan, "Wake state and energy transitions of an oscillating cylinder at low Reynolds number," Physics of Fluids, vol. 18, pp. 067101 (1-9), 2006. 
[74] O. A. Marzouk, "Direct Numerical Simulations of the Flow Past a Cylinder Moving With Sinusoidal and Nonsinusoidal Profiles," Journal of Fluids Engineering, vol. 131, no. 12, pp. 121201 (1-9), 2009.

[75] X. Yang and Z. C. Zheng, "Nonlinear spacing and frequency effects of an oscillating cylinder in the wake of a stationary cylinder," Physics of Fluids, vol. 22, pp. 043601 (1-15), 2010.

[76] J. Y. Andro and L. Jacquin, "Frequency effects on the aerodynamic mechanisms of a heaving airfoil in a forward flight configuration," Aerospace Science and Technology, vol. 13, no. 1, pp. 71-80, 2009.

[77] S. An, J. Maeng, and C. Han, "Thickness effect on the thrust generation of heaving elliptic airfoils," Journal of Aircraft, vol. 46, no. 1, pp. 216-222, 2009.

[78] S. An, J. Maeng, and C. Han, "Unsteady CFD of a plunging airfoil in low-Reynoldsnumber flow regime," Proceedings of AIAA 40th Fluid Dynamics Conference and Exhibit, AIAA-2010-4460, Chicago, Illinois, USA, 2010.

[79] D. D. J. Chandar and M. Damodaran, "Computational study of unsteady low-Reynolds number airfoil aerodynamics using moving overlapping meshes," AIAA Journal, vol. 46, no. 2, pp. 429-438, 2008.

[80] D. Lentink and M. Gerritsma, "Influence of airfoil shape on performance in insect flight," Proceedings of 33rd AIAA Fluid Dynamics Conference and Exhibit, AIAA-20033447, Orlando, Florida, USA, 2003.

[81] G. C. Lewin and H. Haj-Hariri, "Modelling thrust generation of a two-dimensional heaving airfoil in a viscous flow," Journal of Fluid Mechanics, vol. 492, pp. 339-362, 2003. 
[82] J. Pederzani and H. Haj-Hariri, "Numerical analysis of heaving flexible airfoils in a viscous flow," AIAA Journal, vol. 44, no. 11, pp. 2773-2779, 2006.

[83] J. Young and J. C. S. Lai, "Vortex lock-in phenomenon in the wake of a plunging airfoil," AIAA Journal, vol. 45, no. 2, pp. 485-490, 2007.

[84] J. S. Lee, C. Kim, and K. H. Kim, "Design of flapping airfoil for optimal aerodynamic performance in low-Reynolds number flows," AIAA Journal, vol. 44, no. 9, pp. 1960-1972, 2006.

[85] R. Mittal, Y. Uttarkar, and H. S. Udaykumar, "Computational modeling and analysis of biomimetic flight mechanisms," Proceedings of 40th AIAA Aerospace Sciences Meeting and Exposition, AIAA-2002-865, Reno, NV, USA, 2002.

[86] J. S. Lee, J. H. Kim, and C. Kim, "Numerical Study on the Unsteady-Force-Generation Mechanism of Insect Flapping Motion," AIAA Journal, vol. 46, no. 7, pp. 1835-1848, 2008.

[87] J. Tang, D. Viieru, and W. Shyy, "Effects of Reynolds number and flapping kinematics on hovering aerodynamics," AIAA Journal, vol. 46, no. 4, pp. 967-976, 2008.

[88] F. M. Bos, D. Lentink, B. W. Van Oudheusden, and H. Bijl, "Influence of wing kinematics on aerodynamic performance in hovering insect flight," Journal of Fluid Mechanics, vol. 594, pp. 341-368, 2008.

[89] T. Kinsey and G. Dumas, "Parametric study of an oscillating airfoil in a powerextraction regime," AIAA Journal, vol. 46, no. 6, pp. 1318-1330, 2008.

[90] M. Kaya and I. H. Tuncer, "Nonsinusoidal path optimization of a flapping airfoil," AIAA Journal, vol. 45, no. 8, pp. 2075-2082, 2007.

[91] M. Sun and J. Tang, "Lift and power requirements of hovering flight in Drosophila virilis," Journal of Experimental Biology, vol. 205, pp. 2413-2427, 2002. 
[92] J. Young, J. C. S. Lai, and C. Germain, "Simulation and parameter variation of flappingwing motion based on dragonfly hovering," AIAA Journal, vol. 46, no. 4, pp. 918-924, 2008.

[93] H. Dong, R. Mittal, M. Bozkurttas, and F. Najjar, "Wake structure and performance of finite aspect-ratio flapping foils," Proceedings of 43rd AIAA Aerospace Sciences Meeting and Exposition, AIAA-2005-81, Reno, NV, USA, 2005.

[94] R. Ramamurti and W. C. Sandberg, "A three-dimensional computational study of the aerodynamic mechanisms of insect flight," Journal of Experimental Biology, vol. 205, pp. $1507-1518,2002$.

[95] R. Ramamurti and W. Sandberg, "Computations of insect and fish locomotion with applications to unconventional unmanned vehicles," AIAA Journal, vol. 46, no. 9, pp. 2178-2190, 2008.

[96] M. J. Chern, P. R. Kanna, Y. J. Lu, I. C. Cheng, and S. C. Chang, "A CFD study of the interaction of oscillatory flows with a pair of side-by-side cylinders," Journal of Fluids and Structures, vol. 26, no. 4, pp. 626-643, 2010.

[97] D. S. Lee, M. Y. Ha, H. S. Yoon, and S. Balachandar, "A numerical study on the flow patterns of two oscillating cylinders," Journal of Fluids and Structures, vol. 25, no. 2, pp. 263-283, 2009.

[98] S. Mittal and V. Kumar, "Flow-induced oscillations of two cylinders in tandem and staggered arrangements," Journal of Fluids and Structures, vol. 15, no. 5, pp. 717-737, 2001.

[99] G. V. Papaioannou, D. K. P. Yue, M. S. Triantafyllou, and G. E. Karniadakis, "On the effect of spacing on the vortex-induced vibrations of two tandem cylinders," Journal of Fluids and Structures, vol. 24, no. 6, pp. 833-854, 2008. 
[100] T. K. Prasanth and S. Mittal, "Vortex-induced vibration of two circular cylinders at low Reynolds number," Journal of Fluids and Structures, vol. 25, no. 4, pp. 731-741, 2009.

[101] S. Singha and K. P. Sinhamahapatra, "High-resolution numerical simulation of low Reynolds number incompressible flow about two cylinders in tandem," Journal of Fluids Engineering, vol. 132, pp. 011101 (1-10), 2010.

[102] W. Jester and Y. Kallinderis, "Numerical study of incompressible flow about transversely oscillating cylinder pairs," Journal of Offshore Mechanics and Arctic Engineering, vol. 126 , no. 4, pp. 310 (1-8), 2004.

[103] I. Akhtar, R. Mittal, G. V. Lauder, and E. Drucker, "Hydrodynamics of a biologically inspired tandem flapping foil configuration," Theoretical and Computational Fluid Dynamics, vol. 21, no. 3, pp. 155-170, 2007.

[104] J. H. Ferziger and M. Peric, Computational Methods for Fluid Dynamics. SpringerVerlag, 2002.

[105] H. K. Versteeg and W. Malalasekera, An Introduction to Computational Fluid Dynamics: The Finite Volume Method. Pearson Prentice-Hall, 2007.

[106] H. Schlichting, Boundary Layer Theory. McGraw-Hill, 2000.

[107] H. Jasak and Z. Tuković, "Automatic mesh motion for the unstructured finite volume method," Transactions of FAMENA, vol. 30, pp. 1-18, 2007.

[108] S. V. Patankar and D. B. Spalding, "A calculation procedure for heat, mass and momentum transfer in three-dimensional parabolic flows," International Journal of Heat and Mass Transfer, vol. 15, no. 10, pp. 1787-1806, 1972. 
[109] R. I. Issa, "Solution of the implicitly discretised fluid flow equations by operatorsplitting," International Journal of Heat and Mass Transfer, vol. 62, no. 1, pp. 40-65 , 1986.

[110] F. H. Harlow and J. E. Welch, "Numerical computation of time dependent viscous flows with free surface," Physics of Fluids, vol. 8, no. 12, pp. 2182-2189, 1965.

[111] C. M. Rhie and W. L. Chow, "Numerical study of the turbulent flow past an airfoil with trailing edge separation ," AIAA Journal, vol. 21, no. 11, pp. 1525-1532, 1983.

[112] H. Jasak, Error analysis and estimation in the finite volume method with applications to fluid flow. PhD thesis, Imperial College, University of London, 1996.

[113] M. C. Lai and C. S. Peskin, "An immersed boundary method with formal second-order accuracy and reduced numerical viscosity," Journal of Computational Physics, vol. 160, no. 2, pp. 705-719, 2000.

[114] F. M. Bos, Numerical simulations of flapping foil and wing aerodynamics: Mesh deformation using radial basis functions. PhD thesis, Delft University of Technology, 2010.

[115] G. Chesshire and W. D. Henshaw, "Composite overlapping meshes for the solution of partial differential equations," Journal of Computational Physics, vol. 90, no. 1, pp. 1-64, 1990.

[116] J. T. Batina, "Unsteady Euler airfoil solutions using unstructured dynamic meshes," AIAA Journal, vol. 28, no. 8, pp. 1381-1388, 1990.

[117] F. J. Blom, "Considerations on the spring analogy," International Journal for Numerical Methods in Fluids, vol. 32, no. 6, pp. 647668, 2000.

[118] C. Farhat, C. Degand, B. Koobus, and M. Lesoinne, "Torsional springs for twodimensional dynamic unstructured fluid meshes," Computer Methods in Applied Mechanics and Engineering, vol. 163, no. 1-4, pp. 231-245 , 1998. 
[119] C. Degand and C. Farhat, "A three-dimensional torsional spring analogy method for unstructured dynamic meshes," Computers and Structures, vol. 80, no. 3-4, pp. 305-316, 2002.

[120] H. Baruh, Analytical Dynamics. McGraw-Hill, 1999.

[121] D. L. Littlefield, "The use of r-adaptivity with local, intermittent remesh for modeling hypervelocity impact and penetration," International Journal of Impact Engineering, vol. 26, no. 1-10, pp. 433-442, 2001.

[122] R. Löhner and C. Yang, "Improved ALE mesh velocities for moving bodies," Communications in Numerical Methods in Engineering, vol. 12, no. 10, pp. 599-608, 1996.

[123] B. T. Helenbrook, "Mesh deformation using the biharmonic operator," International Journal for Numerical Methods in Engineering, vol. 56, no. 7, pp. 1007-1021, 2003.

[124] A. A. Johnson and T. E. Tezduyar, "Mesh update strategies in parallel finite element computations of flow problems with moving boundaries and interfaces," Computer Methods in Applied Mechanics and Engineering, vol. 119, no. 1-2, pp. 73-94, 1994.

[125] G. Chiandussi, G. Bugeda, and E. Oñate, "A simple method for automatic update of finite element meshes," Communications in Numerical Methods in Engineering, vol. 16, no. 1, pp. 1-19, 2000.

[126] R. P. Dwight, "Robust mesh deformation using the linear elasticity equations," in Computational Fluid Dynamics 2006, pp. 401-406, 2006.

[127] Z. J. Wang and A. J. Przekwas, "Unsteady flow computation using moving grid with mesh enrichment," AIAA 32nd Aerospace Sciences Meeting and Exhibit, AIAA-1994-285, Reno, NV, USA, 1994. 
[128] A. de Boer, M. S. van der Schoot, and H. Bijl, "Mesh deformation based on radial basis function interpolation," Computers and Structures, vol. 85, no. 11-14, pp. 784-795, 2007.

[129] M. A. Potsdam and G. P. Guruswamy, "A parallel multiblock mesh movement scheme for complex aeroelastic applications," AIAA 39th Aerospace Sciences Meeting and Exhibit, AIAA-2001-0716, Reno, NV, USA, 2001.

[130] S. Jakobsson and O. Amoignon, "Mesh deformation using radial basis functions for gradient-based aerodynamic shape optimization," Computers and Fluids, vol. 36, no. 6, pp. 1119-1136, 2007.

[131] H. Jasak and Z. Tuković, "Dynamic mesh handling in OpenFOAM applied to fluidstructure integration simulations," European Conference on Computational Fluid Dynamics, ECCOMAS CFD, Lisbon, Portugal, 2010.

[132] M. Beaudoin and H. Jasak, "Development of an arbitrary mesh interface for turbomachinery simulations with OpenFOAM," Open Source CFD International Conference, Berlin, Germany, 2008.

[133] F. M. Mahfouz and H. M. Badr, "Flow structure in the wake of a rotationally oscillating cylinder," Journal of Fluids Engineering, vol. 122, no. , pp. 290-301, 2000.

[134] O. Okajima, H. Takata, and T. Asanuma, "Viscous Flow around a rotationally Oscillating Circular Cylinder," Inst. Space Aero. Sci. rep. 532, 1975.

[135] M. R. Amiralaei, H. Alighanbari, and S. M. Hashemi, "Unsteady CFD of a plunging airfoil in low Reynolds number flow regime," Proceedings of 2010 AIAA 40th Fluid Dynamics Conference and Exhibit, Chicago, Illinois, USA, 2010. 
[136] C. H. K. Williamson, "Vortex dynamics in the wake of a cylinder in fluid vortices," in Fluid Mechanics and its Applications (ed. S. I. Green), chap 5, Kluwer Academic Publishing, Dordrecht.

[137] E. Guilmineau and P. Queutey, "A numerical simulation of vortex shedding from an oscillating circular cylinder," Journal of Fluids and Structures, vol. 16, no. 6, pp. 773-794, 2002.

[138] Y. Lian and W. Shyy, "Aerodynamics of low Reynolds number plunging airfoil under gusty environment," Proceedings of 45th AIAA Aerospace Sciences Meeting and Exhibit, Reno, Nevada, USA, 2007.

[139] J. Young, Numerical simulation of the unsteady aerodynamics of flapping airfoils. PhD thesis, University of New South Wales, 2005.

[140] Z. J. Wang, "The role of drag in insect hovering," Journal of Experimental Biology, vol. 207, pp. 4147-4155, 2004.

[141] H. Jasak, "Dynamic mesh handling in OpenFOAM," Proceedings of 2009 47th AIAA Aerospace Sciences and Meeting, Orlando, Florida, USA, 2009. 